\title{
Anti-Neutrino Charged Current Quasi-Elastic Scattering in $\operatorname{MINER} \nu \mathrm{A}$
}

\author{
by \\ Jesse Chvojka \\ Submitted in Partial Fulfillment \\ of the \\ Requirements for the Degree of \\ Doctor of Philosophy \\ Supervised by \\ Professor Kevin McFarland \\ Department of Physics and Astronomy \\ Arts, Sciences and Engineering \\ University of Rochester \\ Rochester, New York
}


Dedicated to My Mother 


\section{Biographical Sketch}

The author He recieved his Bachelor of Science degree in physics from Michigan State University (MSU) in 2003. The student initially studied botany and ecology and performed biological research at MSU as an undergraduate before later studying physics. He performed research at the National Superconducting Cyclotron Laboratory, located on the MSU campus. This activity involved assisting in the construction of the Focal Plane Detector for the S800 high-resolution spectrometer. He was involved in top physics analysis as an undergraduate with Kirsten Tollefson. He also performed research at Fermi National Accelerator Laboratory as a Researach Experience for Undergradudates student under the advisement of Paul Tipton formerly of the University of Rochester.

He has attended the University of Rochester since 2003. He has been involved in student outreach, involving a program to involve high school students with high energy physics. This included a collaborative project with high school students to field a cosmic ray experiment on an airplane to verify relativistic time dilation in cosmic ray muons. He received a Master of Arts in physics from the University of Rochester in 2005. He has since assisted in research and development as well as construction of the MINER $\nu$ A detector. He has been involved with a project to understand backgrounds in muon monitors located in the NuMI beamline. He

has also assisted in development of MINER $\nu \mathrm{A}$ reconstruction as well as one of the earliest physics analyses in MINER $\nu \mathrm{A}$. 


\section{Acknowledgments}

I owe thanks to many people for the support I have been given as a graduate student. I owe much to my family. My wife Kyeorda has been so supportive. She has shown great forbearance for my unsolicited physics lectures. My son Micah has made sure that my days are enjoyable and my sleep minimal. My sister has been a tremendous help during the last several years, gladly offering relief to her nephew's overworked parents. I owe much to my own parents, my Mom, Dad, and stepmother Carol who have supported me throughout my life. My Dad's lectures on science and mechanics helped inspire my interest in physics and for that I will always be grateful. And my extended family helped immeasurably in the hardest of times.

I must thank all those who have helped contribute to my professional developemnt. I have a deep gratitude to my advisor Kevin, who has been a joy to work with. I have had the blessing of having great colleagues, in particular Joel Mosseau, Jaewon Park, Dave Schmitz, Gabe Perdue, Heidi Schellman, and Bob Bradford. I would also like to thank my close collaborator Laura Fields. It has been an honor to work with you.

I want to express appreciation to the very professional support staff I have worked

with including Barb Warren, Laura Blumkin, Connie Jones, Sue Brightman, and Crae Tate. 


\section{Abstract}

The phenomenon of neutrino oscillation is becoming increasingly understood with results from accelerator-based and reactor-based experiments, but unanswered questions remain. The proper ordering of the neutrino mass eigenstates that compose the neutrino flavor eigenstates is not completely known. We have yet to detect $\mathrm{CP}$ violation in neutrino mixing, which if present could help explain the asymmetry between matter and anti-matter in the universe. We also have not resolved whether sterile neutrinos, which do not interact in any Standard Model interaction, exist. Accelerator-based experiments appear to be the most promising candidates for resolving these questions; however, the ability of present and future experiments to provide answers is likely to be limited by systematic errors. A significant source of this systematic error comes from limitations in our knowledge of neutrino-nucleus interactions. Errors on cross-sections for such interactions are large, existing data is sometimes contradictory, and knowledge of nuclear effects is incomplete. One type of neutrino interaction of particular interest is charged current quasi-elastic (CCQE) scattering, which yields a final state consisting of a charged lepton and nucleon. This process, which is the dominant interaction near energies of $1 \mathrm{GeV}$, is of great utility to neutrino oscillation experiments since the incoming neutrino energy and the square of the momentum transferred to the final state nucleon, $\mathrm{Q}^{2}$, can be reconstructed using the final state lepton kinematics. To address the uncertainty in our knowledge of neutrino interactions, many experiments have begun making dedicated measurements. In particular, the MINER $\nu \mathrm{A}$ experiment is studying neutrino-nucleus interactions in the few $\mathrm{GeV}$ region. MINER $\nu \mathrm{A}$ is a fine-grained, high precision, high statistics neutrino scattering experiment that will greatly im- 
prove our understanding of neutrino cross-sections and nuclear effects that affect the final state particles in neutrino interactions. We present the first cross-section measurement for MINER $\nu \mathrm{A}$, the differential cross-section $\frac{\mathrm{d} \sigma}{\mathrm{dQ}^{2}}$ for muon anti-neutrino CCQE scattering on polystyrene scintillator $(\mathrm{CH})$ as well as comparisons to several final state models. 


\section{Contributors and Funding Sources}

The defense committee for this thesis is composed of Steven Manly, Lynne Orr, Allan Greenleaf, advisor of the student Kevin McFarland, and a committee chairman to be appointed.

The student performed this work as a member of the MINER $\nu \mathrm{A}$ collaboration, the composition of which is given in Appendix C. The student was involved in several research and development projects for the detector including optical fiber tests and the Vertical Slice Test, assisted in online monitoring for the detector, took part in the construction of the detector, took operational shifts for data taking with the detector, and was involved with developing parts of the reconstruction used for this analysis. The student contributed to the track matching framework, the time slicing algorithm described later in the thesis, development and intergration of the ArgoNeuT simulation, as well as some final processing steps for tracking. The student did this as part of a collaborative effort that developed the reconstruction and simulation used for the research presented in this thesis. Additionally, the physics analysis was done jointly with Laura Fields, a postdoctoral fellow at Northwestern University.

Funding was provided by the Department of Energy (DOE). Specifically, DOE grants DE-FG02-91ER40685 and DE-SC000475 funded this research.

FNAL is operated by Fermi Research Alliance, LLC under Contract No. DeAC02-07CH11359 with the United States Department of Energy. 


\section{Contents}

Introduction $\quad$ xxii

1 Introduction 1

1.1 The Standard Model . . . . . . . . . . . . . . . . . . . 2

1.1.1 Elementary Particles . . . . . . . . . . . . . . . 2

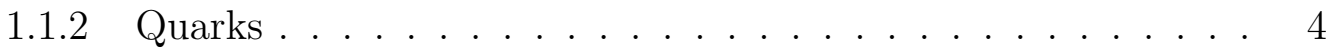

1.1.3 Leptons ..................... 5

1.1.4 Fundamental Interactions . . . . . . . . . . . . . . 7

1.1.5 Limitations of the Standard Model . . . . . . . . . . . . . . 10

1.2 Neutrino Oscillation ................... 11

1.3 Neutrino Scattering . . . . . . . . . . . . . . . . . . . . 16

1.3.1 Charged Current Quasi-Elastic Scattering . . . . . . . . 19

1.3.2 Vector Form Factors . . . . . . . . . . . . . . . . 21

1.3.3 Pseudo-Scalar Form Factor . . . . . . . . . . . . . . . . . 22

1.3.4 Conserved Vector Current Hypothesis . . . . . . . . . . . . . . 23

1.3.5 Axial Form Factor . . . . . . . . . . . . . . . . 23

1.3.6 Nuclear Effects .................... 25 
2 The Accelerator Complex 30

2.1 Accelerating Protons . . . . . . . . . . . . . . . . 30

2.2 The NuMI Beamline . . . . . . . . . . . . . . . . . . . . . . . 32

3 The MINER $\nu$ A Detector $\quad 39$

3.1 The MINER $\nu$ A ID . . . . . . . . . . . . . . . . . . 39

3.1.1 The Tracker . . . . . . . . . . . . . . . . . . . . 40

3.1 .2 The ECAL . . . . . . . . . . . . . . . 42

3.1.3 The HCAL . . . . . . . . . . . . . . . . . . . . 43

3.2 The MINER $\nu \mathrm{A}$ OD . . . . . . . . . . . . . . . . 43

3.3 Description of Frozen Detector . . . . . . . . . . . . . . 43

3.4 The Mirror Plane . . . . . . . . . . . . . . . . . . . . . . . . . . . . 44

3.5 Plane Composition .................... 45

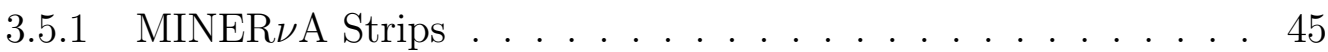

3.5.2 MINER $\nu \mathrm{A}$ WLS and Clear Optical Fiber . . . . . . . . . . . . 46

3.5.3 Material Composition of the Tracker . . . . . . . . . . . . . . 47

3.6 MINER $\nu \mathrm{A}$ PMTs . . . . . . . . . . . . . . . . . . . . 48

3.7 MINER $\nu$ A Electronics and Data Acquisition . . . . . . . . . . . . . 49

3.7.1 MINER $\nu$ A Front End Boards . . . . . . . . . . . . 50

3.7.2 Rack Mounted Electronics . . . . . . . . . . . . . . . 52

3.7.3 The Data Acquistion Computer . . . . . . . . . . . . . . 53

3.7.4 Data Taking Procedures ................ . 54

3.7.5 Special Calibration Gates . . . . . . . . . . . 55

3.7.6 Nearline Data Monitoring . . . . . . . . . . . . . 56

3.8 Descriptions of the MINOS Detector . . . . . . . . . . . . 57 
3.8 .1 MINOS Planes . . . . . . . . . . . . . . . . . . 57

3.8 .2 The MINOS Coil . . . . . . . . . . . . . . . . 58

3.9 The ArgoNeuT Detector . . . . . . . . . . . . . . . . . . . 59

4 Reconstructing and Simulating Neutrino Interactions in MINER $\nu$ A 61

4.1 Low Level Data Processing . . . . . . . . . . . . . . . . . . . . 61

4.1 .1 Pedestal Subtraction . . . . . . . . . . . . . . . . . . . . 62

4.1 .2 Applying Calibrations . . . . . . . . . . . . . . . . 62

4.1.3 Mapping Electronics Channels to Detector Channels . . . . . 75

4.1.4 Information from the NuMI Beam . . . . . . . . . . . . 76

$4.2 \quad$ MINER $\nu$ A Monte Carlo . . . . . . . . . . . . . . . . . . 77

4.2.1 Simulation of the NuMI Beam . . . . . . . . . . . . . 77

4.2 .2 Central Value Reweighting . . . . . . . . . . . . . . 78

4.2 .3 The GENIE Simulation . . . . . . . . . . . . . . . . . . . 79

4.2.4 Simulating MINER $\nu \mathrm{A} \ldots \ldots \ldots 1$

4.2.5 The ArgoNeuT Simulation . . . . . . . . . . . . . . 81

4.2 .6 The MINOS Simulation . . . . . . . . . . . . . . . . 82

4.3 High Level Data Processing . . . . . . . . . . . . . . . . . 83

4.3.1 Matching MINOS and MINER $\nu$ A Data Sets . . . . . . . . 83

4.3 .2 Forming Time Slices . . . . . . . . . . . . . . . . . . . 84

4.3 .3 Forming Clusters . . . . . . . . . . . . . . . . . 85

4.3 .4 Tracking . . . . . . . . . . . . . . . . 87

4.3.5 Attenuation Correction . . . . . . . . . . . . . . . . . . 93

4.3.6 Incorporating MINOS Reconstruction . . . . . . . . . . . . . 94

4.3.7 Muon Energy Reconstruction _. . . . . . . . . . . . . . 97 
4.4 Blob Formation . . . . . . . . . . . . . . . . . . . . . . . . . . 98

4.5 Reconstructing Neutrino Interactions _ . . . . . . . . . . . . . . . 99

\section{Measuring the Charged Current Quasi-Elastic Differential Cross-}

$\begin{array}{ll}\text { Section } \frac{\mathrm{d} \sigma}{\mathrm{d} \mathrm{Q}^{2}} & 101\end{array}$

5.1 Event Selection . . . . . . . . . . . . . . . . . . . . . 101

5.1.1 Isolating a Charged Current Sample . . . . . . . . . . . . . 102

5.1 .2 Vetoing Rock Muon Events . . . . . . . . . . . . . . . . . 103

5.1 .3 CCQE Event Selection . . . . . . . . . . . . . . . . 107

5.1.4 Defining the Signal Sample in Monte Carlo . . . . . . . . . . . 114

5.2 Calculation of the Differential CCQE Cross-Section $\frac{\mathrm{d} \sigma}{\mathrm{dQ}_{\mathrm{QE}}^{2}} \ldots \ldots . . .116$

5.2 .1 Backgrounds . . . . . . . . . . . . . . . . . . . 117

5.2.2 Unfolding Detector Smearing . . . . . . . . . . . . . . 122

5.2.3 Efficiency and Acceptance Corrections . . . . . . . . . . . 126

5.2 .4 Flux . . . . . . . . . . . . . . . . . . . . . . . . 133

5.2 .5 Number of Target Protons . . . . . . . . . . . . . . . . 133

5.3 Systematic Errors . . . . . . . . . . . . . . . . . . . . . . . 134

5.3 .1 Muon Reconstruction . . . . . . . . . . . . . . . . . . 137

5.3.2 GENIE and Final State Interaction Modeling Errors _. . . . 144

5.3.3 Geant4 Modeling Errors . . . . . . . . . . . . . . . . . . 148

5.3.4 Uncertainties on Recoil Energy, Recoil Multiplicity, and Event Selection . . . . . . . . . . . . . . . 156

5.3.5 Systematic Error on the Flux . . . . . . . . . . . . . . 161

5.3 .6 Miscellaneous . . . . . . . . . . . . . . . . . . 163

5.3.7 Total Systematic Error . . . . . . . . . . . . . . . . . . 164 
5.4 Comparing $\frac{\mathrm{d} \sigma}{\mathrm{d} Q_{\mathrm{QE}}^{2}}$ with Other Models . . . . . . . . . . . . . 169

6 Conclusions 171

$\begin{array}{ll}\text { Bibliography } & 173\end{array}$

A Reconstruction and Resolution Plots for the CCQE Analysis 183

B The Vertical Slice Test 206

B.1 The VST Aparatus . . . . . . . . . . . . . . . . . . . 206

B.1.1 Dark Box . . . . . . . . . . . . . . 206

B.1.2 VST Array . . . . . . . . . . . . . . . . . . 208

B.1.3 VST PMT . . . . . . . . . . . . . . . . . . . . . . . . 209

B.1.4 VST Trigger . . . . . . . . . . . . . . . . . . . 209

B.1.5 VST Electronics . . . . . . . . . . . . . . . . . . 210

B.1.6 VST Data Acquisition . . . . . . . . . . . . . . . . . 212

B.1.7 Scaling the VST to MINER $\nu$ A . . . . . . . . . . . . 214

B.2 Performing Tests with the VST . . . . . . . . . . . . 215

B.2.1 Running the VST . . . . . . . . . . . . . . . 215

B.2.2 Mapping the Array . . . . . . . . . . . . . . . . . 216

B.2.3 Charge to ADC Ratio and TriP Chip Gain . . . . . . . . 216

B.2.4 Pedestal Measurements . . . . . . . . . . . . . . . . . 217

B.2.5 Single Photoelectron Peak . . . . . . . . . . . . . 220

B.2.6 Low Gain ADC to High Gain ADC Ratio . . . . . . . . . 224

B.2.7 VST Tracking . . . . . . . . . . . . . . . . . . . 225

B.3 The Results of the VST . . . . . . . . . . . . . . 227

B.3.1 Light Yield . . . . . . . . . . . . . . . . . . . . 227 
B.3.2 Position Residual . . . . . . . . . . . . . . . . . . . . . . 228

B.3.3 The Effect of Reduced Light Levels on Position Resolution . . 232

B.3.4 Timing Resolution . . . . . . . . . . . . . . . . . . 233

B.3.5 Concluding Thoughts on the VST . . . . . . . . . . . 237

C List of MINER $\nu$ A Collaborators 


\section{List of Tables}

1.1 Standard Model fermions . . . . . . . . . . . . . . . . . . . 4

1.2 Standard Model bosons . . . . . . . . . . . . . . . . . . . . . . 8

1.3 Neutrino mixing parameters . . . . . . . . . . . . . . . . . . . . 14

3.1 Number of atoms per plane per $\mathrm{cm}^{2}$ for the fiducial volume . . . . . 48

3.2 Front End Board gains . . . . . . . . . . . . . . . 50

5.1 The smearing matrix that relates true $\mathrm{Q}_{\mathrm{QE}}^{2}$ to reconstructed $\mathrm{Q}_{\mathrm{QE}}^{2} \cdots 125$

5.2 Uncertainites on the smearing matrix that relates true $\mathrm{Q}_{\mathrm{QE}}^{2}$ to recon-

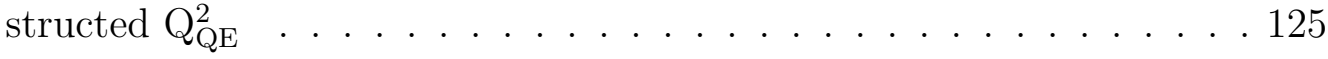

5.3 The unfolding matrix that maps reconstructed $\mathrm{Q}_{\mathrm{QE}}^{2}$ to true $\mathrm{Q}_{\mathrm{QE}}^{2} \ldots 125$

5.4 Uncertainties on the unfolding matrix that maps reconstructed $\mathrm{Q}_{\mathrm{QE}}^{2}$ to true $\mathrm{Q}_{\mathrm{QE}}^{2} \ldots \ldots \ldots \ldots . \ldots \ldots$

5.5 Flat selection efficiencies not accounted for in the simulation . . . . . 131

5.6 Product of efficiency and acceptance for each bin of $\mathrm{Q}_{\mathrm{QE}}^{2} \quad \ldots . . . \quad 132$

5.7 List of p-values from comparing events in different regions of MINOS 144

5.8 Weights for finding systematic error related to background dependence on neutrino energy . . . . . . . . . . . . . . . . . . 145

5.9 GENIE parameters and associated uncertainty . . . . . . . . 147 
5.10 Uncertainty on plane material composition . . . . . . . . . . . . . 164

5.11 Correlation matrix for the differential cross-section $\frac{\mathrm{d} \sigma}{\mathrm{d} \mathrm{Q}_{\mathrm{QE}}^{2}}$ on $\mathrm{CH} \ldots 166$

5.12 Fractional systematic uncertainties on $\frac{\mathrm{d} \sigma}{\mathrm{d} \mathrm{Q}_{\mathrm{QE}}^{2}} \ldots \ldots \ldots \ldots . \ldots . \ldots 167$

A.1 $\mathrm{X}$ and $\mathrm{Y}$ vertex resolution. . . . . . . . . . . . . . . . . . 191

A.2 Muon energy resolution binned by muon energy and angle . . . . . 192

A.3 Muon angle resolution binned by muon energy and angle . . . . . . . 195

A.4 Muon angle resolution in the XZ plane binned by muon energy and the XZ angle. . . . . . . . . . . . . . . . . . . . . . . 199

A.5 Muon angle resolution in the YZ plane binned by muon energy and the $\mathrm{YZ}$ angle. . . . . . . . . . . . . . . . . . 202

A.6 $\mathrm{Q}_{\mathrm{QE}}^{2}$ resolution, binned by $\mathrm{Q}_{\mathrm{QE}}^{2} \ldots \ldots \ldots \ldots \ldots \ldots \ldots \ldots$

B.1 Gains for the VST PMT . . . . . . . . . . . . . 210

B.2 TriP chip register values used for the VST . . . . . . . . . 214

B.3 Differences between MINER $\nu$ A and the VST . . . . . . . . . 215

B.4 The mapping of VST strips to electronics channels . . . . . . . . . 216

B.5 The charge to ADC ratio for the select VST FEB channels . . . . . 217

B.6 Single p.e. data for each VST channel . . . . . . . . . . . . . . 223

B.7 Low gain over high gain ADC ratios . . . . . . . . . . . . . 225

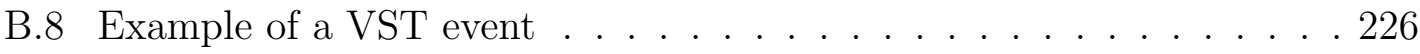

B.9 Number of p.e. per layer . . . . . . . . . . . . . . . . . . . 229

B.10 Light yield for different filter tests . . . . . . . . . . . . . . 232

B.11 Position resolution for different light yields . . . . . . . . . . . 232

B.12 Optimum VTH settings for each VST FEB . . . . . . . . . . 234 


\section{List of Figures}

1.1 Flavor composition of the neutrino mass eigenstates. . . . . . . . . . 14

1.2 Feynman diagram of $\nu_{\mu}+\mathrm{e}^{-} \rightarrow \mu^{-}+\nu_{\mathrm{e}} \ldots \ldots \ldots$. . . . . . 18

1.3 Feynman diagram of muon anti-neutrino CCQE scattering . . . . . . 20

1.4 NOMAD CCQE cross-section data with $\mathrm{M}_{\mathrm{A}}=1.05 \mathrm{GeV}$ comparison 24

1.5 MiniBooNE CCQE cross-section data with comparisons to several values of $\mathrm{M}_{\mathrm{A}}$.............................. 25

2.1 Beam facilities at Fermi National Accelerator Laboratory . . . . . . . 31

2.2 Distribution of MINER $\nu \mathrm{A}$ hits demonstrating bunch structure . . . . 33

2.3 Schematic of NuMI horns . . . . . . . . . . . . . . . 35

2.4 Schematic of the NuMI beam facility . . . . . . . . . . . . . . 37

2.5 Locations of hadron and muon monitors. . . . . . . . . . . . . . 38

3.1 Cross-sectional view of the MINER $\nu$ A detector . . . . . . . . . . . . 40

3.2 Orientation of the MINER $\nu \mathrm{A}$ coordinate system . . . . . . . . . . 41

3.3 Cross-sectional schematic of a MINER $\nu$ A module . . . . . . . . . . . 42

3.4 A diagram of MINER $\nu$ A Inner Detector scintillator strips . . . . . . . 45

3.5 MINER $\nu \mathrm{A}$ MINER $\nu \mathrm{A}$ PMT Box . . . . . . . . . . . . . . . . 49

3.6 MINOS Near Detector . . . . . . . . . . . . . 58 
3.7 ArgoNeuT detector . . . . . . . . . . . . . . . . . . 60

4.1 High gain pedestal $\ldots \ldots \ldots \ldots \ldots$

4.2 High gain pedestal with a background hit . . . . . . . . . . 63

4.3 Tri-linear fits to characterize gains for each channel on a Front End

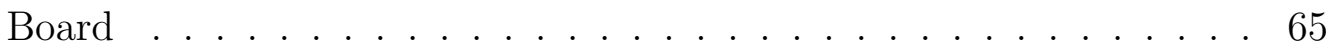

4.4 PMT gains for a particular time interval . . . . . . . . . . . 66

4.5 Result of calibration for variations between strips . . . . . . . . 68

4.6 Charge slewing as a function of the number of photoelectrons . . . . . 69

4.7 Timing resolution after slewing correction $\ldots \ldots \ldots \ldots$

4.8 Peak muon photoelectrons versus time $\ldots \ldots \ldots \ldots$. . . . . . 71

4.9 Data and simulation overlays of muon cluster energy after the MEU

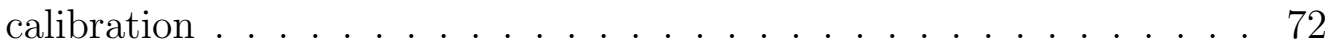

4.10 Plots of angle residuals between MINER $\nu \mathrm{A}$ and MINOS tracks in data 73

4.11 Plots of angle residuals between MINER $\nu \mathrm{A}$ and MINOS tracks in the simulation . . . . . . . . . . . . . . . 73

4.12 Plots searching for coordinate system scaling issues . . . . . . . 74

4.13 Plots searching for rotations in the XY plane between the MINER $\nu \mathrm{A}$

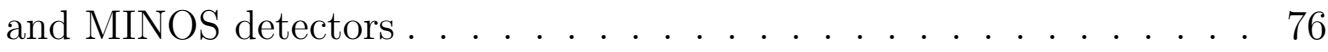

4.14 Central value reweighted flux plots . . . . . . . . . . . . . 79

4.15 Predicted energy loss for muons passing through ArgoNeuT _. . . . 83

4.16 A histogram of hits separated into time slices . . . . . . . . 85

4.17 An illustration of clusters formed from strips . . . . . . . . . 85

4.18 Illustration of tracking seed formation . . . . . . . . . . . . . . 89

4.19 Illustration of tracking candidate formation . . . . . . . . . . 90 
4.20 Plots of time difference for matching MINOS and MINER $\nu$ A tracks . 95

4.21 Plot of match residual for matching MINOS and MINER $\nu \mathrm{A}$ tracks . . 96

5.1 Muon energy and $\mathrm{Q}_{\mathrm{QE}}^{2}$ distributions for CC events . . . . . . . . . 104

5.2 Vetoed rock muon background event in MINER $\nu \mathrm{A}$. . . . . . . 106

5.3 Vetoed CCQE signal candidate event in MINER $\nu \mathrm{A} \ldots \ldots . . . .107$

5.4 Distributions of extra tracks and isolated blobs . . . . . . . . . 108

5.5 Recoil energy versus $\mathrm{Q}_{\mathrm{QE}}^{2}$ for signal and background and data/MC recoil energy overlay and ratio . . . . . . . . . . . . . . 110

5.6 Muon energy and $Q_{\mathrm{QE}}^{2}$ distributions for CCQE events . . . . . . . 111

5.7 Event display of a likely CCQE interaction in MINER $\nu \mathrm{A}$. . . . . . . 112

5.8 Event display of a likely CCQE interaction in MINER $\nu \mathrm{A} \ldots \ldots . . .113$

5.9 Efficiency and purity plots for CCQE selected events . . . . . . . 115

5.10 Estimated rock muon background distributions _ . . . . . . . 118

5.11 Recoil energy distributions prior to a background fit . . . . . . . 120

5.12 Recoil energy distributions after a background fit . . . . . . . . . 121

5.13 Background fit values . . . . . . . . . . . . . . . . . . 122

$5.14 \mathrm{Q}_{\mathrm{QE}}^{2}$ distribution and ratio prior to background subtraction . . . . . 122

$5.15 \mathrm{Q}_{\mathrm{QE}}^{2}$ distribution and ratio after background subtraction $\ldots \ldots . .123$

$5.16 \mathrm{Q}_{\mathrm{QE}}^{2}$ distribution and ratio after unfolding $\ldots \ldots \ldots \ldots \ldots$

$5.17 \mathrm{Q}_{\mathrm{QE}}^{2}$ versus neutrino energy acceptance plots . . . . . . . . 127

5.18 Neutrino energy distributions before and after analysis cuts for select

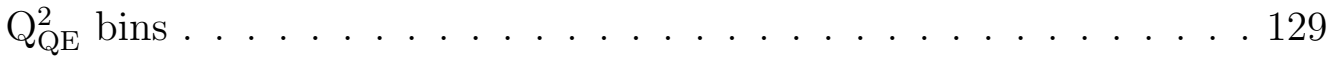

5.19 Neutrino energy distributions before and after analysis cuts for select

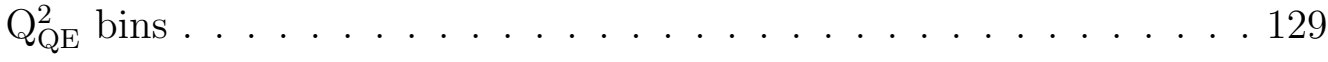


5.20 Neutrino energy distributions before and after analysis cuts for select

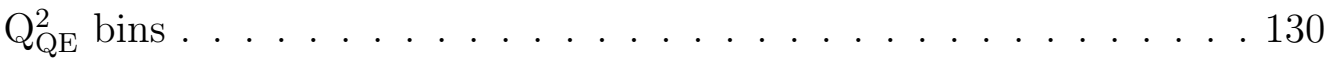

5.21 Neutrino energy distributions before and after analysis cuts for select

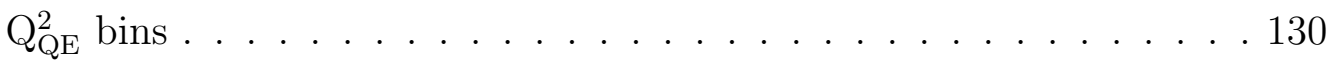

5.22 Neutrino energy distributions before and after analysis cuts for select

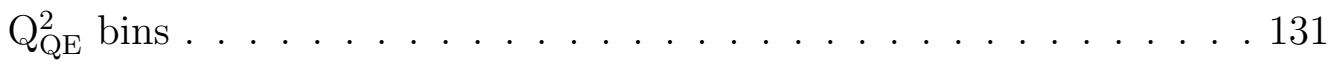

$5.23 \mathrm{Q}_{\mathrm{QE}}^{2}$ distribution and ratio after the efficiency correction $\ldots \ldots . .132$

5.24 The differential cross-section $\frac{\mathrm{d} \sigma}{\mathrm{dQ}_{\mathrm{QE}}^{2}}$ extracted on $\mathrm{CH}$ in MINER $\nu \mathrm{A}$ with statistical errors . . . . . . . . . . . . . . . . . 134

5.25 The difference of the inverses of momentum found in MINOS by range

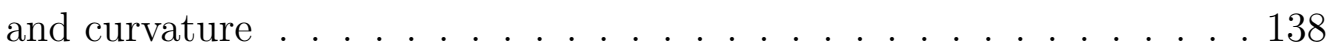

5.26 Angle residuals for broken tracks . . . . . . . . . . . . . 140

5.27 Position residuals for broken tracks . . . . . . . . . . . . . . . 141

5.28 Product of MINER $\nu$ A tracking efficiency and MINOS matching efficiency . . . . . . . . . . . . . . . . . . . . 142

5.29 The differential cross-section $\frac{\mathrm{d} \sigma}{\mathrm{dQ}_{\mathrm{QE}}^{2}}$ for different quadrants of MINOS . 144

5.30 Neutrino energy versus $Q_{\mathrm{QE}}^{2}$ and $\mathrm{W}$ versus $\mathrm{Q}_{\mathrm{QE}}^{2}$ for background . . . 146

5.31 Neutron-carbon inelastic and total cross-sections compared to Geant4 predictions . . . . . . . . . . . . . . . . . . . . 149

5.32 Neutron-iron inelastic and total cross-sections compared to Geant4 predictions . . . . . . . . . . . . . . 150

5.33 Neutron-iron inelastic and total cross-sections compared to Geant4 predictions . . . . . . . . . . . . . . 150

5.34 Recoil energy distributions with variations to neutron pathlength in simulation . . . . . . . . . . . . . . . . . 151 
5.35 Proton inelastic cross-sections compared to Geant4 predictions on carbon, iron, and lead .................... 152

$5.36 \pi^{+}$inelastic and total cross-sections compared to Geant4 predictions on carbon, iron, and lead . . . . . . . . . . . . . . . 153

$5.37 \pi^{-}$-carbon inelastic cross-section compared to Geant4 predictions . . 154

5.38 Positive and negative pion response found with the MINER $\nu \mathrm{A}$ test beam detector . . . . . . . . . . . . . . . . 155

5.39 Comparison of Michel electrons in data and simulation . . . . . . . 156

5.40 Example of a possible bremsstrahlung photon or delta ray from a muon 157

5.41 Recoil energy distributions before and after activity overlays in the simulation . . . . . . . . . . . . . . . . . . 158

5.42 Different components of the flux error . . . . . . . . . . . . 162

5.43 Example of a vetoed event that could be signal . . . . . . . . . . 165

5.44 Systematic errors by general type . . . . . . . . . . . . . . 166

5.45 Summary of systematic errors . . . . . . . . . . . . . 168

5.46 The differential cross-section $\frac{\mathrm{d} \sigma}{\mathrm{dQ}_{\mathrm{QE}}^{2}}$ extracted on $\mathrm{CH}$ in MINER $\nu \mathrm{A}$ with statistical and systematic errors . . . . . . . . . . . 168

5.47 The differential cross-section $\frac{\mathrm{d} \sigma}{\mathrm{dQ}_{\mathrm{QE}}^{2}}$ extracted on $\mathrm{CH}$ in MINER $\nu \mathrm{A}$ compared to other simulation models . . . . . . . . . . . . 170

A.1 X Vertex Distributions . . . . . . . . . . . . . . . . 184

A.2 Y Vertex Distributions . . . . . . . . . . . . . . . . 185

A.3 Module vertex ditributions . . . . . . . . . . . . . . 186

A.4 X coordinate of the MINER $\nu \mathrm{A}$ track projection to the face of MINOS 187

A.5 Y coordinate of the MINER $\nu \mathrm{A}$ track projection to the face of MINOS 188 
A.6 Absolutely normalized muon angle distributions _ . . . . . . . . 188

A.7 Absolutely normalized muon energy overlay and ratio . . . . . . . 189

A.8 Neutrino energy distributions and ratios . . . . . . . . . . . 189

A.9 Absolutely normalized $\mathrm{Q}_{\mathrm{QE}}^{2}$ energy overlay and ratio . . . . . . . . 190

A.10 $\mathrm{Q}_{\mathrm{QE}}^{2}$ plots for two different regions of neutrino energy . . . . . . . . 190

A.11 X and $\mathrm{Y}$ vertex resolution plots . . . . . . . . . . . . . 191

A.12 Muon energy resolution plots for muons angles from 0 to 5 degrees . . 192

A.13 Muon energy resolution plots for muons angles from 0 to 5 degrees . . 193

A.14 Muon energy resolution plots for muons angles from 0 to 5 degrees . . 194

A.15 Muon angle resolution for muons with an energy of 0 to $3 \mathrm{GeV}$. . . 195

A.16 Muon angle resolution for muons with an energy of 3 to $5 \mathrm{GeV}$. . . 196

A.17 Muon angle resolution for muons with an energy greater than $5 \mathrm{GeV} 197$

A.18 Muon angle resolution in the XZ plane for muons with an energy of

0 to $3 \mathrm{GeV} \ldots \ldots \ldots \ldots \ldots$. . . . . . . . . . . . . . . . . . . . . .

A.19 Muon angle resolution in the XZ plane for muons with an energy of

3 to $5 \mathrm{GeV} \ldots \ldots \ldots \ldots . \ldots \ldots$

A.20 Muon angle resolution in the XZ plane for muons with an energy greater than $5 \mathrm{GeV} \ldots \ldots \ldots \ldots \ldots$

A.21 Muon angle resolution in the YZ plane for muons with an energy of 0 to $3 \mathrm{GeV} \ldots \ldots \ldots \ldots \ldots$. . . . . . . . . . . . . . . . . . . .

A.22 Muon angle resolution in the YZ plane for muons with an energy of

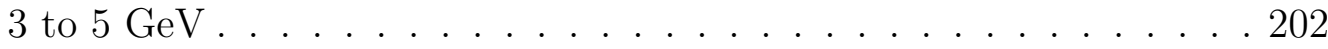

A.23 Muon angle resolution in the YZ plane for muons with an energy greater than $5 \mathrm{GeV} \ldots \ldots \ldots \ldots$

A.24 $\mathrm{Q}_{\mathrm{QE}}^{2}$ resolution plots . . . . . . . . . . . . . . . 204 


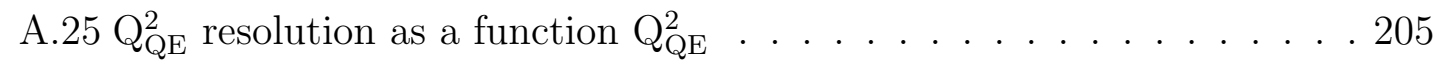

B.1 Picture and schematic of the Vertical Slice Test detector . . . . . 207

B.2 Picture of CALDET box that houses the VST PMT . . . . . . . 208

B.3 Picture of the FEBs used for the VST . . . . . . . . . . . . 211

B.4 Schematic of the TriP chip . . . . . . . . . . . . . . 212

B.5 Plots of high and low gain pedestals . . . . . . . . . . . 218

B.6 FEB pedestal average versus single channel pedestal . . . . . . . . 220

B.7 Results for finding the optimum common mode noise correction . . . 221

B.8 Single p.e. fit example . . . . . . . . . . . . . . . . . . . 223

B.9 High gain ADC versus low gain ADC for cosmic ray muons . . . . . 225

B.10 Light yield for a layer . . . . . . . . . . . . . . . . . 228

B.11 Plot of position residual . . . . . . . . . . . . 230

B.12 Plot of position residual versus position . . . . . . . . . . . . . 231

B.13 Diagram of potential gap between strips $\ldots \ldots \ldots . \ldots 232$

B.14 Position resolution as a function of light yield $\ldots \ldots \ldots . . \ldots 233$

B.15 Plot of timing resolution . . . . . . . . . . . . 236

B.16 Plots demonstrating charge slewing $\ldots \ldots \ldots \ldots \ldots$ 


\section{Chapter 1}

\section{Introduction}

One of the most significant discoveries of particle physics in recent years is the ability of neutrinos to oscillate from one flavor to another as they propagate through space. An implication of this discovery is that neutrinos have mass and that a particular flavor state is composed of a superposition of mass eigenstates states. This discovery created a new branch of experimental particle physics to study neutrino oscillations and generated many new questions. What are the details of this mixing? What fundamental mechanism gives neutrinos mass? Do neutrinos experience CP violation? Are there sterile neutrinos? Could neutrinos be their own anti-particles? Could they be responsible for leptogenesis, which created the matter-anti-matter asymetry that we observe in the universe? Physicists hope to answer these questions through current and future experiments.

However, current knowledge of neutrino-nucleus interactions limit the precision of measurements that such experiments can make. Data on neutrino cross-sections, particularly data for specific final states, have large errors. Nuclear effects, which can modify scattering cross-sections as well as final state kinematics, are not fully 
understood. Changes in the final state kinematics can affect the energy reconstruction for an interaction, something that can be a large problem for neutrino oscillation experiments. The dependence of neutrino cross-sections on atomic number is virtually unexamined. Due to the current state of knowledge of neutrino interactions, models of those interactions have large errors and do not include certain effects. As a result, this limits the precision of oscillation experiments.

This has motivated many new measurements of neutrino-nucleus interactions. In particular, the MINER $\nu \mathrm{A}$ (Main INjector ExpeRiment $\nu \mathrm{A}$ ) experiment is being conducted to study such interactions for neutrinos in the several GeV region, a region which if of particular interest to neutrino oscillation experiments. We

present a measurement of the differential cross-section $\frac{\mathrm{d} \sigma}{\mathrm{dQ}^{2}}$ for Charged Current muon anti-neutrino Quasi-Elastic (CCQE) scattering from the MINER $\nu \mathrm{A}$ experiment. A description of the current understanding of this interaction and our current understanding of neutrinos and the physics of their interactions follow.

\subsection{The Standard Model}

The Standard Model of particle physics is a framework which describes the fundamental particles we find in nature and the forces by which they interact with the exception of gravity. The Standard Model can be represented by a $\mathrm{SU}(3) \otimes \mathrm{SU}(2)_{L} \otimes \mathrm{U}(1)$ group, which describes the types of interactions that exist within the theory [1].

\subsubsection{Elementary Particles}

The theory describes fundamental particles that are either fermions or bosons. Fermions have half-integer spin while bosons have integer spin. Fermions are so 
named since they obey Fermi statistics while bosons obey Bose-Einstein statistics. Fermions also obey the Dirac equation [2],

$$
\left(\mathrm{i \hbar} \gamma^{\mu} \frac{\partial}{\partial \mathrm{x}_{\mu}}-\mathrm{mc}\right) \psi=0
$$

where the term $\psi$ is the four component spinor field describing a fermion and $\gamma^{\mu}$ are the Dirac matrices. One consequence of Fermi-statistics is that two identical fermions with the same quantum numbers cannot occupy the same state. This is more commonly known as the Pauli exclusion principle. The fundamental fermions we find in nature come in two classes, leptons and quarks. These particles differ by their mass, charge, and the fundamental interactions by which they interact. Each fermion has an anti-particle. An anti-particle is exactly the same as a its corresponding particle, but has the opposite electromagnetic charge or in the case of neutrinos, helicity. Neutrinos may be their own anti-particle where an anti-neutrino is a neutrino, but with opposite helicity [1]. Of the fundamental fermions, the lightest two quarks and the lightest charged lepton make up everyday matter.

We find two varieties of fundamental bosons in nature. The exchange of vector gauge bosons mediates three of the four fundamental forces we find in nature: electromagnetism, the weak force, and the strong force. The theory also predicts a boson related to the Higgs mechanism which gives particles mass. A particle consistent with the Higgs boson has been found recently by experiments at the LHC. The Standard Model requires that this particle have 0 spin, referred to as a scalar. Currently, experimental evidence can only estabish this is a boson with spin 0 or spin $2[3]$ [4]. Each uncharged boson is its own anti-particle. 


\begin{tabular}{|c|c|c|c|c|}
\hline Type of Particle & Spin & Charge & Interacts Strongly? & Mass \\
\hline \multicolumn{5}{|l|}{ Quarks } \\
\hline $\mathrm{u}$ & $1 / 2$ & $2 / 3$ & Yes & $2.3 \mathrm{MeV} / c^{2}$ \\
\hline d & $1 / 2$ & $-1 / 3$ & Yes & $4.8 \mathrm{MeV} / c^{2}$ \\
\hline $\mathrm{c}$ & $1 / 2$ & $2 / 3$ & Yes & $1.275 \mathrm{GeV} / c^{2}$ \\
\hline $\mathrm{s}$ & $1 / 2$ & $-1 / 3$ & Yes & $95 \mathrm{MeV} / c^{2}$ \\
\hline $\mathrm{t}$ & $1 / 2$ & $2 / 3$ & Yes & $173.5 \mathrm{GeV} / c^{2}$ \\
\hline $\mathrm{b}$ & $1 / 2$ & $-1 / 3$ & Yes & $4.65 \mathrm{GeV} / c^{2}$ \\
\hline \multicolumn{5}{|l|}{ Leptons } \\
\hline $\mathrm{e}$ & $1 / 2$ & -1 & No & $0.5486 \mathrm{MeV} / c^{2}$ \\
\hline$\nu_{e}$ & $1 / 2$ & 0 & No & $\sum_{\alpha=e, \mu, \tau} m_{\nu_{\alpha}}<0.3-1.5 \mathrm{eV} / c^{2}$ \\
\hline$\mu$ & $1 / 2$ & -1 & No & $105.7 \mathrm{MeV} / c^{2}$ \\
\hline$\nu_{\mu}$ & $1 / 2$ & 0 & No & $\sum_{\alpha=e, \mu, \tau} m_{\nu_{\alpha}}<0.3-1.5 \mathrm{eV} / c^{2}$ \\
\hline$\tau$ & $1 / 2$ & -1 & No & $1.777 \mathrm{GeV} / c^{2}$ \\
\hline$\nu_{\tau}$ & $1 / 2$ & 0 & No & $\sum_{\alpha=e, \mu, \tau} m_{\nu_{\alpha}}<0.3-1.5 \mathrm{eV} / c^{2}$ \\
\hline
\end{tabular}

Table 1.1: A list of the three generations of fundamental fermions and their properties. Only limits exist on the masses of neutrinos [5].

\subsubsection{Quarks}

Quarks come in three generations, with each generation being composed of two different types (also known as flavors) of quarks for a total of six. The first generation is composed of up (u) and down (d), the second of charm (c) and strange (s), and the third of top (t) and bottom (b). Generations of quarks differ from each other by mass. Quarks can interact through all known fundamental forces. They are the only fundamental fermions that interact via the strong force and have a property known as color, which is the type of effective charge associated with the strong force. Quarks have fractional electromagnetic charges. The properties of the six quarks are listed in Table 1.1.

Particles composed of quarks are known as hadrons. The property of color determines the type of particles that can be formed by quarks. There are two 
general types of hadrons, mesons and baryons. Mesons are generally made up of a quark and an anti-quark. No stable forms of mesons exist in nature. Baryons are composed of either three quarks or three anti-quarks. The protons and neutrons found in atomic nuclei are types of baryons. The total number of baryons found in the universe is conserved within the Standard Model.

Baryons heavier than the proton and mesons often decay weakly. In the case of charged pions, the decay is generally $\pi^{ \pm} \rightarrow \mu^{ \pm}+\bar{\nu}_{\mu}\left(\nu_{\mu}\right)$. For mesons and baryons composed of heavier quarks, the heavy quarks decay weakly to light quarks. The weak force mixes quark flavors as described by the Cabibbo-Kobayashi-Maskawa (CKM) matrix [6].

Both mesons and baryons are color-neutral. Neither naked color or naked quarks have been observed in nature. This is due to the nature of the strong force, described in Section 1.1.4.

\subsubsection{Leptons}

Three generations of leptons exist, each generation being composed of a charged lepton and a neutrino. The first generation is composed of an electron (e) and electron-neutrino $\left(\nu_{e}\right)$, the second of a muon $(\mu)$ and muon-neutrino $\left(\nu_{\mu}\right)$, and the third of a tau $(\tau)$ and tau neutrino $\left(\nu_{\tau}\right)$. Properties of leptons are listed in Table 1.1. Like quarks, particles of each generation differ in mass. Unlike quarks, leptons are associated with another property, lepton number, conserved by interactions within the Standard Model. Lepton number is conserved separately for each generation. Lepton number is found by associating +1 for a charged lepton or its neutrino partner and -1 for an anti-matter charged lepton or its anti-neutrino partner. For example, muons typically undergo the decay $\mu^{-} \rightarrow e^{-}+\bar{\nu}_{e}+\nu_{\mu}$. In this case, the 
$\mu^{-}$has muon lepton number 1 . In the decay, a $\nu_{\mu}$ is created which also has muon lepton number of 1 . In this case, the muon lepton number is 1 before and after the decay. An electron is also involved in the decay and necessary to conserve charge. The electron has a electron lepton number of 1 . If a $\bar{\nu}_{e}$ were not also created in the decay, which has electron lepton number of -1, the decay would not conserve lepton number. Since a $\bar{\nu}_{e}$ is created, the total electron number is 0 before the decay and is $1-1=0$ after the decay. Note that neutrino oscillation, described in Section 1.2, violates lepton number. Despite this violation, the actual Standard Model interactions conserve lepton number.

\section{Charged Leptons}

Charged leptons interact via the electromagnetic force, the weak force, and gravity. Each charged lepton has an anti-particle with the opposite charge. The lightest charged lepton, the electron, is a constituent of the atom.

\section{Neutrinos}

Neutrinos are neutral and interact through the weak force and gravity. Only lefthanded ( $\mathrm{LH})$ neutrinos and right-handed ( $\mathrm{RH})$ anti-neutrinos are created in Standard Model interactions. In the Standard Model, RH neutrinos and LH antineutrinos would not couple weakly. If boosted into the proper reference frame, LH neutrinos would appear as RH neutrinos and RH anti-neutrinos as LH antineutrinos to an observer at rest in that reference frame. This is fairly impractical given the tiny mass of neutrinos. This would not be possible if neutrinos were massless, but the discovery of neutrino oscillation established that neutrinos do in fact have mass. 
With the discovery of neutrino oscillations, physicists find that the neutrino flavor eigenstates that are created in neutrino interactions are not the same as the neutrino mass eigenstates. Superpositions of the more fundamental mass eigenstates can form a flavor eigenstate. The mass eigenstates are identified with the labels $\nu_{1}$, $\nu_{2}$, and $\nu_{3}$. The flavor composition of mass eigenstates can be probed by studying neutrino oscillations which we discuss in Section 1.2. The masses of the neutrino mass eigenstates are not known at this time, but the differences in the square of the masses can also be extracted from neutrino oscillation experiments. Currently, limits exist on the masses of neutrino flavor eigenstates which are listed in Table 1.1.

Experimentalists have yet to determine whether neutrinos are Majorana particles. A Majorana particle is its own anti-particle. The most promising method for resolving this is by looking for neutrinoless double beta decay $(0 \nu \beta \beta)$ in certain radioactive elements. The elements of interest are able to undergo two neutrino double beta decay $\nu \nu \beta \beta$. If neutrinos are Majorana particles, it may be possible for neutrinos to annihilate during a decay leaving a two electron final state. Conclusive observation of such a decay would establish not only that neutrinos are Majorana particles, but would also allow a direct measurement of the electron neutrino mass [7].

\subsubsection{Fundamental Interactions}

The Standard Model describes the dynamics of three fundamental forces, electromagnetism, the weak force, and the strong force. Each force is described in terms of the exchange of gauge bosons. Table 1.2 lists the fundamental bosons described by the Standard Model along with various properties of each particle. 


\begin{tabular}{c|cccc}
\hline \hline Boson & Spin & Charge & Mass $\left(\mathrm{GeV} / c^{2}\right)$ & Role \\
\hline photon & 1 & 0 & 0 & Mediate electromagnetic force \\
$\mathrm{W}^{ \pm}$ & 1 & \pm 1 & 80.385 & Mediate charge current weak interactions \\
$\mathrm{Z}$ & 1 & 0 & 91.188 & Mediate neutral current weak interactions \\
gluons & 1 & 0 & 0 & Mediate strong nuclear force \\
Higgs & 0 & 0 & $\approx 125$ & Excitation in the Higgs field \\
\hline \hline
\end{tabular}

Table 1.2: A list of the fundamental bosons described by the Standard Model [3] [4] [5].

\section{Electromagnetism and the Weak Force}

Electromagnetism is mediated by the photon. Photons are massless and couple to charged particles. Electromagnetic interactions at the quantum level were first successfully described by Quantum Electrodynamics (QED) [1]. Due to the masslessness of the photon, the range of this force is effectively infinite. Much of the everyday phenomena observed by people are the result of this force.

The weak force is mediated by massive self-coupling gauge bosons, the $\mathrm{W}^{ \pm}$and $\mathrm{Z}$, which due to their large mass act on only very short scales. The short range is due to the Heisenberg Uncertainty Principle which specifies that $\Delta \mathrm{E} \Delta \mathrm{t} \geq \frac{\hbar}{2}$. This means a massive virtual weak boson may be exchanged in a low energy reaction, but may only act for short periods of time and consequently only over very short distances. The weak force is responsible for some phenomena such as radioactive beta decay and fusion reactions that occur within the sun.

The weak force is unique in that it is fundamentally parity violating. The strong force and electromagnetism both conserve parity. It has also been found that the combined symmetry of charge conjugaton and parity, CP symmetry, can also be violated in weak interactions [1]. CP violation manifests as a slight difference in behavior between matter and anti-matter. CP violation has been observed in quark mixing and is suspected to be present in neutrino mixing. 


\section{Electroweak Theory}

In the 1960s, Electroweak theory emerged which described the weak and electromagnetic forces as different aspects of one phenomenon, the Electroweak force [1]. The theory describes the $\mathrm{W}^{ \pm}, \mathrm{Z}$, and photon as massless particles. However, something known as the Higgs mechanism was shown to allow for spontaneous symmetry breaking giving the $\mathrm{W}^{ \pm}$and $\mathrm{Z}$ bosons mass through coupling to the Higgs field [1]

This theory makes several very bold predictions. It predicts the existence of $\mathrm{W}^{ \pm}$ and $\mathrm{Z}$ bosons and neutral current interactions none of which had been observed at the time of the prediction. It predicts that the weak force and electromagentic force should behave with increasing similarity as interaction energies increase toward the energy scale set by the mass of the $\mathrm{W}^{ \pm}$and $\mathrm{Z}$ bosons. It also describes a mechanism by which particles acquire mass. This occurs through particles coupling to something called the Higgs field. Additionally, an excitation in the Higgs field, known as the Higgs boson, is also predicted.

Neutral current interactions have since been observed [1]. Physicists discovered the $\mathrm{W}^{ \pm}$and $\mathrm{Z}$ bosons in the 1980s [8] [9]. The similarity in the two forces at high energies has also been verified. Last, a particle consistent with the Higgs boson has recently been observed by experiments operating at the LHC [3] [4].

\section{The Strong Force}

The strong force is described by Quantum Chromodynamics (QCD). This theory describes the strong force as being mediated by massless gluons. These gluons are self-coupling and very strongly interacting. This means that gluons couple to each other as they are being exchange by quarks. Consequently, hadronic particles tend to be very complex in regard to the dynamics occuring within the particle. 
The strong force has a peculiar behavior worth noting. The strong force decreases in strength when two quarks are very close or when energies involved in the interaction are very high. Additionally, the force increases in strength as bound quarks are separated. This behavior is known as asymptotic freedom [6]. An implication of this effect is that at very high energies, pertubation theory can be used to make predictions and conversely at low energies it cannot. As a result, calculations at lower energies, in particular in the few GeV region, can be difficult. The interaction cannot be considered as between point like objects and other methods must be employed such as lattice QCD or the use of structure functions and form factors.

\subsubsection{Limitations of the Standard Model}

Despite the success of the Standard Model, there are observations and phenomena that it does not describe. The model does not describe gravity. There are several independent pieces of evidence for dark matter, which is necessary to account for the observed rotation of galaxies. The Standard Model does not include any particle that has the properties consistent with dark matter [10]. Additionally, observations have found that the expansion of the universe appears to be accelerating, the hypothetical cause of which we refer to as dark energy [11]. The Standard Model does not predict

a dark energy mechanism. Last, the Standard Model does not describe the origin of neutrino mass, why particles have a particular mass, the cause of $\mathrm{CP}$ violation, or why there are three generations of particles. 


\subsection{Neutrino Oscillation}

As discussed in Section 1.1.3, we now know that neutrino flavor eigenstates are not the same as the neutrino mass eigenstates. A consequence of this is that as neutrinos propagate, the superposition of mass eigenstates that compose a flavor eigenstate has a time dependent interference pattern that leads to a varying probability that a particular flavor eigenstate will be observed. This mixing is better known as neutrino oscillation.

We know that a superposition of at least three mass eigenstates is needed to describe the mixing. However, there is evidence for mixing involving one or more sterile neutrinos from the Liquid Scintillator Neutrino Detector (LSND) [12] and MiniBooNE experiments [13]. Results from cosmology potentially allow an additional sterile neutrino as well [14]. Here sterile refers to the lack of coupling by any Standard Model interaction.

We know there are only three flavor eigenstates that need to be considered in describing neutrino oscillation from studies of $\mathrm{Z}$ decays at LEP [5]. LEP established that there are no more than three weakly coupling neutrinos with less than half the mass of the $\mathrm{Z}$ boson. Flavored neutrinos heavier than the $\mathrm{Z}$ boson mass are disfavored by cosmology [5].

The Pontecorvo-Maki-Nakagawa-Sakata (PMNS) matrix [15] details the three flavored eigenstates in terms of a superposition of three mass eigenstates in Equation 1.3. A commonly used form of this matrix for three generations is

$$
\left(\begin{array}{ccc}
\mathrm{c}_{12} \mathrm{c}_{13} & \mathrm{~s}_{12} \mathrm{c}_{13} & \mathrm{~s}_{13} \mathrm{e}^{-\mathrm{i} \delta} \\
-\mathrm{s}_{12} \mathrm{c}_{23}-\mathrm{c}_{12} \mathrm{~s}_{23} \mathrm{~s}_{13} \mathrm{e}^{\mathrm{i} \delta} & \mathrm{c}_{12} \mathrm{c}_{23}-\mathrm{s}_{12} \mathrm{~s}_{23} \mathrm{~s}_{13} \mathrm{e}^{\mathrm{i} \delta} & \mathrm{s}_{23} \mathrm{c}_{13} \\
\mathrm{~s}_{12} \mathrm{~s}_{23}-\mathrm{c}_{13} \mathrm{c}_{23} \mathrm{~s}_{13} \mathrm{e} \mathrm{e}^{\mathrm{i} \delta} & -\mathrm{c}_{12} \mathrm{~s}_{23}-\mathrm{s}_{12} \mathrm{c}_{23} \mathrm{~s}_{13} \mathrm{e}^{\mathrm{i} \delta} & \mathrm{c}_{23} \mathrm{c}_{13}
\end{array}\right)
$$


with $\mathrm{s}_{\mathrm{ij}}=\sin \theta_{\mathrm{ij}}, \mathrm{c}_{\mathrm{ij}}=\cos \theta_{\mathrm{ij}}$ and $\theta_{\mathrm{ij}}=\theta_{12}, \theta_{23}$, or $\theta_{13}$. These are the neutrino mixing angles that determine the combination of mass eigenstates needed to make a neutrino flavor eigenstate. The term $\delta$ is the $\mathrm{CP}$ violating phase. The PMNS matrix is generally multiplied by a diagonal matrix containing two Majorana phases, but this matrix is not shown here. These phases could be present if neutrinos are Majorana particles. The Majorana phases cannot affect neutrino oscillation, although they can have an impact on lepton number conservation [16].

The CP violating phase is of great interest. CP violation is the only known asymmetry between matter and anti-matter. This asymmetry is suspected to be the cause of the matter-anti-matter asymmetry we find in the universe. Currently, $\mathrm{CP}$ violation has only been observed in the quark sector, but quantitately is too small to give rise to the present imbalance. $\mathrm{CP}$ violation in the neutrino sector could be the source of this imbalance, a phenomenon which is refered to as leptogenesis [7].

Given the PMNS matrix, it is possible to construct neutrino flavor eigenstates from the neutrino mass eigenstates

$$
\nu_{\mathrm{l}}=\sum_{\mathrm{i}=1,2,3} \mathrm{U}_{\mathrm{li}} \nu_{\mathrm{i}}, \mathrm{l} \in \mathrm{e}, \mu, \tau
$$

where the term $\mathrm{U}_{\mathrm{li}}$ is the PMNS matrix described in Equation 1.2, $\nu_{i}$ is a neutrino mass eigenstate, and $\nu_{1}$ is a neutrino flavor eigenstate. This, however, is not sufficient to describe neutrino oscillation. Neutrino oscillation is dependent upon several other terms. The probability of oscillation of one type of neutrino to another assuming oscillation between only two flavors is given by [17]

$$
\mathrm{P}\left(\nu_{\alpha} \rightarrow \nu_{\beta}\right)=\sin ^{2}(2 \theta) \sin ^{2}\left(\frac{1.267 \Delta \mathrm{m}_{\mathrm{ij}}^{2}\left(\mathrm{eV}^{2}\right) \mathrm{L}(\mathrm{km})}{\mathrm{E}(\mathrm{GeV})}\right)
$$


where $\nu_{\alpha}$ and $\nu_{\beta}$ are neutrino flavor eigenstates. The term $\theta$ is the relevant mixing angle that characterizes the two neutrino oscillation model. The value $\mathrm{L}$ is the pathlength of the neutrino in kilometers, $\mathrm{E}$ is the energy of the neutrino in units of $\mathrm{GeV}$, and $\Delta \mathrm{m}_{\mathrm{ij}}^{2}$ is the difference of the square of the two neutrino mass eigenstates, or $\mathrm{m}_{\mathrm{i}}^{2}-\mathrm{m}_{\mathrm{j}}^{2}$, being considered in the oscillation model. Although three neutrino mixing is slightly more complicated than the model above, the oscillations are depenent upon the same general quantities. Oscillations depend upon the ratio $\frac{\mathrm{L}}{\mathrm{E}}$, which experimenters can control, the term $\Delta m_{i j}^{2}$, and the neutrino mixing angles $\theta_{12}$, $\theta_{23}$, and $\theta_{13}$. The $\mathrm{CP}$ violating phase should also be observable in certain types of oscillation. Values of the $\sin ^{2} 2 \theta_{\mathrm{ij}}$ for each mixing angle along with the values of $\Delta \mathrm{m}_{\mathrm{ij}}^{2}$ are given in Table 1.3. Note that for two of the mass splitting terms, $\Delta \mathrm{m}_{13}^{2}$ and $\Delta \mathrm{m}_{23}^{2}$, the sign of these are not known. Specifically, it is not known whether $\nu_{3}$ is the lightest or heaviest neutrino mass eigenstate. Solar neutrino observations establish that the $\nu_{2}$ eigenstate is heavier than $\nu_{1}$ eigenstate [5]. The scenario where $\nu_{3}$ is the lightest neutrino mass eigenstate is referred to as an inverted neutrino mass hierarchy [5]. Figure 1.1 depicts the neutrino mass eigenstates and their relative flavor composition.

A variety of experiments in the past 15 years have supported the three neutrino mixing model and have measured many of the parameters that go into that model. The first evidence for neutrino oscillation came from the Homestake Experiment, which found roughly a third fewer neutrinos generated by the sun (solar neutrinos) than predicted given the rate of fusion reactions [18]. Super Kamiokande measured neutrino oscillation in neutrinos generated from cosmic ray interactions in the atmosphere (atmospheric neutrinos) [19]. The Super Kamiokande results were verified by two accelerator based experiments, KEK to Kamioka (K2K) [20] and 


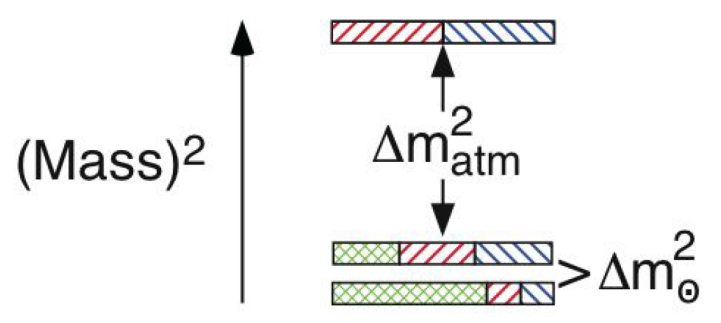

Figure 1.1: Flavor composition of the neutrino mass eigenstates ordered in the normal hierachy. In this depiction, the heaviest eigenstate is $\nu_{3}$, the second heaviest is $\nu_{2}$ and the lightest is $\nu_{1}$. Flavor composition is depicted for each neutrino mass-eigenstate with the $\nu_{e}$ component as cross-hatched lines (green), the $\nu_{\mu}$ component as right-leaning lines (red), and the $\nu_{\tau}$ component as left-leaning lines (blue). Due to results from Daya Bay [24] and RENO [25], we now know that $\nu_{3}$ has a small $\nu_{e}$ component. The term $\Delta m_{32}^{2}$ is represented as $\Delta m_{a t m}^{2}$ due to the initial measurment of this value with atmospheric neutrinos and the term $\Delta m_{21}^{2}$ is represented as $\Delta m_{\odot}^{2}$ due to initial measurements of this value in solar neutrino experiments. Note that in the case of the inverted hierarchy, $\nu_{3}$ (on top) would be less massive than the other two neutrino mass eigenstates. Figure from the PDG [5].

\begin{tabular}{c|c}
\hline \hline Parameter & Value \\
\hline $\sin ^{2} 2 \theta_{12}$ & $0.857_{-0.025}^{+0.023}$ \\
$\sin ^{2} 2 \theta_{23}$ & $>0.95(90 \%$ confidence $)$ \\
$\sin ^{2} 2 \theta_{13}$ & $0.098 \pm 0.013$ \\
$\Delta \mathrm{m}_{12}^{2}$ & $7.5_{-0.20}^{+0.19} \times 10^{-5} \mathrm{eV}^{2}$ \\
$\left|\Delta \mathrm{m}_{23}^{2}\right|$ & $2.32_{-0.08}^{+0.12} \times 10^{-3} \mathrm{eV}^{2}$ \\
$\left|\Delta \mathrm{m}_{13}^{2}\right|$ & $2.32_{-0.08}^{+0.12} \times 10^{-3} \mathrm{eV}^{2}$ \\
$\delta$ & Unknown \\
\hline \hline
\end{tabular}

Table 1.3: The current best measurements of different parameters that affect neutrino oscillation based on recent PDG values which includes data from RENO and Daya Bay. The CP violating phase $\delta$ has not been measured. Note that the values of $\Delta \mathrm{m}_{13}^{2}$ and $\Delta \mathrm{m}_{23}^{2}$ cannot be distinguished at this time nor is it known whether these particular parameters are positive or negative [5]. 
Main Injector Neutrino Oscillation Search (MINOS) [21]. Data from the Sudbury Neutrino Observatory (SNO) helped confirm that neutrino mixing is the explantion for the solar neutrino deficit [22]. Kamioka Liquid Scintillator Antineutrino Detector (KamLAND) further bolstered the three neutrino mixing model by observing neutrino oscillations in a similar regime as solar neutrinos, but using reactor antineutrinos [23]. We know the value of $\theta_{13}$ from recent measurements by the Daya Bay [24] and RENO [25] experiments.

Many unanswered questions remain. We currently do not know the absolute masses of the neutrinos, the neutrino mass hierarchy, whether sterile neutrinos exist, whether neutrinos are Majorana or Dirac particles, or whether $\mathrm{CP}$ violation exists within the neutrino sector. Experiments such as the Enriched Xenon Observatory (EXO) [26], Neutrino Ettore Majorana Observatory (NEMO) [27], and Majorana [28] are searching for $00 \nu \beta$ which would establish neutrinos as Majorana particles if observed. The Karlsruhe Tritium Neutrino (KATRIN) experiment is studying the final state electron from beta decay of tritium to directly measure the mass of the electron neutrino eigenstate, with projected sensitivity down to 0.2 $\mathrm{eV}[29]$

Other questions require dedicated accelerator neutrino experiments to answer. Accelerator based oscillation experiments have already contributed to knowledge of neutrino mixing parameters and appear as the best candidate for measuring the neutrino mass hierarchy, searching for $\mathrm{CP}$ violation, as well as continuing the search for sterile neutrinos. Several accelerator experiments look to contribute to this area such as the Tokai to Kamioka (T2K) experiment [30], the NuMI Off-Axis $\nu_{e}$ Appearance (NOvA) experiment [31], and the Oscillation Project with EmulsiontRacking Apparatus (OPERA) experiment [32]. For such accelerator based neutrino 
experiments, baselines and the corresponding neutrino energies require operating in the few $\mathrm{GeV}$ region. In this region, errors on neutrino cross-sections are large and sometimes contradictory and nuclear effects important. This has motivated the study of such topics by oscillation experiments and dedicated experiments such as $\operatorname{MINER} \nu \mathrm{A}$.

\section{$1.3 \quad$ Neutrino Scattering}

A common quantity of interest in particle physics is the cross-section. For two body elastic scattering, the differential cross-section can be calculated using the Feynman rules [33], such that

$$
\mathrm{d} \sigma=|\mathcal{M}|^{2} \frac{(2 \pi)^{4}}{4 \sqrt{\mathrm{p}_{1} \cdot \mathrm{p}_{2}-\mathrm{m}_{1} \mathrm{~m}_{2}}} \delta^{(4)}\left(\mathrm{p}_{3}+\mathrm{p}_{4}-\mathrm{p}_{1}-\mathrm{p}_{2}\right) \frac{\mathrm{d}^{3} \mathrm{p}_{3}}{(2 \pi)^{3} 2 \mathrm{E}_{3}} \frac{\mathrm{d}^{3} \mathrm{p}_{4}}{(2 \pi)^{3} 2 \mathrm{E}_{4}}
$$

where $\mathcal{M}$ is the matrix amplitude which is determined by the particulars of the interaction. This matrix amplitude depend upon the particles involved, how they interact, and whether there are several ways this final state can be achieved. We use the natural Heaviside Lorentz units where $c=\hbar=1$. Below follows a derivation by [33]. For point-like particles, this amplitude can be calculated analytically. For instance, for a muon neutrino scattering off of an electron via $\mathrm{W}$ exchange $\left(\nu_{\mu}+\mathrm{e}^{-}\right.$ $\left.\rightarrow \mu^{-}+\nu_{e}\right)$ like in Figure 1.2, we find the matrix element to be

$$
\mathcal{M}=\frac{\mathrm{g}_{\mathrm{W}}^{2}}{8}\left(\overline{\mathrm{u}}(3) \gamma^{\mu}\left(1-\gamma^{5}\right) \mathrm{u}(1)\right) \frac{-\mathrm{i}\left(\mathrm{g}_{\mu \nu}-\frac{\mathrm{q}_{\mu} \mathrm{q}_{\nu}}{\mathrm{M}_{\mathrm{W}}^{2}}\right)}{\mathrm{q}^{2}-\mathrm{M}_{\mathrm{W}}^{2}}\left(\overline{\mathrm{u}}(4) \gamma^{\nu}\left(1-\gamma^{5}\right) \mathrm{u}(2)\right)
$$


Taking the case where $\mathrm{q}^{2} \ll \mathrm{M}_{\mathrm{W}}^{2}$, averaging over initial spins, and summing over final spins, one finds

$$
\begin{aligned}
<|\mathcal{M}|^{2}>=\frac{1}{2} \sum_{\text {spins }}|\mathcal{M}|^{2}=\left(\frac{\mathrm{g}_{\mathrm{W}}^{2}}{8 \mathrm{M}_{\mathrm{W}}^{2}}\right)^{2} & \operatorname{Tr}\left[\gamma^{\mu}\left(1-\gamma^{5}\right)\left(\not p_{1}+\mathrm{m}_{\mathrm{e}}\right) \gamma^{\nu}\left(1-\gamma^{5}\right) p_{3}\right] \\
& \times \operatorname{Tr}\left[\gamma_{\mu}\left(1-\gamma^{5}\right)\left(\not p_{2}+\mathrm{m}_{\mathrm{e}}\right) \gamma_{\nu}\left(1-\gamma^{5}\right) p p_{4}\right]
\end{aligned}
$$

Using trace theorems, Equation 1.7 reduces to

$$
<|\mathcal{M}|^{2}>=2\left(\frac{g_{\mathrm{W}}}{\mathrm{M}_{\mathrm{W}}}\right)^{4}\left(\mathrm{p}_{1} \cdot \mathrm{p}_{2}\right)\left(\mathrm{p}_{3} \cdot \mathrm{p}_{4}\right)
$$

Using Equations 1.5 and 1.8, going to the center of mass frame, and considering relativistic energies where $\left(\frac{\mathrm{m}_{\mathrm{e}}}{\mathrm{E}_{\nu}}\right)$ is small, one can find

$$
\frac{\mathrm{d} \sigma}{\mathrm{d} \Omega}=\frac{1}{2}\left(\frac{\mathrm{g}_{\mathrm{W}}^{2} \mathrm{E}_{\nu}^{*}}{4 \pi \mathrm{M}_{\mathrm{W}}^{2}}\right)\left(1-\left(\frac{\mathrm{m}_{\mu}}{2 \mathrm{E}_{\nu}^{*}}\right)^{2}\right)^{2}
$$

where neglecting the mass of the electron, $\mathrm{E}_{\nu}^{*}=\frac{\mathrm{E}_{\nu}}{2}$ with $\mathrm{E}_{\nu}$ the energy of the incident muon neutrino assuming the electron has negligible momentum.

For a neutrino scattering off of a proton or neutron, there is no entirely analytical way to find the matrix amplitude $\mathcal{M}$. This is due to the nature of QCD and the structure of a nucleon. Aside from the three valence quarks within a nucleon, there are copius amounts of gluons as well as ephemeral sea quarks that arise from the gluons. Since the matrix element of interest is a second rank tensor, a solution to this problem is to assemble all possible second rank tensors that can contribute in the calculation of the cross-section and to find the form factors for each tensor component. These terms then capture the internal structure of the nucleon. An 


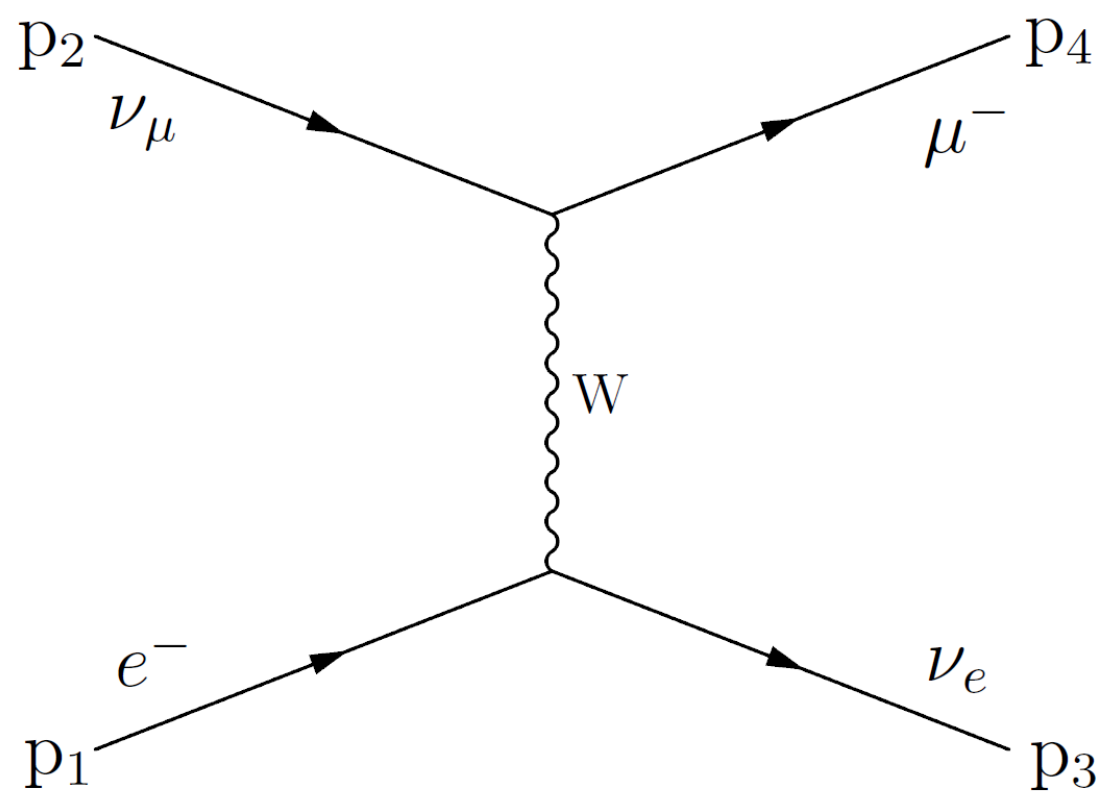

Figure 1.2: Feynman diagram of $\nu_{\mu}+\mathrm{e}^{-} \rightarrow \mu^{-}+\nu_{\mathrm{e}}$.

example of such a formalism is [1]

$$
\begin{array}{r}
\mathrm{W}^{\mu \nu}=-\mathrm{W}_{1} \mathrm{~g}^{\mu \nu}+\frac{\mathrm{W}_{2}}{\mathrm{M}_{\mathrm{p}}^{2}} \mathrm{p}^{\mu} \mathrm{p}^{\nu}+\frac{\mathrm{W}_{3}}{\mathrm{M}_{\mathrm{p}}^{2}}\left(\mathrm{p}^{\mu} \mathrm{q}^{\nu}-\mathrm{q}^{\mu} \mathrm{p}^{\nu}\right) \\
+\frac{\mathrm{W}_{4}}{\mathrm{M}_{\mathrm{p}}^{2}} \mathrm{q}^{\mu} \mathrm{q}^{\nu}+\frac{\mathrm{W}_{5}}{\mathrm{M}_{\mathrm{p}}^{2}}\left(\mathrm{p}^{\mu} \mathrm{q}^{\nu}+\mathrm{q}^{\mu} \mathrm{p}^{\nu}\right)
\end{array}
$$

where $\mathrm{M}_{\mathrm{p}}$ is the mass of the proton and the terms $\mathrm{W}_{1}, \mathrm{~W}_{2}, \mathrm{~W}_{3}, \mathrm{~W}_{4}$, and $\mathrm{W}_{5}$ are form factors. In this formalism, the anti-symmetric $\left(\mathrm{p}^{\mu} \mathrm{q}^{\nu}-\mathrm{q}^{\mu} \mathrm{p}^{\nu}\right)$ tensor does not contribute to the cross-section due to the Pauli exclusion principle. It is left to experiments to find the remaining form factors, which need not be constants. This is the general strategy employed to calculate cross-sections in interactions between neutrinos and hadrons. 


\subsubsection{Charged Current Quasi-Elastic Scattering}

CCQE scattering, an example of which is shown in Figure 1.3, is a process of the form $\nu_{l}\left(\bar{\nu}_{l}\right)+\mathrm{n}(\mathrm{p}) \rightarrow l^{-}\left(l^{+}\right)+\mathrm{p}(\mathrm{n})$. Here $l$ is a flavor of charged lepton, e, $\mu$, or $\tau$. This process is of particular importance to the neutrino oscillation community. In addition to being a significant component of the total cross-section for neutrinos with energies near $1 \mathrm{GeV}$, an estimate of the incoming neutrino energy can be made for CCQE interactions using the final state lepton kinematics. For CCQE scattering on a free nucleon, the differential cross-section $\frac{\mathrm{d} \sigma}{\mathrm{dQ}^{2}}$ can be found by the formalism outlined by C.H. Llewellyn Smith [34] which considers the different terms like in Equation 1.10 to derive

$$
\frac{\mathrm{d} \sigma}{\mathrm{dQ}^{2}}=\frac{\mathrm{M}^{2} \mathrm{G}^{2} \cos ^{2} \theta_{\mathrm{c}}}{8 \pi \mathrm{E}_{\nu}^{2}}\left(\mathrm{~A}\left(\mathrm{Q}^{2}\right) \mp \mathrm{B}\left(\mathrm{Q}^{2}\right) \frac{(\mathrm{s}-\mathrm{u})}{\mathrm{M}^{2}}+\frac{\mathrm{C}\left(\mathrm{Q}^{2}\right)(\mathrm{s}-\mathrm{u})^{2}}{\mathrm{M}^{4}}\right)
$$

where the term $\mp$ is negative for neutrinos and positive for anti-neutrinos, $G$ is the weak couping constant, $\mathrm{M}$ is the mass of the nucleon that the neutrino is scattering from, $\mathrm{E}_{\nu}$ is the incident (anti-)neutrino energy, $\theta_{\mathrm{c}}$ is the Cabibbo angle, $(\mathrm{s}-\mathrm{u})=$ $4 \mathrm{ME}_{\nu}-\mathrm{Q}^{2}-\mathrm{m}_{1}^{2}$, where $\mathrm{s}$ and $\mathrm{u}$ are Mandelstam variables[1], and $\mathrm{Q}^{2}$ is the square of the momentum transferred from the neutrino to the nucleon. The terms $\mathrm{A}\left(\mathrm{Q}^{2}\right)$, $\mathrm{B}\left(\mathrm{Q}^{2}\right)$, and $\mathrm{C}\left(\mathrm{Q}^{2}\right)$ are given by Equations $1.12,1.13$, and 1.14 as functions of vector form factors $F_{V}^{1}$ and $F_{V}^{2}$, pseudo-scalar form factor $F_{P}$, axial vector form factor $F_{A}$, and $\xi$ which is the difference between the anomalous magnetic moment of the proton and neutron. We use $\mathrm{Q}^{2}$ where $\mathrm{Q}^{2}=-\mathrm{q}^{2}$.

$$
\begin{array}{r}
\mathrm{A}\left(\mathrm{Q}^{2}\right)=\frac{\left(\mathrm{m}_{1}^{2}+\mathrm{Q}^{2}\right)}{\mathrm{M}^{2}}\left[(1+\tau)\left|\mathrm{F}_{\mathrm{A}}\right|^{2}-(1-\tau)\left|\mathrm{F}_{\mathrm{V}}^{1}\right|^{2}+\tau(1-\tau)\left|\mathrm{F}_{\mathrm{V}}^{2}\right|^{2}\right. \\
\left.+4 \tau \operatorname{ReF}_{\mathrm{V}}^{1 *} \xi \mathrm{F}_{\mathrm{V}}^{2}-\frac{\mathrm{m}_{1}^{2}}{4 \mathrm{M}^{2}}\left(\left|\mathrm{~F}_{\mathrm{V}}^{1}+\xi \mathrm{F}_{\mathrm{V}}^{2}\right|^{2}+\left|\mathrm{F}_{\mathrm{A}}+2 \mathrm{~F}_{\mathrm{P}}\right|^{2}-4(1+\tau)\left|\mathrm{F}_{\mathrm{P}}\right|^{2}\right)\right]
\end{array}
$$




$$
\begin{gathered}
\mathrm{B}\left(\mathrm{Q}^{2}\right)=\frac{\mathrm{Q}^{2}}{\mathrm{M}^{2}} \operatorname{ReF}_{\mathrm{A}}^{*}\left(\mathrm{~F}_{\mathrm{V}}^{1}+\xi \mathrm{F}_{\mathrm{V}}^{2}\right) . \\
\mathrm{C}\left(\mathrm{Q}^{2}\right)=\frac{1}{4}\left(\left|\mathrm{~F}_{\mathrm{A}}\right|^{2}+\left|\mathrm{F}_{\mathrm{V}}^{1}\right|^{2}+\tau\left|\xi \mathrm{F}_{\mathrm{V}}^{2}\right|^{2}\right) .
\end{gathered}
$$

We use as a shorthand the term $\tau=\frac{\mathrm{Q}^{2}}{4 \mathrm{M}^{2}}$. Note that the Llewellyn Smith derivation includes two terms $\mathrm{F}_{\mathrm{V}}^{3}$ and $\mathrm{F}_{\mathrm{A}}^{3}$ which are second class currents which are known to be either small or possibly non-existent [35] and would not contribute significantly to the cross-section if they were non-zero [36]. We therefore neglect form factors for second class currents in Equations 1.11, 1.12, and 1.13. Knowledge of the other form factors comes from several sources which we discuss in Section 1.3.2, 1.3.3, and 1.3.5.

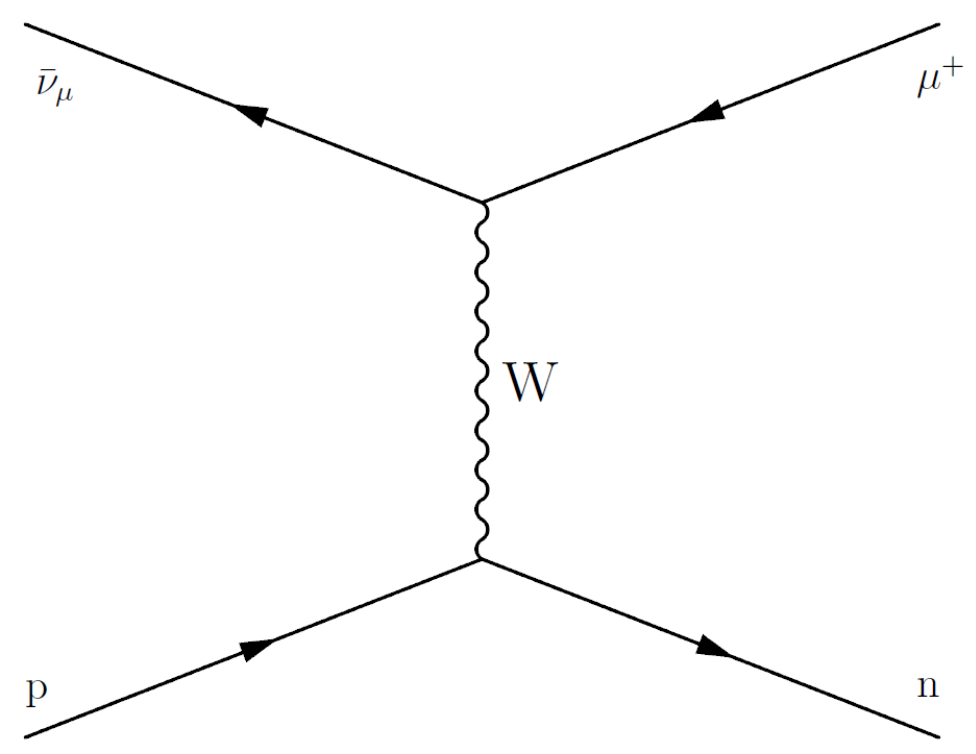

Figure 1.3: A Feynman diagram of muon anti-neutrino $\operatorname{CCQE}\left(\bar{\nu}_{\mu}+\mathrm{p} \rightarrow \mu^{+}+\mathrm{n}\right)$ scattering. 


\subsubsection{Vector Form Factors}

The Conserved Vector Current (CVC) hypothesis (discussed in Section 1.3.4) allows the vector form factors found in electron-proton and electron-deuteron scattering to be used in calculations for neutrino scattering. The values of the vector form factors at $\mathrm{Q}^{2}=0$ are known from the electric charges and magnetic moments of the nucleons. The remaining behavior of the form factors across $\mathrm{Q}^{2}$ is found using electron scattering data. Vector form factors for neutrino scattering are given in terms of the electric and magnetic form factors, $\mathrm{G}_{\mathrm{E}}^{\mathrm{V}}$ and $\mathrm{G}_{\mathrm{M}}^{\mathrm{V}}$, repectively, in Equations 1.15 and $1.16[37]$.

$$
\begin{aligned}
& \mathrm{F}_{\mathrm{V}}^{1}\left(\mathrm{q}^{2}\right)=\frac{\mathrm{G}_{\mathrm{E}}^{\mathrm{V}}\left(\mathrm{q}^{2}\right)-\tau \mathrm{G}_{\mathrm{M}}^{\mathrm{V}}\left(\mathrm{q}^{2}\right)}{1-\tau} \\
& \mathrm{F}_{\mathrm{V}}^{2}\left(\mathrm{q}^{2}\right)=\frac{\mathrm{G}_{\mathrm{M}}^{\mathrm{V}}\left(\mathrm{q}^{2}\right)-\mathrm{G}_{\mathrm{E}}^{\mathrm{V}}\left(\mathrm{q}^{2}\right)}{1-\tau}
\end{aligned}
$$

In Equations 1.15 and $1.16, \mathrm{M}$ is the nucleon mass, we use $\mathrm{q}^{2}$ instead of $\mathrm{Q}^{2}$, and $\tau$ $=\frac{\mathrm{Q}^{2}}{4 \mathrm{M}^{2}}$. The electric and magnetic form factors, $\mathrm{G}_{\mathrm{E}}^{\mathrm{V}}$ and $\mathrm{G}_{\mathrm{M}}^{\mathrm{V}}[37]$, are given by

$$
\mathrm{G}_{\mathrm{E}}^{\mathrm{V}}\left(\mathrm{q}^{2}\right)=\mathrm{G}_{\mathrm{E}}^{\mathrm{p}}\left(\mathrm{q}^{2}\right)-\mathrm{G}_{\mathrm{E}}^{\mathrm{n}}\left(\mathrm{q}^{2}\right)
$$

and

$$
\mathrm{G}_{\mathrm{M}}^{\mathrm{V}}\left(\mathrm{q}^{2}\right)=\mathrm{G}_{\mathrm{M}}^{\mathrm{p}}\left(\mathrm{q}^{2}\right)-\mathrm{G}_{\mathrm{M}}^{\mathrm{n}}\left(\mathrm{q}^{2}\right)
$$

It is the values of $G_{E}^{p}\left(q^{2}\right), G_{E}^{n}\left(q^{2}\right), G_{M}^{p}\left(q^{2}\right)$, and $G_{M}^{n}\left(q^{2}\right)$ that are extracted from electron scattering data. A fit to scattering data is done using a superposition of 
LaGrange polynomials and the Kelly form factor [38], the result of which we refer to as BBBA07 form factors [39]. The Kelly form factor [38] is given by

$$
\mathrm{G}\left(\mathrm{Q}^{2}\right) \propto \frac{\sum_{\mathrm{k}=0}^{\mathrm{n}} \mathrm{a}_{\mathrm{k}} \tau^{\mathrm{k}}}{1+\sum_{\mathrm{k}=1}^{\mathrm{n}+2} \mathrm{~b}_{\mathrm{k}} \tau^{\mathrm{k}}},
$$

where $\tau$ is defined above and $\mathrm{a}_{\mathrm{k}}$ and $\mathrm{b}_{\mathrm{k}}$ are found in a fit. At low $\mathrm{Q}^{2}$, the Kelly form factor and consequently the BBBA07 form factors mimic a dipole form, which has a physical interpretation of a non-point-like spatial distribution of the nucleon [39]. The dipole behavior occurs because close to zero $\mathrm{Q}^{2}$, the other higher order terms are negligible provided the coefficients are not particularly large in magnitude.

\subsubsection{Pseudo-Scalar Form Factor}

The pseudo-scalar form factor $\mathrm{F}_{\mathrm{P}}$ has been related to higher order corrections involving pions. The Partially Conserved Axial Current (PCAC) hypothesis, which predicts that the weak axial current is nearly conserved in nuclear interactions [33], allows $\mathrm{F}_{\mathrm{P}}$ to ultimately be related to the axial form factor, $\mathrm{F}_{\mathrm{A}}$. Using the GoldbergerTreiman relation [40], and following a derivation [36] based on PCAC, the pseudoscalar form factor is given by

$$
\mathrm{F}_{\mathrm{P}}=\frac{2 \mathrm{M}^{2} \mathrm{~F}_{\mathrm{A}}\left(\mathrm{Q}^{2}\right)}{\mathrm{Q}^{2}+\mathrm{m}_{\pi}^{2}},
$$

where $\mathrm{m}_{\pi}$ is the charged pion mass, $\mathrm{M}$ is the mass of the nucleon, and $\mathrm{F}_{\mathrm{A}}\left(\mathrm{Q}^{2}\right)$ is the axial form factor. Small deviations of Equation 1.20 from data have been found at higher values of $\mathrm{Q}^{2}[36]$. 


\subsubsection{Conserved Vector Current Hypothesis}

The CVC hypothesis posits that the vector component of the weak current in neutrino-nucleus scattering is the same as the vector component in charged leptonnucleon scattering. The justification for this is that an isospin triplet can be formed out of the weak current, the complex conjugate of that weak current, and the electromagnetic current which together should be be conserved by strong interactions [1]. This hypothesis has been found to hold experimentally, such as in the case of beta decay of ${ }^{14} \mathrm{O} \rightarrow{ }^{14} \mathrm{~N}$ [33].

\subsubsection{Axial Form Factor}

The axial form factor, $\mathrm{F}_{\mathrm{A}}\left(\mathrm{Q}^{2}\right)$, describes the axial structure of a nucleon. Based on experiments that we will discuss shortly, a dipole parameterization is often used, specifically,

$$
\mathrm{F}_{\mathrm{A}}\left(\mathrm{Q}^{2}\right)=\frac{\mathrm{g}_{\mathrm{A}}}{\left(1+\frac{\mathrm{Q}^{2}}{\mathrm{M}_{\mathrm{A}}^{2}}\right)^{2}},
$$

where the value $g_{A}=1.257 \pm 0.003$ is found in beta decay experiments [5] and $\mathrm{M}_{\mathrm{A}}$ is referred to as the axial mass. In this model, the term $\mathrm{M}_{\mathrm{A}}$ is the single free parameter.

The value of the axial form factor and $\mathrm{M}_{\mathrm{A}}$ can be found in several ways. Measurements of neutron beta decay give a precise value of this form factor at $\mathrm{Q}^{2}=0$ $\mathrm{GeV}^{2}$ [41]. Electron-nucleon scattering data can also be used, specifically, charged pion electroproduction data at the pion production threshold [41, 42]. This method uses the Rosenbluth technique [43] to find $\mathrm{M}_{\mathrm{A}}$ using several values of $\mathrm{Q}^{2}$, but has limitations. It is less precise than the alternative method for extracting the axial form factor, neutrino-nucleus scattering. It also uses only low values of $\mathrm{Q}^{2}$, less than 


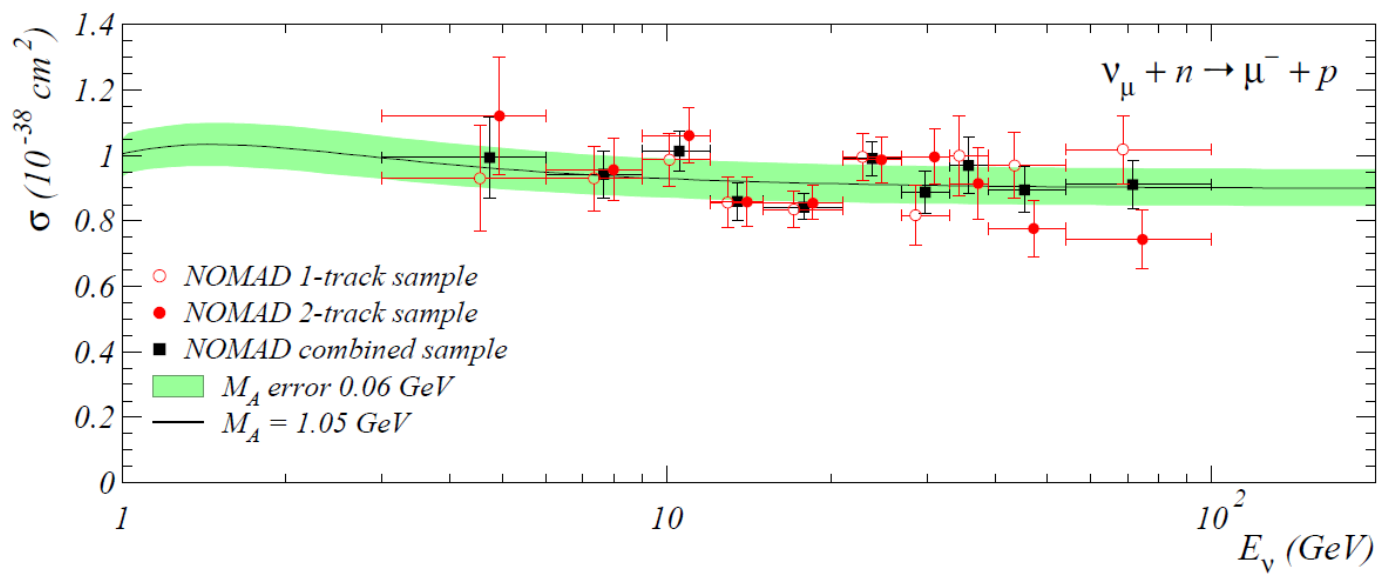

Figure 1.4: NOMAD CCQE cross-section data with comparison to simulation with $\mathrm{M}_{\mathrm{A}}$ $=1.05 \mathrm{GeV}$. Figure from [45].

$0.3 \mathrm{GeV}^{2}[41,42]$. Overall, electron scattering cannot be used to measure this form factor across $\mathrm{Q}^{2}$ since the electromagnetic force is substantially stronger than the weak force, meaning the axial form factor cannot practically be measured in that scenario.

The most precise method for finding the axial form factor is performing fits to CCQE neutrino-nucleon scattering data. Most fits use the formalism used in Equations 1.11, 1.12, 1.13, and 1.14. Results for the value of $\mathrm{M}_{\mathrm{A}}$ vary. A fit to deterium data finds a value of $\mathrm{M}_{\mathrm{A}}=1.014 \pm 0.014 \mathrm{GeV}$ [44]. The NOMAD experiment found a value of $\mathrm{M}_{\mathrm{A}}=1.05 \pm 0.02$ (stat) \pm 0.06 (syst) on neutrinos ranging from $3-100 \mathrm{GeV}$, as shown in Figure 1.4. This is similar to other measurements of $\mathrm{M}_{\mathrm{A}}$ that have been made previously [45]. The MiniBooNE experiment extracted $\mathrm{M}_{\mathrm{A}}=1.35 \pm 0.17 \mathrm{GeV}$. A comparison of MiniBooNE data to several values of $\mathrm{M}_{\mathrm{A}}$ is given in Figure 1.5. The MiniBooNE measurement of $\mathrm{M}_{\mathrm{A}}$ is substantially higher than other results and requires an enhanced Pauli blocking term to fit the data [46].

A higher value of $\mathrm{M}_{\mathrm{A}}$ leads to a higher predicted cross-section for CCQE scat- 


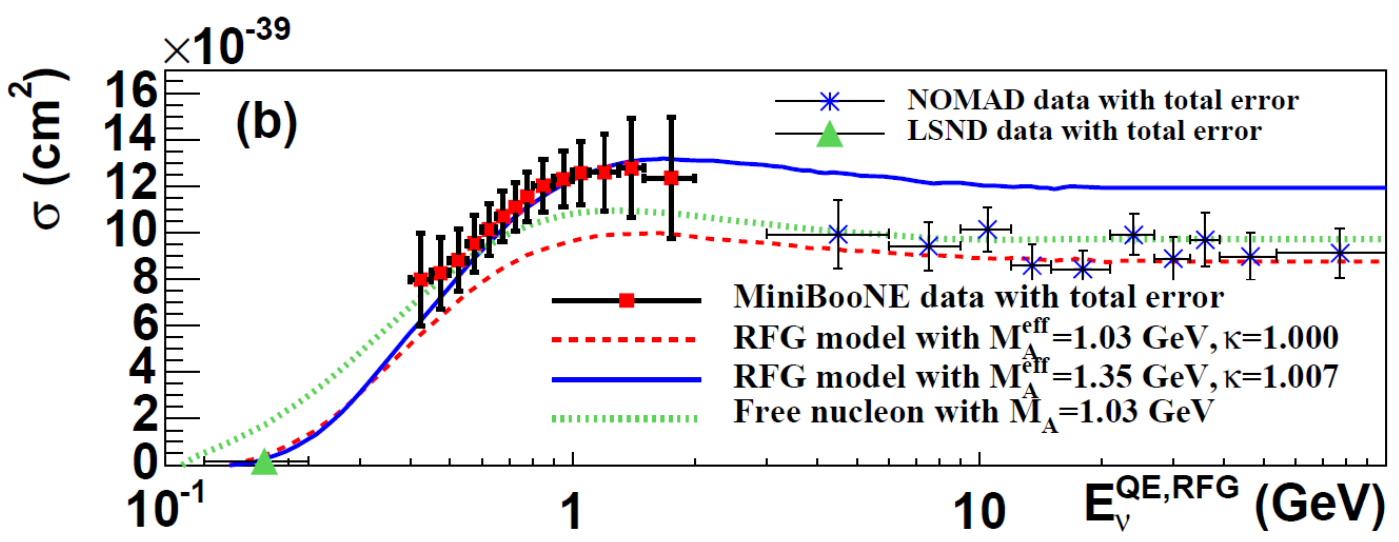

Figure 1.5: MiniBooNE CCQE cross-section data with comparison to simulation with $\mathrm{M}_{\mathrm{A}}$ of $1.03 \mathrm{GeV}$ and $1.35 \mathrm{GeV}$. Figure from [47].

tering, which is not consistent with other cross-section results. Much effort has gone into reconciling MiniBooNE results with past experiments. One suggested mechanism for the enhancement that MiniBooNE finds is meson exchange currents (MEC), discussed in Section 1.3.6. MINER $\nu \mathrm{A}$ will help resolve this issue with its good final state particle resolution and may also be sensitive to deviations from the dipole form of the axial form factor.

\subsubsection{Nuclear Effects}

The calculation of the cross-section in Equation 1.11 for CCQE scattering is for a free nucleon. Unless the scattering is on hydrogen, that nucleon is contained within a nucleus. The presence of the target nucleon in a nucleus can have several effects such as Pauli blocking, final state interactions, and other potential nuclear effects such as MEC. These can impact the predicted cross-section along with the final state kinematics.

In the case of Pauli blocking, an effect relating to the Pauli exclusion principle 
is active. The nucleons within the nucleus have a distribution of momenta which corresponds to different quantum mechanical states. An interaction will be suppressed in cases where a final state nucleon would be imparted energy that would push it into an already occupied state. This imparts a threshold for scattering based on the energy transfer from the neutrino to the final state hadron, and affects the cross-section for a particular process.

Final state interactions (FSI) involving the final state hadron and the nucleus the interaction is occuring in can also have large effects. This can involve scattering of the final state hadron as it exits the nucleus. This modifies the angle and energy of that final state particle. Something more dramatic can happen, in which FSI can create pions or eject other nucleons. A particular interaction may be quasielastic, but may have particles that are not consistent with CCQE scattering such as pions. This can lead to the loss of signal events if this is not simulated correctly. The converse can happen as well, where a non-CCQE interaction can appear as signal due to FSI. Likely the most common example of this is pion absorption in the nucleus. In this case, the only observable final state particles will be a muon and a nucleon, which will likely appear as a CCQE interaction.

Other nuclear effects may also be at work such as short range correlations [48] and MEC [49]. These effects can modify scattering cross-sections. Short range correlations affect the momentum distribution of a target nucleon, which can counteract Pauli blocking and modify the final state kinematics. Short range correlations can lead to momenta higher than would be predicted by a Relativistic Fermi Gas (RFG) Model [50], which many simulations employ. Since reconstruction of an event often assumes a nucleon is at rest, short range correlations also can cause reconstruction errors or smearing beyond what would be predicted by the RFG. The topics of RFG 
and MEC are discussed below.

\section{The Relativistic Fermi Gas Model}

A commonly used model of the nucleus is the RFG model. This model involves treating nucleons within a nucleus as a non-interacting Fermi gas. For a Fermi gas in the ground state, particles occupy energy levels ranging from the lowest possible energy up to the Fermi energy. In the RFG model of a nucleus, we consider the maximum momentum, known as the Fermi momentum.

The common way to implement this model in a simulation is a step function where if a final state nucleon's momentum is below the Fermi momentum, the function and consequently the cross-section is zero. A binding energy term, $\mathrm{E}_{\mathrm{b}}$, is also considered. This is the amount of energy required to separate a nucleon from a particular nucleus and affects the final state energy of a nucleon [50].

This model has limitations. Perhaps the largest issue is the assumption that nucleons are non-interacting, which we know to be false. Short range correlations can lead to nucleons having a momentum greater than the Fermi momentum. To accomodate this, some models contain a high side tail for nucleon momentum [51]. Effects such as MEC, which involve the exchange of mesons between nucleons, are suspected to be present and could lead to a higher than otherwise predicted crosssection. Other models of the nucleon behavior within the nucleus do exist, such as spectral functions.

\section{Spectral Functions}

Spectral functions describe the probability of removing a proton or neutron with a particular momentum from a nucleus. A spectral function is assembled by consid- 
ering nucleons in two different scenarios. The first case involves filling states using the shell model of the nucleus. Most nucleons within a nucleus occupy such a state. The wave functions for nucleons up to the number that are predicted to be in such a state are used to directly calculate this particular component of a spectral function. This component of the spectral function can be modeled as a gaussian distribution, the details of which depend upon the nucleus. A small fraction of nucleons are in a higher energy state due to short range correlations. This component of the structure function is found using the wavefunctions of the correlated pair and maximum and minimum momenta of that pair $[52,48]$. This model can be used as an alternative to the RFG model.

\section{Meson Exchange Currents}

MEC is one proposal to explain the enhancement observed in CCQE scattering in certain experiments. An abundance of diagrams can contribute to the MEC process, but in general, a neutrino scatters off of a nucleon undergoing meson exchange with another nucleon. The final states in MEC are fundamentally different from CCQE scattering, but since final state nucleons are not necessarily observed, the process can appear as a CCQE event. For processes that have identical final states, interference can occur between diagrams when calculating a cross-section [1]. That is not applicable in the case of CCQE scattering and MEC. If MEC is present, it would enhance the overall charged current cross-section. Since MiniBooNE is not sensitive to final state nucleons, this has been put forth as a possible explanation for the size of the MiniBooNE CCQE cross-section [49].

Many different models are used to account for the effect of MEC or other similar processes that suspected to occur. One model captures the effect of MEC as an en- 
hancement of the magnetic form factor of the nucleon [53]. This method is attractive due to the simplicity of implementing it, but it lacks details of the final state. Many other more sophisticated models exist $[54,55,56,57,58,59,60]$. Additional data are needed to verify that MEC or similar processes are present in neutrino-nucleus interactions and to test the various models that now exist. 


\section{Chapter 2}

\section{The Accelerator Complex}

The neutrinos and anti-neutrinos that MINER $\nu \mathrm{A}$ measures come from the Neutrino Beam for the Main Injector (NuMI). NuMI is part of a larger complex at Fermi National Accelerator Laboratory (FNAL, Fermilab) that produces beams for a variety of physics experiments as depicted in Figure 2.1. To create a neutrino beam, a beam of protons is needed. We describe below the stages necessary to accelerate these protons as well as the eventual collisions that lead to the neutrinos and anti-neutrinos observed in MINER $\nu \mathrm{A}$.

\subsection{Accelerating Protons}

The first step in the accelerator chain is the ionization of diatomic hydrogen into $\mathrm{H}^{-}$ions. These $\mathrm{H}^{-}$ions are then accelerated by a Cockcroft-Walton to an energy of $750 \mathrm{keV}[61]$.

The $\mathrm{H}^{-}$ions are fed to a Linac (LINear ACcelerator) and accelerated to an energy of $116 \mathrm{MeV}$ using a series of drift tubes. A drift tube is an electrically-resonant 


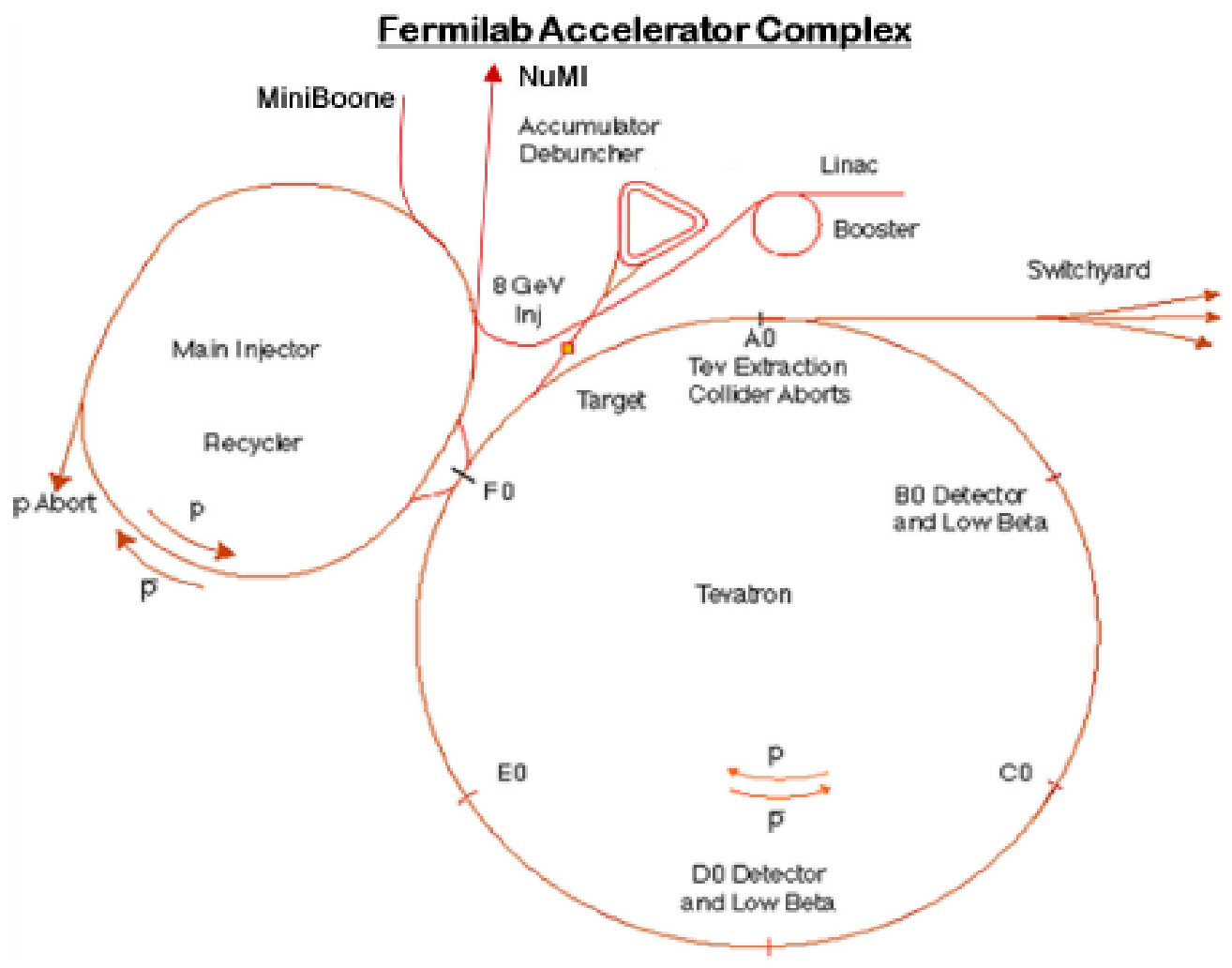

Figure 2.1: An overhead view of the various beam facilities at Fermi National Accelerator Laboratory. The Linac, Booster, and Main Injector accelerate protons which are consumed by the NuMI beam [62].

copper cylinder that creates an alternating electrical field. If injected during the appropriate phase, the $\mathrm{H}^{-}$ions will be accelerated in the forward direction. After traversing the drift tubes, the ions pass through Side-Coupled Linacs (SCL). SCLs are coupled cavities each powered by a 12 MW Klystron. The sequence of SCLs accelarates the $\mathrm{H}^{-}$ions to an energy of $401 \mathrm{MeV}$ [63].

The $\mathrm{H}^{-}$ions are transported to the Booster for injection. The Booster is a 150 meter diameter synchotron. The ions pass through a foil during injection stripping 
away the electrons and leaving a proton beam. Over multiple circulations within the Booster, protons are accelerated to an energy of $8 \mathrm{GeV}$ [64].

Once protons in the Booster reach $8 \mathrm{GeV}$ in energy, they are ready for transfer to the Main Injector. For the purposes of injecting beam in the Main Injector with minimal losses and damage, a particular batch structure is enforced within the Booster. This structure is visible within the NuMI beamline, as shown in Figure 2.2 [64].

The Main Injector is a synchotron which accelerates protons intended for the NuMI beamline to an energy of $120 \mathrm{GeV}$ [65]. Upon reaching $120 \mathrm{GeV}$ of energy, protons are ready to be transfered to the NuMI beamline. Proton spills can be transfered to the NuMI beamline every 2.06 or 2.20 seconds during this run. Bunches are generally about 10 microseconds in width, which varies slightly depending upon whether the Recycler Accelerator for the Tevatron is running.

\subsection{The NuMI Beamline}

The NuMI beamline can be configured to produce muon neutrinos or muon antineutrinos. Additionally, the energy spectrum of the resulting neutrinos and antineutrinos can be modified by altering certain components of the beam. For this analysis, the Main Injector delivered up to $35 \times 10^{12}$ protons on target $(\mathrm{POT})$ per spill, which is close to the design maximum of $40 \times 10^{12}$ POT. Here, POT refers to the number of protons delivered from the Main Injector to the NuMI target. The terms upstream and downstream are defined by the direction of the NuMI beam. Upstream refers to something being closer to the source of the beam and downstream refers to something further from the source of the beam. A step by step description of this beamline follows. 


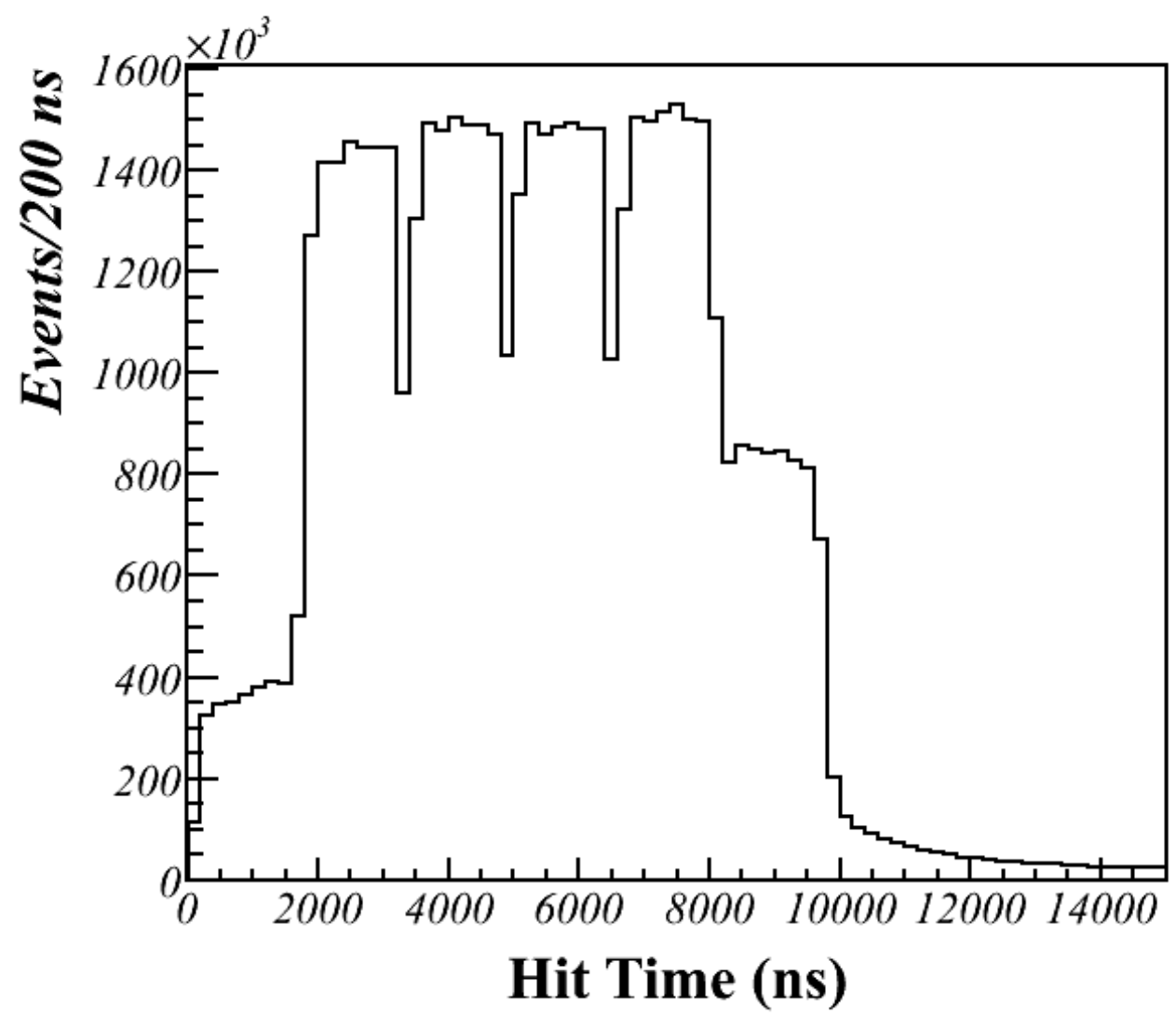

Figure 2.2: Distribution of MINER $\nu \mathrm{A}$ hits demonstrating bunch structure for the NuMI beamline. A portion of the tail in the plot is from particle decays within the detector.

Protons for the NuMI beamline are extracted from the Main Injector and directed downward at an angle of 58 milliradians. This angle was selected to facilitate MINOS [66], a long baseline neutrino oscillation experiment. This angle is accounted for at the analysis stage and has a minor impact on acceptance.

After protons are injected into the NuMI beam, they pass through a baffle that acts as a collimator before colliding with the NuMI target [67]. The NuMI target is water cooled and made of 47 segments of $2 \mathrm{~cm}$ long graphite (carbon) pieces with $0.3 \mathrm{~cm}$ spacing between each piece [68]. Together, this forms a target roughly two 
interaction lengths (approximately one meter) long, $15 \mathrm{~mm}$ in height, and $6.4 \mathrm{~mm}$ in width [69]. The proton beam is about $1 \mathrm{~mm}$ in diamter [70] when it collides with the NuMI target with a maximum divergence of 60 microradians [71]. Proton interactions with the nuclei of the target create pions and kaons which can subsequently reinteract within the target. The target diameter is thin to minimize this reinteraction. Reinteractions change the resulting spectrum of the pions and kaons. Modeling reinteractions is difficult and is consequently a large source of systematic error for our analysis which is discussed in Section 5.3.5.

The resulting spray of pions and kaons as well as any left over protons then travel toward the NuMI horns, which are depicted in Figure 2.3. The NuMI horns are two toroidal electromagnets with parabolic curvature. Together, the two horns act as a lens system. The focusing of the horn system can be modified by changing the current applied to the horns including reversing the polarity of that current. Changing the polarity flips the sign of the magnetic field and consequently changes which type of charge is focused or defocused. The magnitude of the current changes the size of the magnetic field within the horns which determines the focal point of the system. The focal point is also dependent upon the momentum of the pion or kaon.

Pions and kaons with too low of momentum will not be sufficiently focused to contribute to the flux. Since very high energy pions and kaons are over-focused if they pass through the horns, these too will not contribute to the flux. However, some pions and kaons can pass through an aperature in the center of the horns, which we refer to as the "neck." This includes very high energy pions and kaons which consequently contribute to a high energy tail in the neutrino and anti-neutrino energy spectrum. The particular spectrum of pions and kaons focused by the horns can 


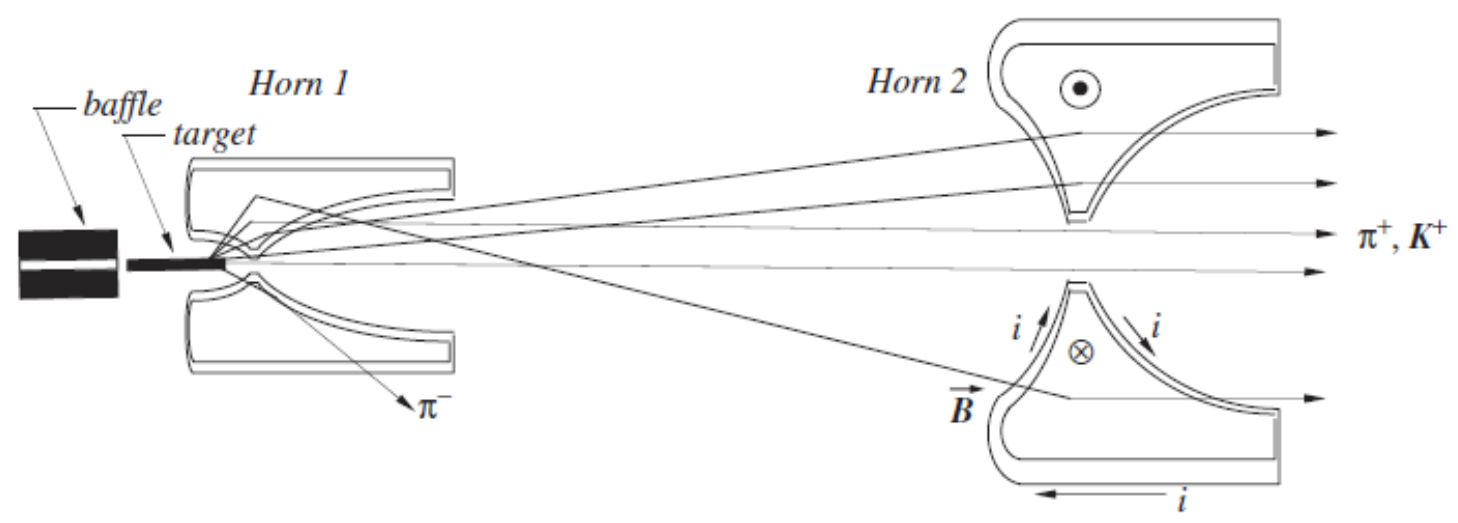

Figure 2.3: A schematic of the two magnetized parabolic NuMI horns used to focus the pions and kaons prior to their decay. Distances in the figure are for illustrative purposes. [68].

also be modified by changing the spacing between the target and the horn system. Last, the spacing between the two horns can be adjusted although in practice this is never varied. If no current is passing through the horns, no focusing occurs [68]. An illustration of pions and kaons passing through the horn system is in Figure 2.3.

For this analysis, a horn-target spacing of $10 \mathrm{~cm}$ and horn current of $-185 \mathrm{kA}$ was used. The $10 \mathrm{~cm}$ spacing corresponds to roughly $25 \mathrm{~cm}$ of the target lying within the horn system. We refer to this configuration as Reverse Horn Current (RHC) Low Energy (LE) running. Note that there are several configurations that are refered to as LE running. In typical Forward Horn Current (FHC) LE running, accounting for cross-sections, we expect roughly $91.7 \% \nu_{\mu}, 7.0 \% \bar{\nu}_{\mu}$, and $1.3 \% \nu_{e}$ and $\bar{\nu}_{e}$ [21]. The RHC mode, which is nominally an anti-neutrino beam, has siginificant amounts of neutrinos and anti-neutrinos. In the RHC beam, again accounting for cross-sections, we expect $58.1 \% \nu_{\mu}, 39.9 \% \bar{\nu}_{\mu}$, and $2.0 \% \nu_{e}$ and $\bar{\nu}_{e}[21]$. The muon neutrinos form a broad high energy tail for the overall RHC spectrum while the muon anti-neutrinos are peaked around an energy of a few $\mathrm{GeV}$.

After passing through the horn system, the pions and kaons, along with any 
protons left over from the original beam, enter a $675 \mathrm{~m}$ decay pipe, which is depicted in Figure 2.4. This pipe is 2 meters in diameter and has been evacuated and then filled with 13.2 PSI of helium. This pressure increases slightly during beam operation due to heating of the helium by particle interactions with the helium [72]. The presence of helium is to minimize pion absorption and pion interactions which would occur more frequently in the presence of air. Most pions and kaons decay to neutrinos and muons through the following decays modes: $\pi^{+} \rightarrow \mu^{+}+\nu_{\mu}$, $\mathrm{K}^{+} \rightarrow \mu^{+}+\nu_{\mu}, \pi^{-} \rightarrow \mu^{-}+\bar{\nu}_{\mu}$, and $\mathrm{K}^{-} \rightarrow \mu^{-}+\bar{\nu}_{\mu}$. Protons, undecayed pions and kaons, and muons pass through a Hadron Monitor at the end of the decay pipe. The Hadron Monitor is an array of small helium ionization chambers which measure the amount of ionization created by charged particles along with the spatial distribution of those particles. This detector is in place to monitor that the beam is functioning properly [73]. These particles then pass into the Hadron Absorber. The Hadron Absorber is a large mass of concrete blocks with enough material to fully stop most hadronic particles in the beam.

Since muons in the few GeV energy range are minimum ionizing particles, most pass through the Hadron Absorber without being stopped. Directly after the Hadron Abosrber is the first of three Muon Monitors. Figure 2.5 illustrates the location of each monitor. These monitors use the same ionization chambers as the Hadron Monitor, but are spread out over a larger area. This is to account for the divergence of the muons as they travel. The two other Muon Monitors are placed within alcoves drilled into the dolomite rock present at that depth. Each of the three Muon Monitors is beam centered. Since muons must pass through increasing amount of rock to reach the two successive Muon Monitors, the energy threshold for a muon increases for each successive monitor. The threshold allows for a measurement of the 


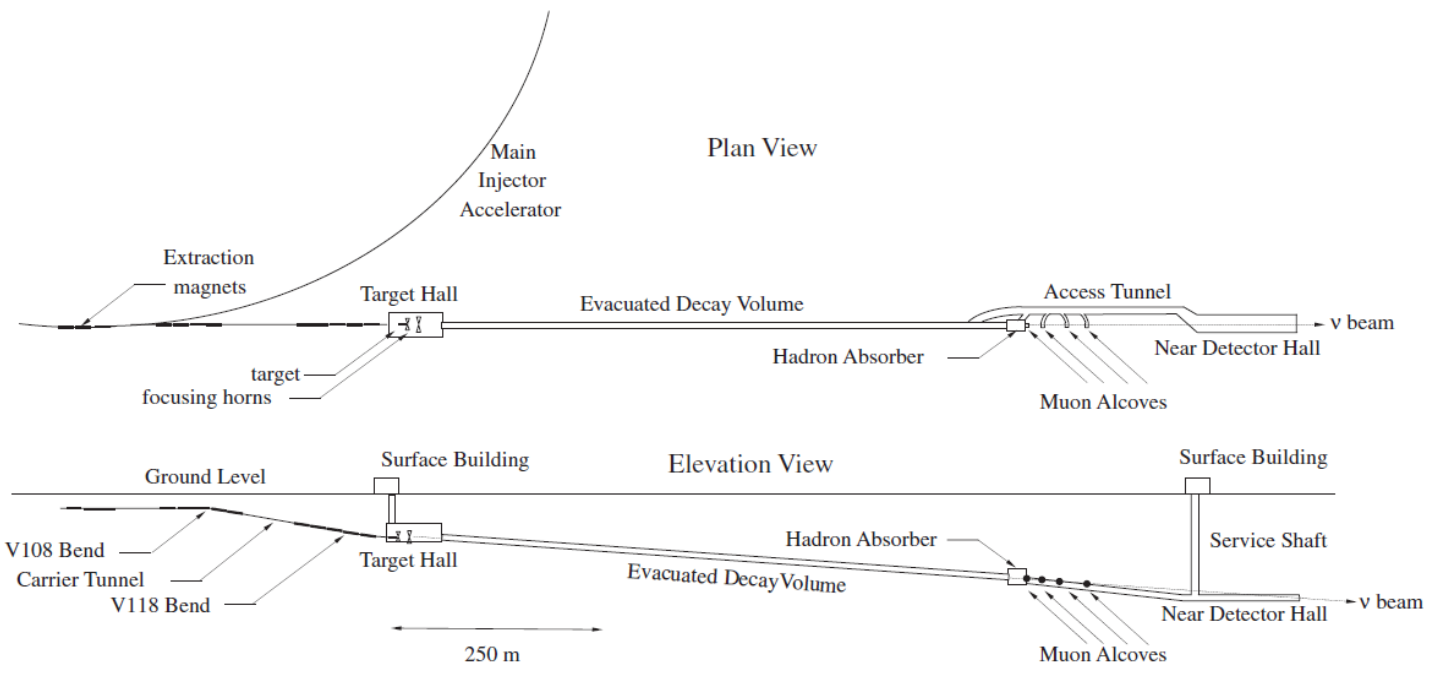

Figure 2.4: An overhead and cut-away view of the NuMI facility. Protons are extracted from the Main Injector and are directed toward a target producing pions and kaons. The pions and kaons are focused by a horn system after which they travel down a decay pipe. These pions and kaons decay overwhelmingly to muons and neutrinos. The Hadron Absorber stops pions, kaons, and protons. The muons and neutrinos then enter dolomite rock that extends for 240 meters. Muons range out within the rock and the neutrinos travel onward toward the Near Detector Hall. [68].

muon energy spectrum. This information is currently being used to constrain the prediction of the pion and kaon spectrum and consequently the expected neutrino and anti-neutrino energy spectrum. In total, 240 meters of dolomite separate the decay pipe and the Near Detector Hall. This amount of material is sufficient to range out all muons originating from the NuMI beamline. The neutrinos pass through the Hadron Absorber and rock unattenuated to the Near Detector Hall. 


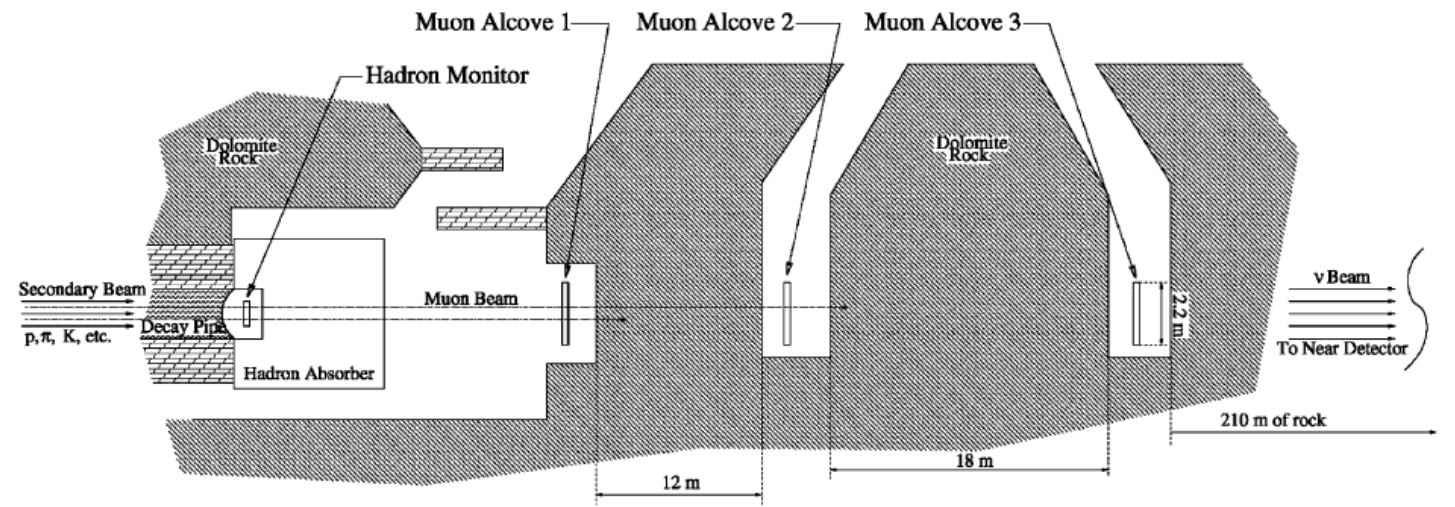

Figure 2.5: Locations of the Hadron Monitor and three Muon Monitors in the NuMI facility [73]. 


\section{Chapter 3}

\section{The MINER $\nu$ A Detector}

MINER $\nu \mathrm{A}$ is a finely segmented tracking and sampling detector. It contains an inner detector (ID) with a tracking region, downstream and side electromagnetic calorimeters (ECAL), and a downstream hadronic calorimeters (HCAL). An outer detector (OD) surrounds the ID. The MINOS Near Detector, which is downstream of the MINER $\nu \mathrm{A}$ detector, is used as a spectrometer for muons that exit the downstream end of MINER $\nu \mathrm{A}$. During the data taking period for this analysis, the ArgoNeuT detector was situated between the MINOS and MINER $\nu$ A detectors. The fully installed MINER $\nu \mathrm{A}$ detector also contains a variety of nuclear targets. These were not present at the time of the data taking for this analysis and are not dicussed further in this dissertation.

\subsection{The MINER $\nu$ A ID}

The ID is composed of the Tracker, ECAL, and HCAL regions, as depicted in Figure 3.1. Each region is composed of hexagonal modules which differ in composition 


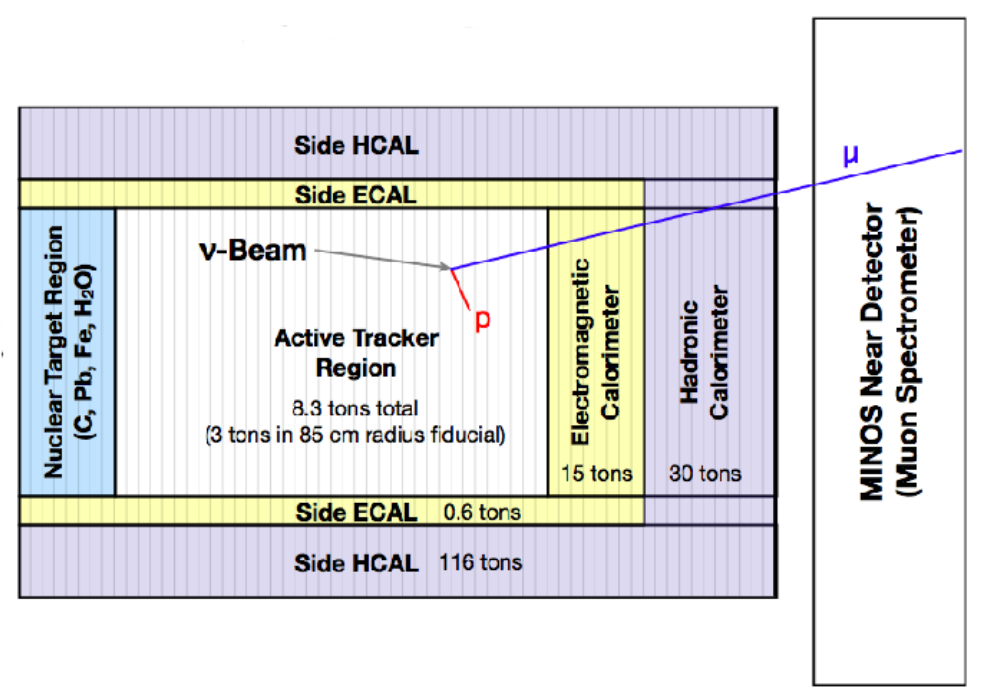

Figure 3.1: A cross-sectional view of the MINER $\nu$ A detector. For illustrative purposes, the direction of the beam and a cartoon example of a neutrino interaction where the final state muon passes into MINOS is also shown. The Frozen detector, the configuration on which data for this analysis were taken, does not contain nuclear targets and has only a subset of the total number of Tracker modules installed. ArgoNeuT is also not pictured.

according to region.

\subsubsection{The Tracker}

The Tracker region contains fully active modules, each of which is composed of two planes. A detailed description of a plane and its structure can be found in Section 3.5. A plane can have one of three different views, $\mathrm{X}, \mathrm{U}$, or $\mathrm{V}$. X planes have scintillator strips aligned vertically. Hits in this view give position information in the positive and negative $\mathrm{x}$-directions. The $\mathrm{U}$ and $\mathrm{V}$ planes are then rotated 60 degrees clockwise and counterclockwise from the X plane in the XY plane. Three different views are used since three dimensional reconstruction using two orthogonal planes can have ambiguity in certain reconstruction scenarios.

The coordinate system we use in MINER $\nu \mathrm{A}$ reconstruction is right-handed, but 


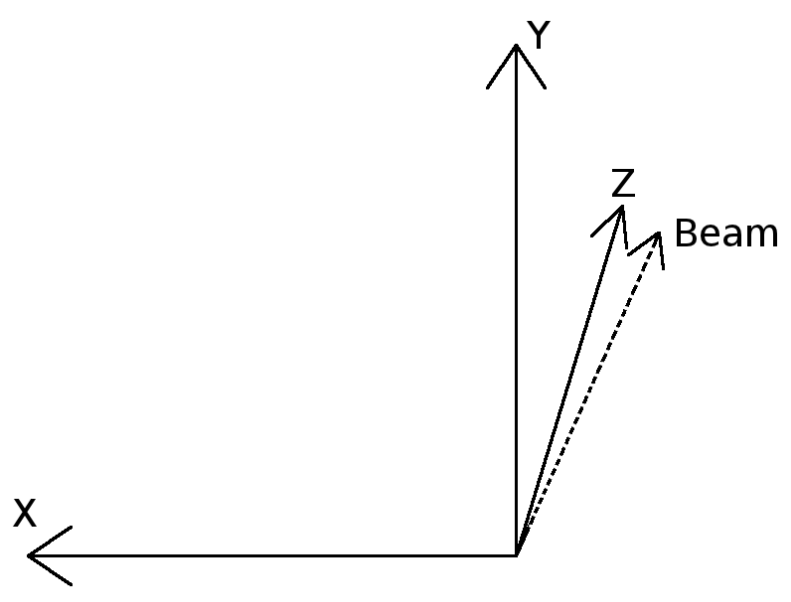

Figure 3.2: The schematic shows the MINER $\nu \mathrm{A}$ coordinate system. The $\mathrm{Y}$-axis is defined to be away from the center of the Earth and the Z-axis is defined to be in the general direction of the beam. This requires that the $\mathrm{X}$ direction be to the left to retain a right handed coordinate system. The direction of the beam, which is at an angle 58 milliradians in the negative $\mathrm{Y}$ direction with respect to the $\mathrm{Z}$ axis in the $\mathrm{YZ}$ plane, is depicted as well.

with an unusual orientation. Since the positive Z-direction is defined to be in the direction that neutrinos from NuMI travel and the positive Y-direction is defined to be up (away form the center of the Earth), this requires the positive X-direction to be to the left when looking in the positive $\mathrm{Z}$ direction. Although this orientation may appear unusual, it preserves the right-handedness of the coordinate system. See Figure 3.2 for a schematic depicting the MINER $\nu$ A coordinate system.

Each module in the Tracker region is then composed of either a $\mathrm{X}$ and $\mathrm{U}$ plane or a $\mathrm{X}$ and V plane. Modules are then UX or VX in structure, where the $\mathrm{X}$ plane is always on the downstream end of the module. The modules then alternate between UX and VX structure. In total, the Tracker region contained 34 modules for the "Frozen" data run, which is the data set used for this analysis. Figure 3.3 shows a cross-sectional view of a module.

Modules in this region also contain what we refer to as the side-ECAL. This 


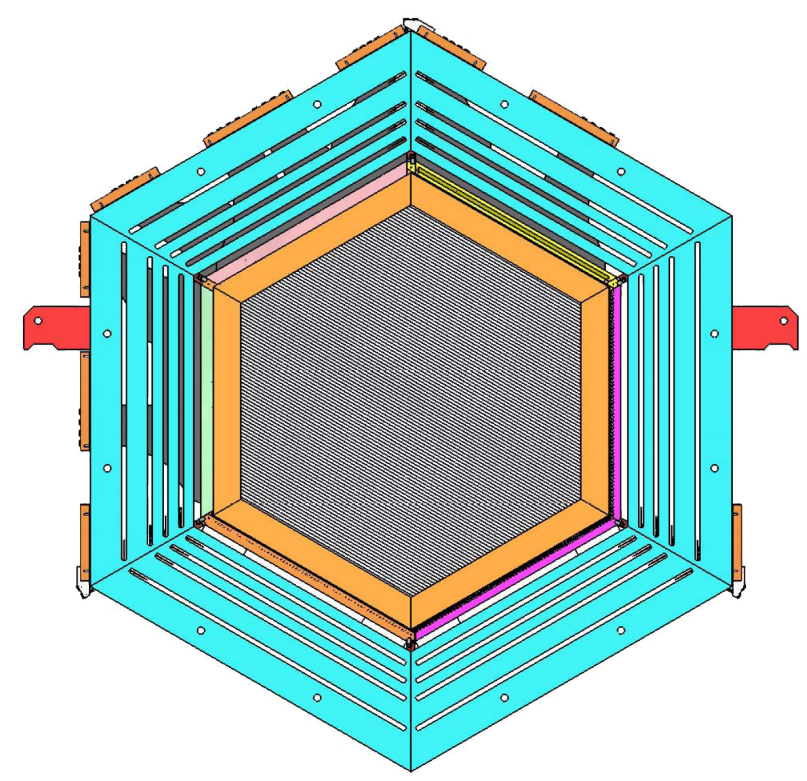

Figure 3.3: A cross-sectional schematic of a MINER $\nu \mathrm{A}$ module. Visible are the inner detector strips, lead collar, outer detector frames, and side clips that support the module.

is a lead collar that, starting at roughly $90 \mathrm{~cm}$ from the center of the module and extending to the outer edge, forms a hexagonal ring. Only Tracker modules contain a lead collar. The purpose of the lead collar is to prevent electromagnetic showers that originate in the tracker from escaping the ID.

A transition module exists between the last tracker module and first ECAL module. This module contains a $1 \mathrm{~mm}$ thick lead sheet on the downstream end of the last plane in the module so that each plane of the ECAL has a lead absorber upstream of it.

\subsubsection{The ECAL}

The ECAL modules are very similar to the Tracker modules, but with $1 \mathrm{~mm}$ lead sheets on the downstream edge of each plane in the module. Modules in the ECAL have an alternating UX and VX modules like in the Tracker region. In total, there 
are 10 modules in the ECAL. The ECAL causes photons and electrons to shower in a predictable way.

\subsubsection{The HCAL}

Modules in the HCAL have a fundamentally different structure from modules in the Tracker or the ECAL. Each module contains a 1 inch thick steel absorber and a scintillator plane downstream of the steel. The scintillator planes that compose the HCAL modules alternate in view, having a repeating pattern of XVXU. In total, the HCAL is made of 20 modules. The large mass of the HCAL generally helps contain particles originating from the tracker region in the ID with the exception of energetic muons.

\subsection{The MINER $\nu$ A OD}

The OD is composed of scintillator strips embedded in steel frames. These are visible as the outermost layer in Figure 3.3. The OD is present to range out particles that escape the sides of the detector. We do not use information from the outer detector for the analysis presented in this dissertation.

\subsection{Description of Frozen Detector}

Construction of MINER $\nu$ A was in progress when data collection for this analysis began. RHC running was projected to end just as construction of the detector was to be completed. At the time this was the only scheduled period of RHC running. To ensure that MINER $\nu \mathrm{A}$ could make cross-section measurements for 
anti-neutrinos, data collection began with a partially constructed detector. This configuration contained 34 active Tracker modules, 10 active ECAL modules, and 20 active HCAL modules. We refer to this configuration as the "Frozen Detector."

This running period can be divided into two periods. In the first period, after a sufficient number of modules had been installed, a 1 inch steel target referred to as the TARP was installed along with two additional modules just upstream and downstream from the TARP. All further construction activity was halted and data taking then began. This period where we halted construction is the origin of the term Frozen Detector. Approximately $4 \times 10^{19}$ POT worth of data were recorded in this configuration. In the second period, the TARP was removed and construction resumed, but no modules were added to the operational volume until the completion of $\mathrm{RHC}$ running. In this period, about $5 \times 10^{19}$ POT were delivered. We perform tests to ensure that no significant systematic differences exist between these two data sets. Note that the TARP is not used in this analysis.

\subsection{The Mirror Plane}

A steel plane is installed just downstream of the last HCAL planes. This plane spans only a portion of the detector in the horizontal direction, but provides complete coverage in the vertical direction. This plane is positioned to provide shielding for the HCAL from the magnetic field generated by the MINOS coil. 


\subsection{Plane Composition}

Although modules differ by detector region, all planes have the same basic structure regardless of view. Each plane contains 127 alternating triangular scintillator strips. These strips are arranged in a saw-tooth pattern shown in Figure 3.4. The alternating pattern of strips allows for very precise position measurements. A particle that traverses a plane will most likely deposit energy in two strips. The proportion deposited in each strip can be used to form an energy weighted position measurement. This configuration of strips yields a position resolutions of roughly $3 \mathrm{~mm}$.

Each plane is sealed by Lexan $\left(\mathrm{C}_{10} \mathrm{H}_{12} \mathrm{O}_{3}\right)$ and electrical tape (undisclosed by manufacturer, most likely PVC $\left.\left(\mathrm{C}_{2} \mathrm{H}_{3} \mathrm{Cl}\right)\right)$. This is to ensure that ambient light does not drown out any signals coming from a particle interaction. This is a source of passive material and is accounted for.

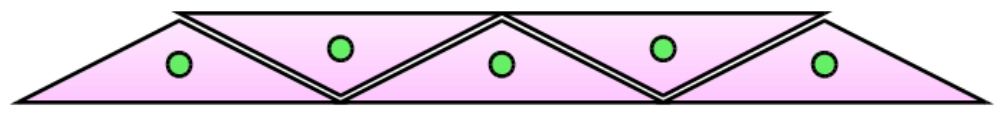

Figure 3.4: A diagram of the alternating MINER $\nu$ A Inner Detector scintillator strips that make up a plane.

\subsubsection{MINER $\nu$ A Strips}

MINER $\nu \mathrm{A}$ strips are triangular shaped scintillator bars of varying length. Strip lengths depend on a particular strip's position within a plane. Taking a crosssectional view, the strips are isosceles triangles $3.3 \mathrm{~cm}$ in width and $1.7 \mathrm{~cm}$ in height with the $3.3 \mathrm{~cm}$ base being the longest side of the triangle.

The scintillator is composed of Dow Styron $663(\mathrm{~W})$ polystyrene $\left(\left(\mathrm{C}_{8} \mathrm{H}_{8}\right)_{\mathrm{n}}\right)[74]$. Within this plastic are two dopants. One dopant, 2,5-diphenyloxazole $\left(\mathrm{C}_{15} \mathrm{H}_{11} \mathrm{NO}\right)$, 
or PPO for short, comprises roughly $1 \%$ of the scintillator mass. The other dopant, 1,4-bis(5-Phenyloxazole-2-yl) benzene $\left(\mathrm{C}_{24} \mathrm{H}_{16} \mathrm{~N}_{2} \mathrm{O}_{2}\right)$, known as POPOP, is present at about the $0.03 \%$ level. The two dopants convert energy from particle interactions within the scintillator to wavelengths of light that can be transmitted by the Wave Length Shifting (WLS) fiber [75].

Each strip contains a $2.6 \mathrm{~mm}$ diameter hole running the entire length of the strip. The hole is centered along the $3.3 \mathrm{~cm}$ base with the center of the hole lying $0.85 \mathrm{~cm}$ above the base. Each hole is filled with WLS optical fiber. The WLS fiber is held in place by optically clear epoxy.

A $0.25 \mathrm{~mm}$ layer of Capstock, composed of polystyrene and titanium dioxide $\left(\mathrm{TiO}_{2}\right)$, covers each strip. The $\mathrm{TiO}_{2}$ comprises $15 \%$ of the Capstock by mass. This material is applied during the coextrusion step during the manufacturing process of a strip. This material has high reflectivity; this is necessary because light can undergo multiple reflections within a strip before being captured by the WLS fiber.

\subsubsection{MINER $\nu$ A WLS and Clear Optical Fiber}

\section{WLS Fiber}

Each MINER $\nu$ A strip has a WLS $1.2 \mathrm{~mm}$ diameter, $175 \mathrm{ppm}$ (Y-11), S-35, multiclad fiber made by Kuraray. Multiclad refers to multiple layers of material around a central core of material within the fiber [76]. This design leads to lower attenuation as light travels along the fiber than if a single core were used [76]. The other specifications refer to the chemical composition of the fiber, which Kuraray does not disclose. WLS fiber is used to shift wavelengths of light from those created within a strip to a spectrum that is suitable for the Photomultiplier Tubes (PMT). 
The end of each fiber that is inserted into a strip has a diamond polish to ensure the end of the fiber is flat. A mirror finish is applied to the end of the fiber by vacuum sputtering of Aluminum. Since light can travel in either direction, toward or away from the PMT, having a mirror finish increases the total amount of light yield by reflecting light back toward the PMT tube.

The WLS fiber varies in length depending upon the lengths and position of a strip, but fibers are on average $2.7 \mathrm{~m}$ in length. Outside of a strip, the WLS fiber mates to a clear fiber optical cable. Both WLS and clear optical fibers are wrapped in a covering to shield from ambient light.

\section{Clear Optical Fiber}

Kuraray also manufactures the clear optical fiber. These fibers are $1.2 \mathrm{~mm}$ diameter, S-35 Kuraray multi-clad optical fiber. Clear optical fibers in the ID are typically about $1.4 \mathrm{~m}$ for ID strips. For OD strips, the length is generally $1.2 \mathrm{~m}$. Clear fibers are then bundled together into eight-fiber connectors that mate to a connector on a PMT box.

\subsubsection{Material Composition of the Tracker}

To make cross-section measurements using the tracker as a target, we must have a full accounting of all material present in each tracker plane. The scintillator is composed primarily of carbon (C) and hydrogen (H) in nearly equal amounts, but other elements are present in the amounts listed in Table 3.1. These elements are contained in the optical fiber, epoxy, and light sealing materials used in construction of each plane. A careful study was done to assess the relative abundance of each element. A total estimate for each is found in Table 3.1. 


\begin{tabular}{c|c}
\hline \hline Element & Atoms $/ \mathrm{cm}^{2} /$ plane \\
\hline $\mathrm{C}$ & $0.889 \times 10^{23}$ \\
$\mathrm{H}$ & $0.903 \times 10^{23}$ \\
$\mathrm{O}$ & $0.024 \times 10^{23}$ \\
$\mathrm{Ti}$ & $1.76 \times 10^{20}$ \\
$\mathrm{Al}$ & $1.11 \times 10^{20}$ \\
$\mathrm{Si}$ & $1.29 \times 10^{20}$ \\
$\mathrm{Cl}$ & $1.89 \times 10^{20}$ \\
\hline \hline
\end{tabular}

Table 3.1: Number of atoms by type per $\mathrm{cm}^{2}$ per plane in the fiducial volume.

\subsection{MINER $\nu \mathrm{A}$ PMTs}

MINER $\nu$ A uses 64-anode Hamamatsu R5900-00-m64 PMTs. Each PMT is housed in a PMT box, like the one depicted in Figure 3.5. A PMT box contains eight connectors, each of which can mate to an eight-fiber connector allowing for a total of 64 fiber connections. Inside the box is a weave of 64 fibers that mate to a cookie, which is a plastic component that aligns the fibers. The weave is arranged in such a way to mitigate optical cross-talk between neighboring channels. The cookie mates fibers to individual PMT pixels. These pixels are arranged in a $8 \times 8$ grid with $2 \mathrm{~mm}$ pitch.

Light from a fiber illuminates a pixel, which can in turn liberate electrons from the anode via the photoelectric effect. The PMT has 12 stages; in each stage electrons are accelerated across a voltage gradient and directed upon an additional anode. This has the effect of multiplying the initial signal by a factor on the order of $10^{5}$ to $10^{6}$ per single photoelectron [77]. The ratio of end number of electrons over the initial number of photoelectrons is refered to as the gain. 


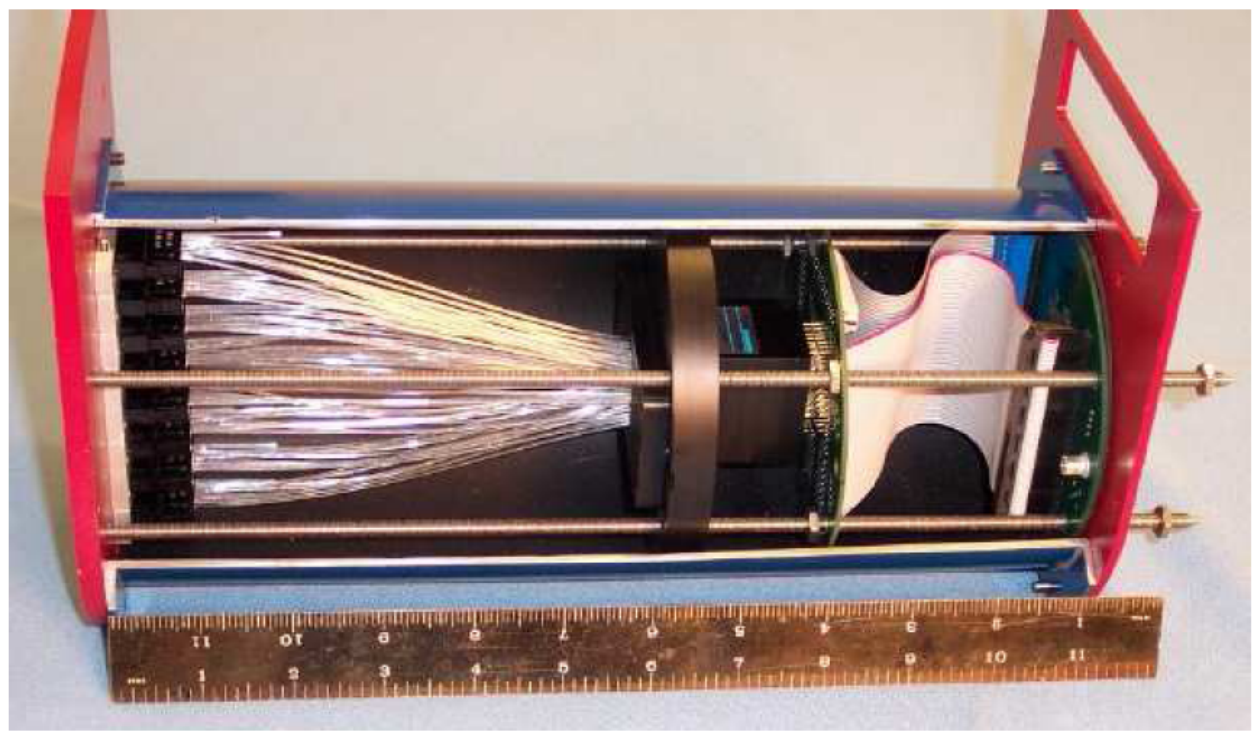

Figure 3.5: A PMT box containing a weave of fibers connecting to a 64-anode Hamamatsu PMT.

\subsection{MINER $\nu$ A Electronics and Data Acquisition}

The MINER $\nu$ A electronics and Data Acquisition (DAQ) systems control operations of the detector, readout neutrino interaction and calibration data, monitor data quality, and perform the initial event building. These systems ensure high live-time and quality data-taking.

Some differences exist between the DAQ systems used to take Frozen Detector data and the systems used to take data after the full build-out of the detector. The electronics used during the two run periods are the same. The DAQ software and operating systems differ between these periods. A comprehensive description of the LINUX-based DAQ system in use since the completion of the Frozen Detector run and a discussion of the MINER $\nu \mathrm{A}$ Front End Boards (FEBs) and rack mounted electronics can be found elsewhere [78]. 


\begin{tabular}{c|c}
\hline \hline Gain & Charge/ADC \\
\hline Low Gain & $1.25 \mathrm{fC} / \mathrm{ADC}$ \\
Medium Gain & $4 \mathrm{fC} / \mathrm{ADC}$ \\
High Gain & $15.6 \mathrm{fC} / \mathrm{ADC}$ \\
\hline \hline
\end{tabular}

Table 3.2: Number fC/ADC count for different FEB gains.

\subsubsection{MINER $\nu$ A Front End Boards}

Each PMT has a FEB mounted on the end of the PMT box opposite the clear fiber connectors. The FEBs serve several purposes. The FEBs each contain a CockroftWalton $(\mathrm{CW})$ necessary to generate the high voltages that power the PMTs. A FEB also contains six Application-Specific Integrated Circuit (ASIC) chips referred to as TriP-t chips. The TriP-t chips integrate signal charge from the PMT using 12-bit Analog-to-Digital Converter (ADC) units. The ADC is followed by a pipeline to store integrated charge. Each of the six TriP-t chips has 32 channels which service low, medium, and high gain channels. The high gain channels determine if a discriminator on the TriP-t passes the threshold. The threshold is a minimum amount of intergrated charge that must be surpassed for a channel to have a time associated with it. The initial signal is divided between each gain at input. This is to increase the total dynamic range of charge that can be measured by an FEB, which in turn increases the maximum amount of energy that can be measured on a channel while maintaining sensitivity to single photoelectrons. The number of $\mathrm{fC} / \mathrm{ADC}$ count for the different gains is shown in Table 3.2.

Each FEB also houses a Spartan 3E Field-Programmable Gate Array (FPGA). This unit controls FEB behavior, timing, and communications with the exterior electronics. These communications are accomplished by bit-by-bit frame relay. Individual frames contain header information with FEB addresses, timing information, 
various commands, and data. The addresses are necessary to distinguish FEBs once they are daisy-chained together. Communications occur over these chains using Low Voltage Differential Signaling (LVDS) through UTP ethernet networking cables. The FEBs are directly connected to a VME module described in Section 3.7.2.

The smallest unit of time discernible within MINER $\nu \mathrm{A}$ is set by FEBs and a timing module described in Section 3.7.2. The FEBs use a unit of time referred to as a clock-tick. One clock-tick is 9.4 ns, controlled by an on-board crystal oscillator at $53 \mathrm{MHz}$, chosen to match electronics clock of the Main Injector. By examining the clock-phase, FPGA registers have an effective granularity of a quarter of a clocktick giving an overall granularity of $2.4 \mathrm{~ns}$ for timing from the discriminators. The overall time is found by first initializing the internal clock counter on the FEB to a particular value. These values are set such that all FEBs have the same real start time. This is necessary since signals have different propagation times depending upon where a channel is in the detector. Different regions of the detector involve different pathlengths for a signal. The time of an interaction is then found by counting clock-ticks and taking into account the added granularity gained from the FPGA.

\section{Multiple Interactions and Deadtime}

Multiple interactions can be recorded on the same channel during any particular beam spill. Beam spills are roughly ten microseconds long, but the integration gate extends 16 microseconds to capture post-spill muon decays. If a signal crosses the discriminator threshold on a particular channel, timing and ADC information must be pushed into the registers before additional interactions can be recorded. A particular channel, is serviced by two TriP-t chips, with high and medium gain 
channels serviced by one TriP-t chip and the low gain channel handled by a separate TriP-t chip. During this push all 32 channels on both TriP-t chips servicing a channel are unable to integrate charge. This process takes 20 clock ticks $(\approx 188 \mathrm{~ns})$ to complete. We refer to this period where charge cannot be integrated as deadtime. If another interaction occurs on these channels during this deadtime, a fraction of or potentially none of the charge for a particular channel will be integrated.

Another form of deadtime can occur if too many interactions occur within a beam spill. The FEBs have a maximum number of times they can push data to the registers. This maximum number of pushes allows for five timed hits and one untimed hit for Frozen Detector running and eight (seven plus one) for recent running. Any charge integrated after the maximum number of timed pushes occurs will not have timing information and is not used for this analysis.

\subsubsection{Rack Mounted Electronics}

Rack-mounted electronics serve as a bridge between the computer that runs the DAQ and the FEBs that readout the PMTs. A chain of FEBs connects to a Chain ReadOut Controller (CROC) module at both ends of the daisy chain [79]. The CROCs are installed within a VME crate [80]. Each CROC can connect to four chains of FEBs. Chains of FEBs typically contain 10 FEBs, but this number can vary slightly by detector region. CROCs contain $6 \mathrm{kB}$ of dual-port memory for each chain. This memory stores frames that are exchanged between the DAQ and individual FEBs. Another module within the VME crate, the CROC Interface Module (CRIM), sends timing and trigger information to the CROCs. Each CRIM can be connected to four CROCs [81]. The VME crate contains a CAEN V2718 crate controller that controls the various modules within the crate [82]. In total, 
two VME crates, 8 CROCs, and 272 FEBs were active during the Frozen Detector analysis run.

A module within the VME crate, known as the MINER $\nu \mathrm{A}$ Timing Module (MvTM), sends trigger and gate information to the CRIM modules. This module is not controlled by the VME crate and contains a $53.1 \mathrm{MHz}$ crystal which is used as an internal clock for the module. A clock tick within the MvTM is $\approx 19$ ns. This frequency is selected to match the length of a FEB clock tick, where a FEB clock tick is twice the period for this frequency, or 9.4 ns as described in Section 3.7.1. The MvTM, which uses a modified MINOS timing card [83], distributes the Main Injector timing signal.

During the Frozen Detector era of running, this module received information from the Main Injector regarding the start of a NuMI spill. It then sent a trigger to the CRIM, which sent a command to its CROCs to open the FEB integration gates so neutrino interactions could be recorded. See Section 2.2 for details on NuMI beam spills. The gate over which MINER $\nu \mathrm{A}$ records events opens 0.5 microseconds before the NuMI spill and remains open 5.5 microseconds after the spill. The gate is kept open for an extended amount of time to remain sensitive to Michel electrons from stopping muons and pions. A muon has a mean lifetime of 2.2 microseonds, meaning that activity can be present in the detector for many microseconds after the last neutrino interaction. A computer running the DAQ program reads out data from the CROC module.

\subsubsection{The Data Acquistion Computer}

The computer running the DAQ program is different for Frozen Detector running and running after completion of construction of the detector. During running for 
the Frozen Detector data set, data are taken with the DAQ program running on a computer using Windows Vista [84]. Since this period data are recorded using a computer running Fermi Linux [85].

Data runs and other special runs are initiated from a custom written DAQ program on the DAQ computer. An experimenter monitoring operation of the detector oversees the start of a run, data taking, the end of the run, and monitors the quality of the data.

\subsubsection{Data Taking Procedures}

At the completion of the gate, frames containing the voltage levels, timing, FPGA register information, and the charge recorded on low, medium, and high gain ADCs on a FEB are readout. For the Frozen Detector data set, all FEBs are readout. For the period after completion of construction of the detector, only FEBs where a discriminator threshold is crossed are readout.

A discriminator threshold is crossed when the integrated charge on a high-gain channel passes a set threshold. For Frozen Detector running, up to five different sets of time-stamped ADC activity on a given FEB are readout for a given gate. These frames are transferred from the FEBs to memory located in the CROC before being readout by the computer running the DAQ program.

Data from MINOS are taken and processed by the MINOS collaboration and later provided to MINER $\nu$ A for offline processing. A description of MINOS electronics is given elsewhere [86]. 


\subsubsection{Special Calibration Gates}

During the Frozen Detector running, dedicated calibration runs were taken. These special runs included Light Injection (LI) runs as well as Pedestal runs. Due to the configuration of the DAQ at the time of this particular data run, these types of runs had to be taken in dedicated periods of running and more specifically, not concurrently with data running. These runs were often taken opportunistically, such as when the NuMI beam was not receiving protons from the Main Injector. At other times dedicated runs were taken even though beam was available.

\section{Pedestal Gates}

Each channel has a low level of observable signal present, and this offset is commonly referred to as the pedestal. To properly measure the energy deposited in a channel, this pedestal must be subtracted. Because the level of the pedestal on different channels can drift over time, special runs are taken periodically to ensure that accurate information on the pedestal is available for use in later subtraction.

\section{Light Injection Gates}

Special LI gates are used to ensure that each PMT voltage is properly calibrated. Since PMT gains may drift, periodic LI runs must be taken. A LI box sends signals from Light Emitting Diodes (LED) over a clear optical fiber to a special connection on the PMT box. Fits to LI data allow the gains to be extracted for a particular interval of time. 


\subsubsection{Nearline Data Monitoring}

Data monitoring took place on a Near-Online (Nearline) system. This system involves low level, realtime data processing on a server running Fermi Linux. Low-level hit and timing information are inspected to ensure that all channels are active and operating in the nominal way. This information includes maps of activity across electronics channels. Collaborators performing control shifts monitor these plots and look for problems such as a malfunctioning FEB or a PMT exhibiting hardware problems. Close monitoring is vital, particularly so during the Frozen Detector running. Seepage of water out of the rock in the ceiling of the cavern that is the Near Detector Hall would "rain" on the FEBs, which are exposed at the top of the detector. This caused occasional FEB malfunction, which a shifter could spot with the use of nearline monitoring plots.

Other monitoring plots include the average of PMT voltages, the distribution of all hits in time, and the average number of ADC counts on low, medium, and high gain channels. A PMT with a voltage too far from the average voltage can be an issue, as this can cause non-optimum gains or potentially damage to the PMT itself. The distribution of hits can be used to ensure that the interactions over time reflect the spill structure of the beam. This is essential to ensure that a MINER $\nu$ A gate is properly aligned to a NuMI spill. Last, reasonable distributributions of ADC counts on low, medium, and high gain channels verify that activity in the detector is being properly measured. 


\subsection{Descriptions of the MINOS Detector}

The MINOS Near Detector is a magnetized sampling detector located approximately two meters downstream from the MINER $\nu$ A detector. The MINOS Near Detector is part of a larger neutrino oscillation experiment with a far detector located in a mine in Northern Minnesota. The MINOS Near Detector, hereafter referred to simply as MINOS, is composed of alternating planes of steel and scintillator.

\subsubsection{MINOS Planes}

The first MINOS plane after the air gap between MINOS and MINER $\nu \mathrm{A}$ is a 2.54 cm steel plane. The MINOS detector has a tracking region and a calorimeter region following this. In all regions, MINOS scintillator planes are $1 \mathrm{~cm}$ thick while steel planes are $2.54 \mathrm{~cm}$ thick. MINOS scintillator planes have the same composition as MINER $\nu$ A scintillator. MINOS planes have an irregular shape as shown in Figure 3.6. Each plane has a hole for the coil that creates the magnetic field within MINOS.

In the upstream tracking portion of the detector, each steel plane has a scintillator plane affixed to its upstream edge with a total of 120 such planes. These planes alternate in view, but with only two unique views, U and V. MINOS U and V planes do not have the same orientation as MINER $\nu \mathrm{A} \mathrm{U}$ and $\mathrm{V}$ planes. MINOS $\mathrm{U}$ and $\mathrm{V}$ planes are rotated $45^{\circ}$ clockwise and counterclockwise from the vertical axis. Planes in this upstream region are either "partially instrumented" or "fully instrumented." These terms refer to the fraction of the plane the scintillator covers, with partially instrumented planes having a smaller fraction of coverage of the steel plane.

Following this region only one of every five of the remaining 141 steel planes has 
a an instrumented scintillator plane attached to the upstream edge to the steel [87]. In this region only fully instrumented plane are used. Fewer instrumented planes are included in this region since trajectory information of a track is not as important as in the upstream regions of the detector.

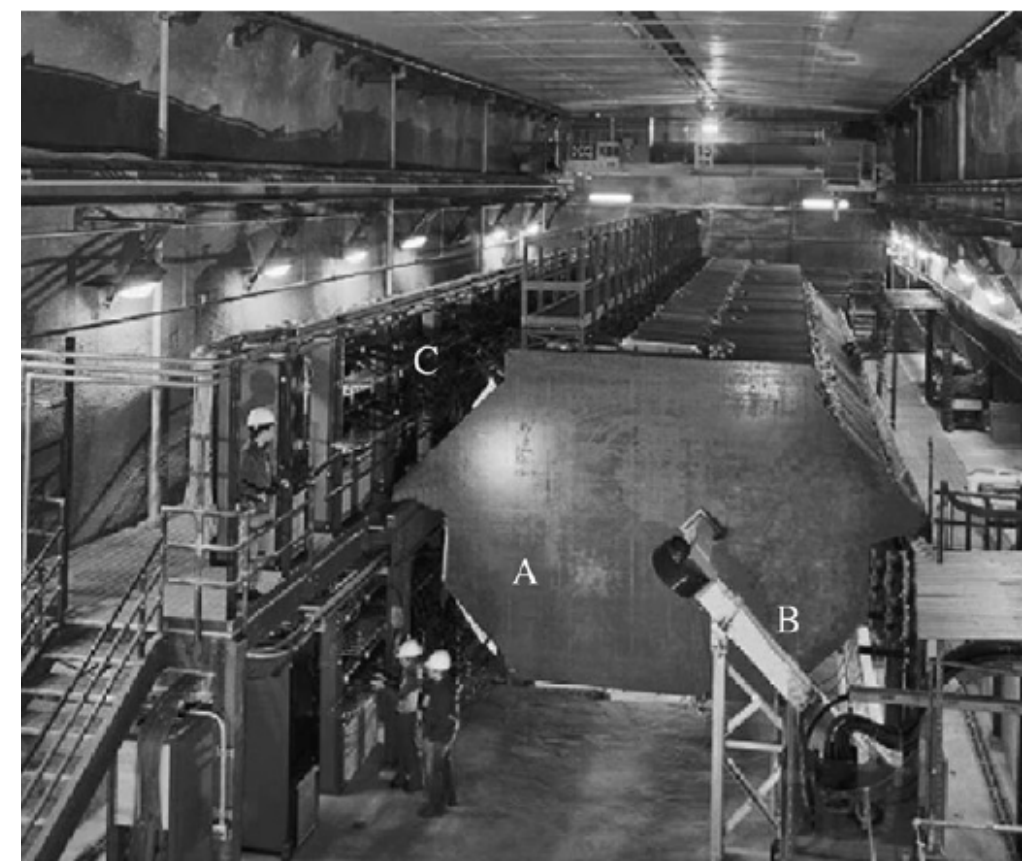

Figure 3.6: The MINOS Near Detector prior to installation of the MINER $\nu$ A from [87].

\subsubsection{The MINOS Coil}

A coil running through the center of MINOS and back along the edge of the detector generates a toroidal magnetic field with an average strength of $1.28 \mathrm{~T}$ in the steel planes. A current of $40 \mathrm{kA}$ is used to generate this field. Due to resistive heating from this large current, the coil is water cooled to maintain a safe operating temperature.

The purpose of the coil is to make momentum and charge measurements of particles that traverse the detector. The polarity of the current in the field can be 
switched, so as to better contain either positive or negative muons. This polarity is changed depending on whether FHC or RHC running is taking place. Examining which direction a particle curves in the magnetic field allows an accurate method of assessing the charge. In measuring the curvature of the track, one can also find the momentum of the particle [87].

\subsection{The ArgoNeuT Detector}

ArgoNeuT, shown in Figure 3.7, is a prototype liquid argon time projection chamber detector that resided between the MINOS and MINER $\nu$ A detector for roughly $95 \%$ of the Frozen Detector run. The detector has a cryogenic insulated vessel filled with liquid argon. The detector contains components associated with the cyrogenics along with supporting steel structures that support the aparatus [88]. The presence of this detector is a source of energy loss for muons as they travel from MINER $\nu \mathrm{A}$ to MINOS along with a source of smearing in the energy resolution during this era of running. 


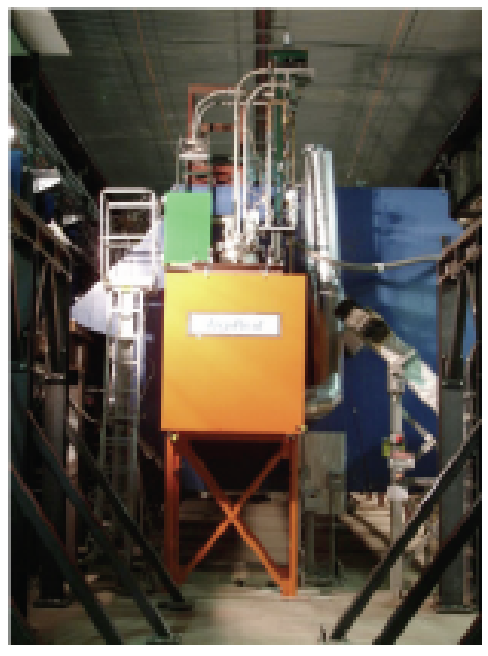

Figure 3.7: A picture of the fully installed ArgoNeuT detector that resided between MINER $\nu \mathrm{A}$ and the MINOS Near Detector [88]. 


\section{Chapter 4}

\section{Reconstructing and Simulating}

\section{Neutrino Interactions in}

\section{MINER $\nu \mathrm{A}$}

MINER $\nu \mathrm{A}$ reconstruction can be characterized as either low level or high level reconstruction. Low level reconstruction is performed only for data. High level reconstruction is identical for data and the simulation. In addition to the reconstruction, we simulate each component of the MINER $\nu$ A detector and each step of processing. We also have unique simulations of the beamline, MINER $\nu \mathrm{A}$, the MINOS detector, and ArgoNeuT.

\subsection{Low Level Data Processing}

The data that we read out from the detector is initially in long unbroken frames of binary that need to be unpacked into a more readily usable structures. After 
this unpacking, we assemble data objects corresponding to a particular channel. Any channel with integrated charge above the discriminator threshold for the high gain we refer to as a hit. The reconstruction schemes we use process these hits into more complex objects. The time for a hit is found by looking at the time the discriminator threshold is passed. The next level of processing includes performing pedestal subtraction and applying all relevant calibrations to each channel.

We also unpack information for the entire gate, which corresponds to a single NuMI spill. After unpacking, each gate contains all relevant MINER $\nu \mathrm{A}$ detector information for that spill. This includes a record of any hardware problems, information for each channel of the detector, and relevant timing information.

\subsubsection{Pedestal Subtraction}

We describe the procedure for taking pedestal measurements in Section 3.7.5. Examples of high gain pedestals are shown in Figures 4.1 and 4.2, with the latter containing a background hit potentially from a cosmic ray. When subtracting pedestals, outliers that come from likely background activity are removed before finding the appropriate value for the pedestal to subtract. Since pedestals can potentially change over time, a search is performed to find pedestal data closest in time to the data. Pedestals are subtracted for low, medium, and high gain channels. This is performed prior to all other processing and calibrations.

\subsubsection{Applying Calibrations}

We apply a variety of calibrations to each channel which we access from a database. Here calibrations include the factor for converting ADC counts to energy. We also 


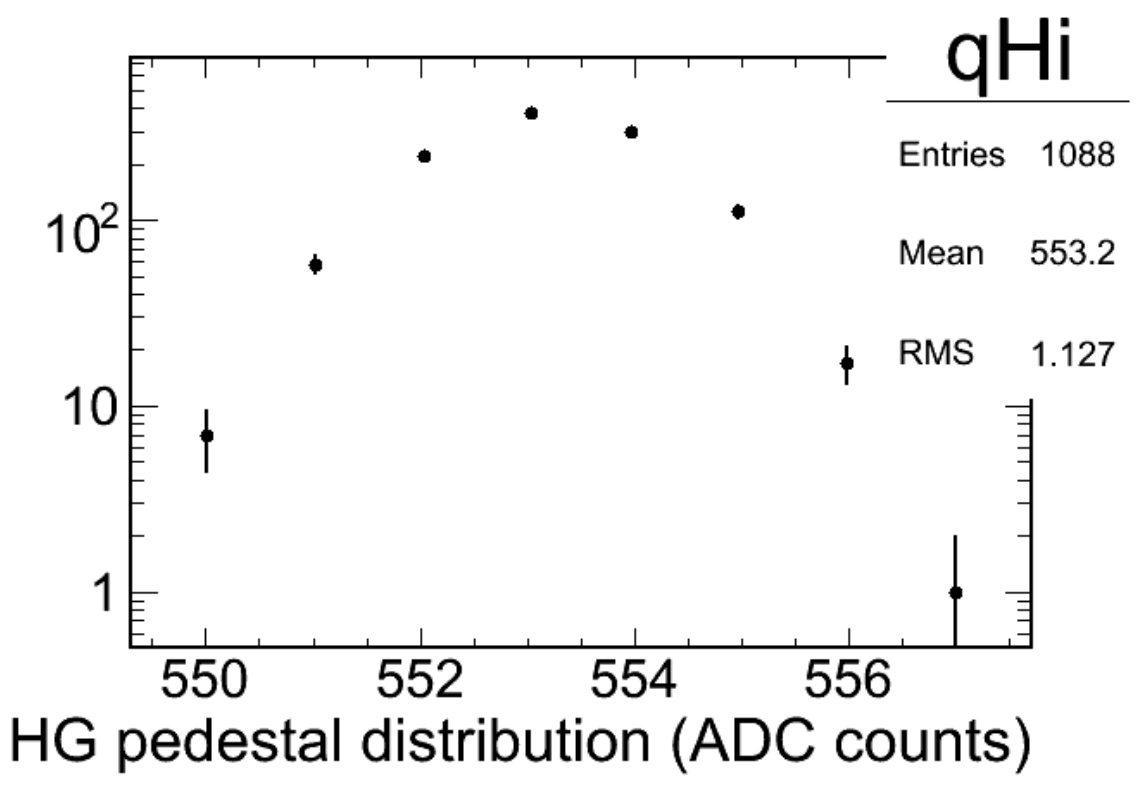

Figure 4.1: High gain pedestal. This plot was made by A. McGowan of the MINER $\nu \mathrm{A}$ collaboration.

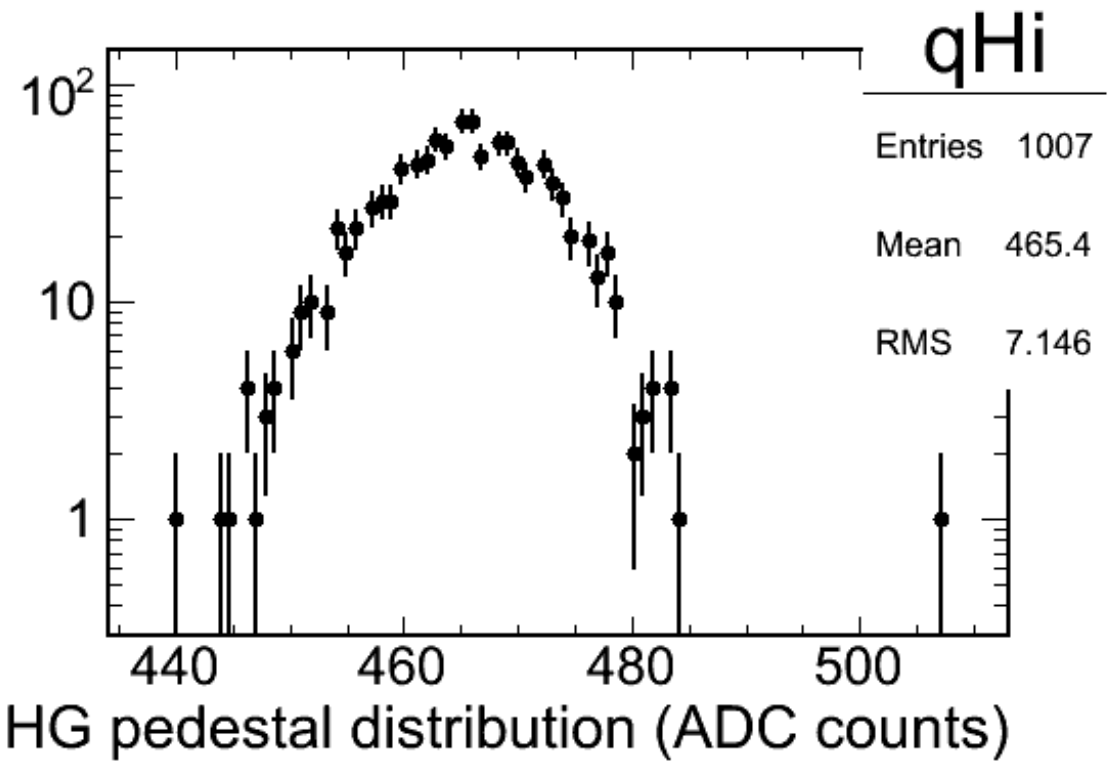

Figure 4.2: High gain pedestal with a background hit near 508 ADC count, which could potentially be from a cosmic ray. This plot was made by A. McGowan of the MINER $\nu \mathrm{A}$ collaboration. 
apply calibrations for FEBs, for different PMT gains, for variations between strips within the detector, for timing misalignment and smearing, and for variations in the observed strip response to muons (Muon Energy Unit, MEU).

\section{FEB Calibrations}

Prior to installation, all FEBs undergo charge injection tests. This involves injecting known amounts of charge into each FEB and monitoring the response in the high, medium, and low gains for each channel. A fit is done using a tri-linear function to parameterize the response for each of the gains on each channel. We use a trilinear fit since we observe up to three different linear regions with unique slopes when looking at the number of ADC counts as a function of charge. The linear regions are separated by sharp transitions or kinks. The fit returns both the slopes in units of $\mathrm{ADC} / \mathrm{fC}$ and the locations of the kinks that are the boundaries between the different slopes. An example of a tri-linear fit for all 64 channels on an FEB is shown in Figure 4.3.

When performing these fits, care is taken to stay away from the saturation point. We apply these fitted parameters as calibrations to map the number of ADC counts to a particular charge. Since the slopes and the transition points, or kinks, can differ from channel to channel, this correction must be applied or non-linear effects could be observed in later steps of processing.

\section{PMT Gain Calibrations}

Gains for the PMT can very over time and with temperature. To account for this variation, frequent calibrations are applied. We find calibrations for PMT gains by using light injection data which we take on the order of once per day. We find the 


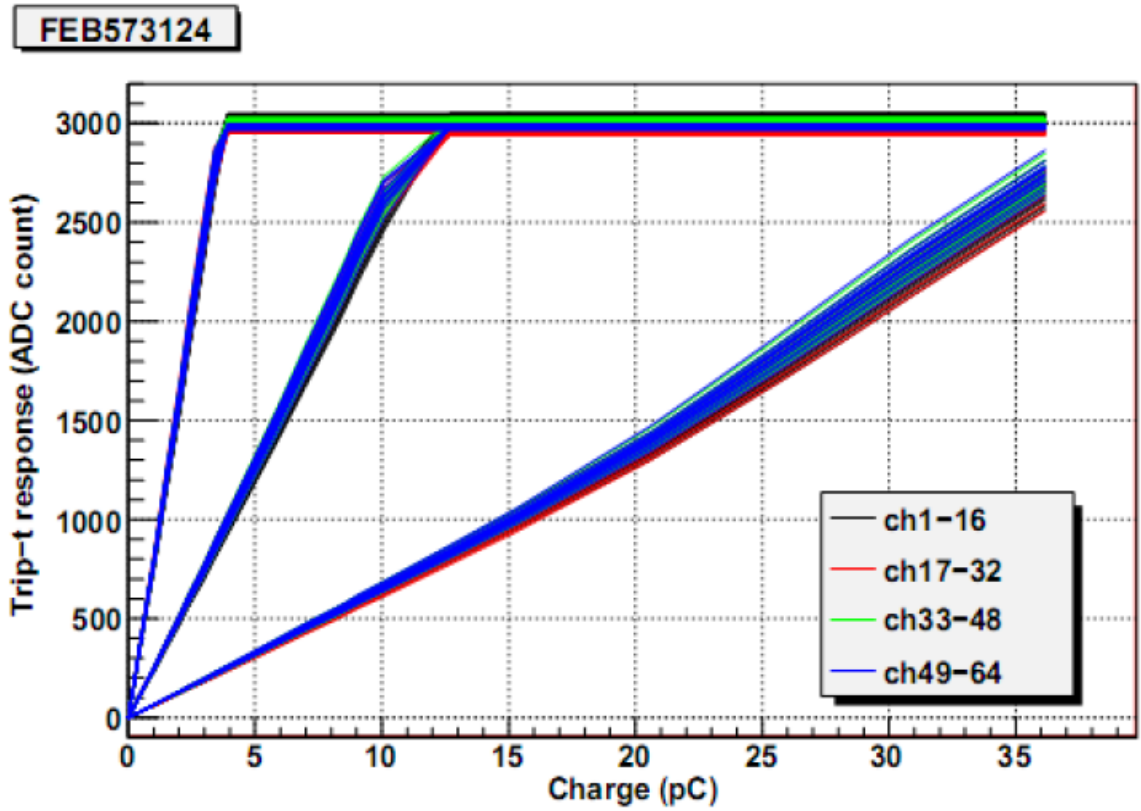

Figure 4.3: Tri-linear fits to characterize high, medium, and low gains for each channel on a particular FEB. This plot was made by I. Danko of the MINER $\nu$ A collaboration.

gain for a channel by performing a fit using these data. The fit function, shown in Equation 4.1, incorporates $\sigma_{\mathrm{Q}}^{2}$, the variance of the number of photoelectrons arriving at the first PMT dynode, $\sigma_{\mathrm{p}}^{2}$, the variance of the single photoelectron peak, $\overline{\mathrm{Q}}$, the variance of the pedestal, and $\mathrm{w}^{2}(\mathrm{~g})$ given in Equation 4.2 , which is a function of the gains at each of the PMT stages. Note that the gain at each dynode stage is a function of the voltage at that stage, as shown in Equation 4.3.

$$
\begin{gathered}
\mathrm{g}=\frac{\sigma_{\mathrm{Q}}^{2}-\sigma_{\mathrm{p}}^{2}}{\overline{\mathrm{Q}}\left(1+\mathrm{w}^{2}(\mathrm{~g})\right)} . \\
\mathrm{w}^{2}(\mathrm{~g})=\sum_{\mathrm{i}=1}^{12} \frac{1}{\prod_{\mathrm{k}=1}^{\mathrm{i}} \mathrm{g}_{\mathrm{k}}} .
\end{gathered}
$$


The value $g_{k}$ in Equation 4.2 is the gain at a particular dynode stage of the PMT, and can be found by

$$
g_{i}=g_{1}\left(\frac{V_{i}}{V_{1}}\right)^{\alpha},
$$

where $g_{1}$ is the gain for the first dynode in the PMT, $V_{i}$ is the voltage at stage $\mathrm{i}, \mathrm{V}_{1}$ is the voltage at the first dynode stage, and $\alpha$ is 0.75 based on data from Hamamatsu [77]. We use PMT gains to convert from the amount of calibrated charge to the number of photoelectrons. The distribution of PMT gains found with the above method is shown in Figure 4.4.

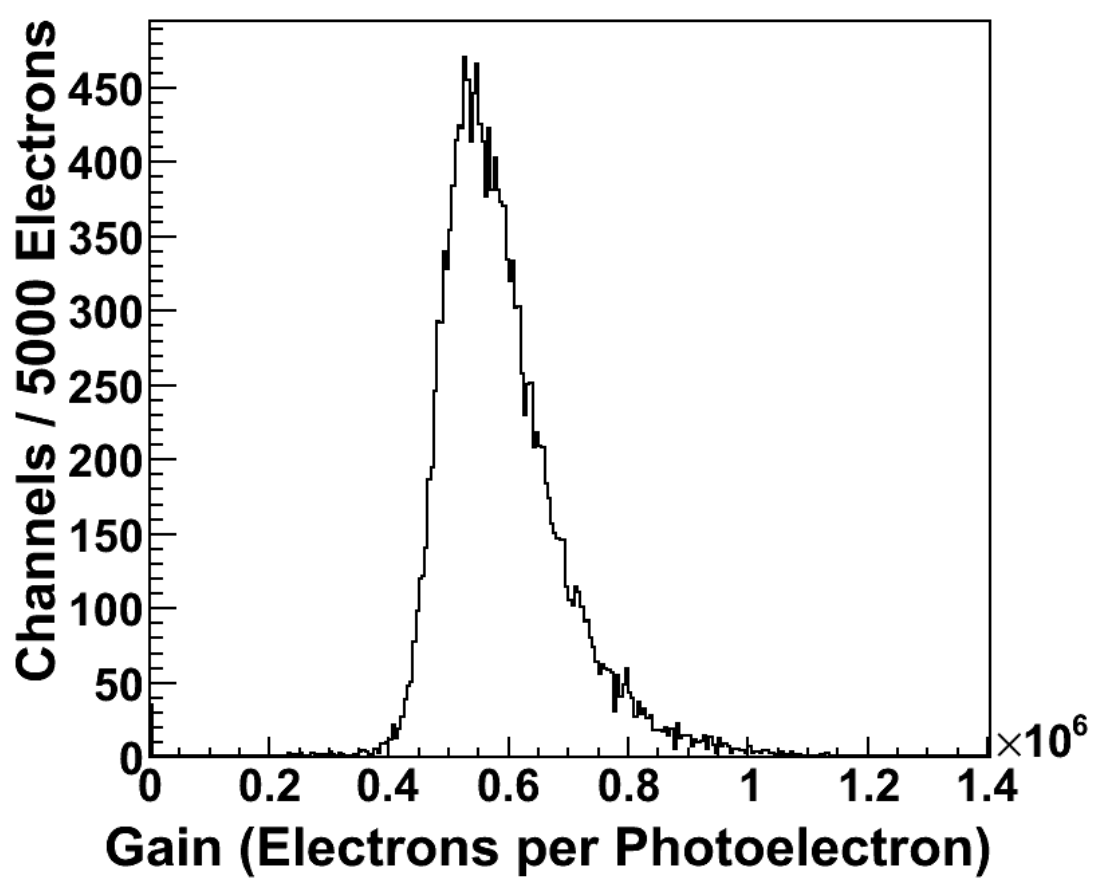

Figure 4.4: PMT gains for a particular time interval. This plot was made by B. Eberly of the MINER $\nu \mathrm{A}$ collaboration. 


\section{Calibrating for Variations between Strips}

We expect some variations to exist between strips. This could be due to variations in materials such as different batches of scintillator as well as other differences such as whether air bubbles exist in the epoxy used to fix the WLS fiber within the strip. A strip by strip correction is applied to account for this.

The calibration is found by using rock muons, which are muons generated by neutrino interactions in material upstream of the detector. Only rock muons matched to a muon in MINOS are used. We discuss the procedure for matching muons that pass out of the MINER $\nu \mathrm{A}$ detector to muons found in MINOS in Section 4.3.6. The amount of energy a muon should deposit in a strip per $\mathrm{cm}$ traversed is easily found. A multiplicative correction is applied to remove variations between strips in response to muons. Figure 4.5 shows the result of applying this calibration on observed value of $\frac{\mathrm{dE}}{\mathrm{dx}}$ in a strip.

\section{Timing Calibration}

Two different corrections for timing are made. Even after synchronizing FEBs, small timing offsets still exist between FEBs. The offsets are found and applied for each FEB.

In addition to FEB timing offsets, a correction for charge slewing is applied. Charge slewing occurs due variations in the time required to pass the discriminator threshold. Larger signals have a faster rising edge than smaller signals which results in large signals passing the discriminator threshold earlier than small signals would. This leads to systematic differences in time between small and large signals. An illustration of slewing is shown as a function of photoelectrons in Figure 4.6. The behavior of this timing offset as a function of charge is mapped out and used to apply 


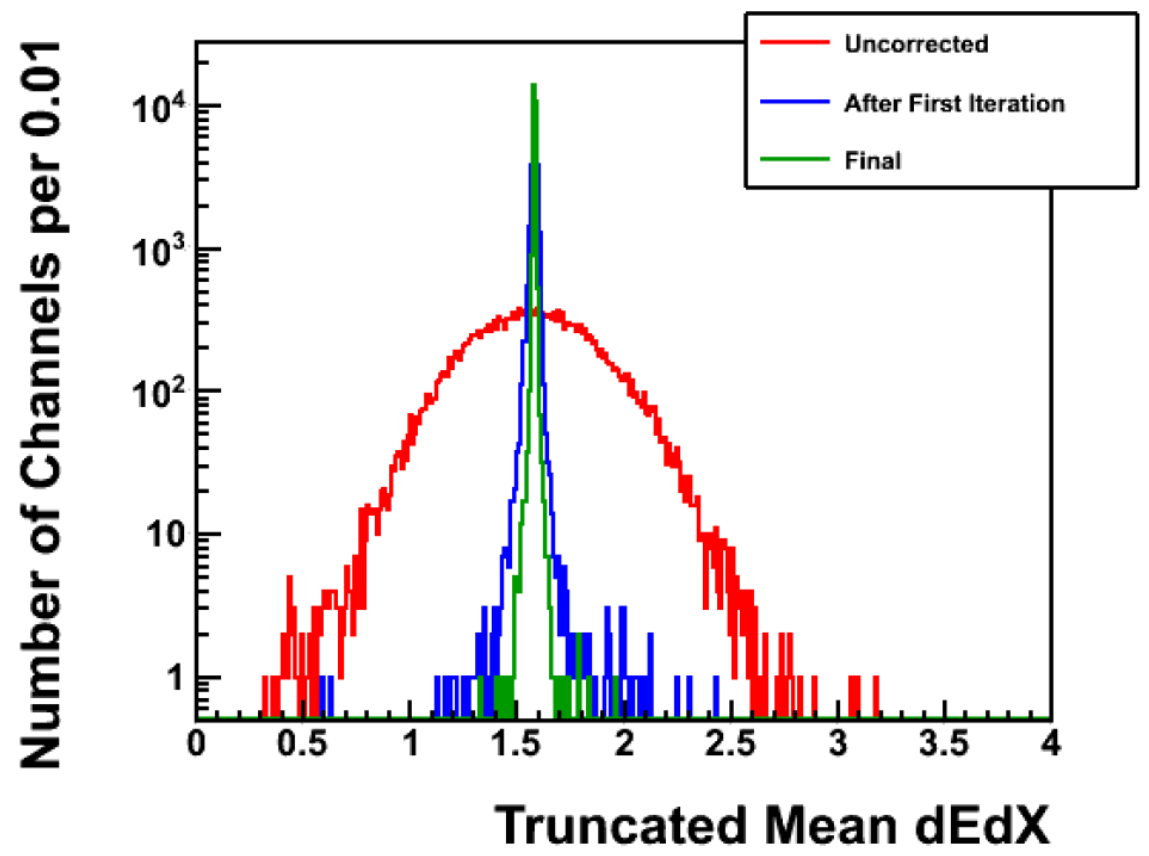

Figure 4.5: Result of applying the calibration for variatons between strips after the first and last iterations of this calibration compared to the uncalibrated distribution. This plot was made by C. Marshall of the MINER $\nu$ A collaboration.

a correction. The effect of applying the slewing correction in the Frozen Detector data set is shown in Figure 4.7. Charge slewing also changes over time since the level of slewing is dependent on the gains for a particular channel, which also can vary with time. As a consequence, slewing corrections are found for particular intervals of time.

\section{MEU Correction}

We also make a MEU calibration. Although similar in some respect to the correction made for variations between strips, this accounts for other time dependent effects such as the level of scintillator response to a particle decaying over time. The Near Detector Hall temperature is consistently higher than design leading to some 


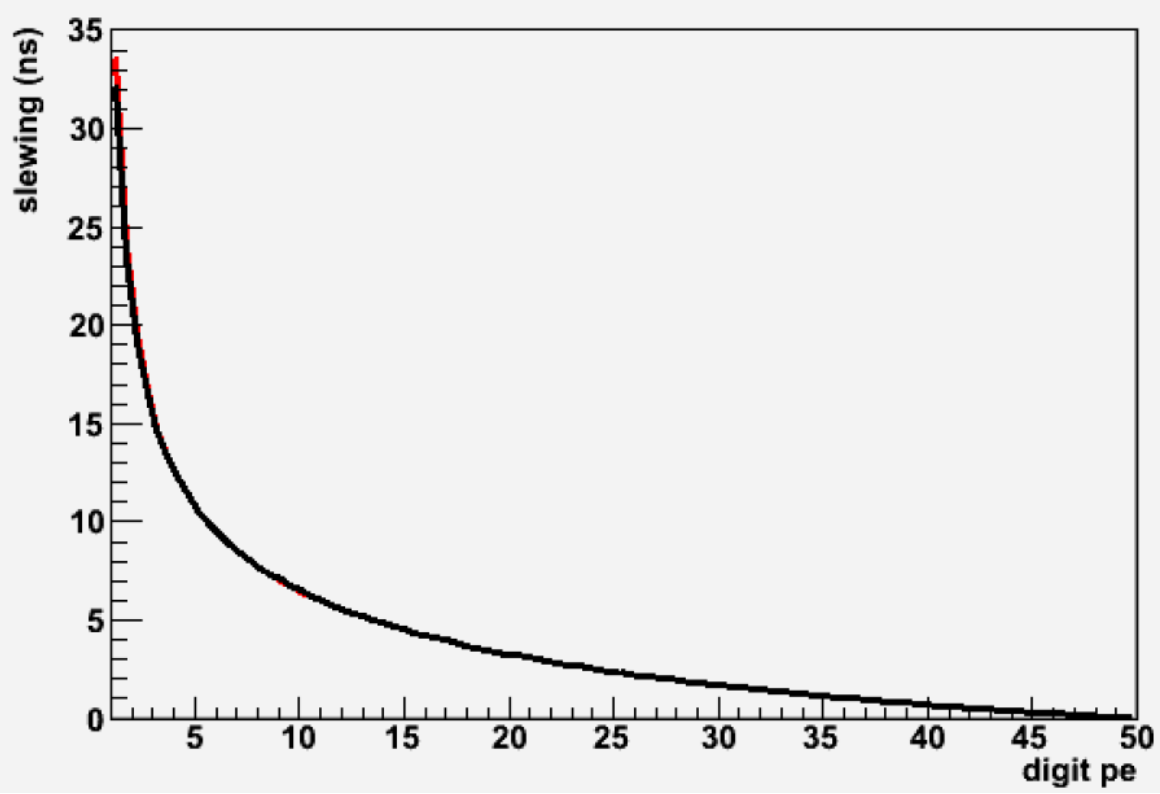

Figure 4.6: Charge slewing as a function of the number of photoelectrons. This plot was made by A. Mislivic of the MINER $\nu \mathrm{A}$ collaboration.

degredation of the scintillator response. As a consequence of this effect, the expected amount of light generated by a minimum ionizing particle will decline over time. Apart from the effects of scintillator degradation, we also expect the overall response to vary with temperature of the Near Detector Hall as well. The time dependence of muon response can be found in Figure 4.8 .

To make a correction for the variations in response, we use rock muons. We require these muon be matched to MINOS. A fit is done for different intervals of time of the peak energy for a rock muon in data compared to simulation using a fifth order polynomial. The result of the fit is used to correct for the MEU response in data. Data and simulation overlays of muon cluster energy after the MEU calibration are shown in Figure 4.9. 


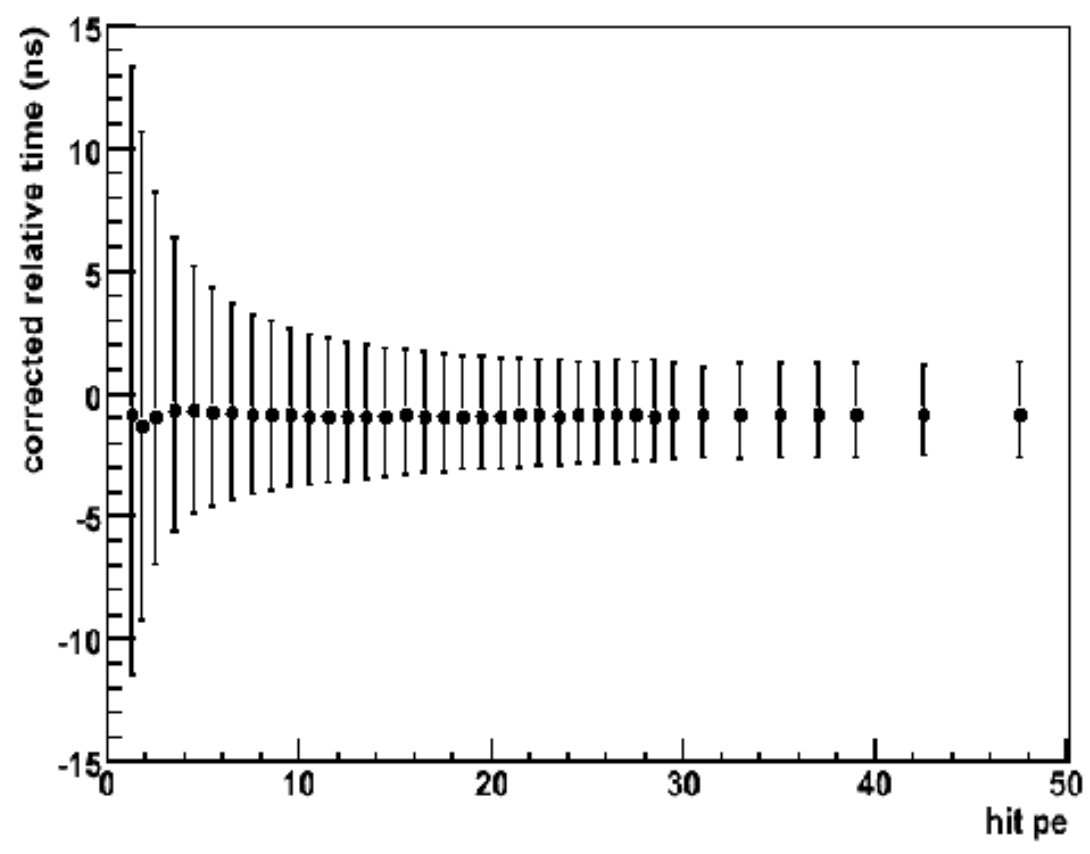

Figure 4.7: The time and resolution of a hit as a function of the number of photoelectrons after the slewing correction has been applied. Although the slewing effect has been removed we do observe a lower timing resolution for smaller signals. This plot was made by A. Mislivic of the MINER $\nu \mathrm{A}$ collaboration.

\section{Converting from ADC Counts to Energy}

ADC counts from a FEB channel need to be converted into an energy. ADC counts for low, medium, and high gains are inspected and a particular gain is selected for conversion to energy. The gain that is used depends upon the particular value of the charge found on a channel as given by the FEB calibrations. Different gains saturate at different points and have general ranges where they are approximately linear. Once a gain is selected, the above calibrations are applied to map ADC counts to charge, then photoelectrons, and then to an energy. 


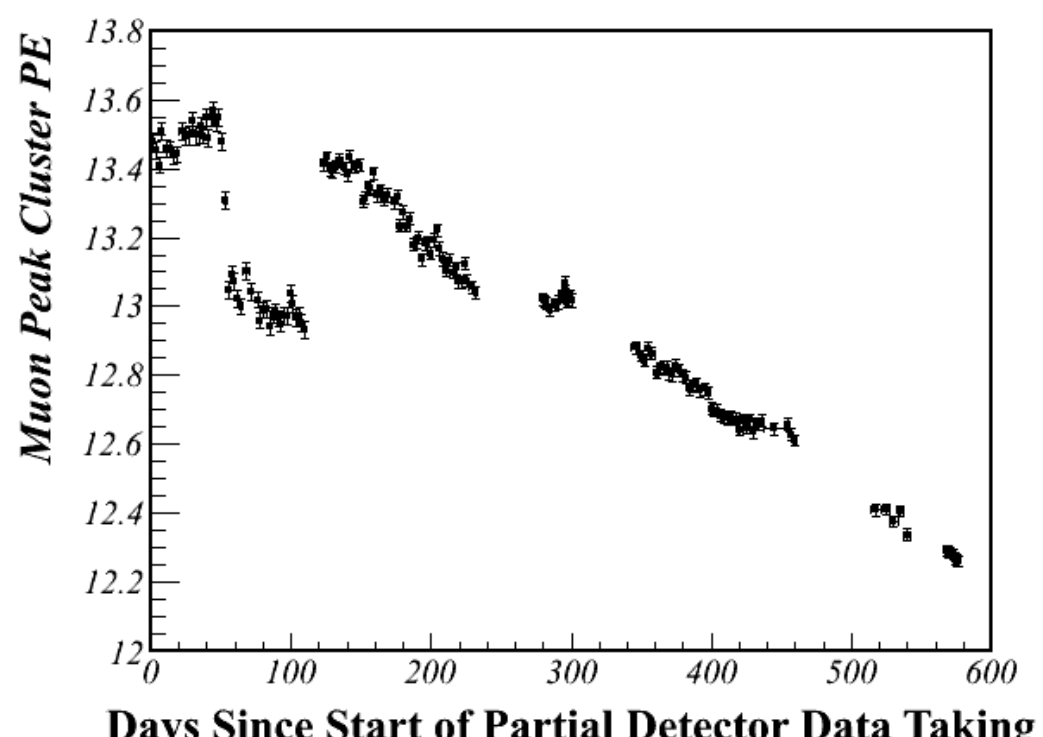

Figure 4.8: A plot of the peak number of photoelectrons for muons versus time. Note that two effects are present, temperature variations and degradation of the scintillator. Data from the Frozen Detector runs as well as later data taking periods are shown. The varying response to muons requires a time dependent MEU calibration to account for this. This plot was made by A. Mislivic of the MINER $\nu$ A collaboration.

\section{Search for Detector Misalignments}

We also look at other detector systems to assess whether calibrations are necessary. Specifically, we studied how well aligned the MINOS and MINER $\nu \mathrm{A}$ detectors are. We searched for unaccounted for offsets, rotations in the XY, XZ, and YZ planes, and looked for differences in the scales of the $\mathrm{X}, \mathrm{Y}$ and $\mathrm{Z}$ components of the coordinate systems of the two detectors. The method for studying these effects involves elements discussed in Section 4.3.6 on matching muons in MINER $\nu \mathrm{A}$ and MINOS and Section 4.3.4 on tracking in MINER $\nu \mathrm{A}$.

We performed these studies using muons matched to MINOS. To minimize effects from the MINOS magnetic field and multiple scattering, we consider only muons with 


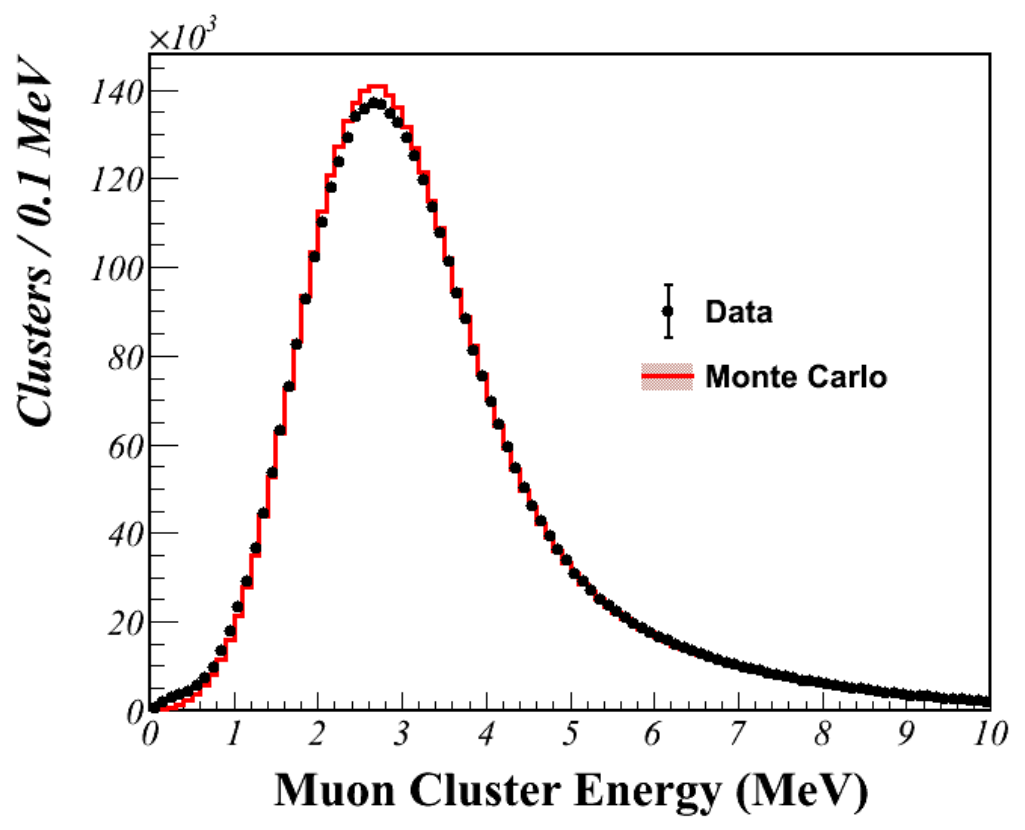

Figure 4.9: Data and simulation overlays of muon cluster energy after the MEU calibration is applied. This plot was made by A. Mislivic of the MINER $\nu$ A collaboration.

more than $10 \mathrm{GeV}$ of energy. We also require muons to be reconstructed through the last MINER $\nu \mathrm{A}$ plane and starting in the first MINOS plane. The method for find misalignments involved projecting a track from MINER $\nu \mathrm{A}$ to MINOS and looking at the residual between the projection and the position of the matching track, which we refer to as the match residual. If rotations, offsets, or differences in coordinate system scales exist, the structure should become apparent by looking for the matching residual to scale with different quantities. We also look at angle residuals between matched tracks to look for similar structure.

Corrections for offsets between the two coordinate systems in the $\mathrm{X}$ and $\mathrm{Y}$ directions are made. We use the mean of the match residuals for the $\mathrm{X}$ and $\mathrm{Y}$ coordinates as a straight forward way of ensuring the coordinate systems have a common origin in the XY plane. 
To find potential rotations in the XZ and YZ planes, we compare angles between matched muons in the two detectors. The difference of these two angles should be peaked at zero if no rotation is present. We find no observable rotation in either the XZ or YZ planes as shown in Figures 4.10 and 4.11. Since no observable rotation is present, no correction needs to be made.
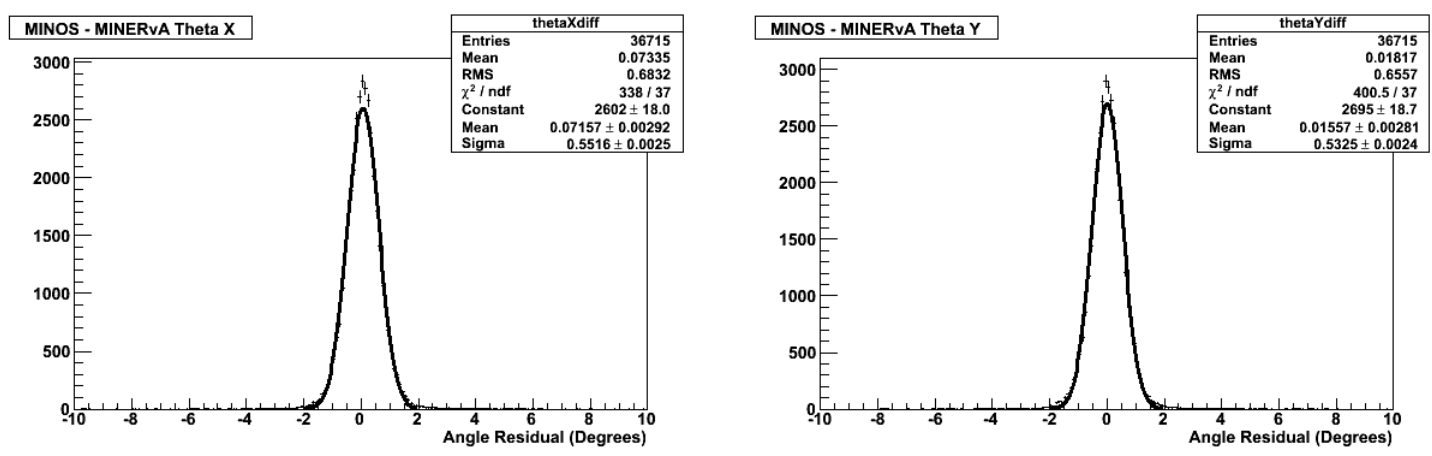

Figure 4.10: Plots of angle residuals between MINER $\nu \mathrm{A}$ and MINOS tracks in data. The residuals in data are peaked very closely to zero as given by the overall mean and the mean found with a fit.
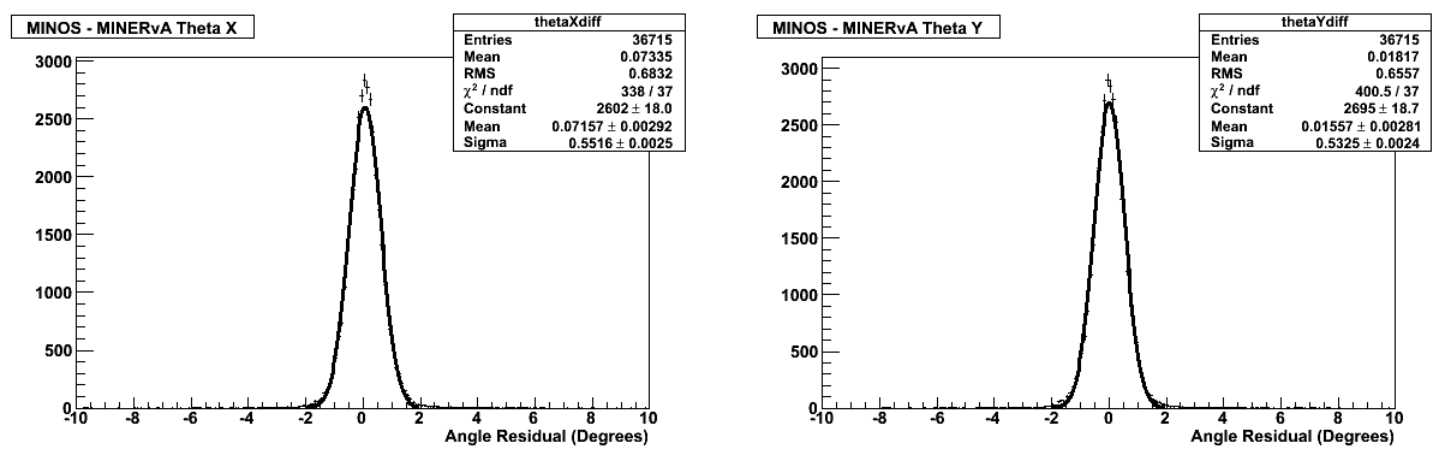

Figure 4.11: Plots of angle residuals between MINER $\nu \mathrm{A}$ and MINOS tracks in the simulation. The residuals in simulation are peaked very closely to zero as given by the overall mean and the mean found with a fit.

To look for scaling issues, we look at the angle residual as a function of the angle of the muon in MINER $\nu \mathrm{A}$. If a scale issue does exist, the angle residual should increase as the angle increases. Unfortunately, the distribution of muons that are 

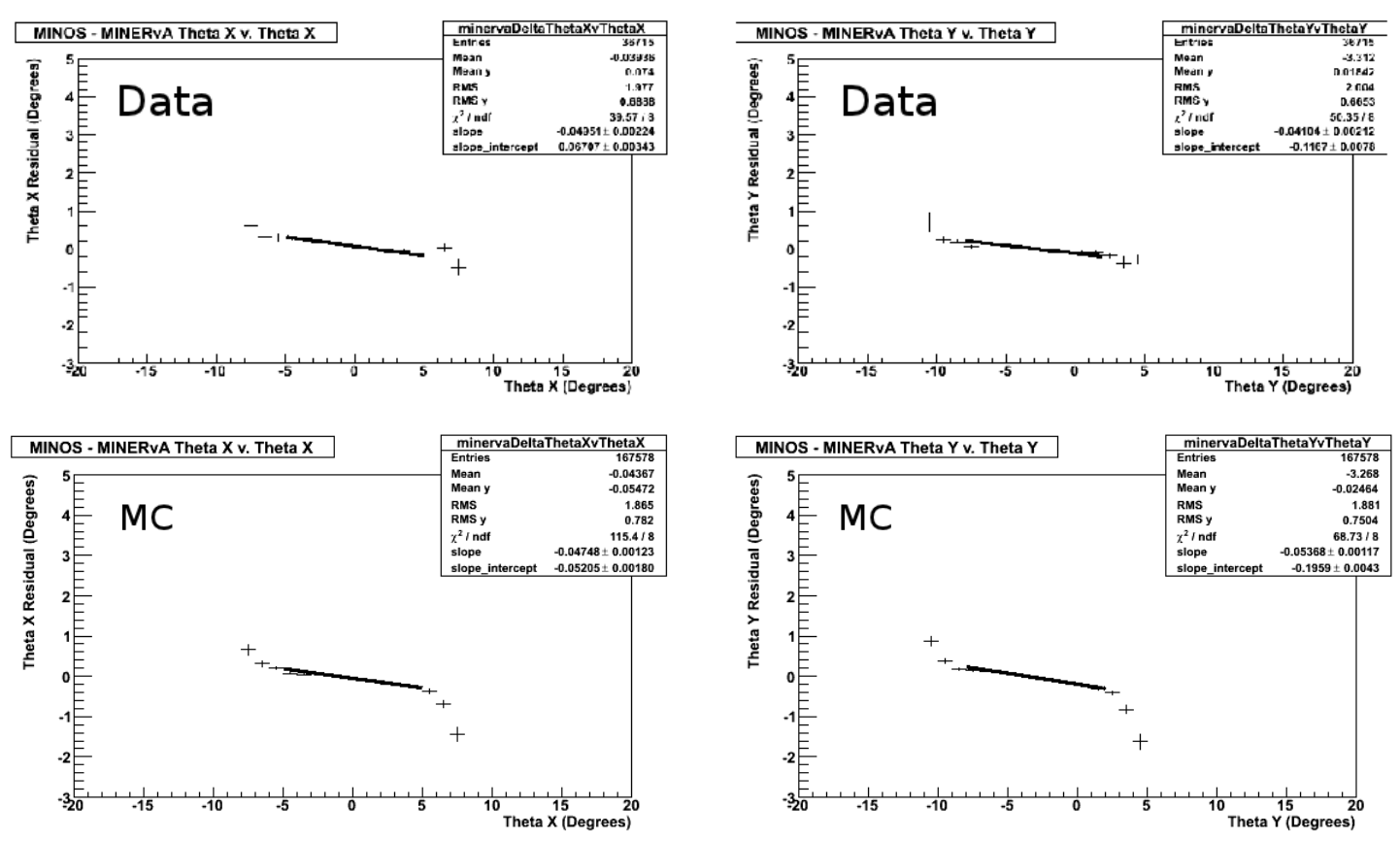

Figure 4.12: Plots searching for coordinate system scaling issues in data and Monte Carlo (MC). The slope is due to acceptance issues. We find no substantial differences in slope.

matched to MINOS are shaped by angular acceptance effects. This causes some slope to exist even in the simulation which should not have a scale issue by construction. Thus to search for a scale issue, we compare the angle residual versus angle plots in data and simulation. These comparisons are make in Figure 4.12. We do not see an appreciable difference in slope meaning no large scale issues exist and no correction needs to be made.

To determine if rotations in the XY plane exist between the MINOS and MINER $\nu \mathrm{A}$ detectors, we look at match residuals in the $\mathrm{X}(\mathrm{Y})$ coordinate as a function of the $\mathrm{Y}(\mathrm{X})$ coordinate. If the coordinate systems are not aligned, the residual should increase as function of the orthoganol coordiante. Consider the two dimensional rotation 


$$
\left(\begin{array}{c}
\mathrm{x}^{\prime} \\
\mathrm{y}^{\prime}
\end{array}\right)=\left(\begin{array}{cc}
\cos \theta & -\sin \theta \\
\sin \theta & \cos \theta
\end{array}\right)\left(\begin{array}{l}
\mathrm{x} \\
\mathrm{y}
\end{array}\right),
$$

where $\theta$ is the angle of rotation and $\mathrm{x}^{\prime}$ and $\mathrm{y}^{\prime}$ are the rotated coordinates. Multiplying through and using the small angle approximation where $\cos \theta \approx 1$ and $\sin \theta \approx \theta$, we find

$$
\mathrm{x}^{\prime}=\mathrm{x}-\mathrm{y} \sin \theta
$$

and

$$
\mathrm{y}^{\prime}=\mathrm{x} \sin \theta+\mathrm{y}
$$

If we take the match residuals using Equations 4.5 and 4.6, we would expect to see the match residual in $\mathrm{X}(\mathrm{Y})$ have a dependence on $\mathrm{Y}(\mathrm{X})$ as stated above. We plot this for data and the simulation in Figure 4.13. In data, we find a 1 milliradian rotation in the $\mathrm{X}$ coordinate and 2 milliradian rotation in the $\mathrm{Y}$ coordinate suggesting a fairly small rotation is present. This rotational offset is small enough that a correction is not applied.

\subsubsection{Mapping Electronics Channels to Detector Channels}

Electronics channels on a particular FEB need to be associated with a particular detector channel. This involves a complex mapping that requires detailed knowledge of fiber connections for all detector channels and the weave of fibers within a given PMT. A new hit is created for each unique signal associated with a particular detector strip. 

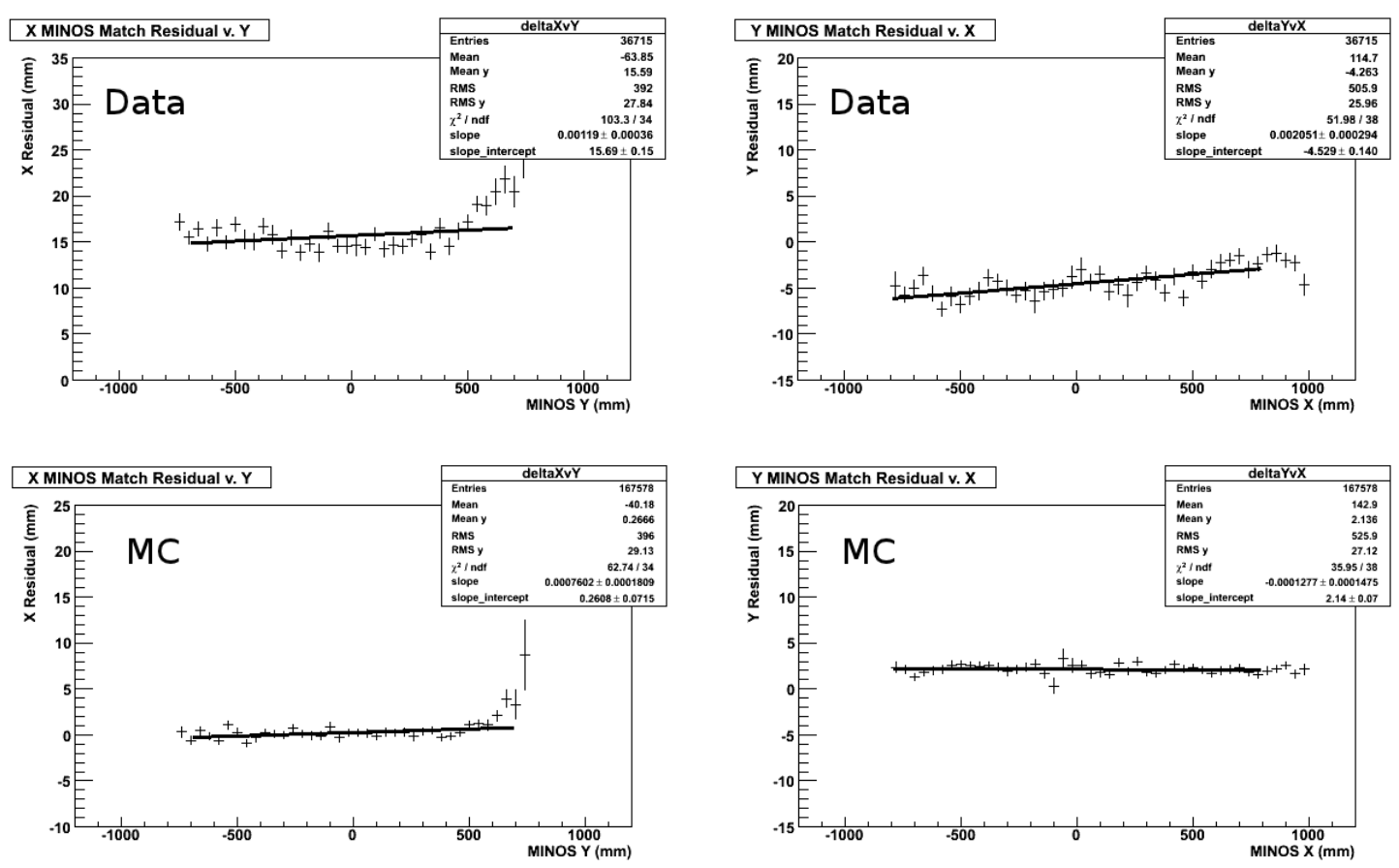

Figure 4.13: Plots searching for rotations in the XY plane between the MINER $\nu \mathrm{A}$ and MINOS detectors in data and Monte Carlo (MC). If a rotation exists, one would expect the match residual in the $\mathrm{X}(\mathrm{Y})$ coordinate to scale with $\mathrm{Y}(\mathrm{X})$ coordinate. This should show up as a slope in both plots. We observe evidence for rotations of 2 milliradians and 1 milliradian in data. Since the potential rotation is small, no correction is made for this.

\subsubsection{Information from the NuMI Beam}

As a last step of low level processing we match information gathered on each NuMI spill to each MINER $\nu \mathrm{A}$ gate. We access this information from a MINOS mysql database. This information includes the number of POT for that particular spill and various beam parameters such as the transverse width of the beam, the current on the horns, and the time of the spill. POT are the quantity in which we report the total beam exposure. 


\subsection{MINER $\nu \mathrm{A}$ Monte Carlo}

The simulation for our analysis involves several different Monte Carlo (MC) packages. We use the GENIE [89] package, version 2.6.2, to simulate neutrino interactions and final state interactions within the target nucleus. We use different Geant packages, Geant4 and GEANT3, to model several different systems. Geant4 is a $\mathrm{C}++$ based, object oriented simulation package used to simulate the basic physical interactions of particles in matter [90]. The package allows flexible usage of interaction models and also allows tuning of some of those models. Geant4 is an integral part of the G4numi package that we use to simulate the neutrino and anti-neutrino fluxes. G4numi uses Geant4 version v9.2p03. Geant4 version 9.4.p02 is used to simulate particle interactions inside MINER $\nu \mathrm{A}$ as well as the behavior of different detector components such as the electronics. We use Geant4 version 9.3 to simulate the effect of the ArgoNeuT detector on reconstruction. An earlier Fortran version of Geant, GEANT3, version 21.14.a, is used to simulate the MINOS detector [91].

\subsubsection{Simulation of the NuMI Beam}

We simulate the target and horn systems to predict the spectrum of pions and kaons that create the neutrinos and anti-neutrinos that we observe in the MINER $\nu \mathrm{A}$ detector. The g4numi package is used for this purpose which makes use of QGSP physics lists in Geant4 9.2p03 [90] to model hadron production and reinteractions within the target system. This model is imperfect, which is why we apply a reweighting scheme to better reflect the flux. Reweighting involves applying a multiplicative factor for particular events when populating distributions based on the weight a particular event should have. We describe this method in Section 4.2.2. This reweighting 
scheme cannot be applied for all simulated neutrino interactions. For those cases, we simulate the flux with other hadron models, namely QGSP_BERT, QGS_BIC, QGSC_BERT, FTFP_BERT, and FTF_BIC. We use the resulting spread of predictions in the "1000 universes" method described in the Section 5.3.5 on systematic errors stemming from the flux. In all cases, we simulate the decay of pions and kaons in flight to muons, neutrinos, and anti-neutrinos.

\subsubsection{Central Value Reweighting}

The g4numi simulation predicts pion and kaon production off of the graphite NuMI target; however, issues exist with the accuracy of the predicted pion and kaon production cross-sections. To address this, two exterior data sets are used to reweight the predicted pion and kaon distributions created by $120 \mathrm{GeV}$ protons colliding with the NuMI target. For pions with $\mathrm{x}_{\mathrm{F}}<0.5$, NA49 data [92] is used to reweight pion production cross-sections, where $\mathrm{x}_{\mathrm{F}}$ is Feynman $\mathrm{x}$ defined [5]

$$
\mathrm{x}_{\mathrm{F}} \approx \frac{2 \mathrm{p}_{\mathrm{L}}}{\sqrt{\mathrm{s}}}
$$

with $\mathrm{p}_{\mathrm{L}}$ the longitudinal momentum and the Mandelstam variable $\mathrm{s}$ is the square of the center of mass energy. The NA49 experiment, with the more verbose description, Large Acceptance Hadron Detector for an Investigation of $\mathrm{Pb}$-induced Reactions at the CERN SPS, studied pion production from proton-carbon collisions for protons with $158 \mathrm{GeV} / \mathrm{c}$ of momentum. For $\mathrm{x}_{\mathrm{F}}>0.5$, both data from NA49 and Barton, et al. [93] is used to reweight the sample. Producton cross-sections for kaons are also reweighted using NA49 data, but only for $\mathrm{x}_{\mathrm{F}}<0.2$. For all other events, no reweighting is applied. 
For regions of $\mathrm{x}_{\mathrm{F}}$ where we apply reweighting, we do so based on values of $\mathrm{x}_{\mathrm{F}}$ and $\mathrm{p}_{\mathrm{T}}$, where $\mathrm{p}_{\mathrm{T}}$ is the transverse momentum. We compare a simulated crosssection with the one measured measured in the Barton and NA49 data as a function of $\mathrm{x}_{\mathrm{F}}$ and $\mathrm{p}_{\mathrm{T}}$. We then reweight events based upon the differences of the crosssection in data and simulation. We show reweighted flux plots using this method in Figure 4.14.
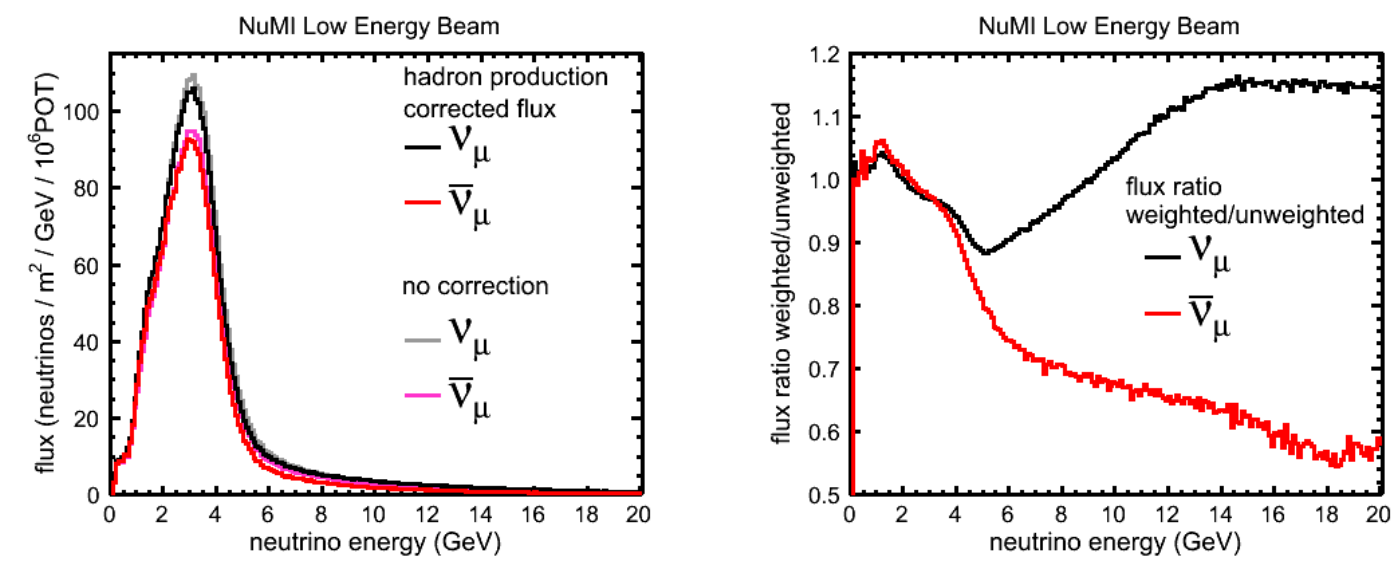

Figure 4.14: Overlay and ratio plots of the simulated flux before and after the central value reweighting procedure has been applied. The label $\nu_{\mu}$ refers to the distribution of $\nu_{\mu}$ 's in the FHC beam while the label $\bar{\nu}_{\mu}$ refers to $\bar{\nu}_{\mu}$ 's in the RHC beam.. These plots are courtesy of L. Aliaga of the MINER $\nu$ A colaboration.

\subsubsection{The GENIE Simulation}

GENIE is a $\mathrm{C}++$ and ROOT [94] based simulation package created for modeling neutrino interactions in matter. We use GENIE version 2.6.2 for this analysis. Various interaction models are used for predicting the cross-section for different types of processes. We use the formalism derived by Lewellyn Smith, which is discussed in Section 1.3.1, to model the CCQE cross-section. We use the dipole approximation for the axial form factor discussed in Section 1.3.5 and given in Equation 1.21. 
This form is motivated by assuming an exponential distribution of weak charge, the Fourier transform of which has a dipole form. The simulation uses a value of $\mathrm{M}_{\mathrm{A}}$ of $0.99 \mathrm{GeV}$, which is similar to values found by many previous experiments [45]. Here, $\mathrm{g}_{\mathrm{A}}=-1.267$, which we also discuss in Section 1.3.5. We use electromagnetic form factors outlined by R. Bradford, A. Bodek, H. Budd and J. Arrington [95], which are commonly refered to as BBBA form factors. The psuedo-scalar form factors are found using the PCAC also discussed in Section 1.3.4.

Backgrounds are described by several models. The Rein-Seghal model [96] describes resonant pion production, which is the biggest background for the analysis presented in this dissertation. Deep inelastic scattering is simulated using the Bodek-Yang model [97]. Neutral current scattering is modeled based on work by R. E. Hendrik and L. Li [98].

Although some interactions in this analysis are on free protons, most interactions are on nucleons contained within a carbon nucleus. We model that nucleus uisng the Relativistic Fermi Gas (RFG) Model which is discussed in Section 1.3.6. Nucleons have a high momentum tail described by Bodek and Ritchie [51]. The RFGM enforces Pauli blocking by requiring that a nucleon involved in an interaction contain more than the Fermi Momentum in the RFGM, or $221 \mathrm{MeV} / \mathrm{c}$ for the case of carbon. Final state effects are also modeled, which involves interactions of the final state nucleon as it exits the nucleus. GENIE uses the INTRANUKE package for this purpose.

We generate events using the GENIE simulation by acquiring an energy and neutrino flavor from the beam simulation. Using a random number seed, we then generate a particular interaction based upon that random number seed and the predicted likelihood for the different interactions. 


\subsubsection{Simulating MINER $\nu \mathrm{A}$}

The simulation in MINER $\nu \mathrm{A}$ involves several steps. In the first step, we use Geant4 to simulate the behavior of the final state particles predicted by the GENIE simulation. This involves simulating the amount of energy deposited in each step for a given particle as well as modeling any multiple scattering or secondary interactions that may occur.

The second step of the simulation involves modeling the actual detector components. The energy deposits predicted by Geant 4 are converted into a prediction of the number of photons generated in the scintillator. This includes accounting for Birks' Law which describes how the light yield from energy deposited in scintillator saturates for larger energy deposits [99]. The capture of light by the WLS fiber and its path to the PMT are also simulated. We model the quantum efficiency of the anode on the PMT, which converts a percentage of light from the WLS fiber into electrons that can be accelerated in each stage of the PMTs. We simulate the first two dynode stages of the PMT. The remaining 10 stages are modeled with a Gaussian distribution the mean of which is the average gain for a single stage of the PMT. A simulation of the electronics is also put into practice. This yields simulated hits that can be processed in the same way as hits in data.

\subsubsection{The ArgoNeuT Simulation}

We describe ArgoNeuT in Section 3.9. Due to the large amount of material comprising ArgoNeuT, it is necessary to simulate it to ensure that energy loss within that volume is properly accounted for. A simulation of the ArgoNeuT detector was created using a conversion of an AutoCAD [100] depiction of the aparatus to 
GDML [101]. We run the resulting GDML file in a standalone Geant4 simulation. This simulation is composed of thousands of tesselated pieces that approximate the shape of the original AutoCAD rendering. For simplicity, two materials were used for this simulation, liquid argon inside of the cryogenic container and stainless steel for the remainder of the components.

To simulate the effects of ArgoNeuT on our reconstruction, the exiting fourmomentum and position of muons were recorded if they exit the simulated MINER $\nu \mathrm{A}$ detector. Each muon is then inserted into the appropriate place within the ArgoNeuT simulation. If the muon is not stopped within ArgoNeuT, the exiting fourmomentum and position is recorded so the event may be simulated inside of MINOS. The predicted energy loss of particles traversing ArgoNeuT based on this simulation is shown in Figure 4.15. The region of greatest predicted energy loss coincides with the liquid argon portion of the ArgoNeuT detector. The supporting steel structure also contributes to the predicted energy loss.

\subsubsection{The MINOS Simulation}

Muons that pass out of the back of MINER $\nu \mathrm{A}$ and are not found to stop in ArgoNeuT are passed to the MINOS simulation. This is done by passing the position of entry, the type of particle, and the four-momentum of that particle to the MINOS simulation. The MINOS simulation uses GEANT3 to model the MINOS detector and the magnetic field present within that detector. This simulation runs separately from the MINER $\nu \mathrm{A}$ simulation as well as the MINER $\nu \mathrm{A}$ software framework. 


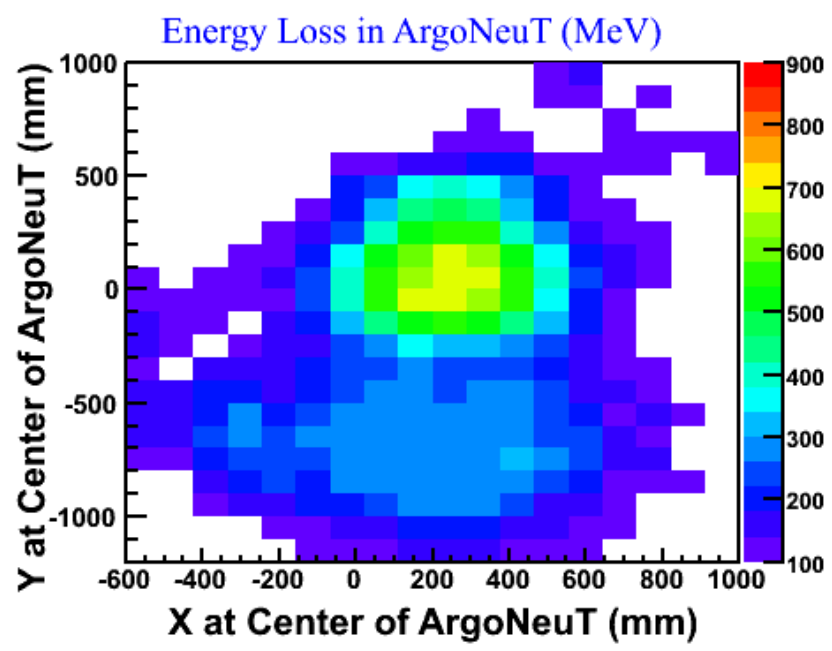

Figure 4.15: The predicted energy loss of muons passing through ArgoNeuT in units of $\mathrm{MeV}$. The positions on the $\mathrm{X}$ and $\mathrm{Y}$ axes are the projection of the muon to the center of the ArgoNeuT detector in the $\mathrm{Z}$ direction. The area of highest energy loss is consistent with the cryogenic volume containing liquid argon.

\subsection{High Level Data Processing}

The same high level processing steps are employed for data and the simulation. Several steps of processing occur. These are outlined below.

\subsubsection{Matching MINOS and MINER $\nu$ A Data Sets}

One of the earliest stages of high level processing is combining MINOS and MINER $\nu \mathrm{A}$ detector information. This includes associating MINER $\nu$ A gates and MINOS snarls with each other. A MINOS snarl is the analog of a MINER $\nu$ A gate. In the case of data, gates and snarls are matched using GPS timing information. In the case of the simulation, a given MINER $\nu \mathrm{A}$ gate and MINOS snarl are known to be associated $a$ priori.

For a given MINOS snarl, we run the full MINOS reconstruction prior to gate 
and snarl matching. This reconstruction includes a MINOS track finding algorithm, which uses a Kalman Filter and accounts for multiple scattering and deflection due to the magnetic field.

\subsubsection{Forming Time Slices}

Given the fine timing resolution of MINER $\nu \mathrm{A}$ and the timing calibrations that we apply, most neutrino interactions have a narrow time profile. Since multiple interactions can occur within the detector, separating interactions by their time offers an effective way of disentangling interactions that would otherwise overlap in space. To do this, hit times are sorted using a Heap Sort [102], which is known to be an efficient method [103].

Once hits are sorted, a sweep begins to find concentrations of hits in time. If at least two hits with a minimum amount of charge associated with them are found within a particular window, the hits are grouped together as a time slice. Hits are then added to this time slice if they occurred close to the initial hits in the time slice. The length of time used for this window is driven by the integration time in the FEBs.

In this step, only hits that pass the discriminator threshold are used. After all such hits are considered, the remaining hits are then matched to time slices if they fall within the spread of times for a particular time slice. The time slice is then used as the basic unit in processing for the remainder of reconstruction. Figure 4.16 depicts all hits in a particular gate separated into time slices. 


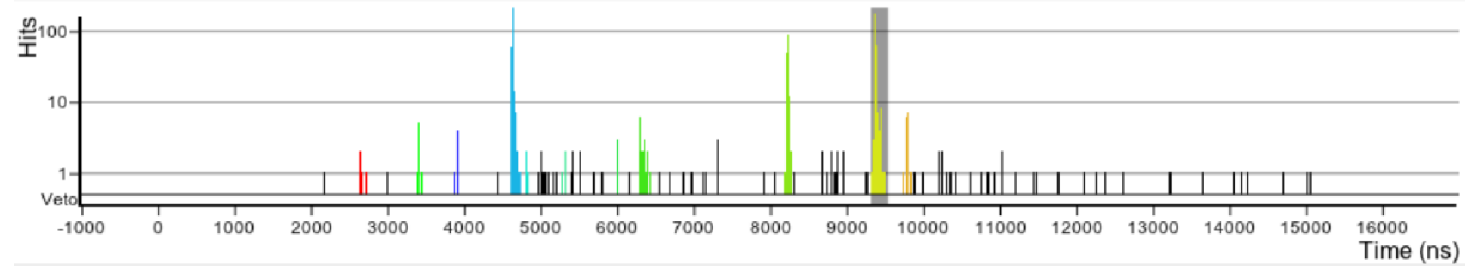

Figure 4.16: Histogram of hits as a function of time for a particular gate in data after it has been separated into time slices. The histogram comes from the Arachne event display [104]. Different time slices are represented by different colors while unused hits are depicted in black. Note that different peaks are correclty identified as unique time slices.

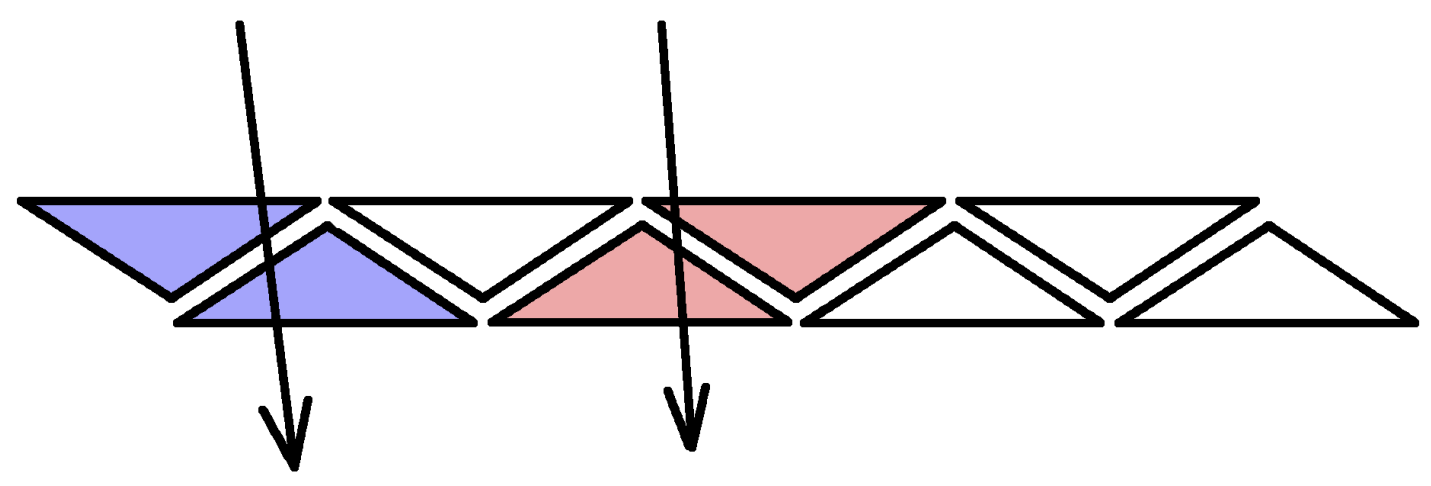

Figure 4.17: An illustration of clusters formed from MINER $\nu$ A strips within a plane. Particles traversing the plane are depicted as black arrows. The strips that are traversed by the arrows are colored light blue and pink, which are intended to represent unique clusters. Two clusters are formed since a strip without a hit bisects them.

\subsubsection{Forming Clusters}

To form clusters, we look for groupings of hits directly adjacent to each other within a plane within a given time slice. Any space between hits leads to a new cluster being formed, where a space is a strip that did not register a hit. An isolated strip without neighbors that registered a hit is also promoted to a cluster. An illustration of clusters being formed from strips is shown in Figure 4.17.

A position is calculated for each cluster. The energy on each strip is considered and the energy-weighted position is then calculated using all hits contained within 
a cluster. A time is also found for a cluster, where the time from the hit with the most energy within the cluster is asigned as the cluster time.

We classify the resulting clusters by their composition. We identify clusters as either low activity clusters, trackable clusters, heavy ioning clusters, superclusters, or cross-talk clusters. Clusters with less than $1 \mathrm{MeV}$ are considered low activity clusters. The following requirements must be met for a cluster to be considered trackable.

- $1 \mathrm{MeV} \leq$ Total cluster Energy $<12 \mathrm{MeV}$

$-\leq 4$ hits

- Must have either one or two hits with hit Energy $\geq 0.5 \mathrm{MeV}$

- If two hits are present with hit Energy $\geq 0.5 \mathrm{MeV}$, they must be directly adjacent to each other

For a cluster to be considered a heavy ionizing cluster, it must meet similar criteria to trackable clusters.

- Total cluster Energy $\geq 1 \mathrm{MeV}$

- $\leq 4$ hits

- Must have either one, two, or threes hits each with hit energy $\geq 0.5 \mathrm{MeV}$

- If two or threes hits are present with hit Energy $\geq 0.5 \mathrm{MeV}$, they must all be directly adjacent to each other

- Must not qualify as a trackable cluster 
Heavy ionizing clusters are important in forming high angle tracks. Any cluster with more than $1 \mathrm{MeV}$ in energy that does not meet the criteria for either trackable or heavy ionizing clusters is classified as a supercluster. Any cluster with five or more hits is automatically considered a supercluster.

A cluster is identified as a cross-talk cluster by inspecting the PMT pixels associated with hits within that cluster. The PMT pixels associated with that cluster are compared to PMT pixels associated with a particle interaction. If these cluster PMT pixels are found to be directly adjacent to the pixels related to the particle interaction, the cluster is considered to be a cross-talk cluster.

\subsubsection{Tracking}

Photons and charged particles generally deposit energy in each MINER $\nu$ A plane they traverse. We identify these deposits and use them to reconstruct the particle trajectory. We refer to the resulting reconstructed object as a track.

The strategy we use for reconstructing tracks within a time slice involves first finding as many tracks as possible and out of those tracks identifying a muon track associated with a neutrino interaction. All other tracks are then deleted. Once the muon track has been identified, it can be further refined by picking up activity that may have been missed in the initial pass of tracking. The muon is identified first since it is one of the easiest particles in an interaction to find, removing activity associated with a muon generally improves the overall success rate of finding other tracks, and the start of the muon track is generally the vertex for the neutrino interaction that created the muon. After finding the muon we then search for any hadron tracks using the same tracking procedure we used to find the muon. We then attempt to connect events with a common vertex and then refit them using a 
Kalman filter $[105,106]$. A search is then made for any other tracks from coincident activity within the event using the same tracking procedure as before. A description of the tracking algorithm used in each step follows below.

\section{Making Track Seeds and Track Candidates}

The first step in the tracking process is making two dimensional track seeds. To make these seeds, we sort trackable and heavy ionizing clusters by their location along the Z-axis. Track seeds are then found by looking for clusters within planes with the same view for three clusters that are in a line. A determination of linearity is made based on doing a least squares fit to a line and enforcing a minimum $\chi^{2}$ value for the fit. Only tracks seeds that pass that linearity cut are considered in the next step of tracking. This process is illustrated in Figure 4.18.

Once we have created a set of track seeds, we combine track seeds into track candidates. We initiate this merging process in the downstream end of the detector and work upstream. This order is used since muons will generally traverse a large fraction of the detector and consequently are likely to be found by looking for tracks in the downstream end of the detector. Employing the reverse of this method by finding the vertex of the muon track and initiating the track from that point is difficult since high amounts of activity can be present near the vertex.

We combine track seeds by looking for track seeds that share a cluster and then comparing slopes of each. If the slopes are similar enough, the track seeds are combined into a track candidate. The result is two dimensional track candidates each composed of clusters in a single view. We then attempt to merge recently formed track candidates comparing the slope and slope intercepts of each. If the two are similar enough, we combine them into a single track candidate. When 


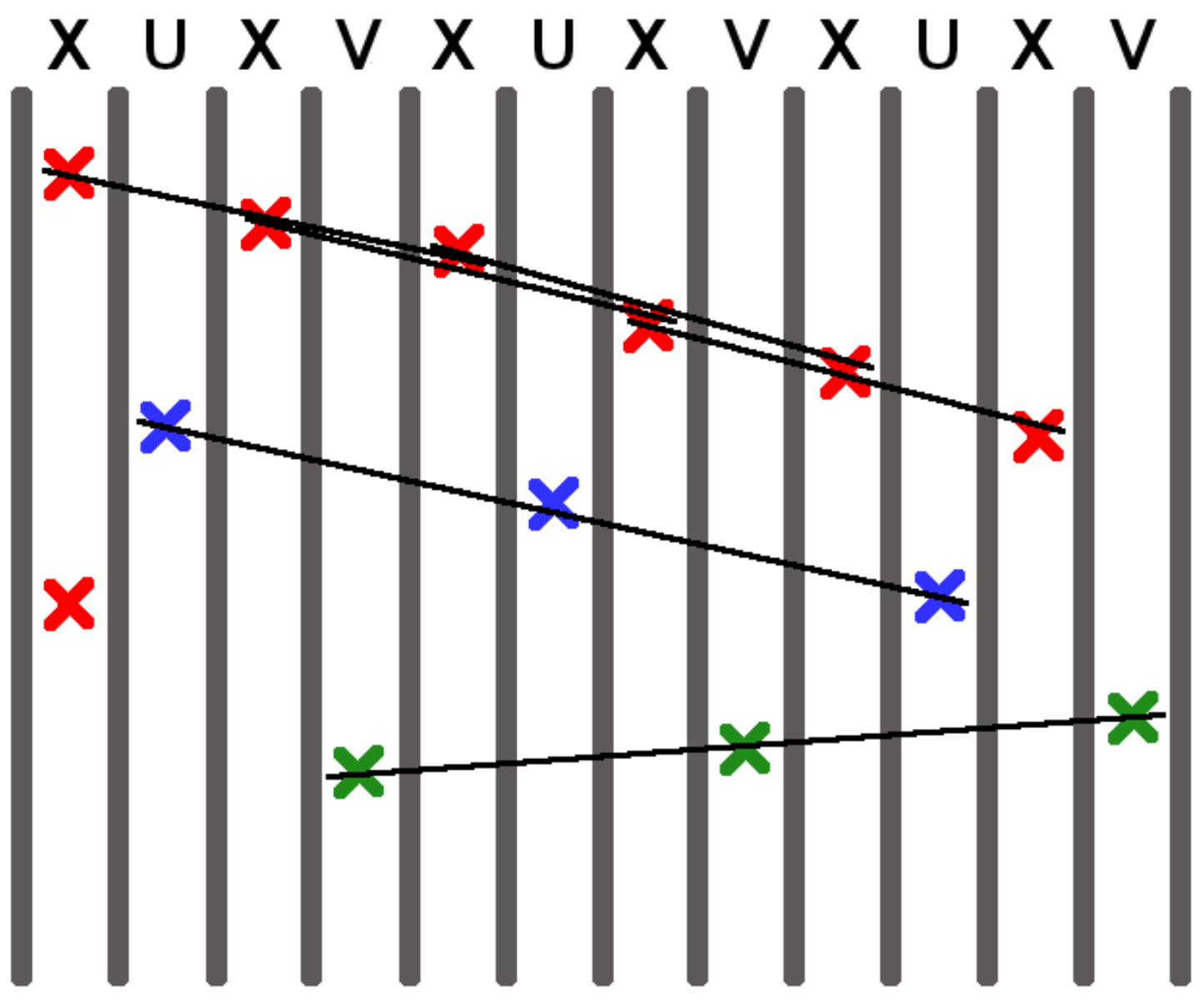

Figure 4.18: An illustration of track seed formation. Clusters are marked by a 'X' with a color that varies by plane type. Clusters within a particular view are formed into three cluster seeds. In cases where multiple tracking seeds are made, particular seeds may overlap. Some clusters cannot be used to successfully construct a track seed, such as one of the hits in the first X plane. 


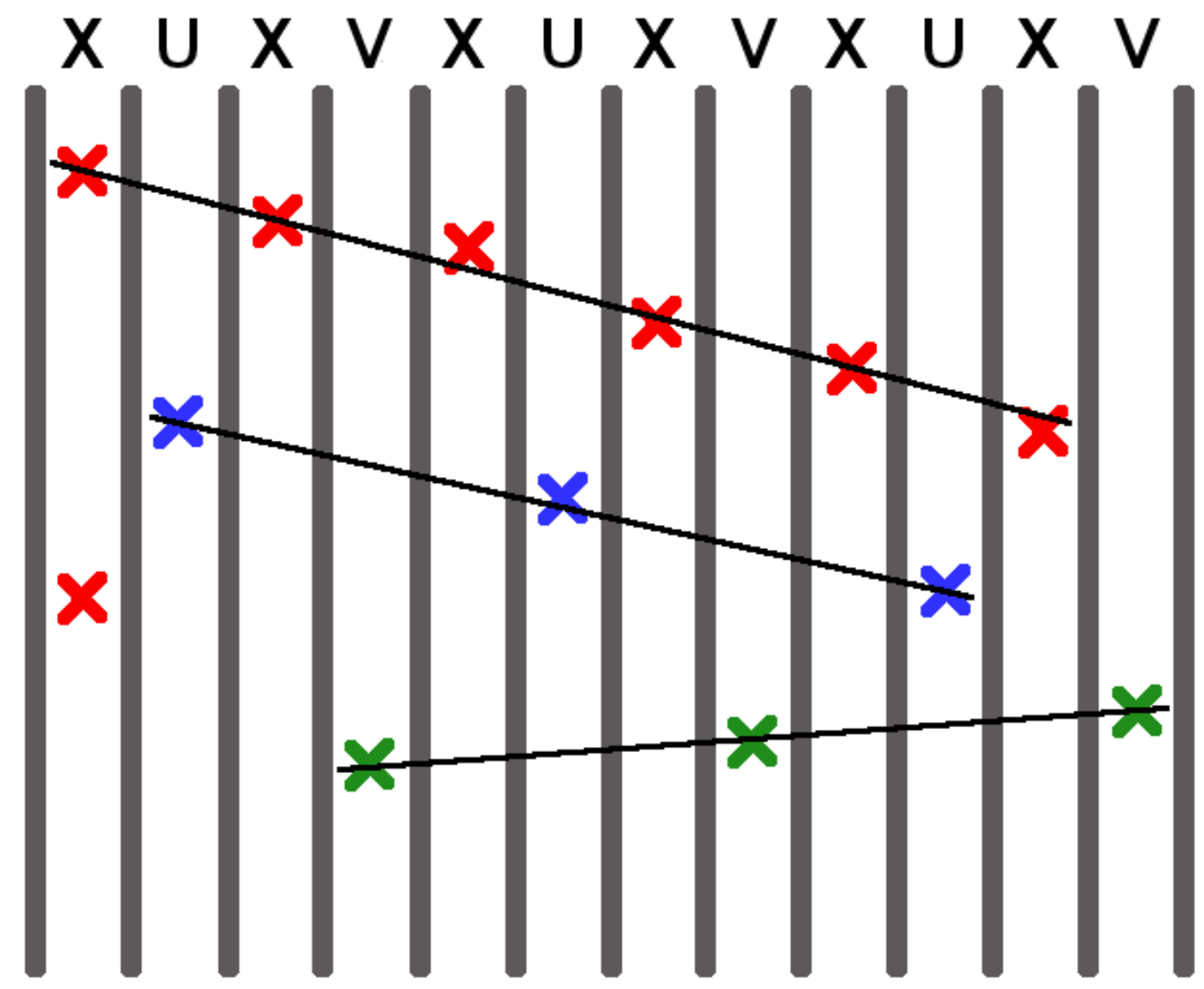

Figure 4.19: An illustration of track candidate formation. In the pictured scenario, track seeds in the $\mathrm{U}$ and $\mathrm{V}$ planes are promoted to track candidates. The track seeds in the $\mathrm{X}$ view are merged into a single track candidate.

creating track candidates, we enforce that a track candidate contain no more than a single cluster per plane. The result is a number of track candidates each composed solely of clusters from a single detector view. An illustration of this is shown in Figure 4.19.

The next step of tracking involves taking two dimensional track candidates from different views and attempting to combine them into a three dimensional track. We first enforce that the tracks are colinear by cutting on the $\chi^{2}$ value for a least squares 
fit of the three dimensional track to a straight line. We also check the amount of overlap in the Z-direction. If the track passes the $\chi^{2}$ cut and sufficient overlap is found, the track candidates are combined into a track object.

We next check for tracks that have a kink. Occasionally, a particle can undergo a hard scatter resulting in a kink in two of the views while still appearing straight in the third view. In this case, overlap is inspected on each side of the kink separately. The two pieces on either side of the kink can then be formed into a single track object.

\section{Kalman Filter}

We fit a track using a Kalman filter implementation that incorporates multiple scattering $[105,106]$. The Kalman filter is a recursive method that takes input data to make a statistically optimized prediction assuming a linear system which is then used as an input for the next iteration of the filter.

The multiple scattering calculation incorporates material information for the region of the detector the track is traversing as well as the mass of the particle. For the first round of tracking where we search for a muon track, we use the mass of the muon in calculating multiple scattering.

\section{Using Track Projections to Extend and Populate Tracks}

We use the fit results to further extend and populate a track. This includes extending a track upstream and downstream as well as filling in any empty planes within the track object. For this procedure, we consider not just trackable and high ionizing clusters, but also low activity clusters and superclusters. For a particular cluster, we use the result of the Kalman filter fit for the location nearest the cluster in question. 
This gives local slope information and allows proper handling of multiple scattering. If this projection passes through any part of the cluster object, we prepare to add it the track. If a cluster is a low activity, trackable, or high ionizing cluster, we simply add it to the track. If the cluster is a supercluster, we break the supercluster into at least two pieces. One cluster consistent with a energy deposit from a minimum ionizing particle (MIP) in the path of the track is created. This cluster will have $2.25 \mathrm{MeV} / \mathrm{cm}$ of energy where the pathlength of the particle through a strip is considered in determining how much energy to allocate. The supercluster has the energy used to create the cluster for the track deducted from the relevant strips within the cluster. Note that if less than a MIP worth of energy is available, all energy in the path of the track is used to make the cluster that will be added to the track. In some cases, the supercluster is broken into three components one of which is added to a track.

This method accomplishes several important tasks. Filling in gaps on tracks helps ensure that energy that is properly associated with the track does not end up in the recoil energy calculation we make in Section 5.1.3. The method ensures that the track is extended back to the true vertex of the neutrino interaction. The method also ensures the track is extended to the downstream end of the detector. This aids in correctly matching the track to a track in MINOS.

\section{Track Cleanup}

After the above step of filling in gaps in tracks where possible, we inspect each track to determine if the object ought to be split into two pieces. Looser standards regarding gaps along a track are initially used since some activity related to the track may be part of a supercluster and thus not available for forming a track. If no 
activity is found to fill in the empty spaces along a track, we find some tracks have large gaps of planes separating the upstream and downstream ends. If this gap is too great, we split the track into two separate pieces, considering them to be caused by separate particle interactions.

\section{Applying the Tracking Algorithm}

As mentioned above, after reconstructing the muon track we search for hadronic particles. The track we consider most likely to be the muon is saved and all other tracks deleted. We then use the muon track as an anchor to find hadronic particles with the same vertex. We take the remaining trackable and high ionizing clusters and repeat the above tracking algorithm with the requirement that tracks must have a common vertex with the muon.

A Kalman filter fit is done to the track and vertex system. Any tracks that do not have a vertex consistent with the muon track are deleted. Track cleanup is peformed on the newly created tracks splitting them if large gaps exist within the track. We repeat this anchor-based tracking using the ends of all tracks as potential new vertices. We use the same tracking and anchoring method used for the muon track. After this search for secondary vertices, we search for tracks not associated with the reconstructed muon track. All remaining trackable and high ionizing clusters are used. Again, the same track procdure before is used, with a final cleanup step of splitting newly found tracks if appropriate.

\subsubsection{Attenuation Correction}

The size of the observed signal within a strip given a specific energy deposit by a traversing particle depends on the position along the strip that the particle inter- 
acted. The reason for this position dependence is the attenuation of light within the WLS fiber. Given a single hit within a strip and no other information, a correction for this attenuation cannot be made since there is no way to know where along the strip the hit occured. However, once tracks have been created, we know the three dimensional position of the various clusters that compose the track. Given the three dimensional information, we can account for the attenuation of signals that traveled longer or shorter pathlengths. In earlier stages of processing, we calibrate cluster energy as if each one is in the center of the strip. For clusters where we have three dimensional information available, we calculate and apply a correction for how the signal differs from a hit that happens at the ceneter of the strip in the longitudinal direction.

\subsubsection{Incorporating MINOS Reconstruction}

We attempt to match MINER $\nu \mathrm{A}$ tracks that have a cluster in at least one of the last five modules in the detector to tracks in MINOS. The resulting object we refer to as a prong. To qualify as a match, the MINOS track must start within the first

four planes of MINOS. We only consider matching MINOS and MINER $\nu$ A tracks if they are within 200 ns of each other. Figure 4.20 shows the time difference between all matched tracks for Frozen Detector data. To make a match, we use a track projection method. We project the MINOS track across the two meters separating the two detectors to the plane that contains the last cluster on a MINER $\nu \mathrm{A}$ track and we project the MINER $\nu$ A track to the plane that contains the vertex of the MINOS track. For MINER $\nu$ A, we compare the position of the last cluster on a track with the projection of the MINOS track. We refer to the distance between these two points as the match residual. We also look at the analagous quantity in 
MINOS, where we find the residual of the projection from MINER $\nu \mathrm{A}$ and the vertex of the MINOS track. We look at the size of the match residuals at both faces to assess whether the two tracks are likely to have been created by the same particle. If both match residuals are less than $40 \mathrm{~cm}$, we consider them to be matching tracks. If multiple potential matches exist, we take the match with the smallest match residual.
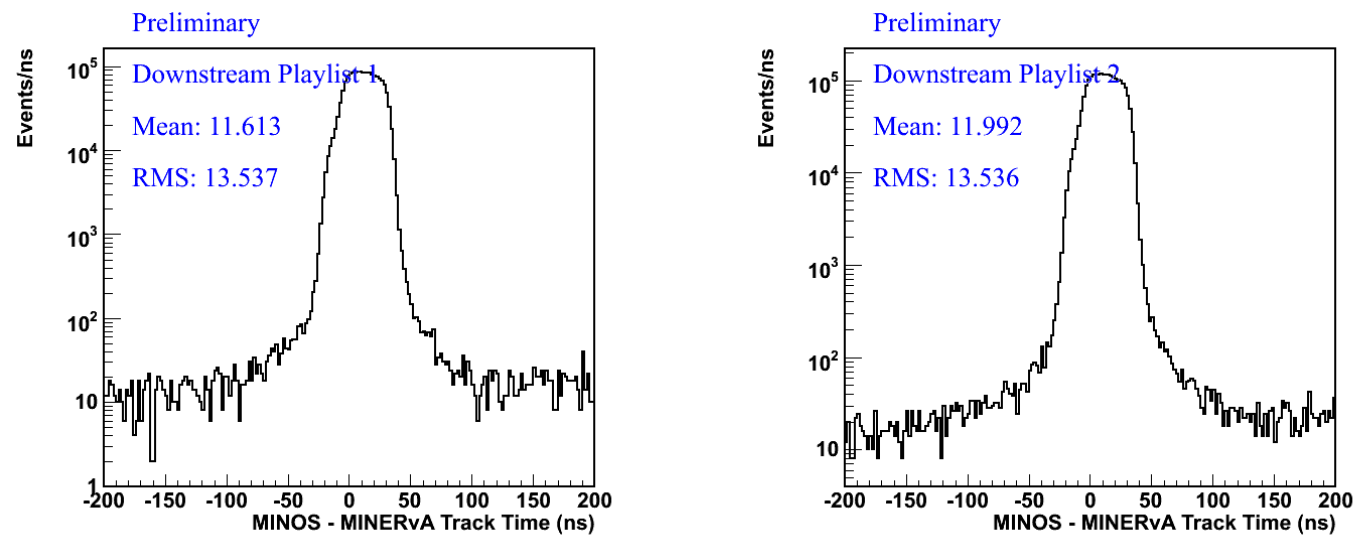

Figure 4.20: Plots of the time difference between matching MINOS and MINER $\nu \mathrm{A}$ tracks for the Frozen Detector data sets. The data set labeled as Downstream Playlist 1 is for the period where no construction was occurring and Downstream Playlist 2 is for data after the resumption of construction. Most events are sharply peaked close to 13 ns. Note that no correction is made for the time of flight of the muons.

If no matches have been found for a MINER $\nu \mathrm{A}$ track, we also attempt matching using a closest approach method. We project the MINOS track toward MINER $\nu \mathrm{A}$ and the MINER $\nu \mathrm{A}$ track toward MINOS and look for the two tracks to cross close to a common point in space. This method can be useful for the case where ArgoNeuT is present between the two detectors. In that instance, a muon could undergo a hard scatter within ArgoNeuT and still potentially get matched. Figure 4.21 shows both match residuals and the result of the closest approach method.

The MINOS track has a charge associated with it which is found by examining 


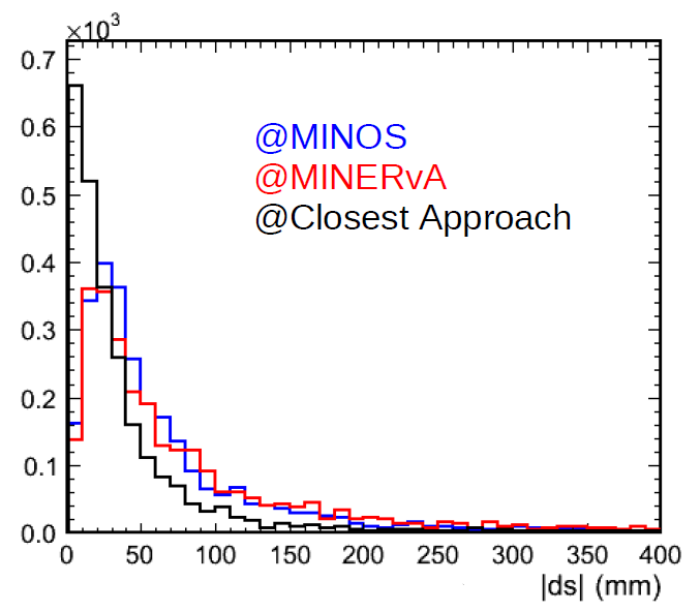

Figure 4.21: The match residual at the MINOS and MINER $\nu \mathrm{A}$ faces as well as the value for the closest approach method for matching tracks. This plot was made by M. Kordosky of the MINER $\nu \mathrm{A}$ collaboration.

the direction of curvature in the MINOS magnetic field. We use energy found by MINOS reconstruction. Energy can be found in one of two ways in MINOS. In one case, range of the track is used to estimate its initial energy. The curvature of the track in the magnetic field is also used to make an energy measurment. We use range or curvature for energy reconstruction depending upon where the track begins or ends. This includes whether the track passes into region containing noninstrumented planes, in which case we use curvature for reconstruction. If the track is contained within a particular volume that is fully instrumented, we use range for the momentum measurement. In all other cases, we use the result of the curvature measurement.

We find that tracks matched to a MINOS track are almost exclusively muons giving a high level of confidence to our particle identification for such tracks. 


\subsubsection{Muon Energy Reconstruction}

As mentioned in Section 4.3.6, we use the MINOS reconstruction to find the energy of the muon while it is in MINOS. To find the energy of a muon at its vertex in MINER $\nu \mathrm{A}$, we must account for the energy lost by that muon as it travels in the MINER $\nu \mathrm{A}$ detector. We also account for energy lost in the first steel plane of MINOS and the steel mirror plane at the backend of MINER $\nu$ A. To account for this energy loss, we use the Bethe-Bloch formula [5] which gives the energy loss at each step,

$$
-\frac{\mathrm{dE}}{\mathrm{dx}}=\mathrm{Kz}^{2} \frac{\mathrm{Z}}{\mathrm{A}}\left[\frac{1}{2} \ln \frac{2 \mathrm{~m}_{\mathrm{e}} \mathrm{c}^{2} \beta^{2} \gamma^{2} \mathrm{~T}_{\max }}{\mathrm{I}^{2}}-\beta^{2}-\frac{\delta(\beta \gamma)}{2}\right],
$$

where $\mathrm{K}$ is a constant, $\mathrm{z}$ is the magnitude of the charge of the incident particle, $\mathrm{A}$ is the atomic mass, $\mathrm{Z}$ the atomic number, $\mathrm{I}$ is the mean excitation energy, $\mathrm{m}_{\mathrm{e}}$ is the mass of the electron, $\delta(\beta \gamma)$ is a density effect correction to ionization energy loss, $\mathrm{c}$ is the speed of light, and we use the relativistic variables $\beta$ and $\gamma \cdot \mathrm{T}_{\max }$ is the maximum amount of energy that can be imparted to an electron [5], given by

$$
\mathrm{T}_{\max }=\frac{2 \mathrm{~m}_{\mathrm{e}} \mathrm{c}^{2} \beta^{2} \gamma^{2}}{1+2 \gamma \mathrm{m}_{\mathrm{e}} / \mathrm{M}+\left(\mathrm{m}_{\mathrm{e}} / \mathrm{M}\right)^{2}},
$$

with $\mathrm{M}$ being the mass of the particle for which $\mathrm{T}_{\max }$ is being calculated. To apply this formula, we incrementally step along a track assessing the amount and types of material along it. Then starting from the downstream end of the track and using the energy found for the muon in MINOS where we account for steel between MINOS and MINER $\nu \mathrm{A}$, we find the amount energy loss expected based on the amount of material at that point. This energy is then added on to the muon. This new energy for the muon is used in the next calculation of the energy loss in the subsequent 
upstream step. This process is repeated until the energy of the muon has been reconstructed back to the vertex.

\subsection{Blob Formation}

We reconstruct three different types of blobs: vertex blobs, isolated blobs, and dispersed blobs. Blobs are composed of clusters and are constructed to capture different types of topologies.

Vertex blobs capture all cluster energy near the vertex, specifically within a 10 cm sphere around the vertex of the muon. We consider trackable clusters, high ionizing clusters, and superclusters, but not low activity or cross-talk clusters when creating this object. We use the same criteria for cross-talk clusters as Section 4.3.3.

We also construct isolated blob objects which are composed of contiguous areas of energy deposit. To find them, we look for clusters that are adjacent to each other in different views and that form a consistent three dimensional object. We consider all clusters except ones that contain less than $1 \mathrm{MeV}$ of energy, cross-talk clusters, clusters that have a time more than 25 ns away from the track time, and any cluster in the OD or HCAL regions of the detector.

Last, we construct a dispersed blob which contains all clusters that are not associated with a track or that are part of a vertex blob or isolated blob. We consider the same classes of clusters for dispersed blobs as are used for isolated blobs. 


\subsection{Reconstructing Neutrino Interactions}

Analysis of physics interactions occur at what we refer to as the prong level. In this case, we examine prong objects created from matching MINER $\nu \mathrm{A}$ and MINOS tracks. This object has clusters within the time slice associated with it. We then look at clusters very close in time to the prong time. The prong time is derived from the time of the MINER $\nu \mathrm{A}$ track that is matched to the MINOS track. This strategy is used since time slices in data can potentially contain more than a single neutrino interaction.

For these physics interactions, we calculate the energy of the incident antineutrino $\mathrm{E}_{\bar{\nu}_{\mu}}$ using Equation 4.10 and the four-momentum transfer squared $\mathrm{Q}^{2}$ using Equation 4.11. Both calculations assume Quasi-Elastic kinematics.

$$
\begin{gathered}
\mathrm{E}_{\bar{\nu}_{\mu}}=\frac{\mathrm{m}_{\mathrm{n}}^{2}-\left(\mathrm{m}_{\mathrm{p}}-\mathrm{E}_{\mathrm{b}}\right)^{2}-\mathrm{m}_{\mu}^{2}+2\left(\mathrm{~m}_{\mathrm{p}}-\mathrm{E}_{\mathrm{b}}\right) \mathrm{E}_{\mu}}{2\left(\mathrm{~m}_{\mathrm{p}}-\mathrm{E}_{\mathrm{b}}-\mathrm{E}_{\mu}+\mathrm{p}_{\mu} \cos \theta_{\mu}\right)} . \\
\mathrm{Q}^{2}=2 \mathrm{E}_{\bar{\nu}_{\mu}}\left(\mathrm{E}_{\mu}-\mathrm{p}_{\mu} \cos \theta_{\mu}\right)-\mathrm{m}_{\mu}^{2} .
\end{gathered}
$$

These calculations assume the nucleon the neutrino is scattering from is at rest. The term $\mathrm{E}_{\mathrm{b}}$ is the binding energy that needs to be overcome to separate the final state nucleon from the nucleus, which we assume to be $30 \mathrm{MeV}$ in this analysis. Equation 4.10 and 4.11 are both functions of the final state muon kinematics and no other observables. The terms $\mathrm{m}_{\mathrm{p}}, \mathrm{m}_{\mathrm{n}}$, and $\mathrm{m}_{\mu}$ are the proton, neutron, and muon mass, respectively.

Issues exist with reconstructing neutrino or anti-neutrino interactions assuming Quasi-Elastic kinematics. We know the nucleon is not at rest within a nucleus, which causes some error in reconstruction. Even larger issues can occur for cases 
where a background process mimics a Charged Current Quasi-Elastic interaction, such as in the case of MEC discussed in Section 1.3.6. 


\section{Chapter 5}

\section{Measuring the Charged Current Quasi-Elastic Differential Cross-Section $\frac{\mathrm{d} \sigma}{\mathrm{d} \mathrm{Q}^{2}}$}

We report a measurement of the muon anti-neutrino Charged Current Quasi-Elastic (CCQE) differential cross-section $\frac{\mathrm{d} \sigma}{\mathrm{dQ}^{2}}$ on polystyrene scintillator $(\mathrm{CH})$. In this chapter, we outline how we isolate our signal sample, perform background subtraction, unfold the sample, make efficiency and acceptance corrections, and normalize it to get our cross-section result. We give a full assessment of systematic errors for this result and then make comparisons to other simulation models.

\subsection{Event Selection}

Event selection is made by first isolating a sample of charged current (CC) muon antineutrino interactions. From this sample, we select CCQE events through a variety 
of cuts on final state topology. This event selection is discussed in Section 5.1.1 and Section 5.1.3.

\subsubsection{Isolating a Charged Current Sample}

$\mathrm{CC}$ neutrino and anti-neutrino interactions are distinguished by a charged lepton final state. For muon anti-neutrinos, this means we expect to find a reconstructed $\mu^{+}$in the final state. Given the large neutrino background in the beam and ongoing development of methods to distinguish muons and pions, we do not consider muons that stop within our detector for this analysis. We consider muons that exit from one of the last five modules in MINER $\nu \mathrm{A}$ and that are matched to a reconstructed muon in MINOS. Additionally, the reconstructed muon in MINOS must have a reconstructed energy and a positive reconstructed charge. Energy reconstruction can fail if too few planes are traversed to make a curvature measurement and the muon traverses a region where range cannot be used to reconstruct the energy.

The positive charge is determined by examining the curvature of the muon in MINOS. From that curvature, the value $\frac{q}{p}$, where $q$ is the charge of the muon and $\mathrm{p}$ is the momentum, can be extracted. More specifically,

$$
\frac{1}{\mathrm{r}} \propto \frac{\mathrm{q}}{\mathrm{p}}
$$

where $\mathrm{r}$ is the radius of curvature of the particle in the magnetic field, $\mathrm{q}$ is the charge of the particle, and $\mathrm{p}$ is the momentum. If that ratio is greater than 0 , we consider the event to be positively charged. This does allow for a small number of wrong sign events, a $\mu^{-}$but with a positively reconstructed charge, to enter the sample. These events are handled during background subtraction which we discuss in Section 5.2.1. 
The vertex of the muon must be within MINER $\nu$ A's fiducial volume. We select this volume to ensure that there is good separation between the muon vertex and the side ECAL and downstream ECAL regions. The fiducial volume spans modules 55 through 80 and all portions of those modules within a hexagon with an apothem of $85 \mathrm{~cm}$. This is to ensure that no events that occur in areas with lead absorbers leak into the signal sample. The upstream module in the fiducial volume is selected to allow for enough planes that simple tracking failures will not lead to rock muon events leaking into our sample. Rock muons are muons created from neutrino and anti-neutrino interactions in material, primarily the rock, upstream of the detector. Along with additional cuts to remove the rock muon background, the requirement that a muon be matched to a $\mu^{+}$in MINOS and the fiducial volume restriction returns a highly pure $\mathrm{CC}$ anti-neutrino sample. In Figure 5.1, the small non-CC component of the sample is visible.

Last, we only consider CC events with a reconstructed energy of $10 \mathrm{GeV}$ or less. Above neutrino energies of $10 \mathrm{GeV}$, statistics are poor and backgrouds larger making it harder to compete with previous experiments such as NOMAD that have results in this regime [45]. We then consider further cuts to select CCQE events. In all cases in this analysis, when reconstructed $\mathrm{Q}^{2}$ is found, we assumed CCQE kinematics as described in Equations 4.10 and 4.11 of Section 4.5. We refer to $\mathrm{Q}^{2}$ found in this way as $\mathrm{Q}_{\mathrm{QE}}^{2}$ for clarity. Distributions showing muon energy and reconstructed $\mathrm{Q}_{\mathrm{QE}}^{2}$ assuming CCQE kinematics are shown is Figure 5.1.

\subsubsection{Vetoing Rock Muon Events}

Muons consistently deposit energy in MINER $\nu \mathrm{A}$ scintillator strips allowing most rock muons entering the detector to be vetoed by requiring that an interaction have 

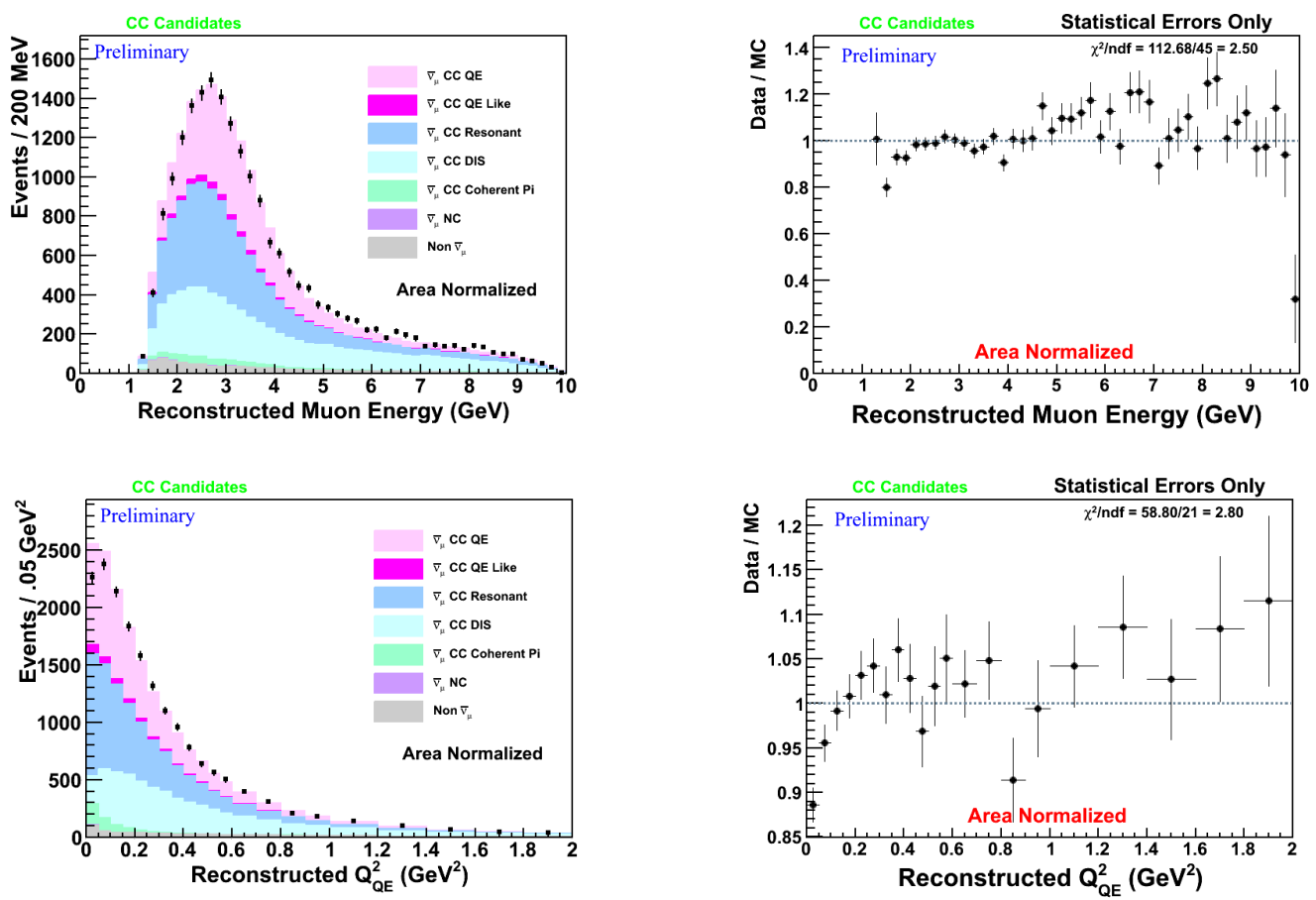

Figure 5.1: The muon energy and $\mathrm{Q}_{\mathrm{QE}}^{2}$ data/MC overlays for CC events. Data/MC ratios each with a $\chi^{2}$ value showing the consistency of the ratio with a flat line are also shown.

a vertex within the fiducial volume. However, due primarily to deadtime, rock muon events can at times mimic neutrino or anti-neutrino interactions. Deadtime is described in Section 3.7.1. When channels in the path of the muon near the boundaries of the fiducial volume are undergoing deadtime, the tracker will reconstruct a track originating from within the fiducial volume. We employ three methods to remove this background:

- Veto certain types of activity at the front of the detector

- Veto on deadtime in channels upstream of the muon track

- Scan signal event that pass both vetoes to estimate the remaining rock muon background 
To make an upstream veto, we extrapolate the muon track upstream to the two most upstream tracking modules. We then look for activity within $10 \mathrm{~cm}$ of the projection. If that activity is above a threshold of 10 photoelectrons in at least two views, we veto that event. The two view requirement limits unrelated, but coincident activity from vetoing the event. The veto on deadtime is performed by projecting the muon one and two modules upstream and examining whether the pair of TriP-t chips servicing the strips in the path of the muon underwent deadtime. If at least two instances of this are found, the event is vetoed. An example of a vetoed background event is shown in Figure 5.2.

We find both vetoes to be necessary due to results of a scan for rock muons in a portion of our signal events. Some rock muon background events fail one but not both of these vetoes. We find that the deadtime veto eliminates the greatest number of rock muon events. Although the upstream veto removes fewer rock muon events, it does remove background events not found by the deadtime veto. We also find no signal candidates to be lost by applying the upstream veto. Some signal events are lost from applying a veto on deadtime. A scan of all vetoed events allows us to measure the number of vetoed signal events. An example of such an event that is a CCQE signal candidate but is lost by making a dead time cut can be found in Figure 5.3. Figure 5.3 depicts an event in Arachne, MINER $\nu$ A's event display [104]. Even though deadtime is present in the event, clear vertex activity indicates this is an anti-neutrino interaction. We make a correction for this loss when we absolutely normalize our sample. This normalization factor is found in Table 5.5.

The scan also established the number of rock muon events remaining in the signal sample. This is discussed further in Section 5.2.1 on background subtraction. After applying all vetoes, we find the distributions found in Figure 5.1. 

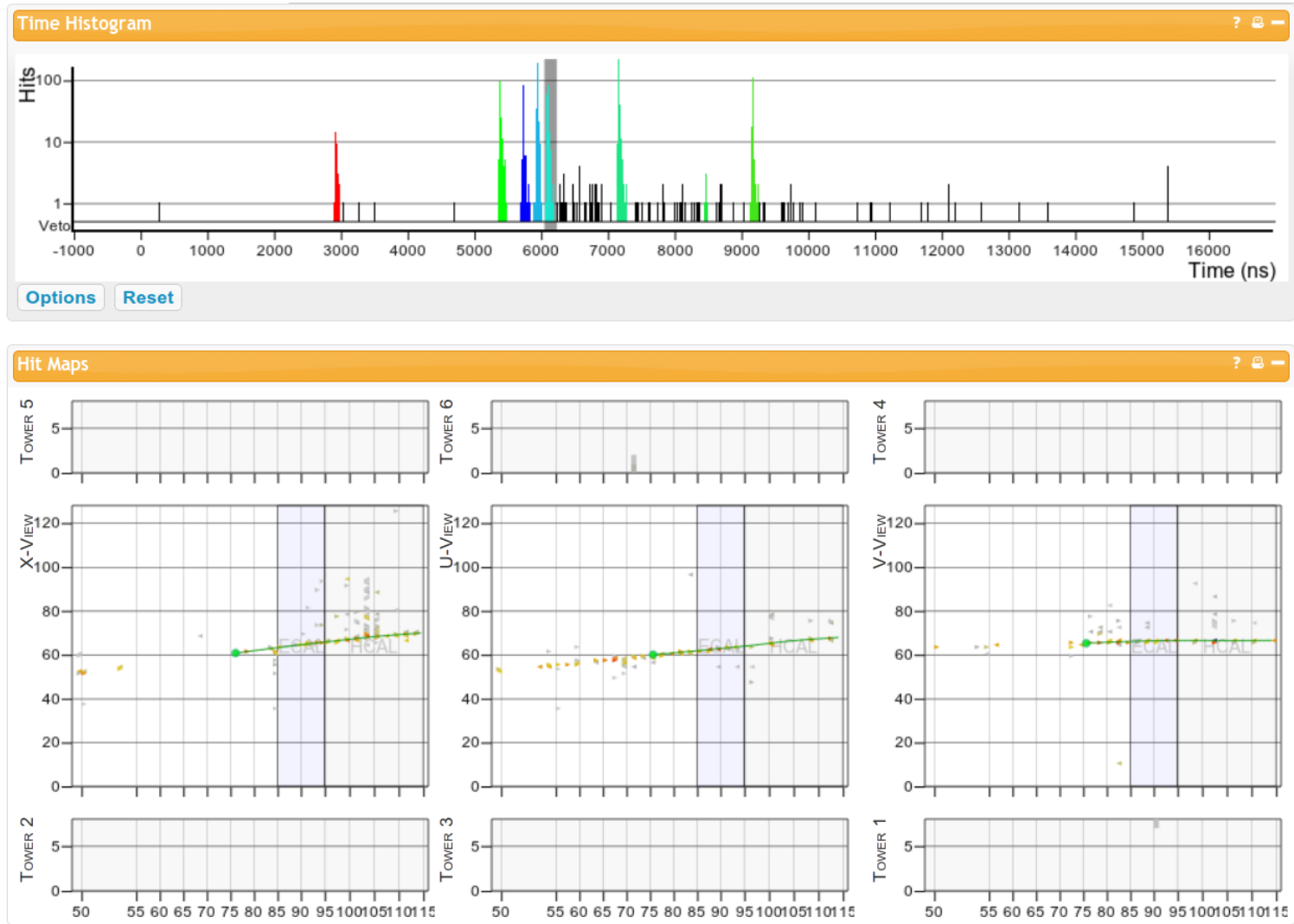

Figure 5.2: An event display of a vetoed rock muon background event in data. The time histogram shows the distribution of hits in time. The particular time slice being shown is highlighted in grey. The three different views are also visible. The reconstructed track is shown as a green line. This event is vetoed based on deadtime and upstream activity. Although the vertex is reconstructed within the fiducial volume, other activity not suppressed by deadtime clearly shows the muon to have entered from the front of the detector. Note that activity below roughtly $0.5 \mathrm{MeV}$ is not shown. 


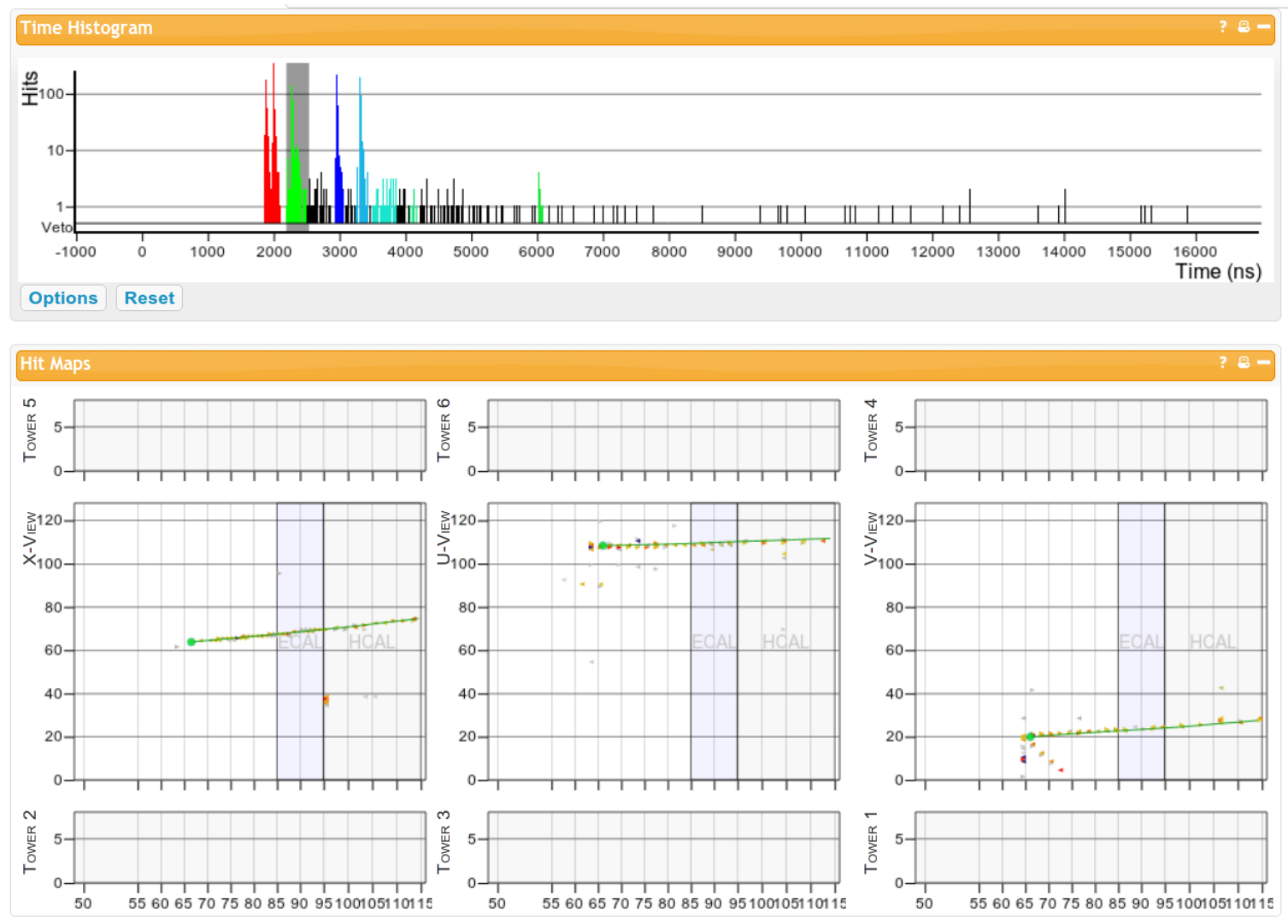

Figure 5.3: An event display of a vetoed CCQE signal candidate event in data. The time histogram shows the distribution of hits in time. The particular time slice being shown is highlighted in grey. The three different views are also visible. The reconstructed track is shown as a green line. This event is vetoed based on deadtime. Given the clear vertex activity and lack of upstream activity, this event is most likley not a rock muon. Note that activity below roughtly $0.5 \mathrm{MeV}$ is not shown.

\subsubsection{CCQE Event Selection}

Anti-neutrino CCQE events generally will have a single muon and single neutron in the final state. The neutron often does not interact before exiting the detector and when it does, will generally create a single energy deposit within the detector. It rarely forms a final state topology that could be formed into a track. Consequently, we search for a single muon track with no more than a single calorimetric energy deposit consistent with energy deposited by a neutron. Conversely, events that 
are background often have pions like in the case of resonant pion events. In other cases, such as deep inelastic scattering (DIS) events, multiple hadrons can be found in the final state. Backgrounds events also tend to have large amounts of recoil energy deposited in the detector. To find events with a CCQE final state and reject backgrounds, we make the selections outlined below.

For the following selection, we only consider activity close to the muon track in time since this final state has a narrow profile in time. For quantities on which we are making a selection, we make a cut of \pm 25 ns around the average muon track time. The average value of the muon around which the \pm 25 ns cut is made is propagated forward based on expected time of flight of the muon.
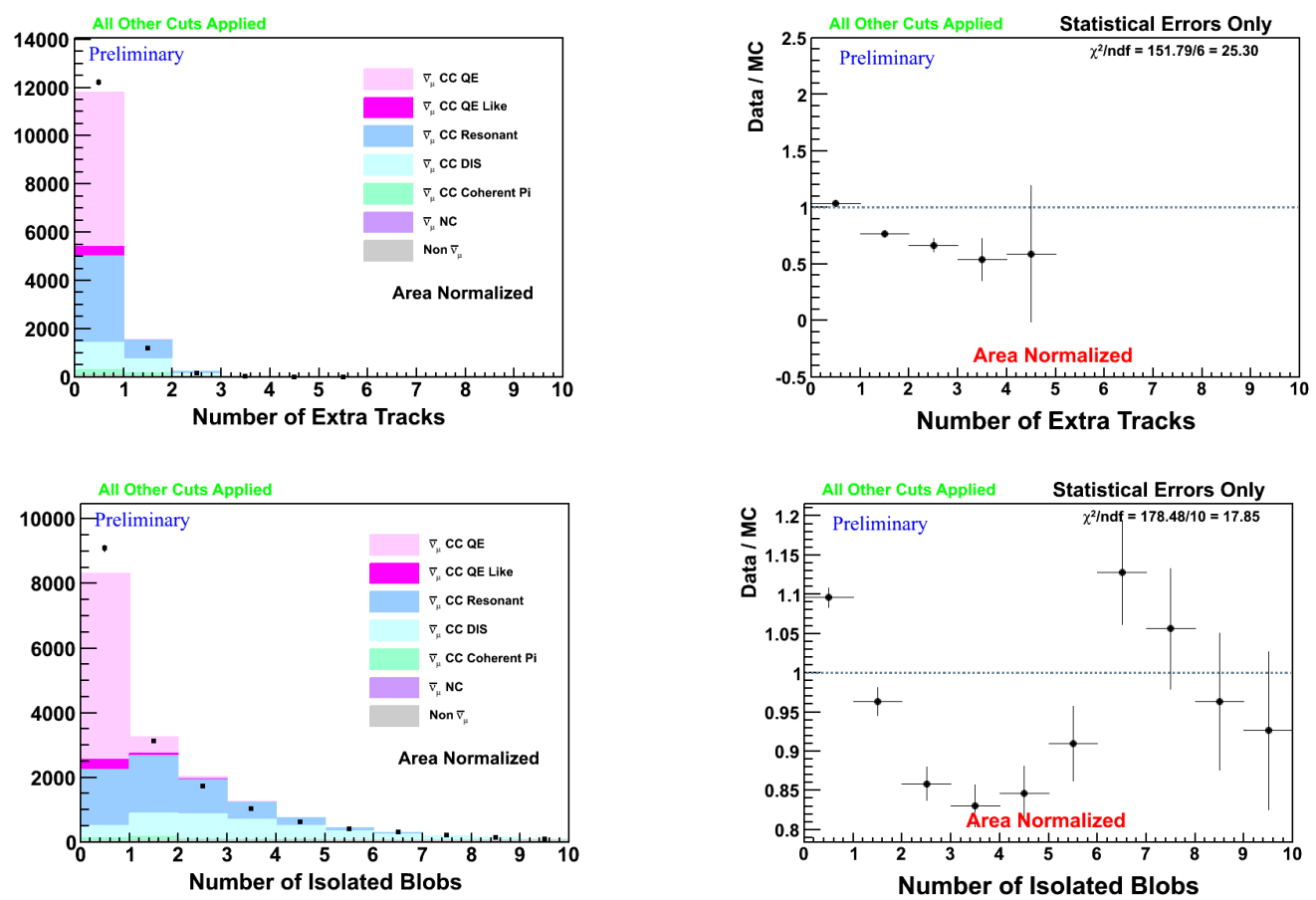

Figure 5.4: Data/MC overlays of the number tracks found in the event (excluding the muon track) and the number of isolated blobs found within the allowed time window. The $\chi^{2}$ value in data/MC ratios gives the consistency of the ratio with a flat line.

For activity found within that time window, we then make cuts on the allowed 
level of multiplicity. In this case, multiplicity refers to the number of additonal tracks and the number of isolated blobs (discussed in Section 4.4) within the previously described time window. In this analysis, we allow no extra tracks aside from the muon track. An additional track is likely to be from a proton or pion neither of which we generally expect in our signal sample. Additionally, we allow no more than a single isolated blob within the sample. For signal events in our sample, the isolated blob is presumed to be due to the neutron interacting within the detector. We expect no more than one such interaction. Using the simulation to predict what fraction of the signal we expect to have extra tracks or more than a single isolated blob, we find almost no signal to be present in the exclusions regions. These cuts generally improve the purity of our sample, but have a particularly large impact in improving the purity of the larger values of $\mathrm{Q}_{\mathrm{QE}}^{2}$ that we consider in this analysis. Distributions of each are visible in Figure 5.4.

Applying the two cuts on multiplicity rejects a substantial amount of background, but we find a sizeable number of background events still remain. To further reject this background, we cut on calometric energy summed up within the tracker and ECAL regions of the detector. We require that at least $1 \mathrm{MeV}$ be deposited in a cluster being considered in this sum. Within that cluster, no likely cross-talk hits may be present. We identify these hits in the same way we identified crosstalk clusters described in Section 4.3.3. If there are such hits, those hits within that cluster are vetoed, although the rest of the energy in that cluster may be considered. This requirement is to limit the amount of energy from cross-talk that enters our calorimetric sum. We also correct the visible energy found in qualifying clusters based upon known calorimetric variation within the detector. The resulting sum of calorimetric energy we refer to as recoil energy. 

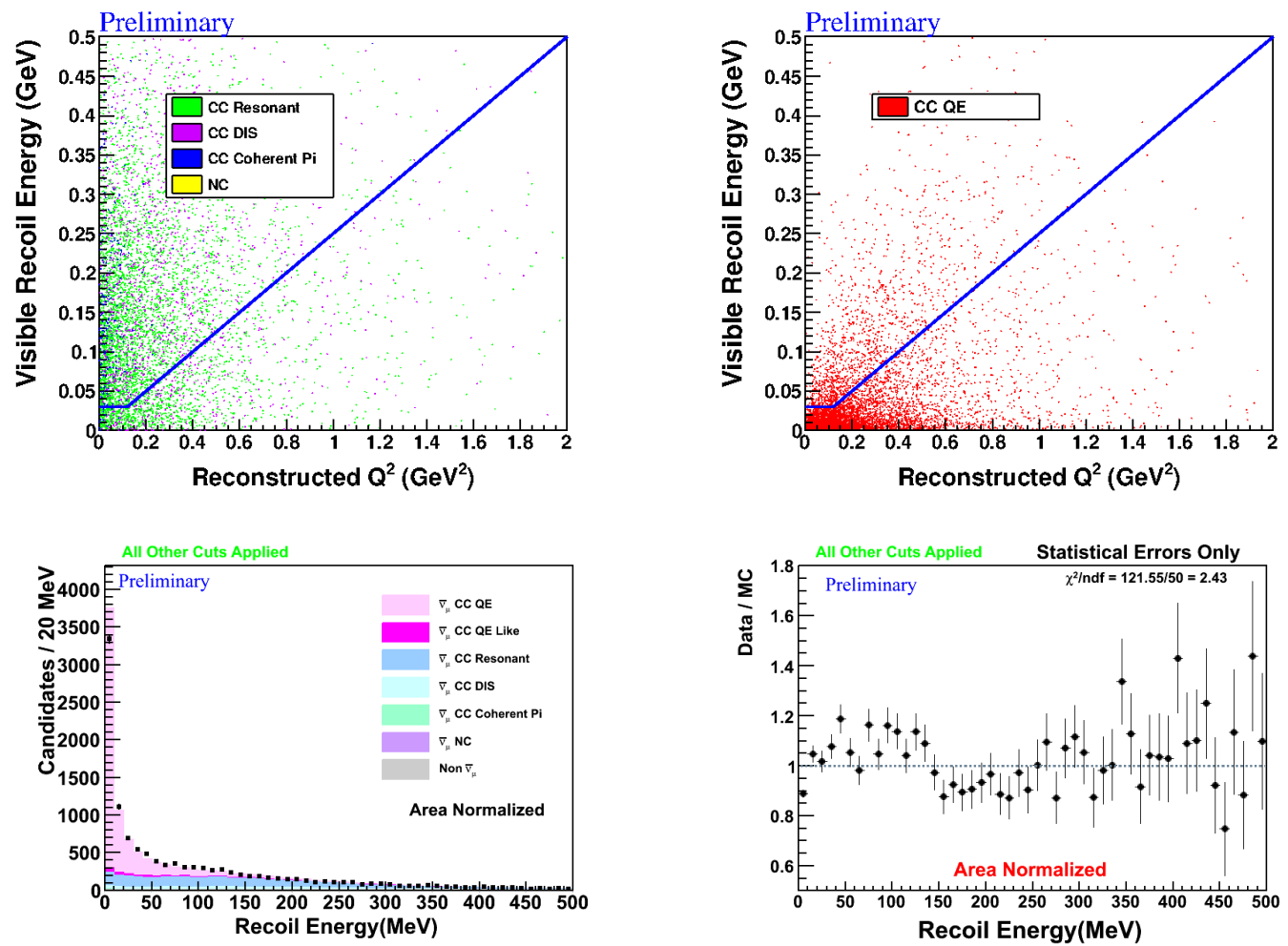

Figure 5.5: Recoil energy versus $\mathrm{Q}_{\mathrm{QE}}^{2}$ for signal and background. A data/MC overlay and data/MC ratio with a $\chi^{2}$ value showing the consistency of the ratio with a flat line are also shown.

We make a cut on recoil energy that is dependent upon the $\mathrm{Q}_{\mathrm{QE}}^{2}$ of an event. This cut is illustrated in Figure 5.5. The cut is $0.03 \mathrm{GeV}$ for $Q_{\mathrm{QE}}^{2}<0.12 \mathrm{GeV}^{2}$. Above this energy, we apply a cut with the form $\frac{\mathrm{Q}_{\mathrm{QE}}^{2}}{4}$. The diagonal line in Figure 5.5 shows where we make this cut. We use the $\mathrm{Q}_{\mathrm{QE}}^{2}$ cut since events with higher $\mathrm{Q}_{\mathrm{QE}}^{2}$ have larger amounts of energy transfered to the neutron. We expect higher energy neutrons to deposit more energy in the detector. Having a $\mathrm{Q}_{\mathrm{QE}}^{2}$ dependent cut improves the efficiency of the selection at higher values of $\mathrm{Q}_{\mathrm{QE}}^{2}$.

We suspect several issues may be causing the discrepancy between data and the simulation in the recoil energy plot in Figure 5.5. Based on studies discussed in 
Section 5.3 on systematic errors, we know there are sizable errors on the modeling of neutrons. We also do not correctly model cross-talk in the simulaiton. Other effects could also be present. An unidentified problem could be present in the muon energy reconstruction. There could also be issues with how the physics of the interaction is being modeled. We do a thorough examination of the sources of errors to ensure that discrepancies like those visible in the ratio plot in Figure 5.5 are fully accounted for in the systematic errors that we apply to the final result. After applying all cuts and vetoes, we find the distributions found in Figure 5.6. Additional plots for this analysis showing primarily reconstructed distributions that pass CCQE selection cuts may be found in Appendix A. Examples of two events that pass the CCQE selection criteria are shown in Figures 5.7 and 5.8.
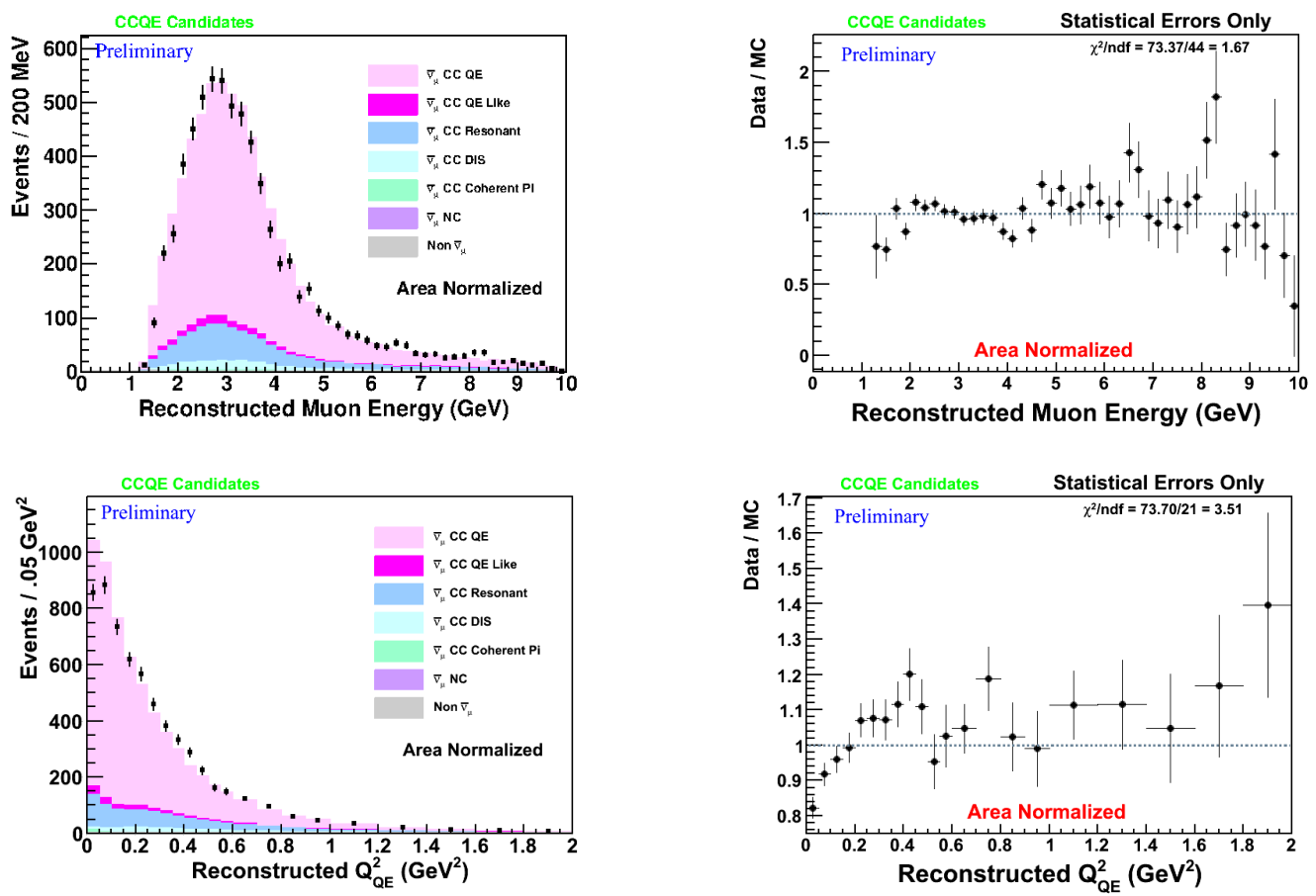

Figure 5.6: The muon energy and $\mathrm{Q}_{\mathrm{QE}}^{2}$ data/MC overlays for CCQE events. Data/MC ratios each with a $\chi^{2}$ value showing the consistency of the ratio with a flat line are also shown. 

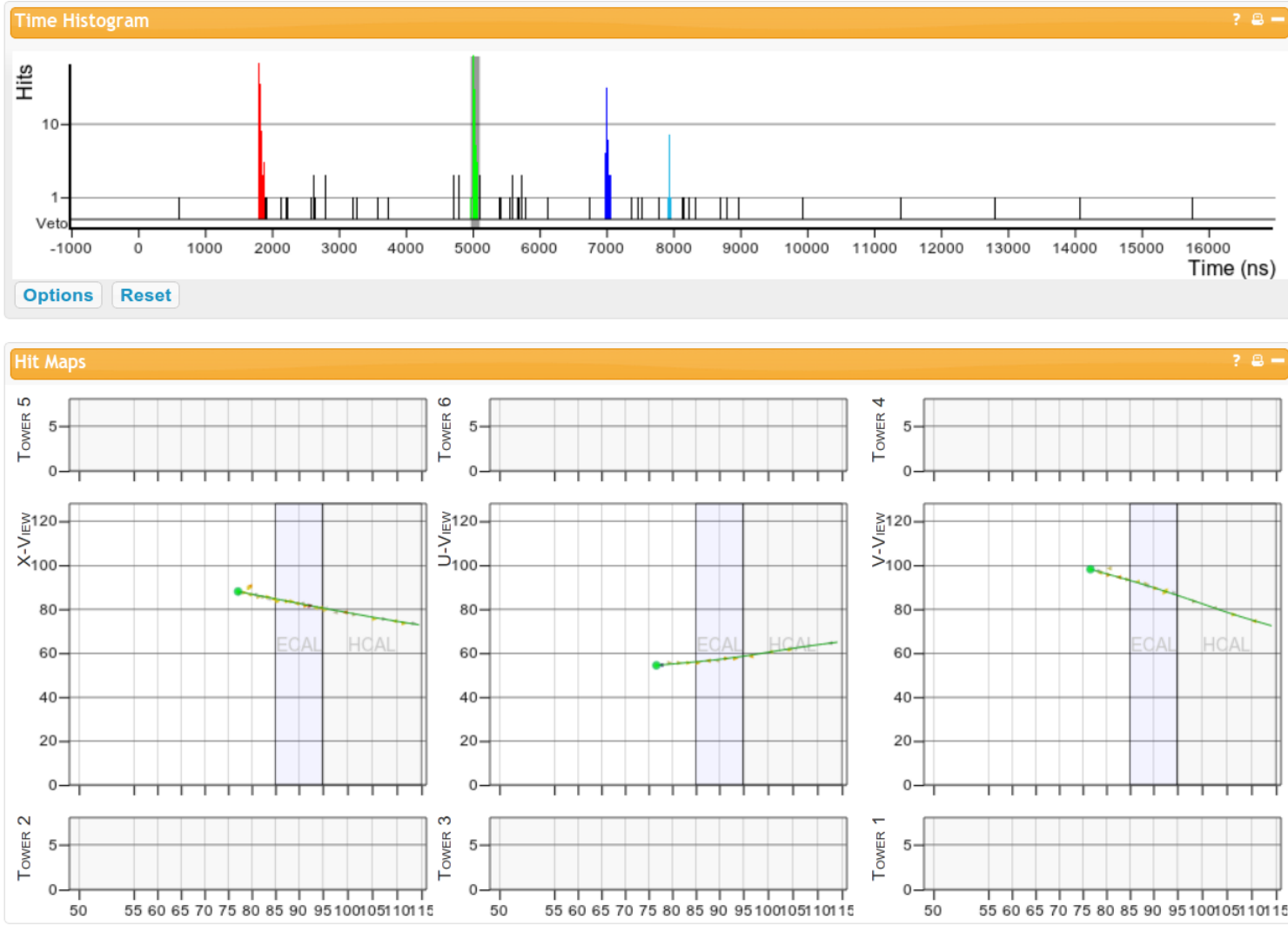

Figure 5.7: An event display of a CCQE interaction candiate in data. The time histogram shows the distribution of hits in time. The particular time slice being shown is highlighted in grey. The three different views are also visible. The reconstructed track is shown as a green line. The path of the muon through the three views is visible. In this event, little activity not associated with the muon track if found. Note that activity below roughtly $0.5 \mathrm{MeV}$ is not shown.

We find the purity and the product of acceptance and efficiency for the CCQE selection cuts outlined above. For these calculations, we determine the true number of events in the fiducial volune by criteria outlined in Section 5.1.4. Here, purity for a particular bin $\mathrm{i}$ is given by

$$
\mathrm{P}_{\mathrm{i}}=\frac{\mathrm{N}_{\text {Rec }, \mathrm{i}}^{\text {True }}}{\mathrm{N}_{\text {Rec,i }}},
$$

where $\mathrm{P}_{\mathrm{i}}$ is the purity for the $\mathrm{i}^{\text {th }}$ bin of a particular quantity, $\mathrm{N}_{\mathrm{Rec}, \mathrm{i}}$ is the number of events reconstructed in a particular bin, and $\mathrm{N}_{\text {Rec, }, \mathrm{i}}^{\text {True }}$ is the number of true CCQE 


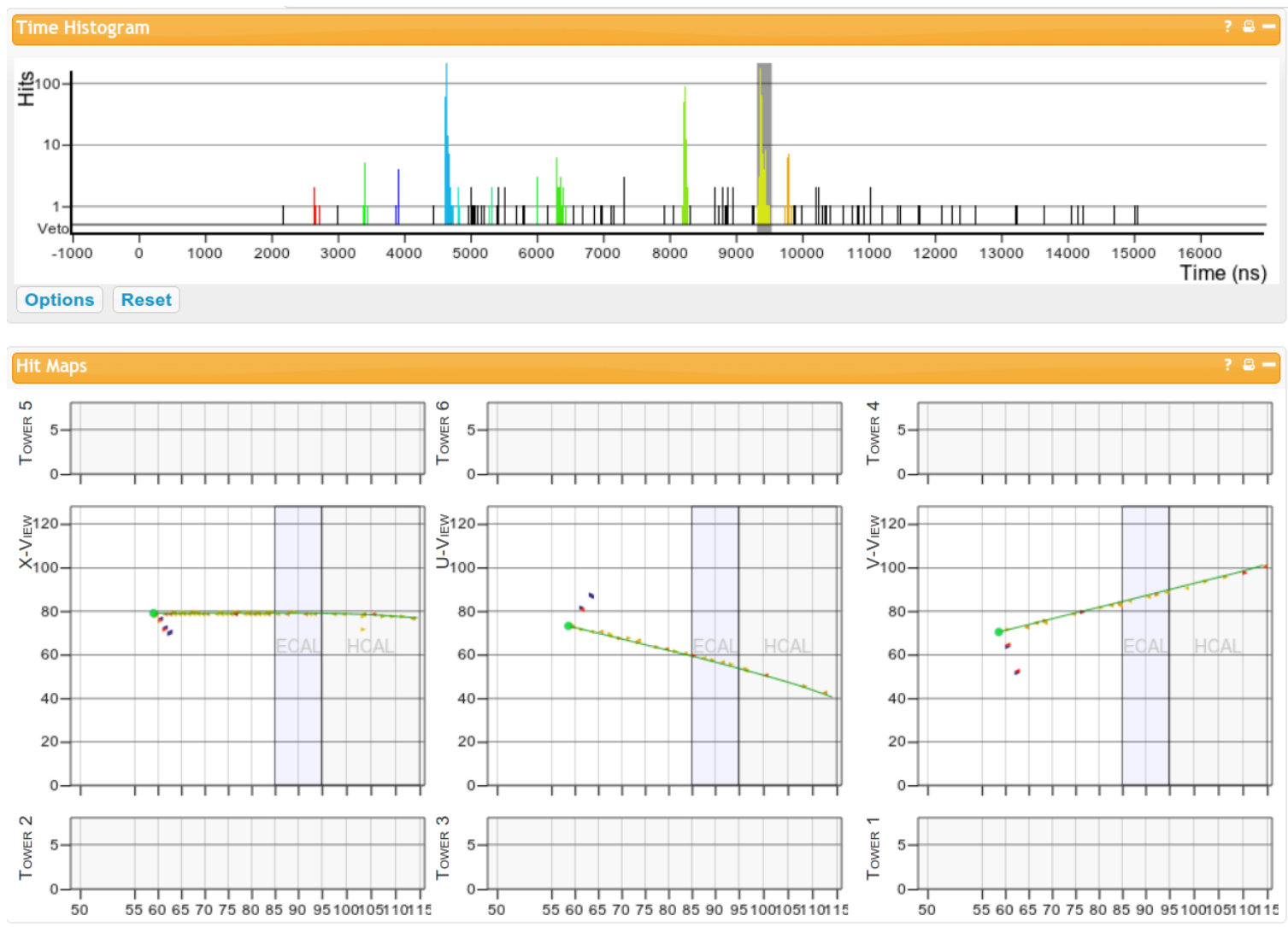

Figure 5.8: An event display of a CCQE interaction candiate in data. The time histogram shows the distribution of hits in time. The particular time slice being shown is highlighted in grey. The three different views are also visible. The reconstructed track is shown as a green line. The path of the muon through the three views is visible. Activity near the vertex is also visible. These hits become part of the vertex blob in this case. Note that activity below roughtly $0.5 \mathrm{MeV}$ is not shown.

events reconstructed in a particular bin. We find the purity as a function of muon energy, muon angle, neutrino energy, and $\mathrm{Q}_{\mathrm{QE}}^{2}$.

The product of acceptance and efficiency is given by

$$
\epsilon_{\mathrm{i}}=\epsilon_{\text {flat }} \frac{\mathrm{N}_{\mathrm{Gen}, \mathrm{i}}^{\mathrm{Rec}}}{\mathrm{N}_{\mathrm{Gen}, \mathrm{i}}}
$$

where the value $\epsilon_{\mathrm{i}}$ is the product of acceptance and efficiency for the $\mathrm{i}^{\text {th }}$ bin of a 
quanity, $\mathrm{N}_{\mathrm{Gen}, \mathrm{i}}$ is the number of true CCQE events generated within the fiducial volume, $\mathrm{N}_{\mathrm{Gen}, \mathrm{i}}^{\mathrm{Rec}}$ is the number of true CCQE events generated within the fiducial volume that are reconstructed, and $\epsilon_{\text {flat }}$ is a flat efficiency correction. We find the product of acceptance and efficiency for muon energy, muon angle, neutrino energy, and $\mathrm{Q}_{\mathrm{QE}}^{2}$. Further discussion of the product of acceptance and efficiency including the flat efficiency correction is found in Section 5.2.3.

Applying all selection cuts, we find a sample with approximately $80 \%$ purity. Our purity is relatively flat across muon energy with a slight fall off of the observed purity at increasing values of muon angle, neutrino energy, and $\mathrm{Q}_{\mathrm{QE}}^{2}$. The resulting purity and the product of acceptance and efficiency are shown in Figure 5.9.

\subsubsection{Defining the Signal Sample in Monte Carlo}

A definition of CCQE scattering needs to be established since there can be ambiguity at the generator level over what constitutes signal. It is pertinent to know what should constitute signal and background when correcting for acceptances and efficiencies or subtracting background. In this analysis, we define CCQE interactions in the simulation based on the generator level definition in GENIE with the exclusion of any type of interaction that has a charm final state. We apply all other cuts that we apply to data to select signal events.

We also consider the case where we have feed down from higher energy CCQE events or leakage into our fiducial due to having a finite vertex resolution. Consequently, we consider an event to be signal if it had less than $12 \mathrm{GeV}$ at the generator level and if it had an interaction vertex at the generator level within $1 \mathrm{~cm}$ of the fiducial volume in the transverse direction and one module in the longitudinal direction. Note that in each case, the event must have $10 \mathrm{GeV}$ or less in reconstructed 

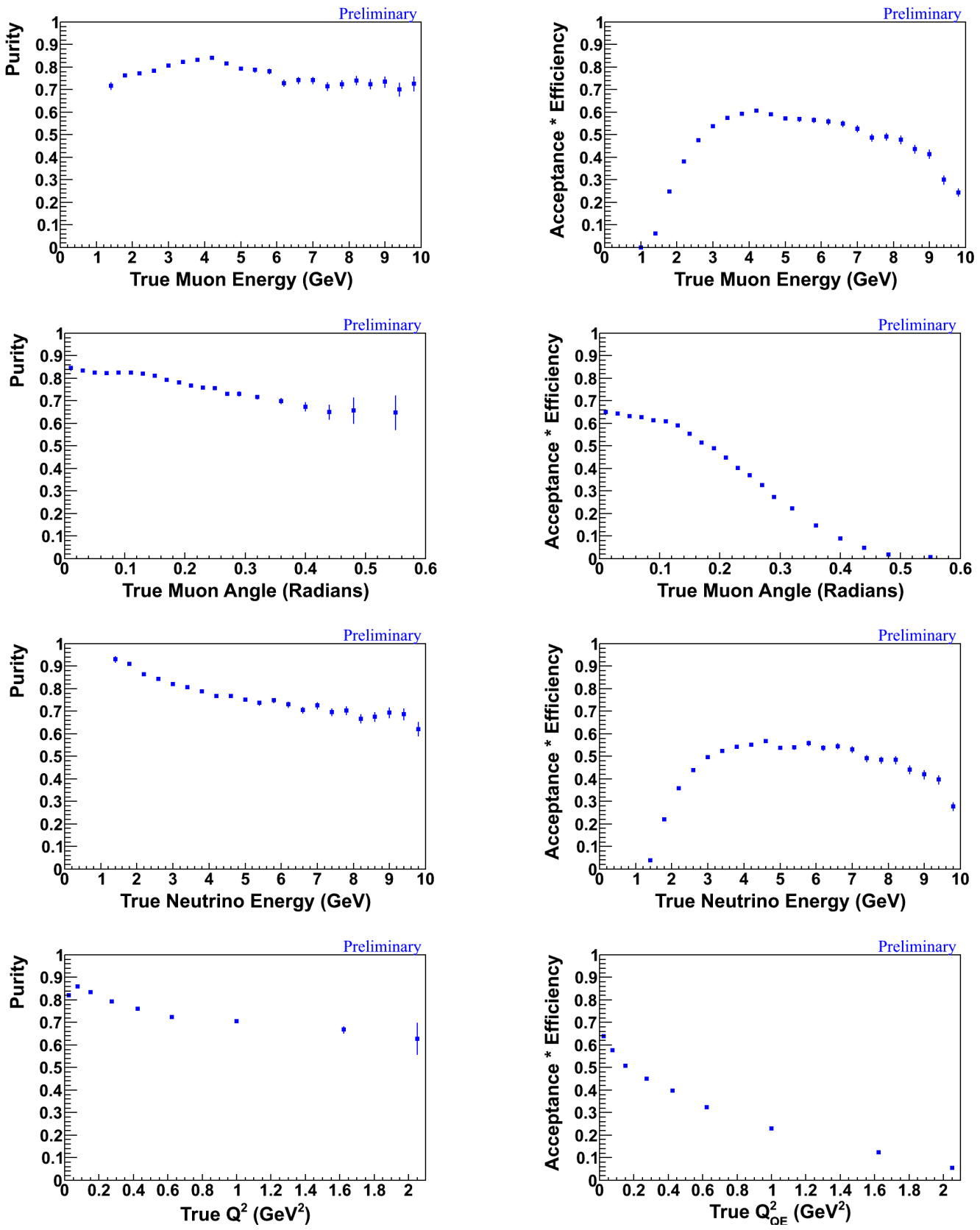

Figure 5.9: The purity and product of acceptance and efficiency of our sample across muon energy, muon angle, neutrino energy, and $\mathrm{Q}_{\mathrm{QE}}^{2}$. All flat efficiency effects are also included in these distributions. 
energy and be reconstructed within the fiducial volume. This is done to miminize the number of CCQE events we consider background. Some CCQE events will leak into the sample and others will leak out. An improperly composed definition would lead to signal events being subtracted out as background and an under-reporting of the cross-section. In total, we have less than $0.25 \%$ of CCQE events identified as background. These are mostly events with greater than $12 \mathrm{GeV}$ of energy at the generator level although some events do not fall within the looser volume allowed for event generation. These events are subtracted from the sample in the manner described in Section 5.2.1.

\subsection{Calculation of the Differential CCQE Cross- Section $\frac{\mathrm{d} \sigma}{\mathrm{d} \mathrm{Q}_{\mathrm{QE}}^{2}}$}

To extract $\frac{\mathrm{d} \sigma}{\mathrm{dQ}_{\mathrm{QE}}^{2}}$, we must account for backgrounds, acceptances, efficiencies, and detector smearing. The resulting distribution must then be properly normalized. Together, this procedure is given by

$$
\left(\frac{\mathrm{d} \sigma}{\mathrm{d} Q_{\mathrm{QE}}^{2}}\right)_{\mathrm{i}}=\frac{\sum_{\mathrm{j}}\left(\mathrm{M}_{\mathrm{ij}}^{-1}\left(\mathrm{~N}_{\mathrm{data}, \mathrm{j}}-\mathrm{N}_{\mathrm{data}, \mathrm{j}}^{\mathrm{bkgd}}\right)\right)}{\epsilon_{\mathrm{i}} \Phi \mathrm{T} \Delta \mathrm{Q}_{\mathrm{QE}, \mathrm{i}}^{2}},
$$

where $\left(\frac{\mathrm{d} \sigma}{\mathrm{d} \mathrm{Q}_{\mathrm{QE}}^{2}}\right)_{\mathrm{i}}$ is the differential cross-section averaged over bin $\mathrm{i}$, the term $\mathrm{M}_{\mathrm{ij}}^{-1}$ is the unfolding matrix element, the value $\mathrm{N}_{\mathrm{data}, \mathrm{j}}$ is the number of entries reconstructed in $Q_{Q E}^{2}$ bin $j$, the value $N_{\text {data,j }}^{\text {bkgd }}$ is the number of estimated background entries in bin $\mathrm{j}$ of reconstructed $\mathrm{Q}_{\mathrm{QE}}^{2}, \epsilon_{\mathrm{i}}$ is the efficiency in selecting the $\mathrm{i}^{\text {th }}$ entry of the unfolded $\mathrm{Q}_{\mathrm{QE}}^{2}$ distribution, $\Phi$ is the integrated flux, $\mathrm{T}$ is the number of protons within the 
fiducial volume, and $\Delta \mathrm{Q}_{\mathrm{QE}, \mathrm{i}}^{2}$ is the bin width of the $\mathrm{i}^{\text {th }} \mathrm{Q}_{\mathrm{QE}}^{2}$ bin. We use binning where the edges of each bin in units of $\mathrm{GeV}^{2}$ are given by: $0,0.05,0.1,0.2,0.35$, $0.5,0.75,1.25,2.0$. We consider events with $\mathrm{Q}_{\mathrm{QE}}^{2}>2.0 \mathrm{GeV}^{2}$ in the calculation, but only report a differential cross-section below $2.0 \mathrm{GeV}^{2}$. The differential cross-section with all terms applied can be found in Figure 5.24. A description of how each of these parameters is extracted follows below.

\subsubsection{Backgrounds}

Several types of backgrounds enter the signal sample: non-CCQE anti-neutrino interactions, neutrino interactions with a misreconstructed charge, and rock muons originating from outside of the detector. We estimate the rock muon background by doing a scan of events that pass our signal selection cuts. Although most rock muons are vetoed by either the fiducial vertex requirements, the deadtime cut, or the cut on upstream activity, a small number still enters the sample. To identify these, we perform a scan using the Arachne event display [104]. Out of 653 signal events that were scanned, 4 were found to be rock muons. Based on our total number of events in our sample, we therefore estimate $0.6 \% \pm 0.3 \%$ of our data sample is rock muons.

To estimate the shape of $\mathrm{Q}_{\mathrm{QE}}^{2}$ and recoil energy distributions for the rock muon background, we first select rock muon tracks in data that enter the detector. We consider only those events that are matched to a positive muon in MINOS. We further require tracks that pass the previous requirements to pass through the first plane of our fiducial volume within a hexagon with an apothem of $850 \mathrm{~mm}$. We reconstruct energy and $\mathrm{Q}_{\mathrm{QE}}^{2}$ assuming CCQE kinematics. We apply the same selection on extra tracks, number of allowed isolated blobs, and recoil energy that we use to select signal events. We then normalize these distributions to our signal sample 

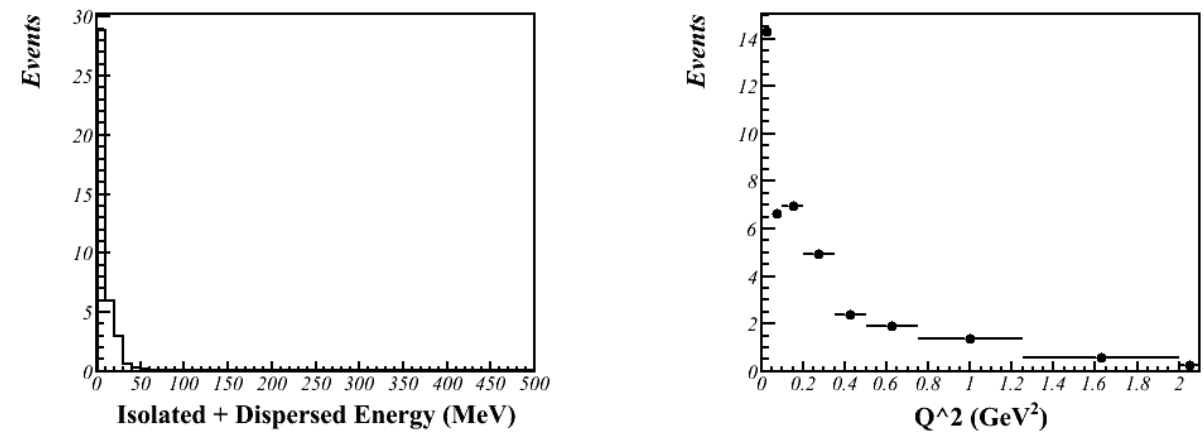

Figure 5.10: The estimated $\mathrm{Q}_{\mathrm{QE}}^{2}$ and recoil energy distributions for the rock muon background in the sample normalized to the data sample. Note that the $Q^{2}$ found in the plot is reconstructed using CCQE kinematics.

based on the $0.6 \%$ estimate for the rock muon background. The resulting distributions of $\mathrm{Q}_{\mathrm{QE}}^{2}$ and recoil energy for the rock muon background are shown in Figure 5.10. This background is subtracted off of the $\mathrm{Q}_{\mathrm{QE}}^{2}$ and recoil energy distributions.

After subtracting rock muons from the sample, we then consider other backgrounds. The background from non-CCQE anti-neutrino interactions and misreconstructed neutrino interactions are initially estimated using the simulation. Issues exist with using the siulation for this purpose since uncertainties on background processes can be large and even then not well modeled. Particular concern exists over the Rein-Seghal model used to predict single pion production in neutrino and anti-neutrino interactions [96]. To constrain background predictions, we perform a template fit of background distributions in the simulation to data.

The template fit is done using recoil energy distributions for four separate bins of $\mathrm{Q}_{\mathrm{QE}}^{2}$. We perform the fit for the following combined bins of $\mathrm{Q}_{\mathrm{QE}}^{2}, \mathrm{Q}_{\mathrm{QE}}^{2}<0.1 \mathrm{GeV}^{2}$, $0.1 \leq \mathrm{Q}_{\mathrm{QE}}^{2}<0.35 \mathrm{GeV}^{2}, 0.35 \leq \mathrm{Q}_{\mathrm{QE}}^{2}<0.75 \mathrm{GeV}^{2}$, and $\mathrm{Q}_{\mathrm{QE}}^{2} \geq 0.75 \mathrm{GeV}^{2}$. We use the TFractionFitter package in ROOT which is based on a method by R. J. Barlow and C. Beeston $[94,107]$. We make shape templates for each of the four bins of $\mathrm{Q}_{\mathrm{QE}}^{2}$ 
for true signal and background events in Monte Carlo. The TFractionFitter then varies the normalization of these templates to match the correpsonding data recoil distribution for the same bin of $\mathrm{Q}_{\mathrm{QE}}^{2}$. The fit allows the normalization within each bin of the recoil energy distribution being fit to vary within statistical errors. The fit returns the fraction of events that are deemed to be background. We then take the ratio of the level of background returned by the fit over the level of background predicted by Monte Carlo to scale the background in each of the $\mathrm{Q}_{\mathrm{QE}}^{2}$ bins, so that

$$
\mathrm{N}_{\text {data, } \mathrm{i}}^{\text {bkgd }}=\mathrm{f}_{\text {fit, }, \mathrm{i}} \frac{\mathrm{N}_{\mathrm{MC}, \mathrm{i}}^{\mathrm{bkgd}}}{\mathrm{N}_{\mathrm{MC}, \mathrm{i}}} \mathrm{N}_{\text {data }, \mathrm{i}}
$$

where $\mathrm{i}$ is a particular bin of $\mathrm{Q}_{\mathrm{QE}}^{2}, \mathrm{f}_{\mathrm{fit}, \mathrm{i}}$ is the result of the template fit in bin $\mathrm{i}, \mathrm{N}_{\mathrm{MC}, \mathrm{i}}$ is the total number of $\mathrm{MC}$ events in bin $\mathrm{i}, \mathrm{N}_{\mathrm{MC}, \mathrm{i}}^{\mathrm{bkgd}}$ is the background predicted by Monte Carlo to be in bin $\mathrm{i}$, and $\mathrm{N}_{\text {data, } \mathrm{i}}^{\mathrm{bkgd}}$ is the predicted level of background in data for bin i. The recoil distributions prior to the fit are shown in Figure 5.11. The recoil distributions after the fit along with the extracted value from the fit are shown in Figure 5.12. The fit results for each bin of $\mathrm{Q}_{\mathrm{QE}}^{2}$ is given in Figure 5.13. This background was then subtracted from the relevant bin of our $\mathrm{Q}_{\mathrm{QE}}^{2}$ distribution. See Figures 5.14 and 5.15 for plots of the $\mathrm{Q}_{\mathrm{QE}}^{2}$ distribution prior to and after background subtraction, respectively.

We also considered what systematic errors result from the previously described methodology of background subtraction. For the rock muon background, see Section 5.3.1 for how a systematic error was extracted. For the wrong sign neutrino background and non-CCQE background, we include both uncertainty from the template fit and statistical errors on the Monte Carlo distribution prior to the fit. These errors are both included during background subtraction and propagate to the $\frac{\mathrm{d} \sigma}{\mathrm{dQ}_{\mathrm{QE}}^{2}}$ 
statistical uncertainty.
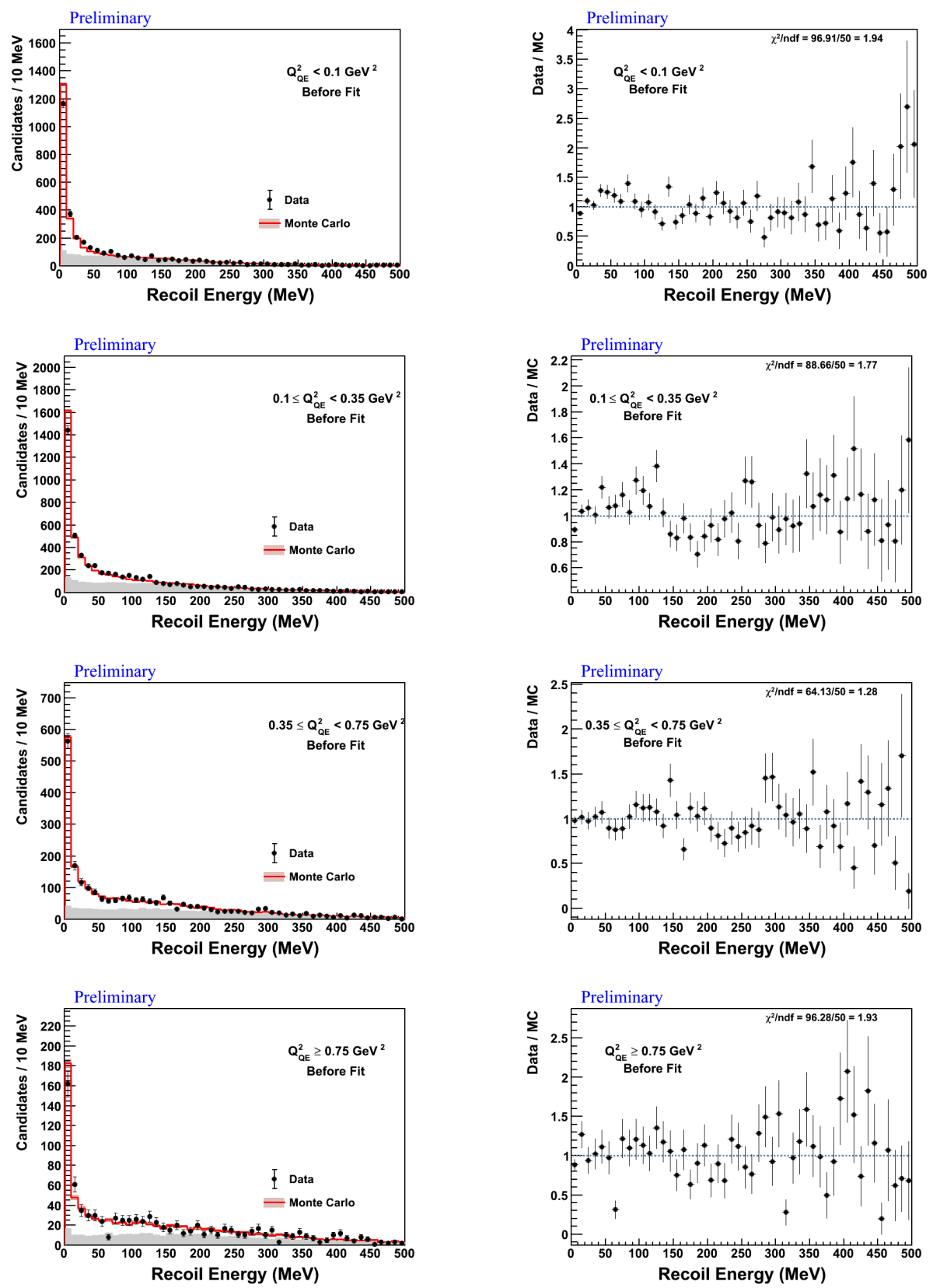

Figure 5.11: Area normalized data/MC overlays and ratios for four separate $\mathrm{Q}_{\mathrm{QE}}^{2}$ bins of recoil energy before the template fit. The $\chi^{2}$ values are the result of a comparison of a ratio to a flat line. 

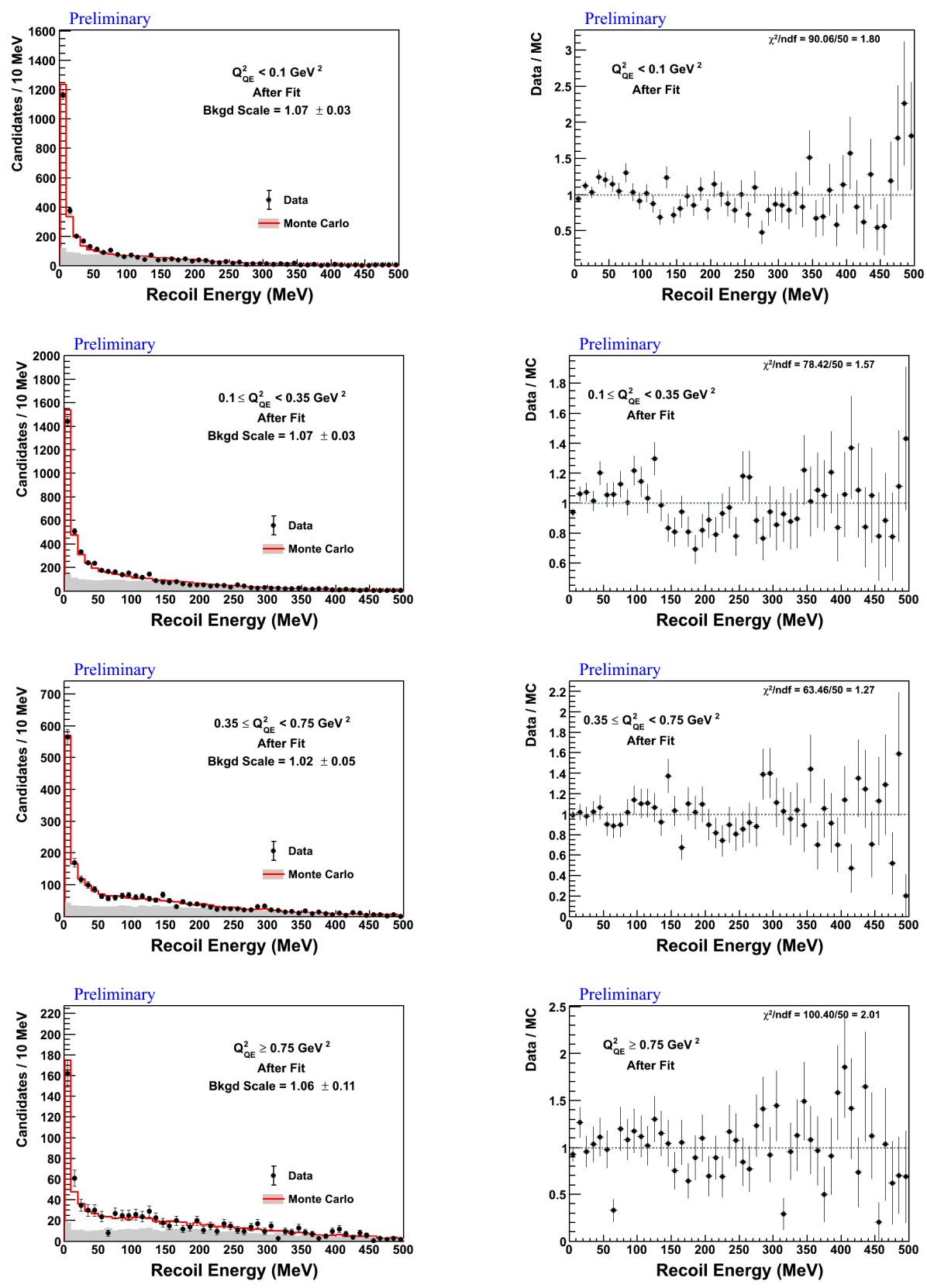

Figure 5.12: Area normalized data/MC overlays and ratios for four separate $\mathrm{Q}_{\mathrm{QE}}^{2}$ bins of recoil energy after the template fit. The $\chi^{2}$ values are the result of a comparison of a ratio to a flat line. 


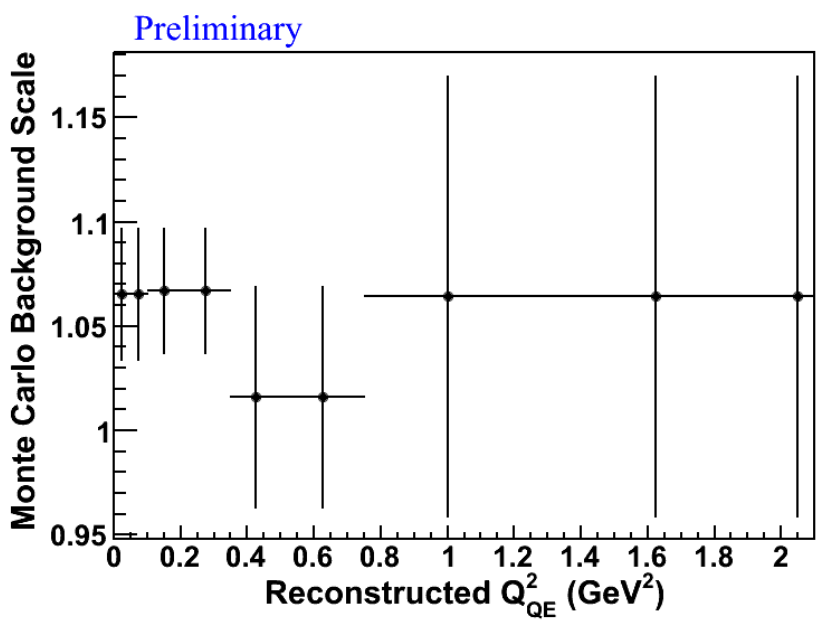

Figure 5.13: The fit results as a function of $\mathrm{Q}_{\mathrm{QE}}^{2}$ using the same binning as the $\frac{\mathrm{d} \sigma}{\mathrm{dQ}_{\mathrm{QE}}^{2}}$ measurement. The fit is performed for groups of bins.
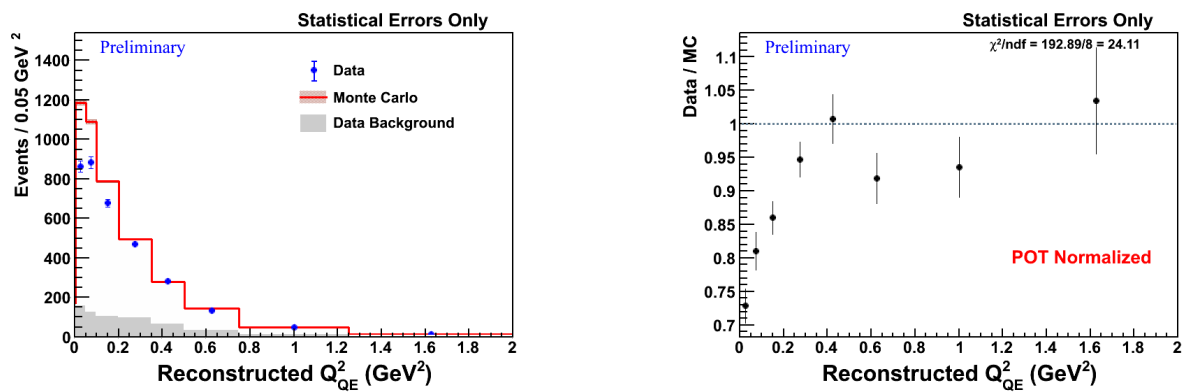

Figure 5.14: Data/MC overlay and ratio before background subtraction is applied. Backgrounds are shown in light grey. The $\chi^{2}$ values are the result of a comparison of the ratio to a flat line.

\subsubsection{Unfolding Detector Smearing}

After backgrounds have been subtracted, we unfold the reconstructed $\mathrm{Q}_{\mathrm{QE}}^{2}$ distribution to account for detector smearing effects. We use this procedure since we have a finite resolution within the detector in both muon energy and angle, which are the observables in this analysis (see resolution Tables A.2 and A.3 in Appendix A). Overall, we see a $\mathrm{Q}_{\mathrm{QE}}^{2}$ resolution between $25 \%$ and $30 \%$ as seen in Figure A.25 in 

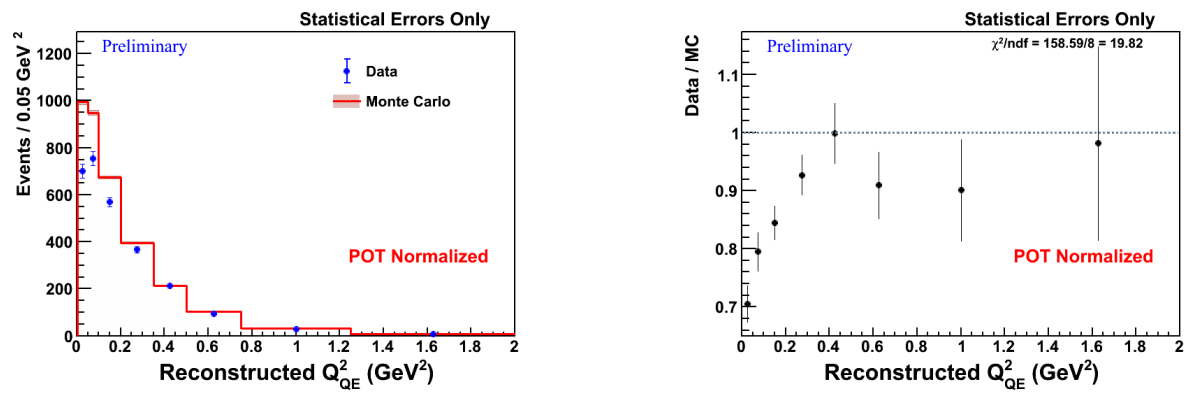

Figure 5.15: Data/MC overlay and ratio after background subtraction is applied. The $\chi^{2}$ values are the result of a comparison of the ratio to a flat line. Data has a significant deficit of events at low $\mathrm{Q}_{\mathrm{QE}}^{2}$ relative to the simulation.

Appendix A. The unsmearing procedure corrects for these resolution effects as well as reconstruction biases. One such bias we rely on the unfolding to correct is the energy lost by a muon in the ArgoNeuT detector. We employ a matrix inversion method to accomplish this.

To construct this matrix, we first create a smearing matrix with the same binning as the $\frac{\mathrm{d} \sigma}{\mathrm{dQ}_{\mathrm{QE}}^{2}}$ distribution along with an additional column and row for overflow entries. Any underflow entries, which can happen in certain cases in the calculation of $\mathrm{Q}_{\mathrm{QE}}^{2}$, are added to the first appropriate bin in the matrix. That matrix maps true $\mathrm{Q}_{\mathrm{QE}}^{2}$ values calculated using the true muon kinematics to reconstructed $\mathrm{Q}_{\mathrm{QE}}^{2}$ found using reconstructed muon kinematics. This initial matrix is filled with true CCQE events which pass the signal selection cuts for this analysis. Each entry is then normalized by the total number of true entries for a particular bin of reconstructed $\mathrm{Q}_{\mathrm{QE}}^{2} \cdot$

We do not use the GENIE generator level value of $\mathrm{Q}_{\mathrm{QE}}^{2}$ in the simulation to avoid being sensitive to the RFG Model and other nuclear models used in the simulation. Concerns exist about the accuracy of these and we wish to avoid introducing systematic error by allowing the unfolding procedure to be sensitive to such ef- 
fects. Moreover, it is a goal of MINER $\nu \mathrm{A}$ to study how well these models describe MINER $\nu \mathrm{A}$ data.

The smearing matrix is given by

$$
\mathrm{M}_{\mathrm{ij}}=\frac{\mathrm{N}_{\mathrm{true}, \mathrm{j}}^{\mathrm{rec}, \mathrm{i}}}{\mathrm{N}_{\text {true }, \mathrm{j}}^{\text {rec }}}
$$

where $\mathrm{M}_{\mathrm{ij}}$ is the smearing matrx, the term $\mathrm{N}_{\text {true,j }}^{\mathrm{rec}}$ is the number of signal candidates in Monte Carlo that are generated in bin $\mathrm{j}$, and the term $\mathrm{N}_{\text {true, } \mathrm{j}}^{\mathrm{rec}, \mathrm{i}}$ is the subset of those candidates that are also reconstructed in bin i. We then invert this matrix such that,

$$
\mathrm{N}_{\text {data,i }}^{\text {true }}=\sum_{j} \mathrm{~N}_{\text {data,j }}^{\text {rec }} \mathrm{M}_{\mathrm{ij}}^{-1}
$$

Prior to inversion, we calculate binomial errors for the smearing matrix. Due to the finite statistics of the sample used to make the smearing matrix, some error exists on the unsmearing matrix. Errors from the smearing matrix and errors from the reconstructed distribution are propagated to the unsmeared distribution using a method described by M. Lefebvre, R.K. Keeler, R. Sobie, and J. White [108].

The smearing and unfolding matrices along with the errors for each entry are shown in Tables 5.1, 5.2, 5.3, and 5.4. We select the size of the bin width to maximize the effectiveness of this unfolding method. The matrices are constructed using Monte Carlo samples approximately 10 times the size of the data sample. The unfolded $\mathrm{Q}_{\mathrm{QE}}^{2}$ distribution is shown in Figure 5.16. Note that we do not show entries in the overflow bin as statistics are poor and systematic errors are very large in the region where $\mathrm{Q}_{\mathrm{QE}}^{2}>2 \mathrm{GeV}^{2}$. 

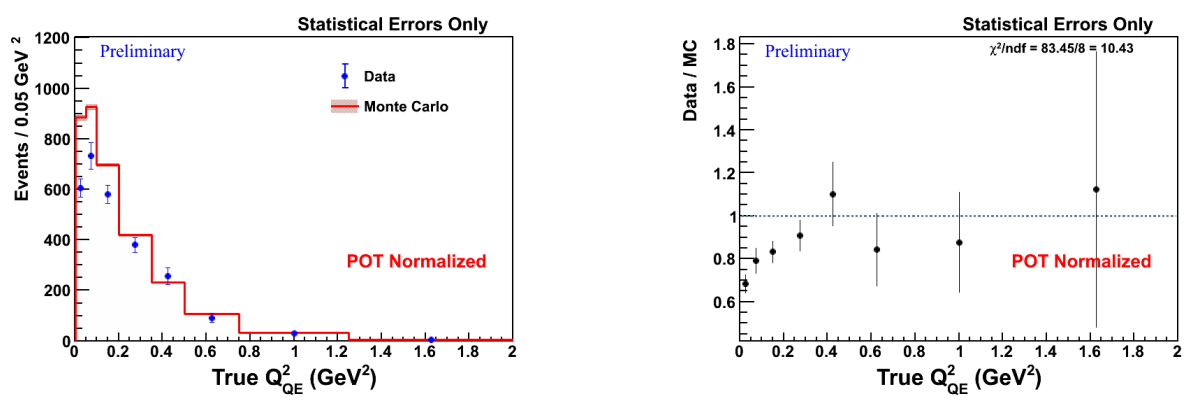

Figure 5.16: Data/MC overlays and ratios for unfolded $\mathrm{Q}_{\mathrm{QE}}^{2}$. The $\chi^{2}$ values are the result of a comparison of the ratio to a flat line.

\begin{tabular}{|c|c|c|c|c|c|c|c|c|c|}
\hline$Q_{R e}^{2}$ & $\begin{array}{c}Q_{\text {True }}^{2} \\
{[0,0.05)} \\
\end{array}$ & {$[0.05,0.1)$} & {$[0.1,0.2)$} & {$[0.2,0.35)$} & {$[0.35,0.5)$} & {$[0.5,0.75)$} & {$[0.75,1.25)$} & {$[1.25,2.0)$} & {$[2.0, \infty)$} \\
\hline$[-\infty, 0.05)$ & 0.916 & 0.077 & 0.005 & 0.001 & 0.000 & 0.000 & 0.000 & 0.000 & 0.000 \\
\hline$[0.05,0.1)$ & 0.187 & 0.682 & 0.120 & 0.008 & 0.002 & 0.000 & 0.001 & 0.000 & 0.000 \\
\hline$[0.1,0.2)$ & 0.006 & 0.174 & 0.690 & 0.113 & 0.009 & 0.004 & 0.002 & 0.001 & 0.000 \\
\hline$[0.2,0.35)$ & 0.001 & 0.004 & 0.203 & 0.641 & 0.112 & 0.027 & 0.008 & 0.003 & 0.002 \\
\hline$[0.35,0.5)$ & 0.001 & 0.000 & 0.017 & 0.274 & 0.496 & 0.163 & 0.036 & 0.007 & 0.005 \\
\hline$[0.5,0.75)$ & 0.001 & 0.000 & 0.002 & 0.041 & 0.230 & 0.531 & 0.159 & 0.024 & 0.012 \\
\hline$[0.75,1.25)$ & 0.001 & 0.000 & 0.001 & 0.004 & 0.028 & 0.232 & 0.578 & 0.122 & 0.034 \\
\hline$[1.25,2.0)$ & 0.007 & 0.000 & 0.000 & 0.003 & 0.004 & 0.018 & 0.283 & 0.529 & 0.157 \\
\hline$[2.0, \infty)$ & 0.013 & 0.000 & 0.000 & 0.004 & 0.008 & 0.015 & 0.047 & 0.186 & 0.728 \\
\hline
\end{tabular}

Table 5.1: The smearing matrix that relates true $\mathrm{Q}_{\mathrm{QE}}^{2}$ to reconstructed $\mathrm{Q}_{\mathrm{QE}}^{2}$. This matrix is inverted to find the unfolding matrix. The bins of $\mathrm{Q}_{\mathrm{QE}}^{2}$ are in units of $\mathrm{GeV}^{2}$.

\begin{tabular}{|c|c|c|c|c|c|c|c|c|c|}
\hline $\mathrm{Q}_{\text {Rec }}^{2}$ & $\begin{array}{c}\mathrm{Q}_{\text {True }}^{2} \\
{[0,0.05)}\end{array}$ & {$[0.05,0.1)$} & {$[0.1,0.2)$} & {$[0.2,0.35)$} & {$[0.35,0.5)$} & {$[0.5,0.75)$} & {$[0.75,1.25)$} & {$[1.25,2.0)$} & {$[2.0, \infty)$} \\
\hline$[-\infty, 0.05)$ & 0.003 & 0.003 & 0.001 & 0.000 & 0.000 & 0.000 & 0.000 & 0.000 & 0.000 \\
\hline$[0.05,0.1)$ & 0.004 & 0.005 & 0.003 & 0.001 & 0.000 & 0.000 & 0.000 & 0.000 & 0.000 \\
\hline$[0.1,0.2)$ & 0.001 & 0.003 & 0.004 & 0.003 & 0.001 & 0.001 & 0.000 & 0.000 & 0.000 \\
\hline$[0.2,0.35)$ & 0.000 & 0.001 & 0.004 & 0.004 & 0.003 & 0.001 & 0.001 & 0.000 & 0.000 \\
\hline$[0.35,0.5)$ & 0.000 & 0.000 & 0.002 & 0.005 & 0.006 & 0.004 & 0.002 & 0.001 & 0.001 \\
\hline$[0.5,0.75)$ & 0.000 & 0.000 & 0.001 & 0.003 & 0.006 & 0.007 & 0.005 & 0.002 & 0.001 \\
\hline$[0.75,1.25)$ & 0.001 & 0.000 & 0.000 & 0.001 & 0.003 & 0.007 & 0.009 & 0.006 & 0.003 \\
\hline$[1.25,2.0)$ & 0.003 & 0.000 & 0.000 & 0.002 & 0.002 & 0.005 & 0.016 & 0.018 & 0.013 \\
\hline$[2.0, \infty)$ & 0.010 & 0.000 & 0.000 & 0.006 & 0.007 & 0.010 & 0.018 & 0.034 & 0.039 \\
\hline
\end{tabular}

Table 5.2: Uncertainties on the smearing matrix. The bins of $\mathrm{Q}_{\mathrm{QE}}^{2}$ are in units of $\mathrm{GeV}^{2}$.

\begin{tabular}{|c|c|c|c|c|c|c|c|c|c|}
\hline $\mathrm{Q}_{\mathrm{Rec}}^{2}$ & $\begin{array}{c}\mathrm{Q}_{\text {True }}^{2} \\
{[0,0.05)}\end{array}$ & {$[0.05,0.1)$} & {$[0.1,0.2)$} & {$[0.2,0.35)$} & {$[0.35,0.5)$} & {$[0.5,0.75)$} & {$[0.75,1.25)$} & {$[1.25,2.0)$} & {$[2.0, \infty)$} \\
\hline$[-\infty, 0.05)$ & 1.118 & -0.131 & 0.016 & -0.003 & 0.001 & -0.001 & 0.000 & -0.000 & 0.000 \\
\hline$[0.05,0.1)$ & -0.319 & 1.575 & -0.282 & 0.034 & -0.010 & 0.003 & -0.002 & 0.001 & -0.000 \\
\hline$[0.1,0.2)$ & 0.075 & -0.417 & 1.607 & -0.296 & 0.043 & -0.010 & -0.001 & -0.000 & 0.000 \\
\hline$[0.2,0.35)$ & -0.024 & 0.132 & -0.553 & 1.834 & -0.423 & 0.044 & -0.006 & -0.004 & -0.001 \\
\hline$[0.35,0.5)$ & 0.010 & -0.066 & 0.279 & -1.119 & 2.618 & -0.777 & 0.068 & -0.009 & -0.005 \\
\hline$[0.5,0.75)$ & -0.004 & 0.021 & -0.093 & 0.377 & -1.213 & 2.517 & -0.646 & 0.053 & -0.013 \\
\hline$[0.75,1.25)$ & 0.001 & -0.006 & 0.028 & -0.121 & 0.405 & -1.078 & 2.236 & -0.477 & 0.012 \\
\hline$[1.25,2.0)$ & -0.010 & 0.004 & -0.013 & 0.055 & -0.200 & 0.528 & -1.228 & 2.311 & -0.448 \\
\hline$[2.0, \infty)$ & -0.018 & 0.001 & 0.003 & -0.013 & 0.025 & -0.109 & 0.182 & -0.560 & 1.488 \\
\hline
\end{tabular}

Table 5.3: The unfolding matrix used to transform reconstructed $\mathrm{Q}_{\mathrm{QE}}^{2}$ distributions to true $\mathrm{Q}_{\mathrm{QE}}^{2}$ distributions. This is the inverse of Table 5.1. The bins of $\mathrm{Q}_{\mathrm{QE}}^{2}$ are in units of $\mathrm{GeV}^{2}$. 


\begin{tabular}{|c|c|c|c|c|c|c|c|c|c|}
\hline $\mathrm{Q}_{\mathrm{Rec}}^{2}$ & $\begin{array}{c}\mathrm{Q}_{\text {True }}^{2} \\
{[0,0.05)} \\
\end{array}$ & {$[0.05,0.1)$} & {$[0.1,0.2)$} & {$[0.2,0.35)$} & {$[0.35,0.5)$} & {$[0.5,0.75)$} & {$[0.75,1.25)$} & {$[1.25,2.0)$} & {$[2.0, \infty)$} \\
\hline$[-\infty, 0.05)$ & 0.004 & 0.005 & 0.002 & 0.001 & 0.001 & 0.000 & 0.000 & 0.000 & 0.000 \\
\hline$[0.05,0.1)$ & 0.008 & 0.012 & 0.009 & 0.004 & 0.002 & 0.001 & 0.001 & 0.000 & 0.000 \\
\hline$[0.1,0.2)$ & 0.003 & 0.009 & 0.011 & 0.009 & 0.005 & 0.003 & 0.002 & 0.001 & 0.000 \\
\hline$[0.2,0.35)$ & 0.001 & 0.005 & 0.012 & 0.016 & 0.016 & 0.009 & 0.005 & 0.002 & 0.001 \\
\hline$[0.35,0.5)$ & 0.001 & 0.004 & 0.013 & 0.033 & 0.046 & 0.035 & 0.018 & 0.008 & 0.004 \\
\hline$[0.5,0.75)$ & 0.001 & 0.002 & 0.008 & 0.026 & 0.048 & 0.050 & 0.034 & 0.017 & 0.007 \\
\hline$[0.75,1.25)$ & 0.002 & 0.001 & 0.005 & 0.015 & 0.034 & 0.052 & 0.053 & 0.038 & 0.016 \\
\hline$[1.25,2.0)$ & 0.009 & 0.002 & 0.004 & 0.016 & 0.034 & 0.064 & 0.107 & 0.108 & 0.056 \\
\hline$[2.0, \infty)$ & 0.016 & 0.002 & 0.006 & 0.021 & 0.039 & 0.058 & 0.091 & 0.123 & 0.089 \\
\hline
\end{tabular}

Table 5.4: Uncertainties on the unfolding matrix. The bins of $\mathrm{Q}_{\mathrm{QE}}^{2}$ are in units of $\mathrm{GeV}^{2}$.

\subsubsection{Efficiency and Acceptance Corrections}

After the signal distribution has had relevant backgrounds subtracted and has been corrected for detector smearing effects, acceptances and efficiencies must be addressed. An overall bin by bin correction for this, the product of acceptance and efficiency, is made. This product is referred to as the efficiency henceforth.

We use the simulation to find the efficiency correction, which is given by Equation 5.3 discussed above. We use the efficiency as a function of $\mathrm{Q}_{\mathrm{QE}}^{2}$ when we apply the efficiency correction.

Signal events are lost for several reasons. Some losses are due to reconstruction failures such as charge mis-reconstruction. Other loses are acceptance related. The requirement that a muon be matched to a track in MINOS removes events at higher angles, with efficiency generally declining with increasing angle.

We find that the acceptance is not uniform as a function of $\mathrm{Q}_{\mathrm{QE}}^{2}$. The recoil cut removes a large number of events at high $\mathrm{Q}_{\mathrm{QE}}^{2}$. Particularly large losses occur for low neutrino energy events with high $\mathrm{Q}_{\mathrm{QE}}^{2}$. See Figure 5.17 which is populated by a large signal only Monte Carlo sample. Figure 5.17 contains plots that show the average angle for different bins of neutrino energy and $\mathrm{Q}_{\mathrm{QE}}^{2}$ for all true CCQE events generated within the fiducial volume and for those same events that pass the analysis selection cuts. For neutrinos with more than $3 \mathrm{GeV}$ in energy, the average 

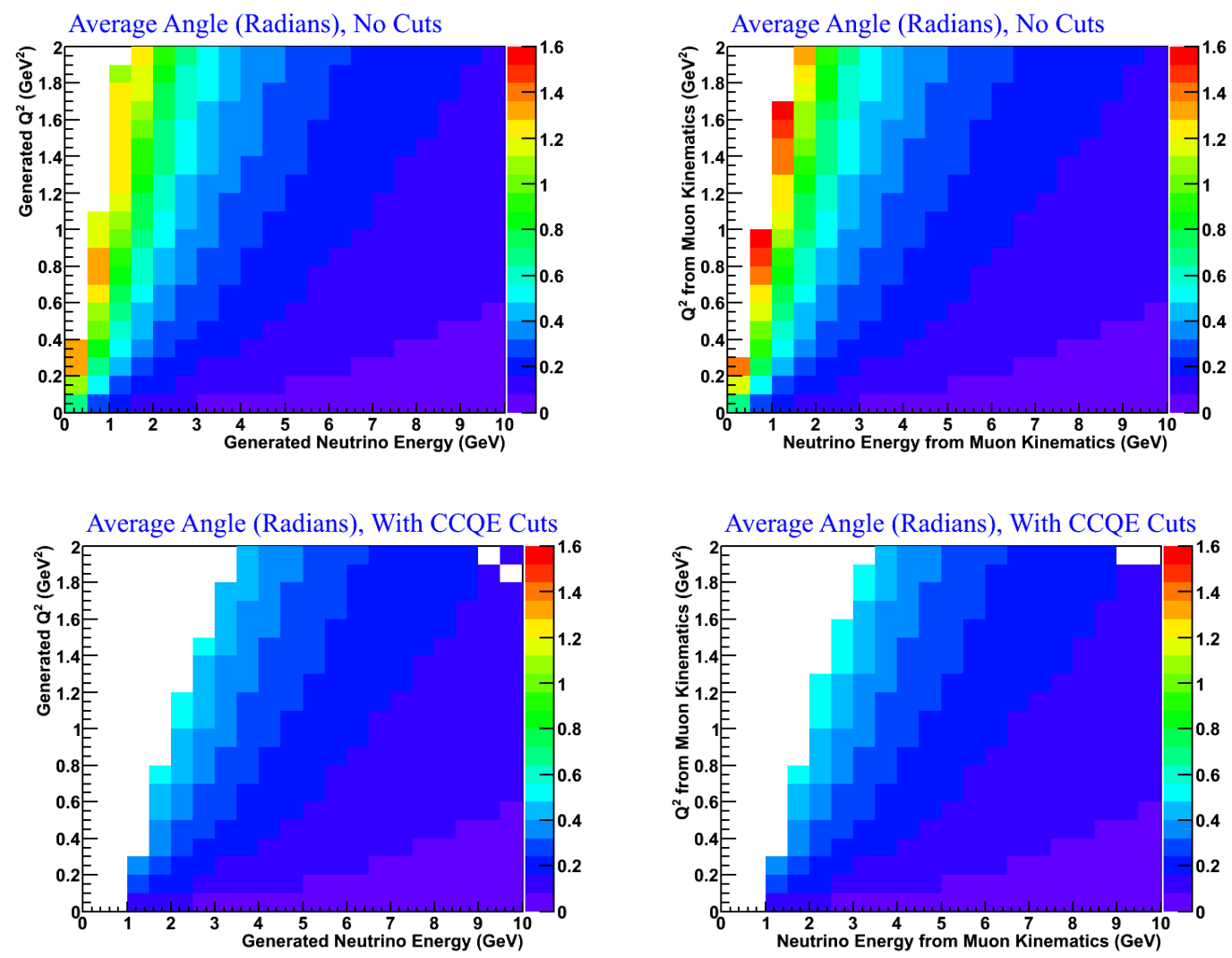

Figure 5.17: The average angle for bins of $\mathrm{Q}_{\mathrm{QE}}^{2}$ and neutrino energy before and after analysis cuts. Two sets of plots are shown, ones that use generator level values and others that use the true muon kinematics to find $\mathrm{Q}_{\mathrm{QE}}^{2}$ and neutrino energy.

angle shows little change after applying analysis cuts. This cut includes requiring the event be matched to a muon in MINOS. Due to the minimum energy required to form a track in MINOS and to traverse the ECAL and HCAL regions of MINER $\nu \mathrm{A}$, the match requirement cuts all events below $1 \mathrm{GeV}$ irrespective of the angle of the final state muon. We find events with energies between 1 and $3 \mathrm{GeV}$ experience the greatest amount of shaping. All bins with average angles greater than about 0.5 radians are lost.

To further understand which events are lost, we plot the expected distribution of 
neutrino energies for each bin of $\mathrm{Q}_{\mathrm{QE}}^{2}$ used in the analysis. We also look at values of $\mathrm{Q}_{\mathrm{QE}}^{2}$ greater than $2.0 \mathrm{GeV}^{2}$, which are not included in the cross-section result. See Figures 5.18, 5.19, 5.20, 5.21, and 5.22 for these plots. We find similar results as Figure 5.17. Above $3 \mathrm{GeV}$ of neutrino energy, minor angular acceptance effects are present. We see the most dramatic effect for the $\mathrm{Q}_{\mathrm{QE}}^{2}>2 \mathrm{GeV}^{2}$ bin. Many of the events we lose in the $2-3 \mathrm{GeV}$ region are in this bin, which we do not report as part of the cross-section result. We do see some noticable efficiency losses for the highest $\mathrm{Q}_{\mathrm{QE}}^{2}$ bins for which we report a cross-section, but find most of the sensitivity to the differential cross-section in that region comes from higher neutrino energies within the analysis. For those higher energies, we do not see much acceptance loss. Since for higher neutrino energies the muon is relativistically boosted, higher angle events will appear as lower angle events giving this analysis additional sensistivity than lower neutrino cross-section measurements. If there are issues of modeling angle for higher muon angles, we do not expect it to greatly impact this analysis.

Other effects that reduce the efficiency are accounted for as a flat reduction in efficiency across all values of $\mathrm{Q}_{\mathrm{QE}}^{2}$ considered in this analysis. A study was performed to establish the product of MINER $\nu \mathrm{A}$ tracking efficiency and the efficiency of matching an exiting muon to a MINOS track. Another study was performed to establish how frequently the reconstruction used by MINOS failed to reconstruct a track corresponding to a muon traversing the MINOS detector. Other events are lost due to deadtime. Deadtime can prevent a signal event from being reconstructed in the fiducial volume or from being matched correctly to MINOS. Last, some signal events are lost by the deadtime cut described in Section 5.1.2. None of these effects are accounted for in the Monte Carlo. The flat efficiency corrections are already applied in efficiency corrected distributions shown in Figure 5.9. Table 5.5 shows 

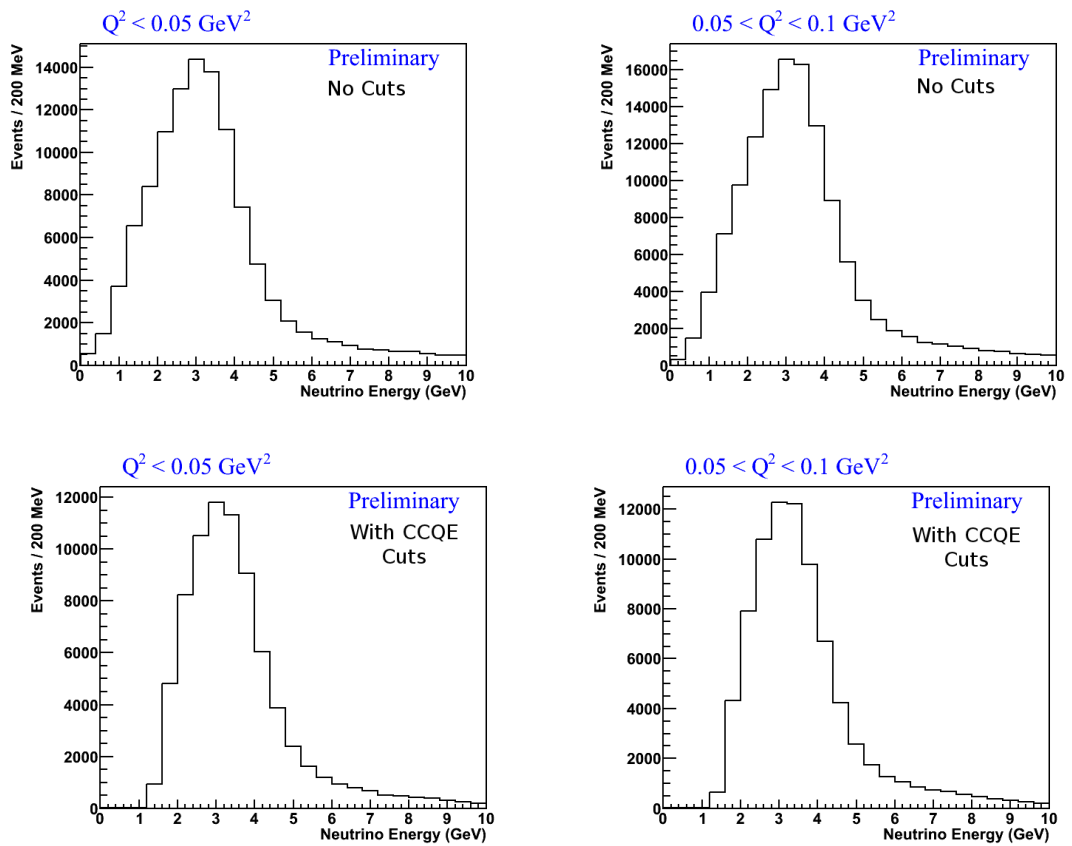

Figure 5.18: Distributions of events generated within the fiducial volume before and after analysis cuts.
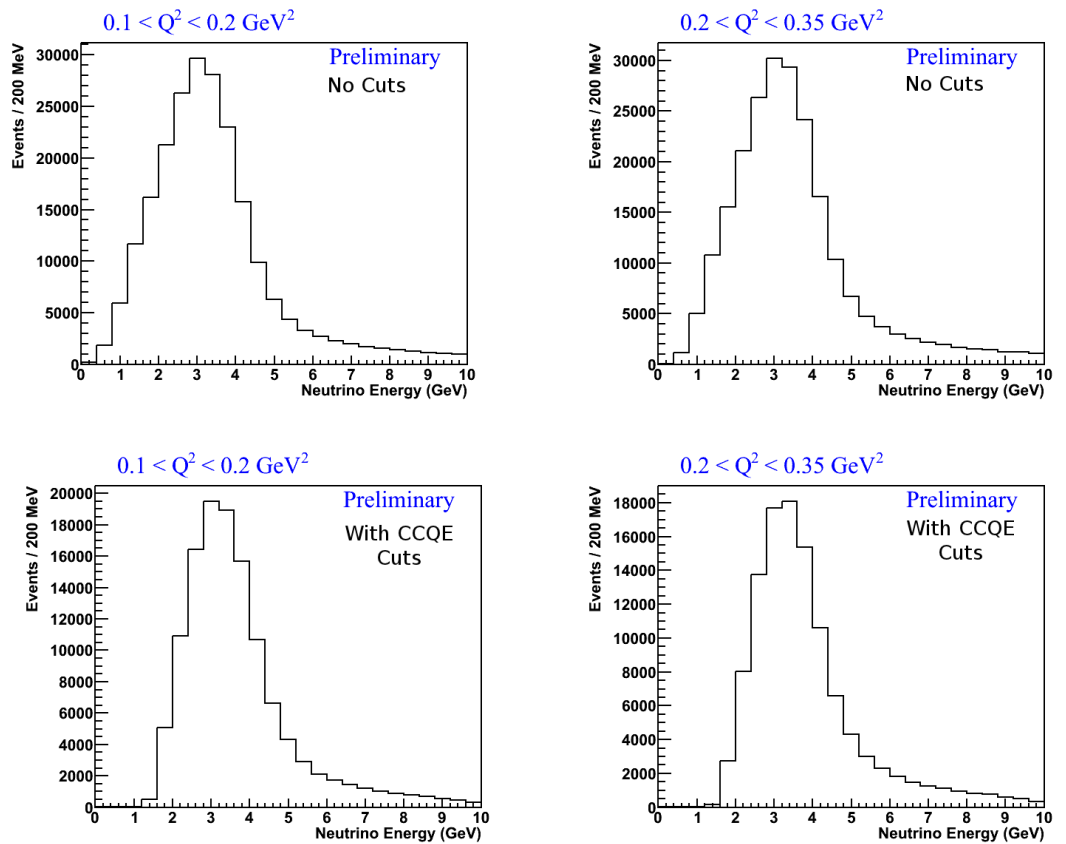

Figure 5.19: Distributions of events generated within the fiducial volume before and after analysis cuts. 

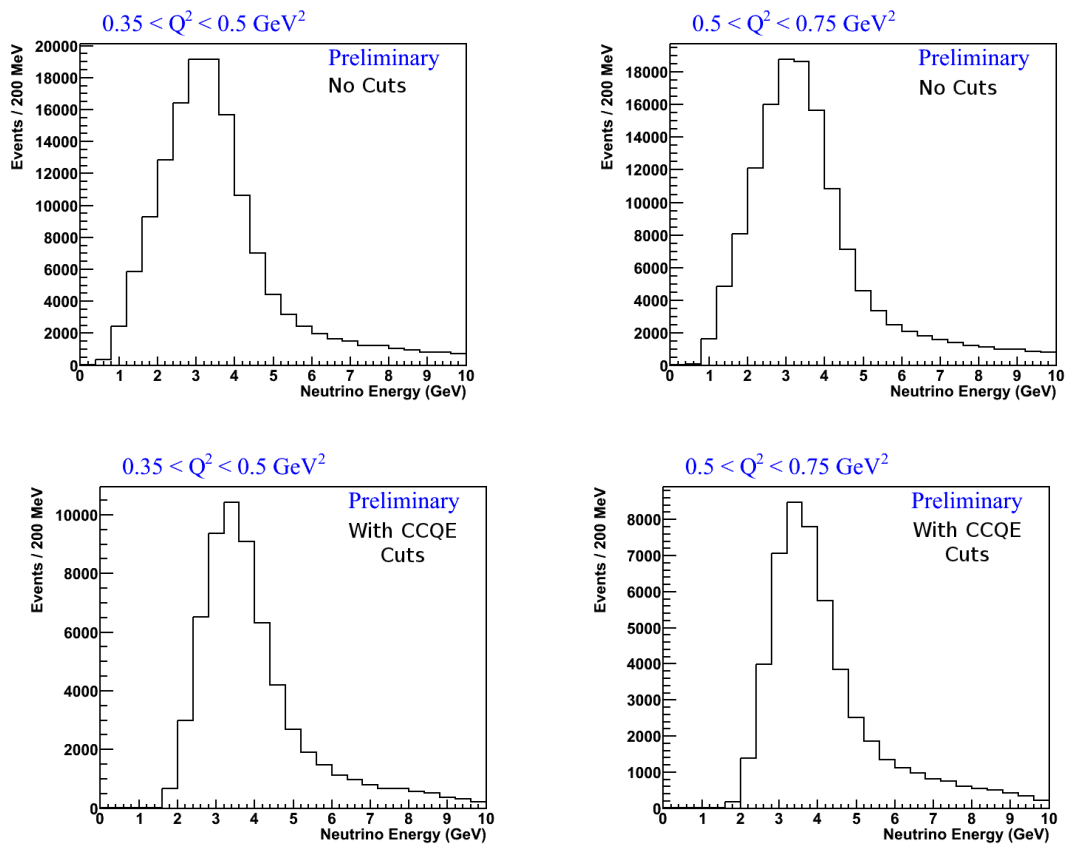

Figure 5.20: Distributions of events generated within the fiducial volume before and after analysis cuts.
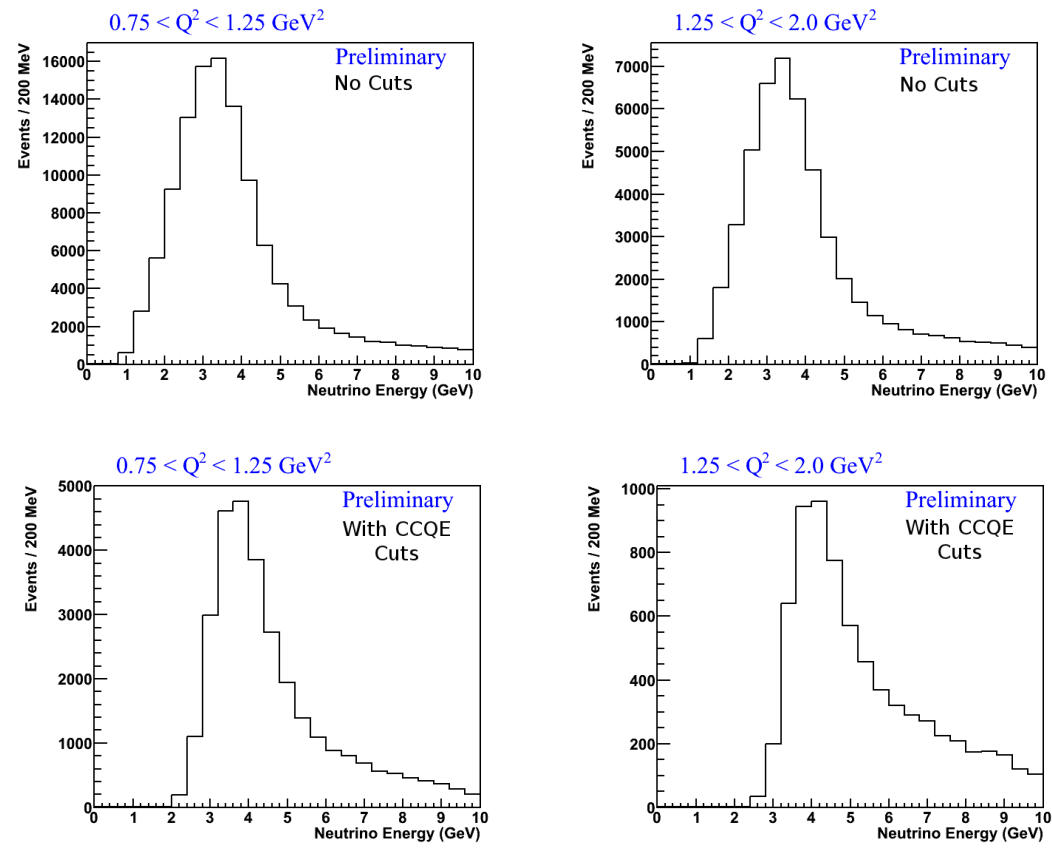

Figure 5.21: Distributions of events generated within the fiducial volume before and after analysis cuts. 

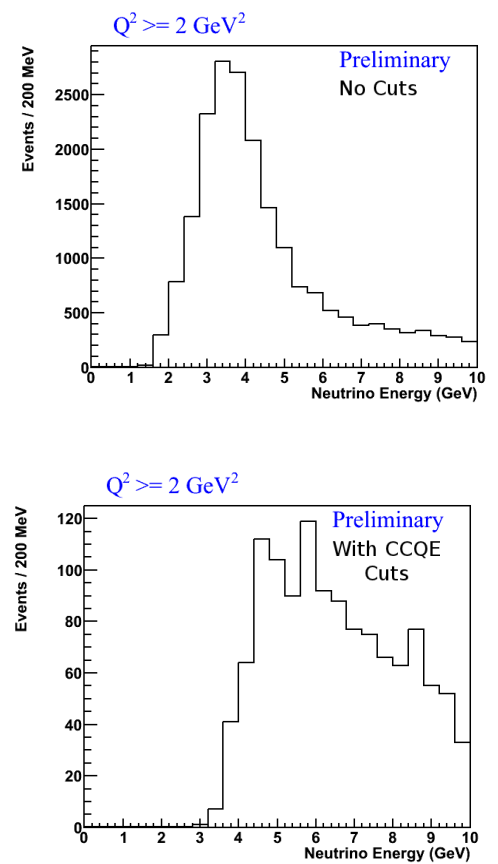

Figure 5.22: Distributions of events generated within the fiducial volume before and after analysis cuts.

\begin{tabular}{c|c}
\hline \hline Type of Efficiency & Correction \\
\hline Tracking $\times$ MINOS Matching Efficiency & $0.966 \pm 0.025$ \\
MINOS Tracking Efficiency & $0.985 \pm 0.003$ \\
Efficiency After Deadtime Effects & $0.99 \pm 0.01$ \\
Efficiency of Rock Muon Cut on Deadtime & $0.995 \pm 0.0025$ \\
\hline Total & $0.937 \pm 0.0272$ \\
\hline \hline
\end{tabular}

Table 5.5: Flat selection efficiencies not accounted for in the simulation.

the flat efficiencies described above and Table 5.6 lists the efficiencies for each bin of $\mathrm{Q}_{\mathrm{QE}}^{2}$.

The $\mathrm{Q}_{\mathrm{QE}}^{2}$ distribution with background subtraction, unfolding, and efficiency correction applied can be seen in Figure 5.23. 


\begin{tabular}{c|c}
\hline \hline $\mathrm{Q}_{\text {True }}^{2}\left(\mathrm{GeV}^{2}\right)$ & Efficiency $\times$ Acceptance \\
\hline$[0,0.05)$ & 0.646 \\
{$[0.05,0.1)$} & 0.582 \\
{$[0.1,0.2)$} & 0.513 \\
{$[0.2,0.35)$} & 0.456 \\
{$[0.35,0.5)$} & 0.401 \\
{$[0.5,0.75)$} & 0.328 \\
{$[0.75,1.25)$} & 0.232 \\
{$[1.25,2.0)$} & 0.126 \\
{$[2.0, \infty)$} & 0.054 \\
\hline \hline
\end{tabular}

Table 5.6: Product of efficiency and acceptance for each bin of $\mathrm{Q}_{\mathrm{QE}}^{2}$.
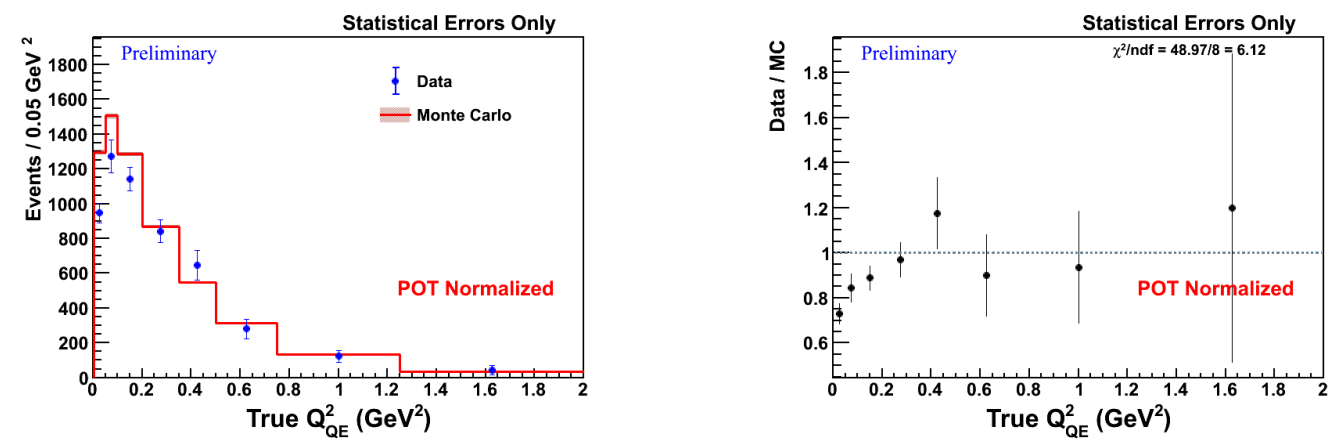

Figure 5.23: The $\mathrm{Q}_{\mathrm{QE}}^{2}$ distribution with background subtraction, unfolding, and efficiency corrections applied. Data/MC ratios each with a $\chi^{2}$ value showing the consistency of the ratio with a flat line are also shown. 


\subsubsection{Flux}

We discuss how we estimate the flux with the simulation in Section 4.2.1 and the reweighting procedure we use to correct for mis-modeling of that flux in Section 4.2.2. The muon anti-neutrino flux used in this analysis can be found in Figure 4.14. For ranges of $\mathrm{x}_{\mathrm{F}}$ of primary pions and kaons that cannot be well constrained by external data sets, spreads of various models lead to an overall systematic error that affects the uncertainty on the normalization for the sample in this analysis. We discuss this procedure in Section 5.3.5.

After all reweighting described above has been applied to the simulated NuMI flux, we find for muon anti-neutrinos with less than $12 \mathrm{GeV}$ of energy an intergrated flux of $2.71951 \cdot 10^{-8} \mathrm{~cm}^{-2} /$ POT. Multipling by the total exposure of $8.96146 \cdot 10^{19}$ POT for all data used in this analysis, we find an integrated flux of $\Phi=2.43708 \cdot 10^{12}$ $\mathrm{cm}^{-2}$.

\subsubsection{Number of Target Protons}

To find the differential cross-section, the total number of protons within the volume must be known. Although the fiducial volume for this analysis is composed primarily of polystyrene scintillator $(\mathrm{CH})$, other elements are present. A description of this is given in Section 3.5.3. In total, we estimate that each plane contained $6.53 \cdot 10^{23}$ protons per $\mathrm{cm}^{2}$ per plane. Therefore the total fiducial volume contains $7.027 \cdot 10^{29}$ target protons. We find an overall uncertainty of $1.4 \%$ on the number of protons.

The differential cross-section $\frac{\mathrm{d} \sigma}{\mathrm{d} \mathrm{Q}_{\mathrm{QE}}^{2}}$ found after the normalizations from number of target protons and integrated flux is shown in Figure 5.24. 

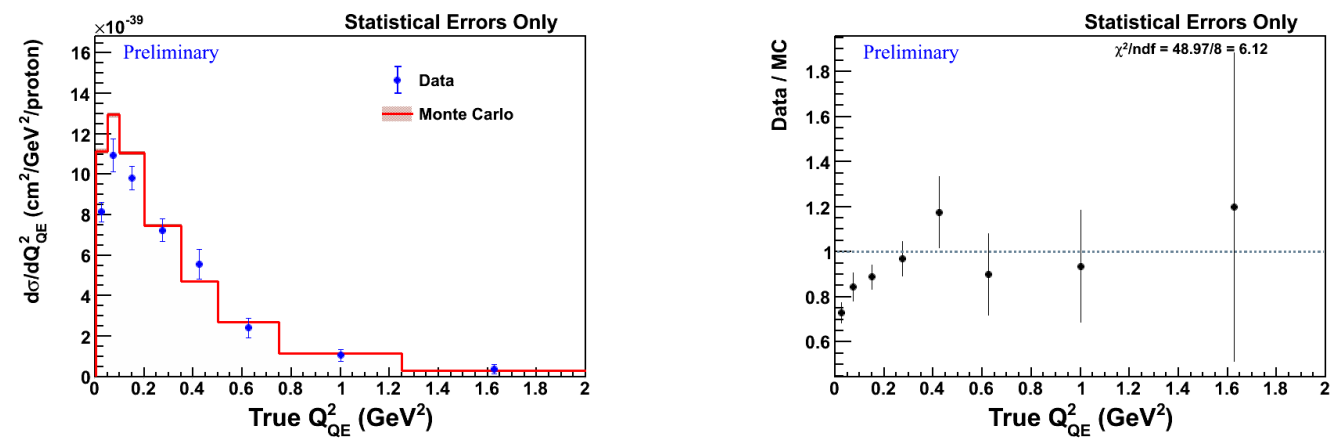

Figure 5.24: The differential cross-section $\frac{\mathrm{d} \sigma}{\mathrm{d} \mathrm{Q}_{\mathrm{QE}}^{2}}$ extracted on $\mathrm{CH}$ in MINER $\nu \mathrm{A}$ with statistical errors. Data/MC ratios each with a $\chi^{2}$ value showing the consistency of the ratio with a flat line are also shown. We find a deficit of data events at low $\mathrm{Q}_{\mathrm{QE}}^{2}$ compared to simulation.

\subsection{Systematic Errors}

We employ the simulations described in Section 4.2 to predict the flux, unfold the signal sample, subtract backgrounds, and make efficiency corrections. We know we do not perfectly model these various elements that go into the analysis. We use a variety of studies to establish systematic errors to account for this. In most cases, we find the covariance matrix for a particular systematic error. We use the square root of the diagonal elements within the covariance matrices for the systematic errors.

We find the covariance matrix in three general ways. In one case, we consider a single effect in applying the systematic error. First we calculate the differential cross-section from the nominal simulation. We then recalculate the differential crosssection using a version of the simulation with a single element varied. The covariance matrix associated with that procedure is

$$
\mathrm{C}_{\mathrm{ij}}=\left(\left(\frac{\mathrm{d} \sigma}{\mathrm{dQ}_{\mathrm{QE}}^{2}}\right)_{\mathrm{Var}, \mathrm{i}}-\left(\frac{\mathrm{d} \sigma}{\mathrm{dQ}_{\mathrm{QE}}^{2}}\right)_{\mathrm{i}}\right) \cdot\left(\left(\frac{\mathrm{d} \sigma}{\mathrm{dQ}_{\mathrm{QE}}^{2}}\right)_{\mathrm{Var}, \mathrm{j}}-\left(\frac{\mathrm{d} \sigma}{\mathrm{dQ}_{\mathrm{QE}}^{2}}\right)_{\mathrm{j}}\right),
$$


where $\mathrm{C}_{\mathrm{ij}}$ is the covariance matrix. The term $\left(\frac{\mathrm{d} \sigma}{\mathrm{dQ}_{\mathrm{QE}}^{2}}\right)_{\mathrm{i}}$ is the $\mathrm{i}^{\text {th }}$ bin of the differential cross-section found in the simulation and the term $\left(\frac{\mathrm{d} \sigma}{\mathrm{d} \mathrm{Q}_{\mathrm{QE}}^{2}}\right)_{\mathrm{Var}, \mathrm{i}}$ is the $\mathrm{i}^{\text {th }}$ bin of the differential cross-section calculated after varying a particular component of the simulation. Certain systematic variations are considered in this way, such as assessing the systematic error that stems from cross-talk or the effect of ArgoNeuT.

In other cases, we consider multiple variations to the nominal simulation to understand a specific systematic error. We handle these multiple variations in two ways. In the first case we average over the covariance matrices found for each variation in this way

$$
\begin{gathered}
\mathrm{C}_{\mathrm{ij}}=\frac{1}{\mathrm{~N}} \sum_{\mathrm{k}}^{\mathrm{N}}\left(\left(\frac{\mathrm{d} \sigma}{\mathrm{d} \mathrm{Q}_{\mathrm{QE}}^{2}}\right)_{\mathrm{Var}, \mathrm{i}}^{\mathrm{k}}-\left(\frac{\mathrm{d} \sigma}{\mathrm{dQ}_{\mathrm{QE}}^{2}}\right)_{\mathrm{i}}\right) . \\
\left(\left(\frac{\mathrm{d} \sigma}{\mathrm{d} \mathrm{Q}_{\mathrm{QE}}^{2}}\right)_{\mathrm{Var}, \mathrm{j}}^{\mathrm{k}}-\left(\frac{\mathrm{d} \sigma}{\mathrm{d} \mathrm{Q}_{\mathrm{QE}}^{2}}\right)_{\mathrm{j}}\right)
\end{gathered}
$$

Here $\mathrm{k}$ is the index for a particular covariance matrix involved in the calculation of that average. Like in Equation 5.8, $\left(\frac{\mathrm{d} \sigma}{\mathrm{d} Q_{\mathrm{QE}}^{2}}\right)_{\mathrm{i}}$ is the $\mathrm{i}^{\text {th }}$ bin of the differential cross-section found in the simulation. The term $\left(\frac{\mathrm{d} \sigma}{\mathrm{dQ} Q_{\mathrm{QE}}^{2}}\right)_{\text {Var, } \mathrm{i}}^{\mathrm{k}}$ is the $\mathrm{i}^{\text {th }}$ bin of the $\mathrm{k}^{\text {th }}$ variation of the differential cross-section, where the cross-section is recalculated after varying a particular component of the simulation. This method is used to assess the systematic error for muon energy reconstruction, neutron interaction modeling, as well as other effects. 
We consider multiple variations in one additional way,

$$
\begin{aligned}
\mathrm{C}_{\mathrm{ij}}= & \frac{1}{\mathrm{~N}} \sum_{\mathrm{k}}^{\mathrm{N}}\left(\left(\frac{\mathrm{d} \sigma}{\mathrm{dQ} \mathrm{QE}_{\mathrm{QE}}^{2}}\right)_{\text {Var,i }}^{\mathrm{k}}-\left(\frac{\mathrm{d} \sigma}{\mathrm{dQ}_{\mathrm{QE}}^{2}}\right)_{\mathrm{Avg}, \mathrm{i}}\right) . \\
& \left(\left(\frac{\mathrm{d} \sigma}{\mathrm{d} \mathrm{Q}_{\mathrm{QE}}^{2}}\right)_{\text {Var }, \mathrm{j}}^{\mathrm{k}}-\left(\frac{\mathrm{d} \sigma}{\mathrm{d} \mathrm{Q}_{\mathrm{QE}}^{2}}\right)_{\mathrm{Avg}, \mathrm{j}}\right)
\end{aligned}
$$

Equation 5.10 is similar to Equation 5.9 where the term $\left(\frac{\mathrm{d} \sigma}{\mathrm{d} \mathrm{Q}_{\mathrm{QE}}^{2}}\right)_{\mathrm{Var}, \mathrm{i}}^{\mathrm{k}}$ is the $\mathrm{i}^{\text {th }}$ bin of the $\mathrm{k}^{\text {th }}$ variation of the differential cross-section calculated after varying a particular component of the simulation. However, instead of using $\left(\frac{\mathrm{d} \sigma}{\mathrm{d} Q_{\mathrm{QE}}^{2}}\right)$, the $i^{\text {th }}$ bin of the differential cross-section found in the simulation, we use $\left(\frac{\mathrm{d} \sigma}{\mathrm{d}_{\mathrm{QE}}^{2}}\right)_{\mathrm{Avg}, \mathrm{i}}$ which is the $\mathrm{i}^{\text {th }}$ bin of the average of the differential cross-section for all variations to the simulation for a particular systematic error. We use this method when determining systematic errors involving the flux.

The final method we use for establishing systematic errors is to use a flat uncertainty on the flat efficiency corrections which are discussed in Section 5.2.3.

When calculating systematic errors using Equations 5.8, 5.9, and 5.10, we repeat particular steps of the analysis depending upon the systematic error in question. If a particular source of systematic error does not impact a particular component of the analysis, that component is not repeated. Depending upon the case, we repeat the calculation of the $\mathrm{Q}_{\mathrm{QE}}^{2}$ distribution, the fit and subtraction for background, the calculation and application of the unfolding matrix, the calculation and correction for efficiency, and the normalization for the flux. We indicate each of the particular elements that are varied when discussing a particular systematic error.

For ease of interpretation, we consider different classes of systematic errors. We consider systematic errors that result from uncertainties on muon reconstruction, 
recoil reconstruction, GENIE primary and final state interaction models, hadronic interaction models, flux, and other miscillaneous effects. An examination of each follows. For the results of a particular systematic error study, see Table 5.12.

\subsubsection{Muon Reconstruction}

The final state muon we reconstruct has uncertainties on muon energy, muon angle, vertex position, and various efficiencies relating to reconstruction.

\section{Muon Energy Reconstruction}

Uncertainty on muon energy has several sources.

- Uncertainty on the amount of material present in MINER $\nu \mathrm{A}$

- Uncertainty on the energy loss $\left(\frac{\mathrm{dE}}{\mathrm{dx}}\right)$ model

- Uncertainty on MINOS energy reconstruction

- Uncertainty stemming from energy loss in ArgoNeuT

Reconstruction uncertainty from the amount of material and the $\frac{\mathrm{dE}}{\mathrm{dx}}$ model is due to the manner in which muon energy is reconstructed, a description of which can be found in Section 4.3.7. From a study, an uncertainty of $11 \mathrm{MeV}$ and $30 \mathrm{MeV}$ is assessed due to the amount of material and the $\frac{\mathrm{dE}}{\mathrm{dx}}$ model, respectively.

Energy reconstruction in MINOS has an uncertainty dependent upon the manner in which the particle is reconstructed and what energy it is found to have. In each of the following cases, a percentage uncertainty is applied to the energy reconstructed in MINOS. If the muon is reconstructed by range, an uncertainty of $2 \%$ is applied, as found by MINOS [87]. If the muon is reconstructed by curvature, but has a 
momentum reconstructed in MINOS of $1.5 \mathrm{GeV} / \mathrm{c}$ or more, an uncertainty of $2.82 \%$ is applied. Last, if the muon is reconstructed by curvature, but has a momentum reconstructed in MINOS of less than $1.5 \mathrm{GeV} / \mathrm{c}$, an uncertainty of $5.39 \%$ is applied. We find the errors on energy reconstructed by curvature by examining events that have momentum successfully reconstructed in MINOS by both curvature and range. See Figure 5.25 for the difference of the inverse of momentum reconstructed by range and curvature. A fit to a Guassian is performed for this Figure. We then look at differences in data and simulation for this figure to find a systematic error. Repeating the analysis for different muons for different momenta, we find energy dependent errors listed above.

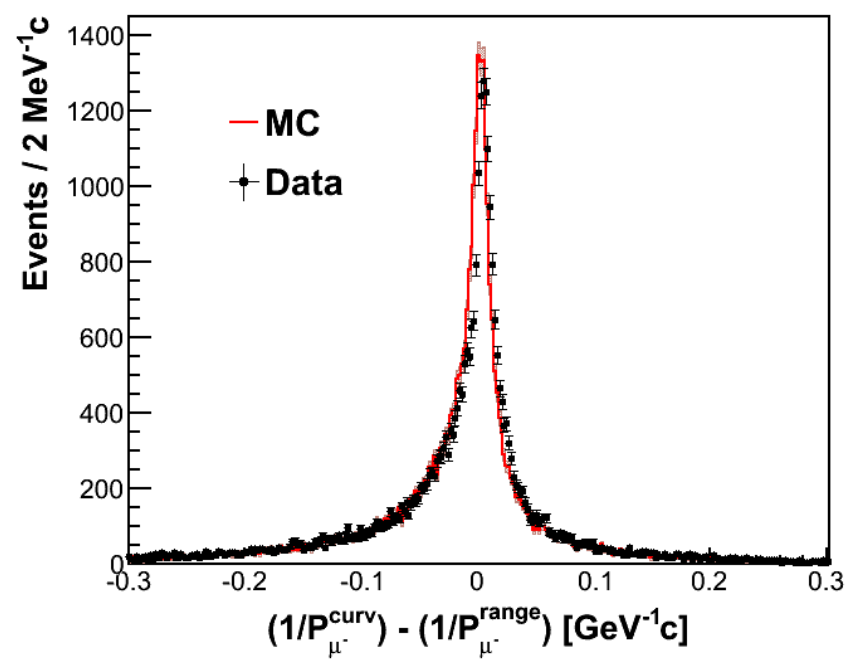

Figure 5.25: The difference of the inverses of momentum found in MINOS by range and curvature. This plots is by G. Diaz of the MINER $\nu$ A collaboration.

The uncertainty on muon energy reconstruction is propagated as an uncertainty on the differential cross-section by increasing or decreasing the muon energy based on the sum of the above uncertainties. A conservative approach is used instead of adding these uncertainties in quadrature. The unfolding matrix and $\mathrm{Q}_{\mathrm{QE}}^{2}$ for the 
event is then calculated and the differential cross-section is then extracted. The systematic error is then assessed using Equation 5.9.

To assess a systematic effect from ArgoNeuT, we assume a $20 \%$ uncertainty on the mass on the ArgoNeuT simulation described in Section 4.2.5. To find the systematic error, we rerun the entire simulation chain without the simulation of ArgoNeuT in place. We remeasure the efficiency and unfolding matrix and then remeasure the differential cross-section. We then take $20 \%$ of the uncertainty found using Equation 5.8 to be the systematic error. Since the unfolding procedure should remove most of the bias in reconstructed $\mathrm{Q}_{\mathrm{QE}}^{2}$ stemming from the presence of ArgoNeuT, we take only a fraction of the error reflecting uncertainty on the mass of ArgoNeuT instead of the full $100 \%$.

\section{Muon Angle Reconstruction}

To find the systematic error for reconstructed muon angle, we use a study that involves breaking tracks and remeasuring angles. This study took MINOS matched muons with less than $20 \mathrm{GeV}$ of energy reconstructed within the fiducial volume of MINER $\nu \mathrm{A}$ and broke them into two pieces. Both tracks use the cluster present at the break point of the track. Tracking is repeated and the angle at the break point for both tracks is compared. Figure 5.26 shows the residuals of the two components of the track for data and the simulation. Differences in resolutions between data and the simulation are below 1 milliradian.

To ensure we fully account for even this very low level of disagreement, we apply an angular uncertainty of 2 milliradians. We apply this uncertainty by smearing the angle by randomly sampling from a Gaussian distribution with a standard deviation of 2 milliradians. After this variation has been made, we then recalculate $\mathrm{Q}_{\mathrm{QE}}^{2}$ 

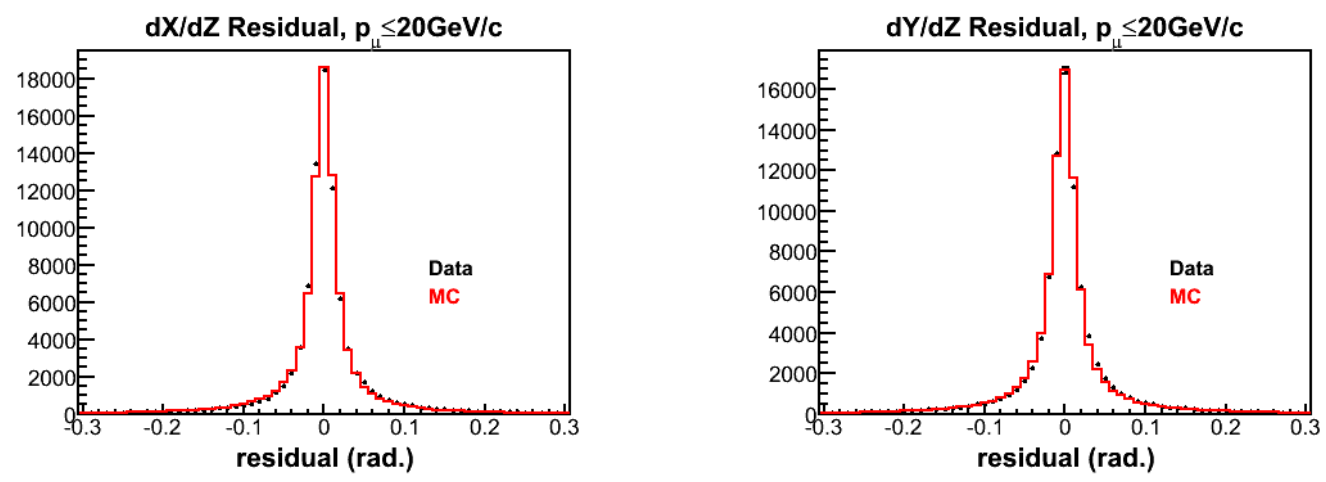

Figure 5.26: Data and simulation overlays for the angle residuals of two pieces of a broken track. The residuals for the angles in the $\mathrm{XZ}$ and $\mathrm{YZ}$ planes are found. These plots are made by $\mathrm{B}$. Ziemer of the MINER $\nu$ A collaboration.

and the unfolding matrix and extract the resulting differential cross-section. The systematic error is assessed using Equation 5.8.

\section{Muon Vertex Reconstruction}

We apply a systematic error for vertex reconstruction using the same broken track study described above. The vertex at the overlap point of the two track pieces is compared. Data and simulation overlays for $\mathrm{X}$ and $\mathrm{Y}$ vertex residuals can be found in Figure 5.27. Data and simulation agree at roughly the $1 \mathrm{~mm}$ level in both the $\mathrm{X}$ and $\mathrm{Y}$ directions. In the lateral direction, comparisons are harder to make. We assume a conservative resolution uncertainty of $1 \mathrm{~cm}$.

We then apply the vertex smearing systematic by randomly sampling from a Gaussian distribution with a standard deviation of $1 \mathrm{~mm}$ in the $\mathrm{X}$ and $\mathrm{Y}$ directions and $1 \mathrm{~cm}$ in the $\mathrm{Z}$ direction. This variation should only affect the efficiency correction which is recalculated along with the differential cross-section. The systematic error is found using Equation 5.8. 

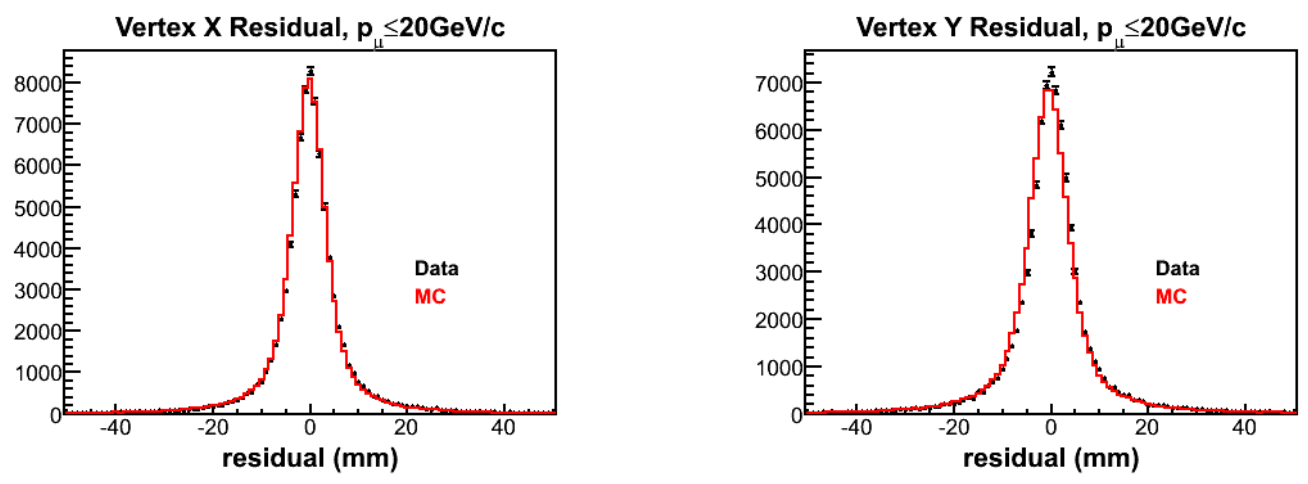

Figure 5.27: Data and simulation overlays for position residuals of two pieces of a broken track. The residuals for $\mathrm{X}$ and $\mathrm{Y}$ vertices are found. These plots are made by B. Ziemer of the MINER $\nu \mathrm{A}$ collaboration.

\section{Efficiency of Finding a Track in MINER $\nu$ A and Matching it to a MINOS} Track

The product of the efficiency of finding a track in MINER $\nu \mathrm{A}$ and the efficiency of matching that to a track in MINOS is found by a study using tracks first found in MINOS. The MINOS track must start in the first MINOS plane, must point back to the beginning of the first plane of the MINER $\nu \mathrm{A}$ tracker, and have a sufficient amount of energy in different regions of MINER $\nu \mathrm{A}$. The efficiency of finding a track in MINER $\nu \mathrm{A}$ that matches with the MINOS track used in this study is the above product. Data and simulation overlays for this product as a function of momentum can be found in Figure 5.28.

The product of the efficiency of finding a track in MINER $\nu \mathrm{A}$ and the efficiency of matching that to a track in MINOS is found to be $3.4 \%$ lower in data than in Monte Carlo. This is also described in Section 5.2.3. We find an uncertainty on this correction of $2.5 \%$. This value is applied directly as a systematic error on the differential cross-section. 


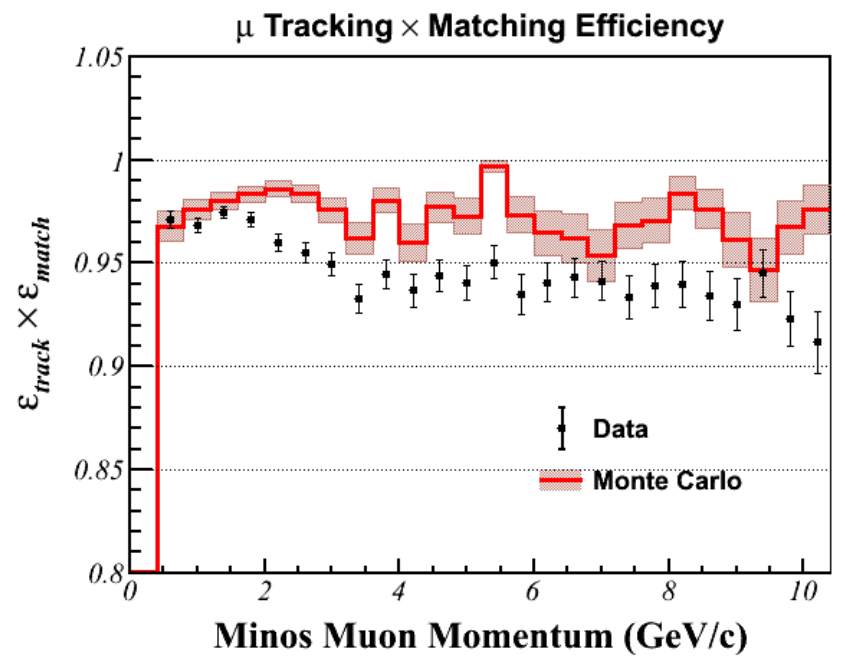

Figure 5.28: Data and simulation overlays of the product of MINER $\nu$ A tracking efficiency and MINOS matching efficiency as a function of muon momentum in MINOS. We find the matching to be lower in data than in the simulation. This plot was made by A. Fiorentini of the MINER $\nu \mathrm{A}$ collaboration.

\section{Deadtime Cut on Rock Muon Background}

A description of this veto is given in Section 5.1.2. An efficiency correction to account for lost signal events resulting from this cut is described in Section 5.2.3. This scan found 4 out of 653 signal events to be rock muon background. We take the statistical uncertainty on the number of identified rock muon events to yield a $50 \%$ uncertainty on the value of the efficiency correction. This gives a $0.25 \%$ systematic uncertainty on the $0.55 \%$ flat efficiency correction.

\section{MINOS Tracking Efficiency}

The tracking efficiency must be considered in MINOS as well as in MINER $\nu$ A. To estimate the tracking efficiency, an alternative pattern recognition scheme to the MINOS tracking is used. Using MINOS data, we search for continuous strings of hits associated with a MINER $\nu \mathrm{A}$ track specifically in cases where a MINOS track is 
not found. We refer to these as stubs. We search for stubs longer than the minimum tracking threshold in MINOS, which we presume to be unreconstructed tracks. We then compare the number of expected and found tracks which we use to estimate the number of lost tracks in data and simulation. We find the percentage of lost tracks to be $2.32 \pm 0.18 \%$ in data and $0.79 \pm 0.05 \%$ in the simulation. The difference between the two is $1.5 \%$. To be conservative, we boost the statistical error on this number by $50 \%$ to get a $0.3 \%$ systematic error on this result. Thus we find the MINOS tracking efficiency is $1.5 \% \pm 0.3 \%$ lower in data than in MC. A flat efficiency correction is made for this as discussed in Section 5.2.3. The $0.3 \%$ error on this correction is applied directly to the differential cross-section, fully correlated across $Q_{Q \mathrm{QE}}^{2}$.

\section{MINOS Acceptance}

To ensure that we appropriately assess a systematic error for acceptance of events in MINOS, we divide MINOS into four quadrants and remeasure the differential crosssection for each (see Figure 5.29). We find in the lowest bin of $\mathrm{Q}_{\mathrm{QE}}^{2}$ for the quadrant that covers the region of MINOS with the magentic coil a value much higher than the other three quadrants. All other bins of $\mathrm{Q}_{\mathrm{QE}}^{2}$ for each quadrant agree within statistical errors.

To apply a systematic error, we calculate the p-value for the consistency of entries within the quadrant with the coil to entries in the other quadrants for each bin of $\mathrm{Q}_{\mathrm{QE}}^{2}$. These values are listed in Table 5.7. We then find what acceptance change would be needed to give a $\mathrm{p}$-value consistent with a one sigma deviation $(\mathrm{p}>0.159)$. Roughly $40 \%$ of events in the lowest $\mathrm{Q}_{\mathrm{QE}}^{2}$ bin are in the MINOS quadrant containing the coil. Additionaly, a roughly $40 \%$ change in acceptance in that quadrant would be necessary to accout for this discrepancy. Consequently, we apply a $15 \%$ systematic 


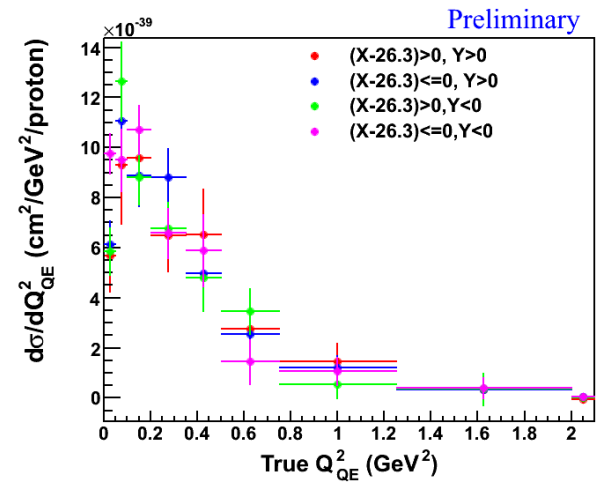

Figure 5.29: An overlay of the differential cross-section $\frac{\mathrm{d} \sigma}{\mathrm{dQ}_{\mathrm{QE}}^{2}}$ calculated for events in four different quadrants of MINOS. The boundaries of the quadrants are listed on the plot. Discrepancies in the first $\mathrm{Q}_{\mathrm{QE}}^{2}$ bin lead to an increased systematic error in that bin.

\begin{tabular}{c|ccccccccc}
\hline \hline $\mathrm{Q}_{\mathrm{QE}}^{2}$ Bin & {$[0,0.05)$} & {$[0.05,0.1)$} & {$[0.1,0.2)$} & {$[0.2,0.35)$} & {$[0.35,0.5)$} & {$[0.5,0.75)$} & {$[0.75,1.25)$} & {$[1.25,2.0)$} & {$[2.0, \infty)$} \\
\hline $\mathrm{p}-\mathrm{value}$ & 0.01 & 0.505 & 0.411 & 0.761 & 0.359 & 0.915 & 0.553 & 0.430 & 0.348 \\
\hline \hline
\end{tabular}

Table 5.7: Listed are the p-values from comparing events in the quadrant with the MINOS coil with values in the other three quadrants for each bin of $\mathrm{Q}_{\mathrm{QE}}^{2}$.

error in the first $\mathrm{Q}_{\mathrm{QE}}^{2}$ bin.

\subsubsection{GENIE and Final State Interaction Modeling Errors}

Since we rely on the simulation for background subtraction, efficiency corrections, and unsmearing, modeling inaccuracies contribute systematic error to the final result. Modeling of CCQE scattering, background processes, and final state interactions all contribute to the overall systematic error on the differential cross-section measurement. A discussion of each follows below.

\section{CCQE Modeling}

This analysis has a very small dependence on how the signal interaction is modeled in the simulation. Nominally, this effect should be removed by the unfolding procedure, 


\begin{tabular}{c|ccc}
\hline \hline $\mathrm{E}_{\bar{\nu}_{\mu}}$ & $<2 \mathrm{GeV}$ & $2-6 \mathrm{GeV}$ & $>6 \mathrm{GeV}$ \\
\hline Weight & 0.8 & $0.8+0.4 *\left(\mathrm{E}_{\bar{\nu}_{\mu}}-2 \mathrm{GeV}\right)$ & 1.2 \\
\hline \hline
\end{tabular}

Table 5.8: Weights to apply to background to find a systematic error to account for potential neutrino energy dependence of backgrounds.

but due to potential non-linear variations within a bin of $\mathrm{Q}_{\mathrm{QE}}^{2}$, a small dependence can remain. To assess the systematic error for this effect, Monte Carlo signal events are reweighted to match the signal shape in data. We then re-extract the unfolding matrix as well as efficiency corrections. We then find the systematic error using Equation 5.8.

\section{Variations of Backgrounds with Neutrino Energy}

We perform template fits of background distributions in $\mathrm{MC}$ to data in bins of $\mathrm{Q}_{\mathrm{QE}}^{2}$ as described in Section 5.2.1. However, the value of $\mathrm{Q}_{\mathrm{QE}}^{2}$ calculated for background is correlated with neutrino energy. We now consider systematic error that arises if the background is directly dependent on neutrino energy instead of $\mathrm{Q}_{\mathrm{QE}}^{2}$. To assess this, we reweight $\mathrm{MC}$ events based on the true neutrino energy. The weights, which are based on $\mathrm{Q}_{\mathrm{QE}}^{2}$ dependence of backgrounds observed in an earlier stage of the analysis, are given in Table 5.8. Figure 5.30 shows scatter plots of neutrino energy versus $Q_{\mathrm{QE}}^{2}$ and neutrino energy versus $W$. We repeat background subtraction after reweighting the background and re-extract the differential cross-section. We then find the systematic error due to this effect using Equation 5.8.

\section{GENIE Background and Final State Interaction Models}

Many GENIE interaction models and the models of Final State Interactions have established errors. To find the systematic error stemming from these models, param- 

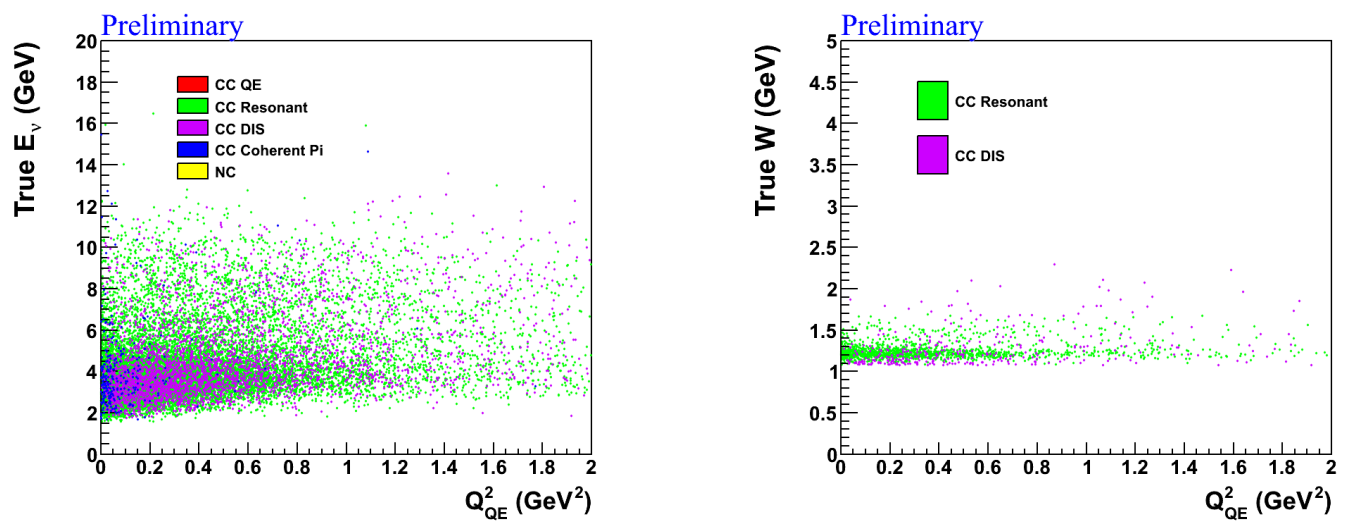

Figure 5.30: Neutrino energy versus $\mathrm{Q}_{\mathrm{QE}}^{2}$ and $\mathrm{W}$ (invariant mass of the hadronic system) versus $\mathrm{Q}_{\mathrm{QE}}^{2}$ for backgrounds events in this analysis.

eters within those models are varied up and down by one standard deviation. Events that feed into the differential cross-section are then reweighted based on the results of these variations. Changes to the GENIE models affect predicted background levels. In addition to reweighting events in that case, we repeat background subtraction as well. Changes to Final State Interaction models can alter background levels and predicted efficiencies. In this case, events are reweighted and the background subtraction and efficiency corrections steps are repeated. A table of all GENIE and Final State Interaction model variations for which we find a systematic error and the size of the model uncertainy for each is given in Table 5.9. For the variation known as Theta_Delta2Npi, the final state distribution for delta decays is reweighted from a isotropic decay distribution to a distribution found in data [109, 110]. For all variations, the differential cross-section is then remeasured so that the systematic error can be found using Equation 5.9. 


\begin{tabular}{|c|c|c|}
\hline GENIE Model Parameters & Abbreviation & $1 \sigma$ \\
\hline CC Resonance Normalization & NormCCRes & $\pm 20 \%$ \\
$\mathrm{M}_{\mathrm{A}}$ (Resonance Production) & MaRES & $\pm 20 \%$ \\
$\mathrm{M}_{\mathrm{V}}$ (Resonance Production) & MvRES & $\pm 10 \%$ \\
$\mathrm{M}_{\mathrm{A}}$ for NC Elastic Scattering & MaNCEL & $\pm 25 \%$ \\
Single $\pi$ Production from $\nu_{\mu}$ p non-resonant interactions & Rvp1pi & $\pm 50 \%$ \\
Single $\pi$ Production from $\bar{\nu}_{\mu}$ n non-resonant interactions & Rvn1pi & $\pm 50 \%$ \\
Two $\pi$ Production from $\bar{\nu}_{\mu}$ p non-resonant interactions & Rvp2pi & $\pm 50 \%$ \\
Two $\pi$ Production from $\nu_{\mu}$ n non-resonant interactions & Rvn2pi & $\pm 50 \%$ \\
\hline \hline Final State Interaction Model Parameters & Abbreviation & $1 \sigma$ \\
\hline$\pi$ Mean Free Path & MFP_pi & $\pm 20 \%$ \\
Nucleon Mean Free Path & MFN_N & $\pm 20 \%$ \\
$\pi$ Absorption & FrAbs_pi & $\pm 30 \%$ \\
$\pi$ Charge Exchange & FrCEx_pi & $\pm 50 \%$ \\
$\pi$ Elastic Scattering & FrElas_pi & $\pm 10 \%$ \\
$\pi$ Inelastic Scattering & FrInel_pi & $\pm 40 \%$ \\
$\pi$ Production & FrProd_pi & $\pm 50 \%$ \\
Nucleon Absorption & FrElas_N & $\pm 20 \%$ \\
Nucleon Charge Exchange & FrElas_N & $\pm 50 \%$ \\
Nucleon Elastic Scattering & FrElas_N & $\pm 30 \%$ \\
Nucleon Inelastic Scattering & FrInel_N & $\pm 40 \%$ \\
Nucleon Production & FrProd_N & $\pm 20 \%$ \\
Resonance Decay Branching Ratio to Photon & AGKYxF1pi & $\pm 20 \%$ \\
Delta Decay Angular Distribution & Theta_Delta2Npi & $100 \%$ \\
AGKY Hadronization Model - x & RDecB1gamma & $\pm 50 \%$ \\
\hline \hline
\end{tabular}

Table 5.9: Various GENIE and Final State Interaction model parameters, abbreviations used to later refer to those terms, and the variations used to assess the systematic errors. We reweight events for the case where each parameter is increased up or down by one standard deviation. We then use Equation 5.9 to find a systematic error. For one case, Theta_Delta2Npi, an error is not available so we reweight to a distribution based on data and use that as the variation. 


\subsubsection{Geant4 Modeling Errors}

We use Geant 4 to simulate the behavior and observable activity of final state particles in the detector. We know in certain cases this simulation performs very well such as the case of muon energy loss in MINER $\nu \mathrm{A}$. Interactions of muons are well modeled, as described by Section 5.3.1, which shows that a typical uncertainty on muon energy deposited in the detector is $40 \mathrm{MeV}$. This represents a very small component of the overall energy. Other particles have larger uncertainties stemming from how well Geant4 models their behavior. We study how well we model the interactions of neutrons, pions, protons, and electrons and photons within the detector. The primary impact of these uncertainties is on the predicted recoil energy.

\section{Neutron Modeling Uncertainties}

One of the larger systematic errors for this analysis involves the simulation of the final state neutron. We find uncertainies on both the mean free path of the neutron as well as the amount of energy deposited during a neutron interaction. Both uncertainties affect the predicted amount of recoil energy.

To assess how well the mean free path is simulated, a comparison is made of the neutron path length predicted by Geant4 and external data sets. In the simulation, neutron energies and target thicknesses that match external data are used to make the comparison. Comparisons are then made of the observed and predicted crosssection. The cross-section can be used to calculate the expected mean free path for a particle. From these studies, a $\pm 10 \%$ error on neutron path length is extracted. Events are then reweighted so that the mean free path in the simulation are $10 \%$ larger or smaller than the nominal simulation. Studies placing systematic errors on the modeling of neutrons in this analysis use data from Zanelli et al. [111], Ibaraki et 

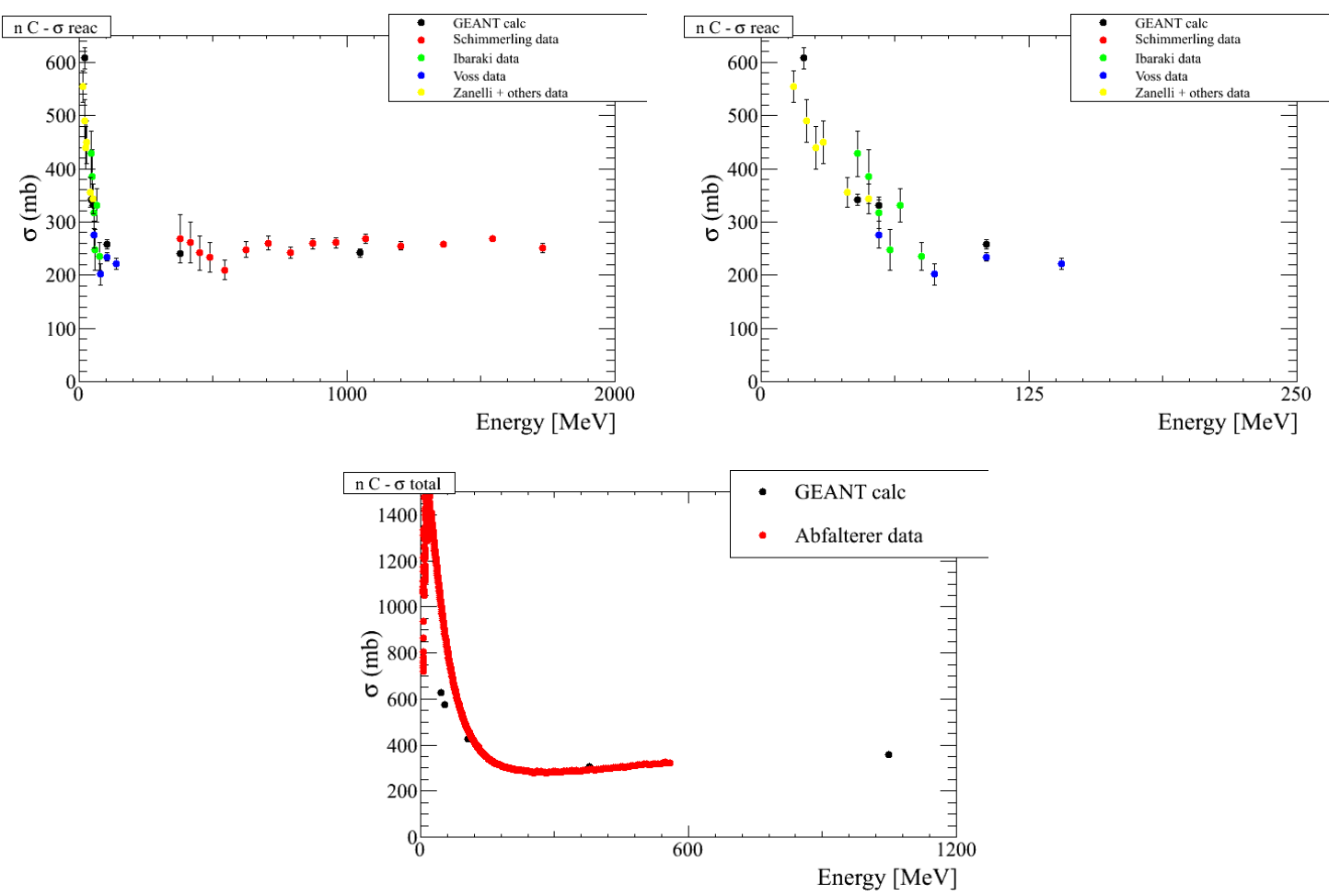

Figure 5.31: Two different ranges of energy are shown for the neutron-carbon inelastic cross-section (labeled $\sigma$ reac) using four different data sets [111, 112, 113, 114] along with a comparison to Geant4 predictions. The total neutron-carbon cross-section for an external data set [115] along with Geant4 comparisons is also shown. We find that while the inelastic cross-section is reasonably modeled, the total cross-section is not. These plots were made by S. Dytman et al. of the MINER $\nu \mathrm{A}$ collaboration.

al. [112], Schimmerling et al. [113], Voss et al. [114], and Abfalterer et al. [115]. The results of those studies for neutron cross-section data on carbon, iron, and lead are shown in Figures 5.31, 5.32, and 5.33. We find good agreement between external data sets and the neutron inelastic cross-section for carbon, iron, and lead. We find noticable discrepancies between the predictions made by Geant 4 and external neutron total cross-section data.

After completion of these studies, we assume an uncertainty on the neutron calorimetric response within the detector of $\pm 20 \%$, which is intended to be conser- 

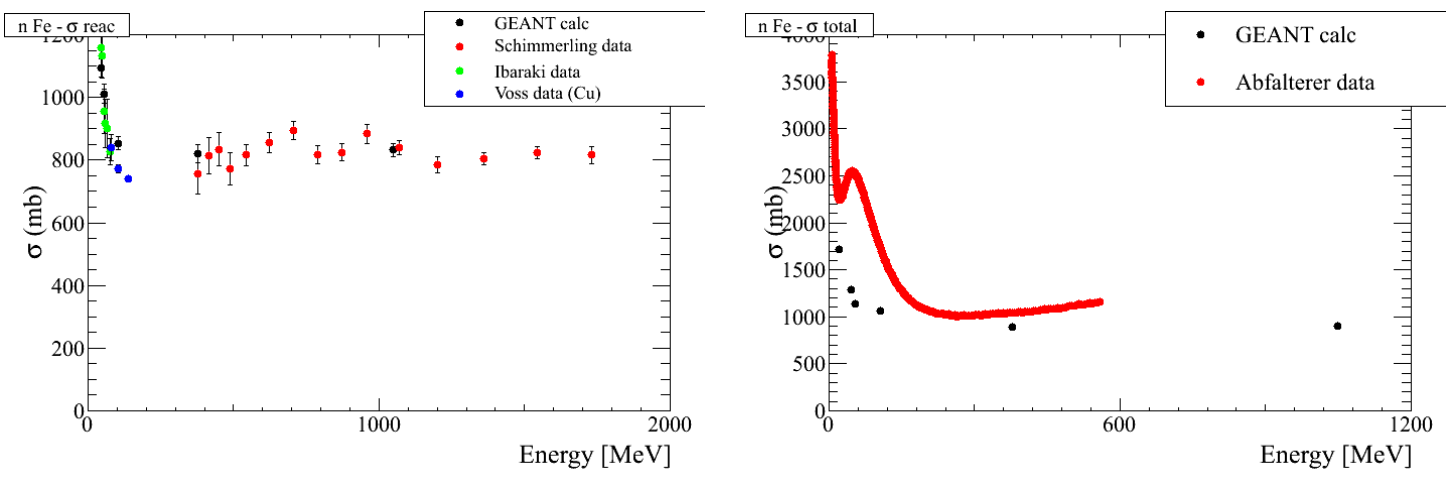

Figure 5.32: Neutron-iron inelastic cross-section (labeled $\sigma$ reac) results for three different data sets $[112,113,114]$ along with Geant4 prediction. The total neutron-iron cross-section for an external data set [115] along with Geant4 comparisons is also shown. These plots were made by S. Dytman et al. of the MINER $\nu \mathrm{A}$ collaboration.
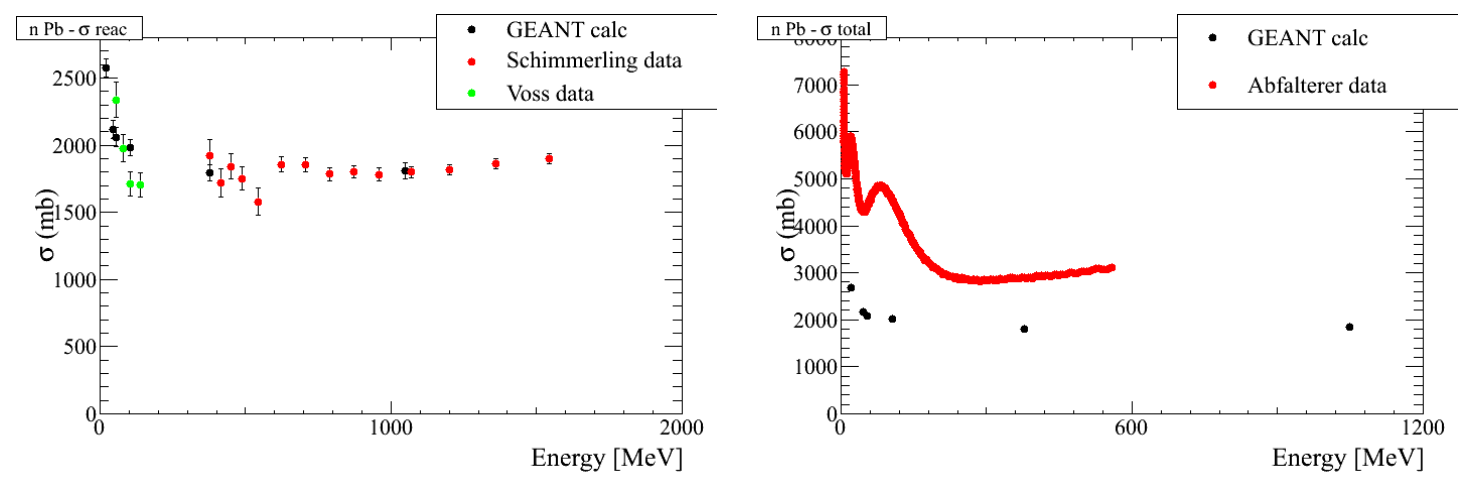

Figure 5.33: Neutron-lead inelastic cross-section (labeled $\sigma$ reac) results for two different data sets $[112,113]$ along with Geant4 predictions. The total neutron-lead cross-section for an external data set [115] along with Geant4 comparisons is also shown. These plots were made by S. Dytman et al. of the MINER $\nu \mathrm{A}$ collaboration. 


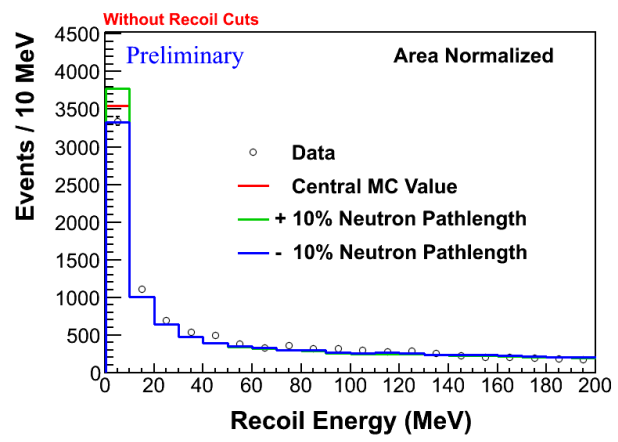

Figure 5.34: A comparison of neutron pathlength in the simulation, samples reweighted to give $\pm 10 \%$ variations of the neutron pathlength, and data. Varying the neutron pathlength in simulation appears to have the greatest effect at low recoil energy.

vative. To assess the systematic error from this, the observed energy deposit caused by a primary neutron interacting in the detector is shifted up or down by $20 \%$.

In, Figure 5.34, a comparison is shown between the default simulation, $\pm 10 \%$ variations of the neutron pathlength in simulation, and data. Most of the impact of this uncertainty can be seen in the case where a low amount of recoil energy is observed within the detector. Most of the disagreement between data and the simulation at low recoil could be explained by uncertainty on the neutron pathlength in the simulation.

For both neutron pathlength and calorimetric response, we repeat background subtraction and efficiency correction steps. The systematic error is then extracted using Equation 5.9.

\section{Proton, Pion, Kaon, Electron, and Photon Response Uncertainties}

We use several methods to address the models uncertainties for predicting proton, pion, electron, and photon energy deposits in the detector, which we refer to as the "response." We use a study similar to the one employed for neutrons to understand 

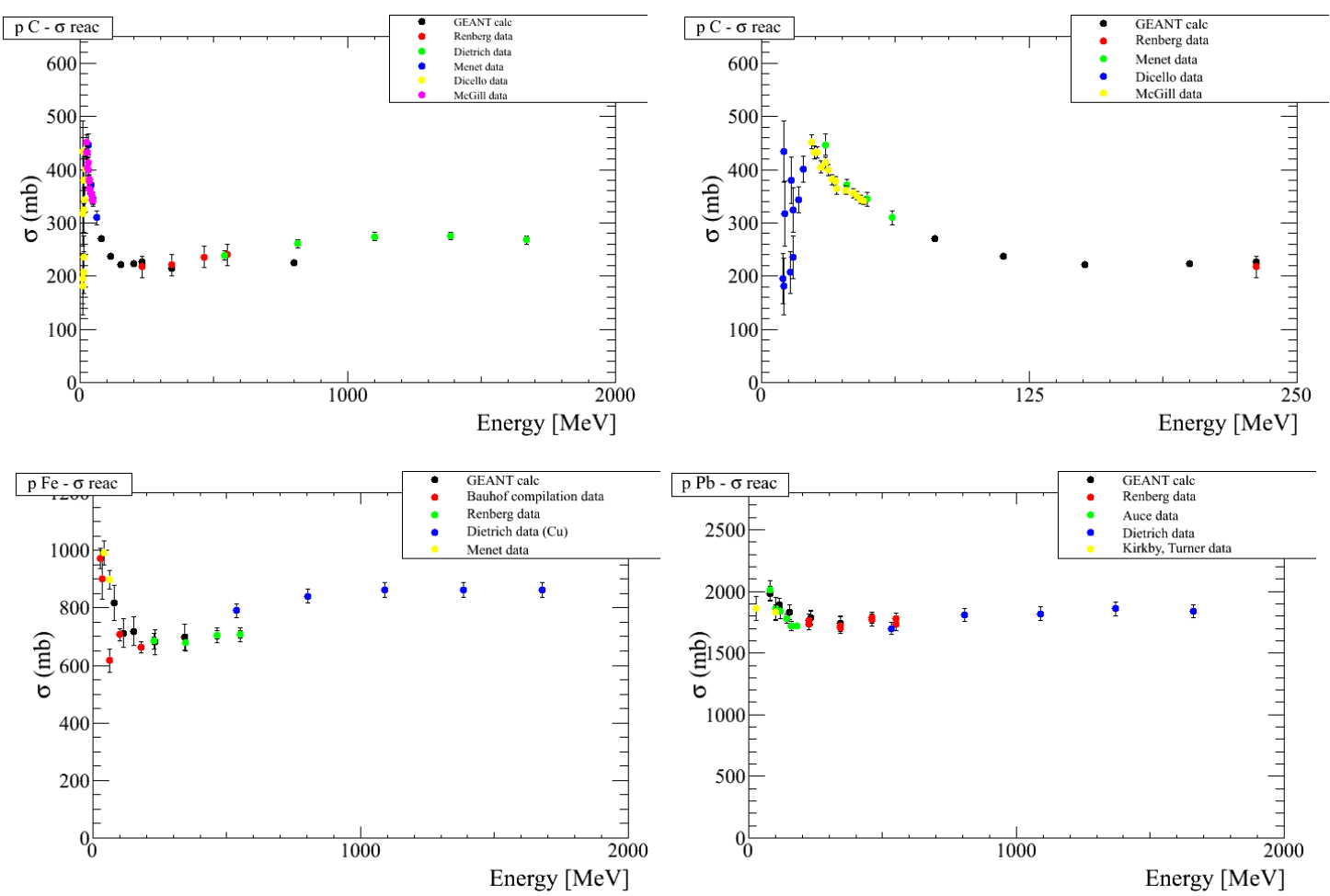

Figure 5.35: Two different ranges of energy for proton-carbon inelastic cross-sections (labeled $\sigma$ reac) as well as proton-iron and proton-lead inelastic cross-sections (labeled $\sigma$ reac) for external data sets $[116,117,118,119,120,122]$ along with Geant4 predictions. These plots were made by S. Dytman et al. of the MINER $\nu \mathrm{A}$ collaboration.

pion and proton repsonse. To apply a systematic error for proton response, the study uses fixed target proton-nucleus scattering data from Dietrich et al. [116], Menet et al. [117], McGill et al. [118], Renberg et al. [119], Dicello et al. [120], Zanelli et al. [111], and MacGregor et al. [121]. To apply a systematic error for pion response, fixed target pion-nucleus scattering data from Bauhof et al. [122], Ashery et al. [123], Wilkin et al. [124], Clough et al. [125], Alderdyce et al. [126], and Gelderloos et al. [127] are used. Comparisons are then made between data and the Geant4 predictions using the same target and beam energy as the data.

Comparisons for proton scattering data for carbon, iron, and lead with Geant4 

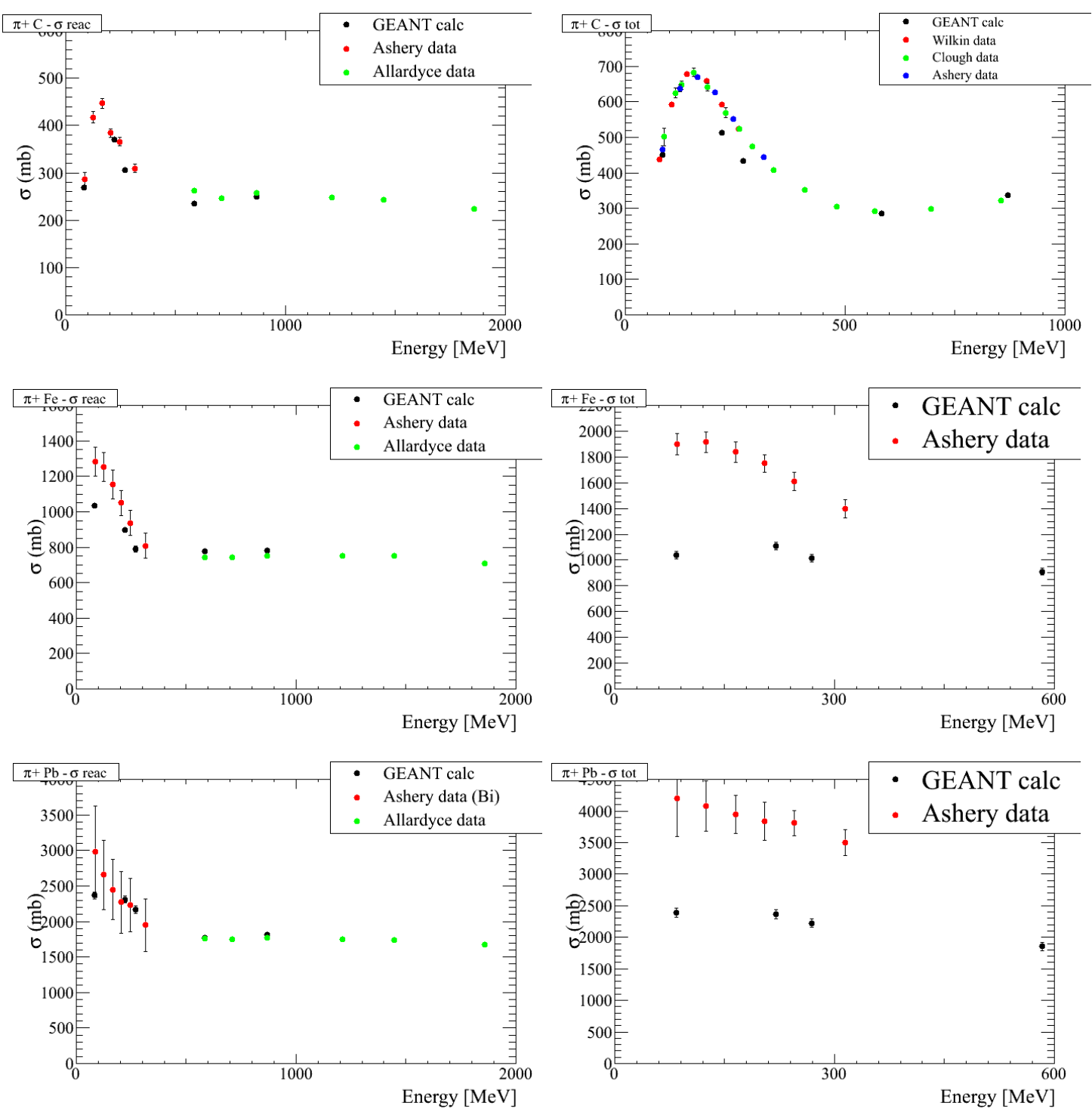

Figure 5.36: $\pi^{+}$inelastic cross-sections (labeled $\sigma$ reac) on carbon, iron, and lead for external data sets $[123,124,125,126]$ along with Geant4 predictions. The total $\pi^{+}$crosssections on carbon, iron, and lead for external data sets [123, 124, 125] along with Geant4 comparisons are also shown. These plots were made by S. Dytman et al. of the MINER $\nu \mathrm{A}$ collaboration. 


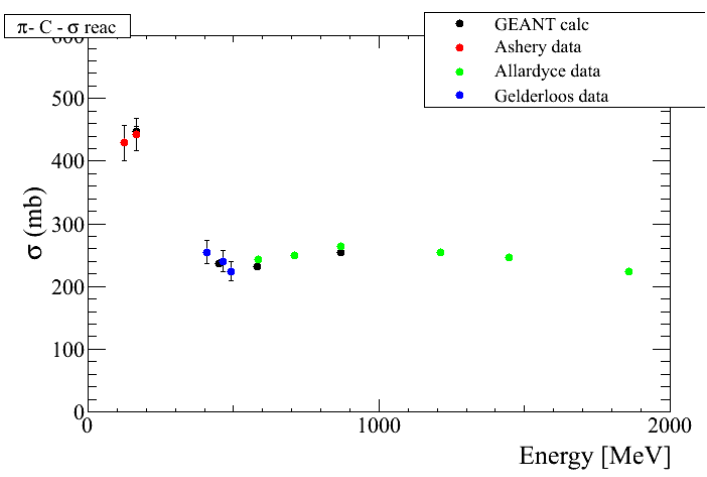

Figure 5.37: $\pi^{-}$-carbon inelastic cross-section (labeled $\sigma$ reac) for external data sets $[123$, 126, 127] along with Geant4 predictions. These plots were made by S. Dytman et al. of the MINER $\nu \mathrm{A}$ collaboration.

are shown in Figure 5.35. We find good agreement between external data and Geant4. We show $\pi^{+}$-nucleus (carbon, iron, and lead) inelastic and total crosssections comparisons between external data and Geant4 in Figure 5.36. The inelastic cross-section on carbon, iron, and lead as well as the total cross-section for $\pi^{+}$carbon scattering show good agreement with Geant4. We see that the total $\pi^{+}$-iron and $\pi^{+}$-lead cross-section has significant disagreement with Geant4. We find good agreement in the comparison of the $\pi^{-}$-carbon inelastic cross-section for external data with Geant4, which is shown in Figure 5.37.

We also consider data from a dedicated test beam detector (T977) involving MINER $\nu \mathrm{A}$ detector components. Data from T977 is used to measure the uncertainty on pion and proton response by probing differences in predicted and observed response for various particles. The method involves selecting particular momenta of particles to strike the detector. The detector is then tuned to the simulation based on the expected response of muons which are also present in the beam. Since muon energy loss is generally well understood, this correctly normalizes the energy response in the test beam detector. Differences between the response in data and 

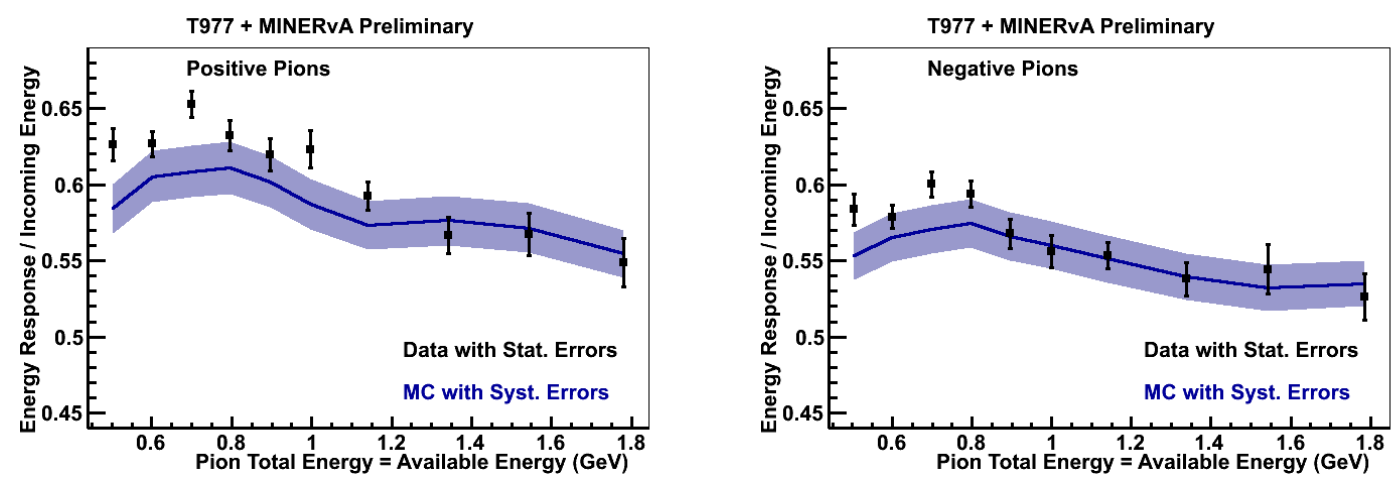

Figure 5.38: Data and simulation overlays showing the energy response to positive and negative pions in the MINER $\nu \mathrm{A}$ test beam detector (T977). These plots were made by R. Gran of the MINER $\nu \mathrm{A}$ collaboration.

simulation for pions and protons are then characterized. Results of this study for $\pi^{+}$ and $\pi^{-}$are shown in Figure 5.38 in which comparisons between data and simulation are made. We consider that disagreement when assessing what systematic error is appropriate. Both this method and data from fixed target experiments mentioned above ensure that uncertainty on pion and proton response is not underestimated.

To find the systematic error for electron response, a comparison is made of Michel electrons from pion decays in data and the simulation. See Figure 5.39 for a comparison of $\frac{\mathrm{dE}}{\mathrm{dx}}$ and reconstruction energy for Michel electrons. Due to the close simularity of how electrons and photons interact in that energy range, this study establishes a systematic error on both the response of photons and electrons.

Kaons response is folded into the response of photons and electrons for the purposes of assessing systematic error. In this case, a systematic error for proton, pion, and together kaon, electron, and photon response of $\pm 10 \%$ is applied. In each case, after shifting response up or down by $10 \%$, we repeat background subtraction and efficiency corrections. The differential cross-section is then remeasured and as before, a systematic error is applied using Equation 5.9. 

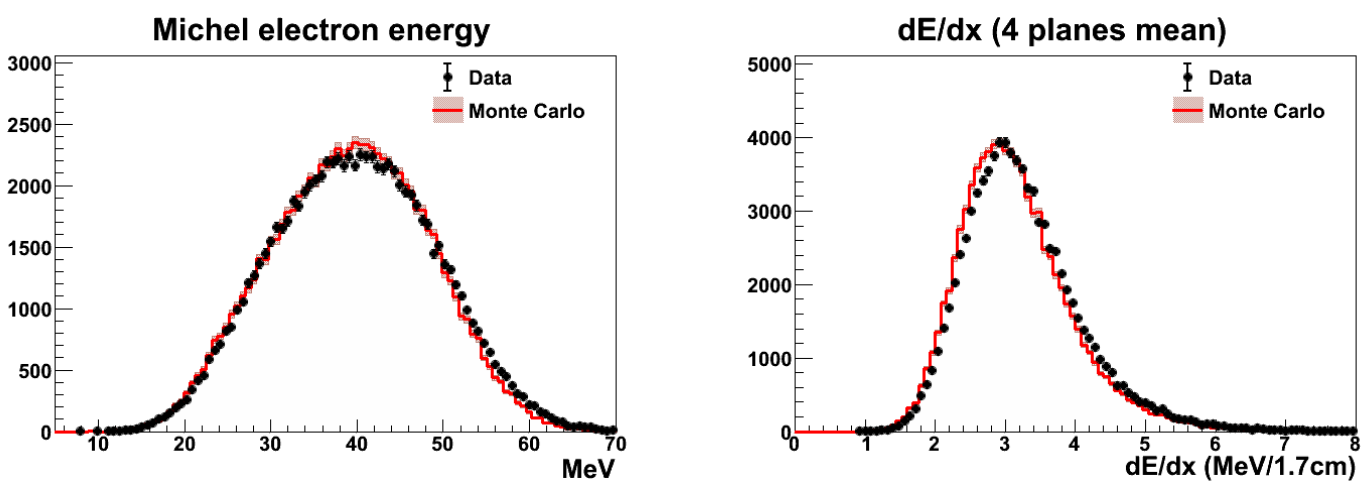

Figure 5.39: Comparisons of energy and $\frac{\mathrm{dE}}{\mathrm{dx}}$ for Michel electrons in data and simulation. Discrepancies between data and simulation are used to extract a systematic error. Plots made by J. Park of the MINER $\nu$ A collaboration.

\subsubsection{Uncertainties on Recoil Energy, Recoil Multiplicity, and Event Selection}

\section{Uncertainty on Recoil Energy and Recoil Multiplicity}

Three cuts involving recoil multiplicity and recoil energy are employed to select the signal sample as is described in Section 5.1.3. To estimate the systematic uncertainty from these selection cuts, several strategies are used.

To apply a systematic error on the signal selection cuts on isolated blob and track multiplicity, first it is noted that data and the simulation agree at about the $20 \%$ level in the distributions in Figure 5.4. An error is then assessed by repeating the analysis after turning one of the cuts off. Background subtraction and efficiency corrections are then repeated. We then find the systematic uncertainty by taking $20 \%$ of the error found using Equation 5.8. This is done for both of the multiplicity cuts.

To estimate the systematic uncertainty from cutting on the observable recoil energy in the detector, a different method is used. Figure 5.5 shows that we see 

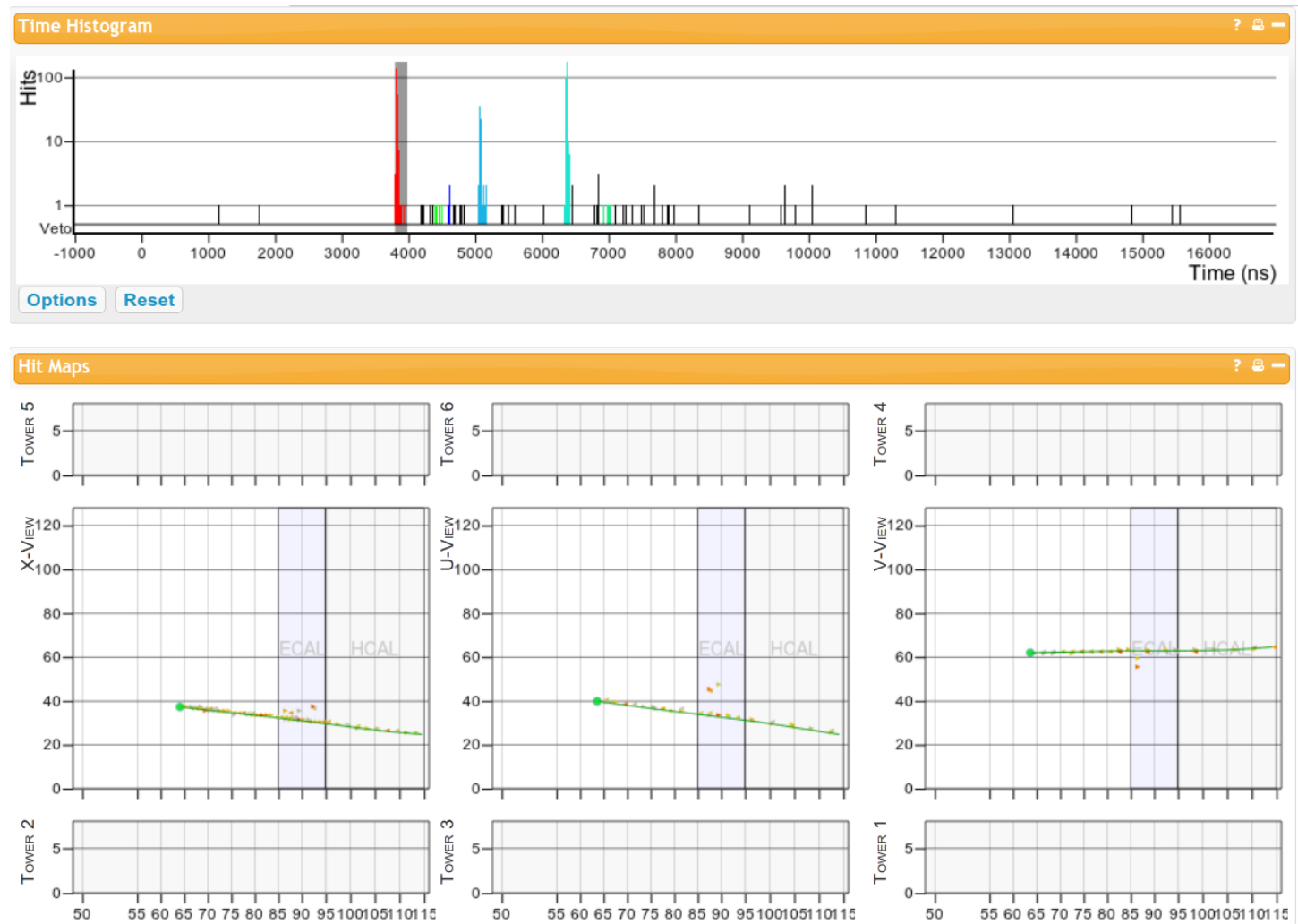

Figure 5.40: Example of a possible bremsstrahlung photon or delta ray from a muon in data. Energy is deposited off of the muon track between modules 86 and 96 in all three views. Activity below roughly $1 \mathrm{MeV}$ is not shown.

fewer events in data than simulation in the lowest recoil energy bin and that we see an excess of events in data between 20 and $100 \mathrm{MeV}$. A hand scan of events at very low recoil energies suggests the simulation may be mis-modeling bremsstrahlung radiation and delta-rays from the muon track. Figure 5.40 shows an example of a muon radiating either a bremsstrahlung photon or delta ray in data. Concern exists on whether the simulation is modeling this correctly.

The method employed to account for this possible modeling error is to take energy deposits in data that are within a cylinder which starts $5 \mathrm{~cm}$ upstream of the track and within a $5 \mathrm{~cm}$ radius along the track with the requirement that the 
deposits are not part of the reconstructed muon. We then overlay the energy from the cylinder on each Monte Carlo event, using a unique data event in each case. A comparison of the recoil energy before and after the overlays is shown in Figure 5.41. Agreement in the lowest recoil energy bin between data and the simulation is very good after the overlay.

The analysis is then repeated with the recoil energy modified by the overlay. Background estimates and efficiency corrections are recalculated and applied. The systematic error is then found using Equation 5.8.
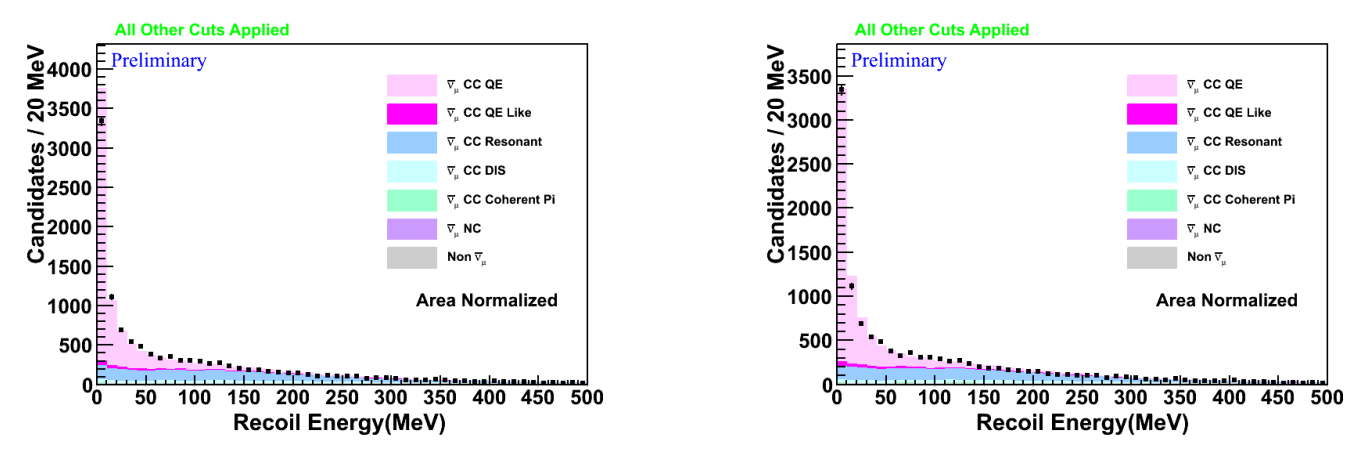

Figure 5.41: On the left is the recoil energy distribution prior to the overlay. On the right is recoil energy after the overlay of energy deposits near the muon track from data. Agreement in the lowest recoil energy bin is improved by the overlay.

\section{Effect of Overlapping Activity in Data}

We restrict our consideration of isolated blobs, extra tracks, and activity that goes into the calculation of recoil energy to only those objects found within a window of \pm 25 ns around the muon track. This is to avoid coincident activity that can add selection inefficiencies. Despite employing this window, we still observe coincident activity that is present in data but not in the simulation. Since we cannot yet add this as part of the simulation, our strategy is to see what the impact is of magnifying 
the effect and using that to find the systematic error. To estimate the effect of not simulating overlapping activity, we widen the time window by $50 \%$. We recalculate uncorrected data distributions and repeat all steps for background subtraction and efficiency corrections. The differential cross-section is found and the systematic error applied using Equation 5.8.

\section{MEU Calibration Uncertainty}

A calibration to tune the energy response in scintillator is performed. This is tuning to a Muon Energy Unit (MEU) and is described in Section 4.1.2. This tuning accounts for slight variations such as in plane thickness and slight errors in the Bethe-Bloch formula. Figures 4.8 and 4.9 are used to find the systematic error for calibration. An overall $2 \%$ error is found along with scaling factors that are dependent upon module. Errors relating to the calibration for variations between strips which we describe in Section 4.1.2 are also folded into this value. We rescale cluster energy by the module dependent scale factor and apply a $2 \%$ smearing to estimate the systematic error on clusters.

We recalculate background estimates and efficiencies and extract the differential cross-section using this sample. The systematic error is then found using Equation 5.8.

\section{Cross-talk Uncertainty}

Cross-talk, also referred to as xtalk in some figures, involves leakage of signal from one channel to another. Two identifiable types of cross-talk are present in MINER $\nu \mathrm{A}, \mathrm{PMT}$ cross-talk and electronics cross-talk. In the PMT, cross-talk can happen if a fiber is not properly aligned over a pixel. This can lead to other pixels 
being illuminated when a given scintillator strip sees a signal. Cross-talk can also occur during the many acceleration stages in the PMT where errant electrons can create signal on neighboring PMT pixels. Cross-talk also occurs within the TriP-t's located within the FEBs described in Section 3.7.1. Various bench tests have been performed to understand at what levels cross-talk occurs in the different stages. The bench tests demonstrate that cross-talk within FEBs to be small and that PMTs are the dominate source of cross-talk.

Results from the above bench tests and other studies are leveraged to make a special cross-talk simulation. For PMT cross-talk, the model gives the number of cross-talk photons assuming the data follow a Poisson distribution. These are photons that strike the wrong PMT dynode. Variations of cross-talk levels between individual channels is also tuned by comparing data and the simulation. The channel by channel variation stems from small misalignments during the PMT assembly. A model is also created for the leakage of current from one PMT dynode to neighboring dynodes, which is also based on bench tests. For electronics cross-talk, the simulation uses direct measurements from bench test data for cross-talk leakage from one channel to another. The resulting charge from cross-talk activity is then propagated through each step of the readout chain and then through the remaining steps of the simulation chain. We use this model as a variation to the nominal simulation. The analysis is repeated with this simulation and background subtraction and efficiency correction are recalculated. The differential cross-section is remeasured and the systematic error applied using Equation 5.8.

The systematic errors found from this cross-talk simulation are sizable, particularly at higher $\mathrm{Q}_{\mathrm{QE}}^{2}$. We find errors are large since cross-talk activity within the simulation leads to a big increase in the number of isolated blobs compared to data 
or the nominal simulation with a disproportionately large effect at high $\mathrm{Q}_{\mathrm{QE}}^{2}$. If we repeat the analysis without cutting on isolated blobs, we find the systematic error from cross-talk shrinks dramatically at the highest values of $\mathrm{Q}_{\mathrm{QE}}^{2}$. Overall, due to the effect on isolated blobs, we find this systematic error is overly conservative and expect improvements to be made in later analyses.

\section{Hot Channels Uncertainty}

We found several channels consistently exhibiting very high activity. Only a handful of the thousands of channels in MINER $\nu \mathrm{A}$ have this issue. These "hot channels" can have activity consistent with energy deposits of 100s or 1000s of MeV. In many cases, the activity is beyond the dynamic range of the electronics suggesting a miscalibration. To apply a systematic error to account for this effect, we study a reconstruction variaton where we veto any hits with more than $100 \mathrm{MeV}$. We then recalculate the background subtraction and efficiency corrections and extract the modified differential cross-section. We then extract a systematic error using Equation 5.8. We find these hot channels introduce very little systematic error.

\subsubsection{Systematic Error on the Flux}

To apply a systematic error on the flux, we use what we refer to as the "1000 Universes" method, which is based on [128]. This method involves sampling an entry randomly from within a Guassian error band based on estimates of one-sigma uncertainties of various parameters, finding a reweighting term for that shifted distribution, and then repeating the analysis under that scenario. This random selection process is repeated 1000 times and the systematic error is then extracted using Equation 5.10. 


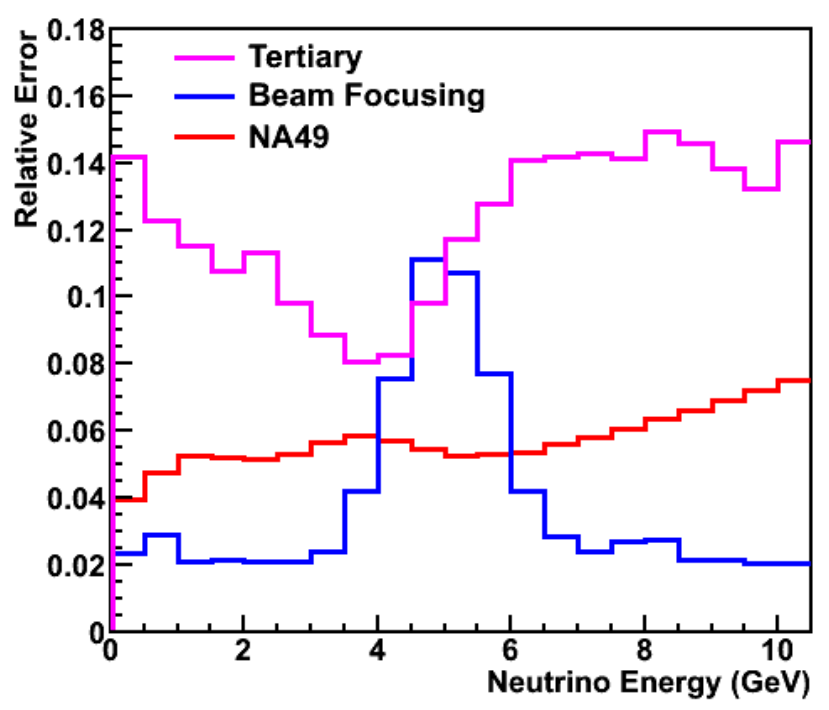

Figure 5.42: The different components of the error on the flux. The components come from pion and kaon focusing, hadronic interactions and reinteractions in the target, and the NA49 reweighting procedure. Plot made by M. Jerkins of the MINER $\nu$ A collaboration.

This method is applied separately for fundamentally different sources of error. One such source is from the modeling of tertiary production of pions and kaons from reinteractions within the target. We refer to this systematic as "Tertiary Flux" error.

We also apply this method to uncertainties that come from the modeling of the focusing of particles in the horn system. We refer to these as "Beam Flux" errors. The horn system is described in Section 2.2. Both Tertiary Flux and Beam Flux errors are extracted by using the one sigma error band on the simulation in finding a "universe."

We also apply this method to errors that come from reweighting using NA49 and Barton data. These data are used in reweighting the flux to more accurately reflect the actual distributions of pions and kaons that are produced off the target. For this last error, direct cross-section measurements are used in the error. We draw 
from values within the one sigma error band of that cross-section measurement in creating the universe that we use to extract the systematic error. We call this "Flux NA49" error. The relative errors for the Tertiary Flux, Beam Flux, and NA49 Flux as a function of neutrino energy are shown in Figure 5.42.

For each class of flux errors, background subtraction, the flux, and efficiencies are recalculated for each of the 1000 variations and the differential cross-section remeasured. In all cases, we use Equation 5.10 to find the systematic error.

\subsubsection{Miscellaneous}

\section{Error on the Number of Target Protons}

There are several other sources of systematic uncertainty that we consider. Uncertainty on the fiducial mass is mentioned in Section 5.2.5 which discusses the number of target protons. This uncertainty comes from the uncertainty on the mass of the different components in the fiducial volume used in this analysis. A list of the error on the relative component of each of these is given in Table 5.10. The total mass of the fiber and scintillator is measured and an uncertainty applied based on those measurements. Additionally, the amount of epoxy and light sealing elements in each module is estimated based on the amount of materials used and the expected volume each occupies. As mentioned in the above section, a systematic uncertainty of $\pm 1.4 \%$ is found. This is applied directly to the differential cross-section result.

\section{Error on the Rock Muon Contamination}

Section 5.2.3 describes the flat efficiency corrections including a correction for those events lost by cuts intended to remove rock muon background. These cuts involve 


\begin{tabular}{c|c|c}
\hline \hline Material & Proportional Amount & Uncertainty \\
\hline Scintillator \& WLS Fiber & $89.47 \%$ & $1.5 \%$ \\
Skin & $5.93 \%$ & $5.0 \%$ \\
Epoxy & $4.20 \%$ & $5.0 \%$ \\
Tape & $0.41 \%$ & $5.0 \%$ \\
\hline Total & 100.01 & $1.39 \%$ \\
\hline \hline
\end{tabular}

Table 5.10: The uncertainty on the masses of the different components that form a plane. Note that total is greater than $100 \%$ due to rounding errors. The uncertainty we apply of $1.5 \%$ is conservative given the above estimates.

cutting on deadtime as well as cutting on upstream activity. Although an efficiency correction is made, some uncertainty exists on whether some of the events removed by the cuts are signal or rock muon background events. To estimate the number of candidates lost due to these cuts, a scan in Arachne is performed. We found 197/250 events are clearly identified as rock muons. Of 53 remaining events, 22 are clearly identified as signal. Figure 5.2 shows an example of a clear rock muon event removed by the veto while Figure 5.3 shows a clear signal event lost due to applying the veto.

The remaining 31 events appear signal-like, but with an upstream time slice close enough that the event could have orignated from a rock muon. Figure 5.43 shows an example of a such an event. We estimate that $36 \pm 16$ signal events are lost due these cuts. This represents a $0.55 \pm 0.25 \%$ efficiency correction. We apply a $\pm 0.25 \%$ systematic uncertainty on the differential cross-section.

\subsubsection{Total Systematic Error}

The uncertainties found for this analysis are the first estimates made of systematic error for MINER $\nu \mathrm{A}$. To avoid understating systematic errors, they are often applied conservatively. Future analyses will have lower systematic errors as model tuning and other studies to more precisely measure the errors are performed. 


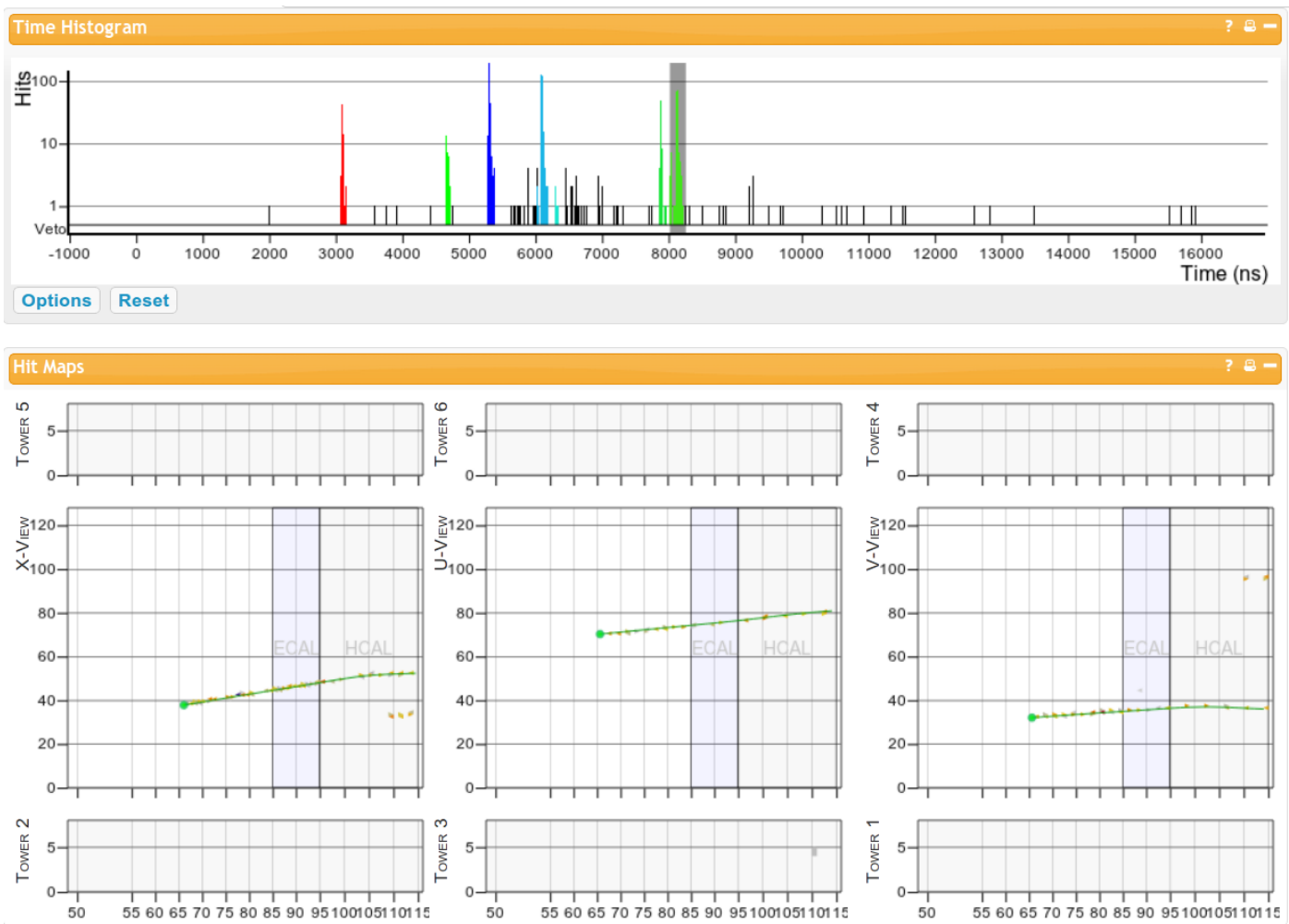

Figure 5.43: An example of an event vetoed by the deadtime cut that could be potentially be signal. Deadtime is present in the event due to a prior time slice, but no visual evidence exists that this is a rock muon. Still, a scenario could exist where all hits prior to module 65 are lost due to deadtime. This and similar events contribute to the $0.25 \%$ systematic error we find the for applying the rock muon veto. Note that activity below roughtly 0.5 $\mathrm{MeV}$ is not shown.

Most of the systematic errors are small. The largest constributions to systematic errors come from muon energy reconstruction, the modeling of neutron response and pathlength, cross-talk, and the flux. Total systematic error is generally very large for the highest bins of $\mathrm{Q}_{\mathrm{QE}}^{2}$. The correlation matrix for $\frac{\mathrm{d} \sigma}{\mathrm{dQ}_{\mathrm{QE}}^{2}}$ can be found in Table 5.11. A full listing of systematic errors by type can be found in Table 5.12. Alternatively, a breakdown of different types of systematic errors for a general types of systematic errors can be found in Figures 5.44 and 5.45 .

We applied the systematic errors found above to the differential cross-section 

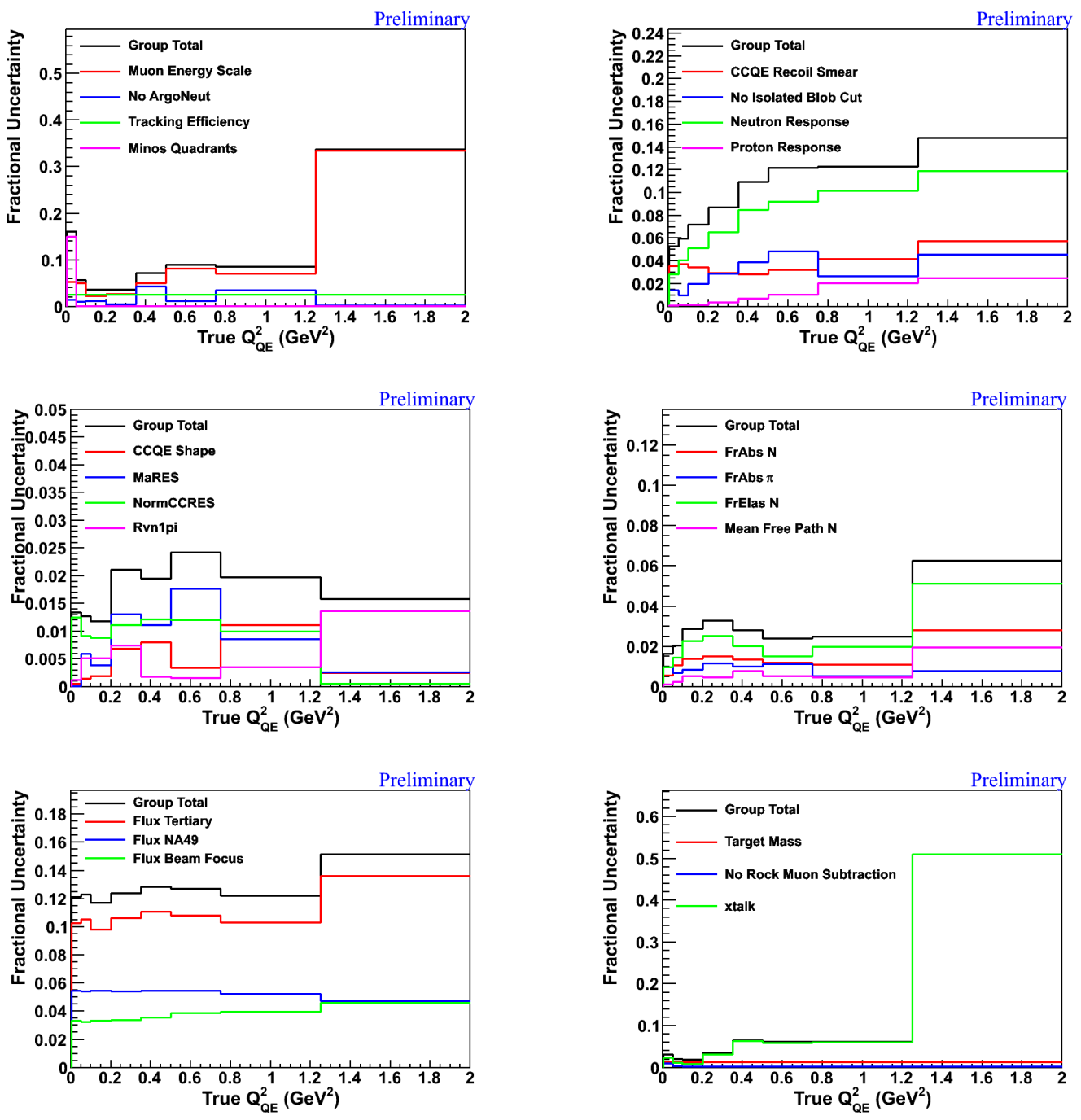

Figure 5.44: Systematic errors by general type.

\begin{tabular}{|c|c|c|c|c|c|c|c|c|c|}
\hline \multirow[b]{2}{*}{$\mathrm{Q}_{\mathrm{QE}}^{2}\left(\mathrm{GeV}^{2}\right)$} & \multicolumn{7}{|c|}{$\mathrm{Q}_{\mathrm{QE}}^{2}\left(\mathrm{GeV}^{2}\right)$} & \multirow[b]{2}{*}{1.25} & \multirow[b]{2}{*}{2.0} \\
\hline & 0.0 & 0.05 & 0.1 & 0.2 & 0.35 & 0.5 & 0.75 & & \\
\hline 0.0 & 1.00 & 0.70 & 0.66 & 0.65 & 0.49 & 0.44 & 0.44 & 0.15 & 0.05 \\
\hline 0.05 & & 1.00 & 0.96 & 0.94 & 0.71 & 0.64 & 0.64 & 0.16 & 0.05 \\
\hline 0.1 & & & 1.00 & 0.98 & 0.84 & 0.78 & 0.75 & 0.25 & 0.17 \\
\hline 0.2 & & & & 1.00 & 0.88 & 0.81 & 0.79 & 0.35 & 0.23 \\
\hline 0.35 & & & & & 1.00 & 0.95 & 0.92 & 0.64 & 0.58 \\
\hline 0.5 & & & & & & 1.00 & 0.89 & 0.69 & 0.62 \\
\hline 0.75 & & & & & & & 1.00 & 0.65 & 0.61 \\
\hline 1.25 & & & & & & & & 1.00 & 0.91 \\
\hline 2.0 & & & & & & & & & 1.00 \\
\hline
\end{tabular}

Table 5.11: The correlation matrix for the systematic errors on the differential crosssection $\frac{\mathrm{d} \sigma}{\mathrm{dQ}_{\mathrm{QE}}^{2}}$ on $\mathrm{CH}$. The $2 \mathrm{GeV}^{2}$ bin is not reported as part of the cross-section result. 


\begin{tabular}{|c|c|c|c|c|c|c|c|c|c|}
\hline Bins & $\operatorname{Bin} 1$ & Bin 2 & Bin 3 & $\operatorname{Bin} 4$ & $\operatorname{Bin} 5$ & $\operatorname{Bin} 6$ & $\operatorname{Bin} 7$ & $\operatorname{Bin} 8$ & Bin 9 \\
\hline \multicolumn{10}{|l|}{ Muon Reconstruction } \\
\hline Muon Energy & 0.052 & 0.050 & 0.023 & 0.026 & -0.050 & -0.082 & -0.071 & -0.335 & -0.449 \\
\hline ArgoNeuT & 0.014 & 0.010 & -0.012 & -0.005 & -0.043 & -0.012 & -0.036 & 0.003 & -0.077 \\
\hline Muon Angle & 0.002 & 0.003 & 0.001 & -0.001 & -0.015 & 0.025 & -0.020 & 0.027 & 0.029 \\
\hline Muon Vertex & 0.002 & 0.000 & 0.000 & 0.001 & 0.001 & 0.001 & -0.000 & -0.000 & 0.002 \\
\hline Minos Tracking Eff & 0.003 & 0.003 & 0.003 & 0.003 & 0.003 & 0.003 & 0.003 & 0.003 & 0.003 \\
\hline Rock Muon Veto & 0.003 & 0.002 & 0.003 & 0.003 & 0.003 & 0.003 & 0.003 & 0.003 & 0.003 \\
\hline MINER $\nu \mathrm{A}$ Tracking Eff & 0.025 & 0.025 & 0.025 & 0.025 & 0.025 & 0.025 & 0.025 & 0.025 & 0.025 \\
\hline Minos Acceptance & 0.150 & 0.000 & 0.000 & 0.000 & 0.000 & 0.000 & 0.000 & 0.000 & 0.000 \\
\hline Group Total & 0.162 & 0.057 & 0.036 & 0.037 & 0.072 & 0.090 & 0.086 & 0.337 & 0.458 \\
\hline \multicolumn{10}{|l|}{ Recoil Reconstruction } \\
\hline CCQE Recoil Error & 0.036 & 0.037 & 0.035 & 0.029 & 0.028 & 0.033 & 0.042 & 0.058 & 0.075 \\
\hline Extra Track Cut & 0.003 & 0.003 & 0.002 & 0.005 & 0.005 & 0.010 & 0.013 & 0.017 & 0.010 \\
\hline Isolated Blob Cut & 0.014 & 0.010 & 0.020 & 0.029 & 0.039 & 0.049 & 0.027 & 0.046 & -0.082 \\
\hline Neutron Pathlength & 0.019 & 0.017 & 0.030 & 0.037 & 0.046 & 0.046 & 0.034 & 0.026 & 0.025 \\
\hline Neutron Response & 0.029 & 0.041 & 0.051 & 0.065 & 0.085 & 0.092 & 0.102 & 0.119 & 0.141 \\
\hline Proton Response & 0.001 & 0.001 & 0.002 & 0.004 & 0.007 & 0.011 & 0.020 & 0.025 & 0.030 \\
\hline Pion Response & 0.008 & 0.007 & 0.008 & 0.012 & 0.014 & 0.018 & 0.019 & 0.022 & 0.022 \\
\hline Other Response & 0.006 & 0.007 & 0.007 & 0.007 & 0.008 & 0.010 & 0.012 & 0.015 & 0.017 \\
\hline MEU & 0.001 & 0.001 & 0.001 & 0.002 & 0.001 & 0.003 & -0.002 & -0.002 & -0.008 \\
\hline Hot Channels & 0.000 & -0.002 & 0.000 & -0.004 & 0.001 & -0.001 & 0.001 & 0.002 & 0.009 \\
\hline Recoil Time Window & 0.002 & 0.001 & 0.002 & 0.004 & 0.009 & 0.010 & 0.007 & 0.010 & 0.014 \\
\hline Group Total & 0.053 & 0.060 & 0.072 & 0.087 & 0.110 & 0.122 & 0.123 & 0.148 & 0.187 \\
\hline \multicolumn{10}{|l|}{$\begin{array}{c}\text { Primary } \\
\text { Interaction Models }\end{array}$} \\
\hline CCQE Shape & 0.001 & -0.001 & -0.002 & -0.007 & -0.008 & -0.003 & -0.011 & -0.003 & -0.192 \\
\hline $\mathrm{E}_{\nu}$ Scaled Backgrounds & 0.004 & 0.003 & 0.004 & 0.004 & 0.003 & 0.005 & 0.000 & -0.000 & 0.006 \\
\hline EtaNCEL & 0.000 & 0.000 & 0.000 & 0.000 & 0.000 & 0.000 & 0.000 & 0.000 & 0.000 \\
\hline MaNCEL & 0.000 & 0.000 & 0.000 & 0.000 & 0.000 & 0.000 & 0.000 & 0.000 & 0.000 \\
\hline MaRES & 0.000 & 0.006 & 0.004 & 0.013 & 0.011 & 0.018 & 0.009 & -0.003 & -0.021 \\
\hline MvRES & 0.000 & -0.001 & -0.000 & -0.005 & -0.003 & -0.009 & -0.004 & -0.003 & 0.010 \\
\hline NormCCRES & 0.013 & 0.009 & 0.009 & 0.011 & 0.012 & 0.012 & 0.010 & 0.001 & -0.008 \\
\hline Rvn1pi & 0.001 & 0.005 & 0.005 & 0.007 & 0.002 & 0.002 & -0.003 & -0.014 & -0.032 \\
\hline Rvn2pi & 0.001 & 0.000 & 0.001 & 0.001 & 0.003 & 0.003 & 0.004 & 0.002 & 0.008 \\
\hline Rvp1pi & 0.001 & 0.002 & 0.001 & 0.002 & 0.003 & 0.002 & 0.003 & 0.003 & 0.001 \\
\hline Rvp2pi & 0.001 & 0.000 & 0.001 & 0.002 & 0.003 & 0.003 & 0.006 & 0.005 & 0.013 \\
\hline Group Total & 0.013 & 0.013 & 0.012 & 0.021 & 0.019 & 0.024 & 0.020 & 0.016 & 0.196 \\
\hline \multicolumn{10}{|l|}{$\begin{array}{c}\text { Final State } \\
\text { Interaction Models }\end{array}$} \\
\hline AGKYxF1pi & 0.000 & -0.000 & 0.000 & 0.001 & 0.001 & 0.001 & 0.001 & 0.004 & 0.004 \\
\hline FrAbs $\mathrm{N}$ & 0.006 & 0.011 & 0.014 & 0.015 & 0.014 & 0.012 & -0.011 & -0.028 & -0.054 \\
\hline $\operatorname{FrAbs} \pi$ & 0.010 & 0.007 & 0.008 & 0.012 & 0.010 & 0.011 & 0.005 & 0.008 & 0.001 \\
\hline FrCEx $N$ & 0.000 & -0.000 & 0.001 & -0.000 & -0.000 & -0.001 & -0.001 & -0.002 & -0.001 \\
\hline FrCEx $\pi$ & 0.003 & 0.002 & 0.002 & 0.003 & 0.004 & 0.004 & 0.003 & 0.002 & -0.002 \\
\hline FrElas N & 0.010 & 0.015 & 0.023 & 0.025 & 0.020 & 0.015 & -0.020 & -0.051 & -0.080 \\
\hline FrElas $\pi$ & 0.004 & 0.003 & 0.003 & 0.004 & 0.004 & 0.004 & 0.003 & 0.002 & 0.004 \\
\hline FrInel N & 0.000 & -0.000 & -0.000 & 0.000 & -0.001 & -0.000 & 0.001 & 0.001 & 0.002 \\
\hline FrInel $\pi$ & 0.001 & -0.001 & 0.002 & 0.003 & -0.001 & 0.001 & -0.003 & 0.003 & 0.001 \\
\hline FrPiProd N & 0.000 & 0.000 & 0.000 & 0.000 & 0.000 & 0.001 & 0.002 & 0.005 & 0.003 \\
\hline FrPiProd $\pi$ & 0.000 & 0.000 & 0.000 & 0.000 & 0.000 & 0.000 & 0.000 & 0.000 & 0.001 \\
\hline Mean Free Path N & 0.001 & 0.002 & 0.005 & 0.005 & 0.008 & 0.005 & -0.005 & -0.020 & -0.043 \\
\hline Mean Free Path $\pi$ & 0.004 & 0.004 & 0.003 & 0.003 & 0.003 & 0.004 & 0.004 & 0.003 & 0.005 \\
\hline RDecBR1gamma & 0.000 & 0.000 & -0.000 & -0.000 & 0.000 & 0.000 & -0.000 & -0.000 & -0.000 \\
\hline Theta Delta $2 \mathrm{~N} \pi$ & 0.000 & 0.000 & -0.000 & 0.000 & 0.000 & 0.000 & 0.000 & 0.000 & -0.001 \\
\hline Group Total & 0.016 & 0.020 & 0.029 & 0.033 & 0.028 & 0.024 & 0.025 & 0.063 & 0.106 \\
\hline \multicolumn{10}{|l|}{ Flux } \\
\hline Flux Tertiary & 0.103 & 0.106 & 0.098 & 0.106 & 0.111 & 0.108 & 0.103 & 0.136 & 0.116 \\
\hline Flux NA49 & 0.055 & 0.054 & 0.055 & 0.054 & 0.055 & 0.054 & 0.052 & 0.047 & 0.053 \\
\hline Flux Beam Focus & 0.033 & 0.033 & 0.034 & 0.034 & 0.036 & 0.039 & 0.040 & 0.046 & 0.032 \\
\hline Group Total & 0.121 & 0.123 & 0.117 & 0.124 & 0.129 & 0.127 & 0.122 & 0.151 & 0.132 \\
\hline \multicolumn{10}{|l|}{ Other } \\
\hline Target Mass & 0.014 & 0.014 & 0.014 & 0.014 & 0.014 & 0.014 & 0.014 & 0.014 & 0.014 \\
\hline Rock Muon Subtraction & 0.010 & 0.004 & 0.003 & 0.002 & 0.002 & 0.002 & 0.002 & 0.003 & 0.003 \\
\hline Eff Loss from Deadtime & 0.010 & 0.010 & 0.010 & 0.010 & 0.010 & 0.010 & 0.010 & 0.010 & 0.010 \\
\hline xtalk & 0.024 & 0.012 & 0.009 & 0.032 & 0.064 & 0.059 & 0.060 & 0.509 & 0.416 \\
\hline Group Total & 0.031 & 0.021 & 0.020 & 0.036 & 0.066 & 0.061 & 0.062 & 0.510 & 0.416 \\
\hline Total Systematic Error & 0.212 & 0.152 & 0.147 & 0.165 & 0.198 & 0.210 & 0.206 & 0.650 & 0.696 \\
\hline Statistical Error & 0.060 & 0.073 & 0.060 & 0.078 & 0.133 & 0.201 & 0.266 & 0.571 & 1.510 \\
\hline
\end{tabular}

Table 5.12: Fractional systematic uncertainties on $\frac{\mathrm{d} \sigma}{\mathrm{dQ}_{\mathrm{QE}}^{2}}$. Bins 1-9 correspond to the following $\mathrm{Q}_{\mathrm{QE}}^{2}$ bins in units of $\mathrm{GeV}^{2},[0,0.05),[0.05,0.1),[0.1,0.2),[0.2,0.35),[0.35,0.5)$, $[0.5,0.75),[0.75,1.25),[1.25,2.0),[2.0, \infty)$, respectively. 


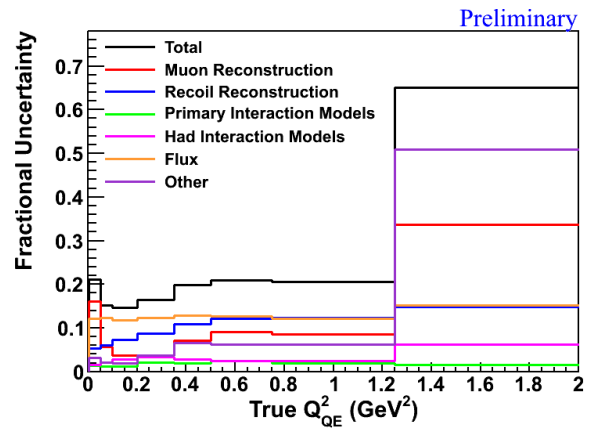

Figure 5.45: Summary of systematic errors.

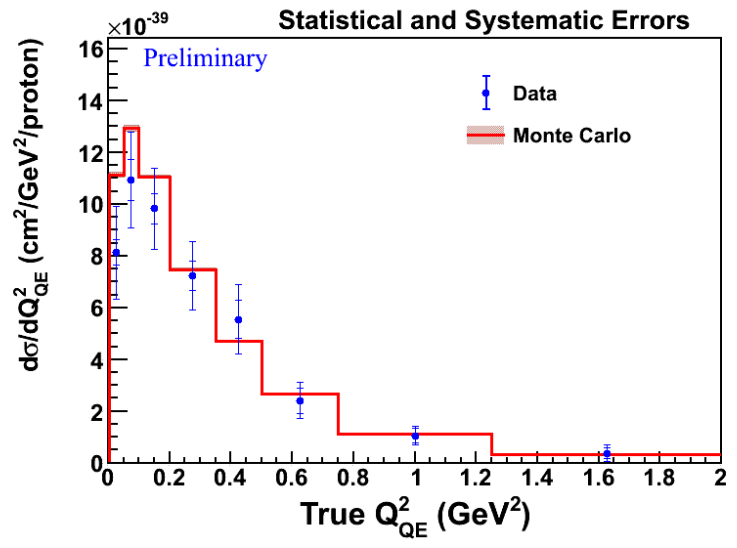

Figure 5.46: The differential cross-section $\frac{\mathrm{d} \sigma}{\mathrm{dQ}_{\mathrm{QE}}^{2}}$ extracted on $\mathrm{CH}$ in MINER $\nu \mathrm{A}$ with statistical and systematic errors applied. The inner error bars are the original statistical errors as found on Figure 5.24. The outer errror bars are the sum in quadrature of the statistical and systematic errors.

$\frac{\mathrm{d} \sigma}{\mathrm{dQ}_{\mathrm{QE}}^{2}}$ shown in Figure 5.46. This measurement is consistent with the value predicted by simulation given the size of the statistical and systematic uncertainties. Further comparisons to other models are made in Section 5.4. 


\subsection{Comparing $\frac{\mathrm{d} \sigma}{\mathrm{dQ}_{\mathrm{QE}}^{2}}$ with Other Models}

We make comparisons of $\frac{\mathrm{d} \sigma}{\mathrm{dQ}_{\mathrm{QE}}^{2}}$ on $\mathrm{CH}$ in MINER $\nu \mathrm{A}$ to predictions provided to us by J. Sobczyk using the Wroclaw Neutrino Event Generator (NuWro) [129]. The GENIE simulation at the time of this analysis does not model MEC or use spectral functions to model nucleons within the nucleus, both of which we discuss in Section 1.3.6. The NuWro generator does employ these elements. We made comparisons to four configurations of the NuWro simulation.

- NuWro RFG Model, $\mathrm{M}_{\mathrm{A}}=0.99 \mathrm{GeV}$, no MEC modeling

- NuWro RFG Model, $\mathrm{M}_{\mathrm{A}}=1.35 \mathrm{GeV}$, no MEC modeling

- NuWro RFG Model, $\mathrm{M}_{\mathrm{A}}=0.99 \mathrm{GeV}$, MEC modeling

- NuWro Spectral Functions, MEC modeling

The first case is very similar to what is implemented in the version of the GENIE simulation used in this analysis including use of the RFG Model. The second case is a similar model but with the axial mass parameter $\mathrm{M}_{\mathrm{A}}$, described in Sections 1.3.5 and 4.2 .3 , set to $1.35 \mathrm{GeV}$. The selection of this value is motivated by results from the MiniBooNE experiment, which extracted that value along with a Pauli Blocking term, $\kappa=1.007$ [46]. This value of $\mathrm{M}_{\mathrm{A}}$ is higher than what many other experiments have historically measured [45] and is required to explain a total CCQE cross-section larger than predicted by models with $\mathrm{M}_{\mathrm{A}}=0.99 \mathrm{GeV}$. The third case involves similar model parameters used to predict the CCQE cross-section in GENIE, but with MEC implemented in the simulation. The last case involves predictions made using spectral functions to describe the distribution of nucleons in the nucleus instead of the RFG Model along with MEC type interactions. 


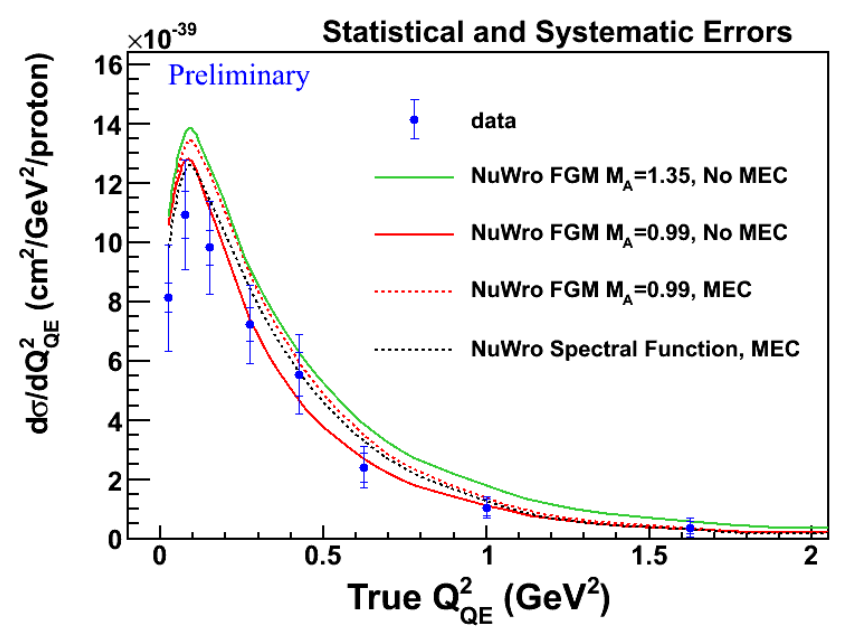

Figure 5.47: A comparison of the CCQE differential cross-section extracted in MINER $\nu \mathrm{A}$ on $\mathrm{CH}$ with various predictions made using the NuWro neutrino simulation. The inner error bars on data points are statistical errors while the outer error bars include both statistical and systematic errors.

An overlay of the different predictions made using NuWro along with the differential cross-section $\frac{\mathrm{d} \sigma}{\mathrm{d} \mathrm{Q}_{\mathrm{QE}}^{2}}$ found on $\mathrm{CH}$ in MINER $\nu \mathrm{A}$ is show in Figure 5.47. Data in this distribution includes both statistical and systematic errors.

We find the differential cross-section $\frac{\mathrm{d} \sigma}{\mathrm{d} \mathrm{Q}_{\mathrm{QE}}^{2}}$ we measure on $\mathrm{CH}$ is consistent predictions made using a value of $\mathrm{M}_{\mathrm{A}}$ of $0.99 \mathrm{GeV}$. Statistical and systematic errors are currently too large to distiguish between the different models. A future iteration of this analysis will benefit from incorporating more data along with reduced systematic errors allowing for more distinguishing power. 


\section{Chapter 6}

\section{Conclusions}

We have presented the first cross-section measurement made using data taken with

the MINER $\nu$ A detector. We have made comparisons of our result $\frac{\mathrm{d} \sigma}{\mathrm{d} \mathrm{Q}^{2}}$ for muon anti-neutrino CCQE scattering on $\mathrm{CH}$ with models contained within the GENIE and NuWro simulations. In making these comparisons, we have examined the agreement of our data with two different values of the axial mass, $\mathrm{M}_{\mathrm{A}}$, both using the RFG model. We have also made comparisons of our result with models involving MEC as well as spectral functions. We find that our data agree with the default model in the GENIE simulation, which employs the RFG model, a value of $\mathrm{M}_{\mathrm{A}}$ of $0.99 \mathrm{GeV}$, and no modeling of MEC. Of the models we examined, we cannot exclude models or ascertain which model is prefered by these data at this time.

Advances in understanding of our systematic error as well as dedicated studies to better understand the flux are underway. More data will be included in analyzing this channel, which necessarily must be done in a separate fashion than the Frozen Detector data. The Frozen Detector data required its own analysis due to the unique fiducial volume of the Frozen Detector as well as the presence of ArgoNeuT. 
We expect a reduction in both our statistical and systematic uncertainty in the next iteration of this analysis. Other elements will be added in future updates to this analysis, specifically, examining energy near the vertex of the interaction. Such information is useful for testing final state interaction models.

Analyses will also be performed for the various nuclear targets present in the full MINER $\nu$ A detector. This includes helium, carbon (graphite), iron, lead, and water targets. A proposal exists to perform tests on a deterium target as well. These targets allow measurements of the cross-section as a function of the atomic mass to be made. Such data will further aid in understanding nuclear effects in neutrino and anti-neutrino interactions. The next analyses of this channel will help improve simulation models for neutrino interactions as well as assist in lowering systematic errors in neutrino oscillation experiments. 


\section{Bibliography}

[1] F. Halzen and D. Martin, "Quarks and Leptons: An Introductory Course in Modern Particle Physics," John Wiley and Sons, Inc. (1984).

[2] J. D. Bjorken and S. D. Drell, "Relativistic Quantum Mechanics," McGrawHill, Inc. (1964).

[3] G. Aad et al. [ATLAS Collaboration], "Observation of a new particle in the search for the Standard Model Higgs boson with the ATLAS detector at the LHC," Phys. Lett. B 716, 1 (2012) [arXiv:1207.7214 [hep-ex]].

[4] S. Chatrchyan et al. [CMS Collaboration], "Observation of a new boson at a mass of $125 \mathrm{GeV}$ with the CMS experiment at the LHC," Phys. Lett. B 716, 30 (2012) [arXiv:1207.7235 [hep-ex]].

[5] J. Beringer et al. (Particle Data Group), Phys. Rev. D86, 010001 (2012).

[6] D. H. Perkins, "Introduction to High Energy Physics," Cambridge University Press, (2000).

[7] B. Kayser, "Neutrino Mass, Mixing, and Flavor Change," (2008). [arXiv:hep$\mathrm{ph} / 08041497]$

[8] G. Arnison, et al. [UA1 Collaboration, CERN, Geneva, Switzerland], "Experimental observation of isolated large transverse energy electrons with associated missing energy at $\mathrm{s}=540 \mathrm{GeV}$," Physics Letters B, Volume 122, Issue 1, 24 February 1983, Pages 103-116, ISSN 0370-2693, 10.1016/0370-2693(83)911772 .

[9] G. Arnison, et al. [UA1 Collaboration,, CERN, Geneva, Switzerland], "Experimental observation of lepton pairs of invariant mass around $95 \mathrm{GeV} / c^{2}$ at the CERN SPS collider," Physics Letters B, Volume 126, Issue 5, 7 July 1983, Pages 398-410, ISSN 0370-2693, 10.1016/0370-2693(83)90188-0. 
[10] G. Bertone, D. Hooper and J. Silk, "Particle dark matter: Evidence, candidates and constraints," Phys. Rept. 405, 279 (2005) [hep-ph/0404175].

[11] A. G. Riess et al. [Supernova Search Team Collaboration], "Observational evidence from supernovae for an accelerating universe and a cosmological constant," Astron. J. 116, 1009 (1998) [astro-ph/9805201].

[12] A. Aguilar et al. (LSND), "Evidence for Neutrino Oscillations from the Observation of Electron Anti-neutrinos in a Muon Anti-Neutrino Beam," Phys.Rev.D64:112007, (2001).

[13] A. A. Aguilar-Arevalo et al. [MiniBooNE Collaboration], "A Combined $\nu_{\mu} \rightarrow \nu_{e}$ and $\bar{\nu}_{\mu} \rightarrow \bar{\nu}_{e}$ Oscillation Analysis of the MiniBooNE Excesses," arXiv:1207.4809 [hep-ex].

[14] G. Mangano, G. Miele, S. Pastor, O. Pisanti and S. Sarikas, "Constraining the cosmic radiation density due to lepton number with Big Bang Nucleosynthesis," JCAP 1103, 035 (2011) [arXiv:1011.0916 [astro-ph.CO]].

[15] J. Schechter and J.W. F. Valle, "Neutrino masses in $\mathrm{SU}(2) \otimes \mathrm{U}(1)$ theories,"Phys. Rev. D22, 2227 (1980).

[16] J. W. F. Valle, "Current theoretical status of neutrino masses and mixing," AIP Conf. Proc. 1382, 9 (2011), DOI:10.1063/1.3644260.

[17] D.W. Schmitz, "A Measurement of Hadron Production Cross-Sections for the Simulation of Accelerator Neutrino Beams and a Search for $\nu_{\mu} \rightarrow \nu_{e}$ Oscillations in the $\Delta \mathrm{m}^{2} \approx 1 \mathrm{eV}^{2}$ Region," PhD Thesis, Columbia University, 2008.

[18] B. T. Cleveland, et al., "Measurement of the solar electron neutrino flux with the Homestake chlorine detector," Astrophysical Journal 496: 505526, (1998).

[19] Y. Fukuda, et al. [The K2K collaboration], "Evidence for oscillation of atmospheric neutrinos," Physical Review Letters 81 (8): 1562-1567, (1998).

[20] M. H. Ahn et al., "Measurement of Neutrino Oscillation by the K2K Experiment," Phys. Rev. D 74, 072003 (2006), [arxiv:hep-ex/0606032].

[21] P. Adamson et al. [MINOS Collaboration], "An improved measurement of muon antineutrino disappearance in MINOS," Phys. Rev. Lett. 108, 191801 (2012) [arXiv:1202.2772 [hep-ex]].

[22] Q. R. Ahmad et al. [SNO Collaboration], "Measurement of the rate of nu/e + $\mathrm{d} \rightarrow \mathrm{p}+\mathrm{p}+e^{-}$interactions produced by B-8 solar neutrinos at the Sudbury Neutrino Observatory," Phys. Rev. Lett. 87, 071301 (2001) [nucl-ex/0106015]. 
[23] K. Eguchi, et al., [KamLAND Collaboration], "First results from KamLAND: evidence for reactor antineutrino disappearance". Physical Review Letters 90 (2): 021802021807, (2003).

[24] F. P. An et al. [DAYA-BAY Collaboration], "Observation of electronantineutrino disappearance at Daya Bay," Phys. Rev. Lett. 108, 171803 (2012) [arXiv:1203.1669 [hep-ex]].

[25] J. K. Ahn, et al. [RENO Collaboration], "Observation of Reactor Electron Antineutrino Disappearance in the RENO Experiment," Physical Review Letters 108 (18): 191802, (2012)

[26] M. Auger et al. [EXO Collaboration], "Search for Neutrinoless DoubleBeta Decay in ${ }^{136}$ Xe with EXO-200," Phys. Rev. Lett. 109, 032505 (2012) [arXiv:1205.5608 [hep-ex]].

[27] J. Argyriades et al., "Measurement of the double-beta decay half-life of ${ }^{150} \mathrm{Nd}$ and search for neutrinoless decay modes with the NEMO-3 detector," Phys. Rev. C 80, 032501(R) (2009).

[28] D. G. Phillips, II, E. Aguayo, F. T. Avignone, III, H. O. Back, A. S. Barabash, M. Bergevin, F. E. Bertrand and M. Boswell et al., "The Majorana experiment: an ultra-low background search for neutrinoless double-beta decay," J. Phys. Conf. Ser. 381, 012044 (2012) [arXiv:1111.5578 [nucl-ex]].

[29] F. Fraenkle, (2011), "KATRIN: an experiment to determine the neutrino mass," Proceedings of the DPF-2011 Conference, arXiv:1110.0087.

[30] K. Abe, et al. [The T2K Collaboration], The T2K experiment, Nuclear Instruments and Methods in Physics Research Section A659, 106-135 (2011).

[31] D. S. Ayres et al. [NOvA Collaboration], "NOvA: Proposal to build a 30 kiloton off-axis detector to study nu(mu) $\rightarrow$ nu(e) oscillations in the NuMI beamline," hep-ex/0503053.

[32] OPERA Collaboration, R. Acquafredda et al., "The OPERA experiment in the CERN to Gran Sasso neutrino beam," JINST 4 (2009) P04018.

[33] D. Griffith, "Introduction to Elementary Particles," John Wiley and Sons, Inc., (1987).

[34] C. H. Llewellyn Smith, "Neutrino Reactions At Accelerator Energies," Phys. Rept. 3, 261 (1972). 
[35] D.H. Wilkinson, "Limits to second-class nucleonic currents," Nuclear Instruments and Methods A455, 656659 (2000).

[36] M. Day and K. S. McFarland, "Differences in Quasi-Elastic Cross-Sections of Muon and Electron Neutrinos," arXiv:1206.6745 [hep-ph].

[37] H. S. Budd, A. Bodek and J. Arrington, "Modeling quasielastic form-factors for electron and neutrino scattering," arXiv:1206.6745hep-ex/0308005.

[38] J.J. Kelly, "Simple parametrization of nucleon form factors," Phys. Rev. C70, (2004).

[39] A. Bodek, S. Avvakumov, R. Bradford and H. S. Budd, "Vector and Axial Nucleon Form Factors:A Duality Constrained Parameterization," Eur. Phys. J. C 53, 349 (2008) [arXiv:0708.1946 [hep-ex]].

[40] M. L. Goldberger and S. B.Treiman, "Decay of the Pi Meson," Phys. Rev. 5, 1178-1184 (1958).

[41] S. Choi, V. Estenne, G. Bardin, N. De Botton, G. Fournier, P. A. M. Guichon, C. Marchand and J. Marroncle et al., "Axial and pseudoscalar nucleon form factors from low energy pion electroproduction," Phys. Rev. Lett. 71, 3927 (1993).

[42] A. Liesenfeld et al. [A1 Collaboration], "A measurement of the axial form factor of the nucleon by the $\mathrm{p}\left(\mathrm{e}, e^{\prime} \pi^{+}\right) \mathrm{n}$ reaction at $\mathrm{W}=1125 \mathrm{MeV}$," Phys. Lett. B 468, 20 (1999).

[43] M. N. Rosenbluth, "High Energy Elastic Scattering of Electrons on Protons," Phys. Rev. 79, 615619 (1950).

[44] A. Bodek, S. Avvakumov, R. Bradford and H. S. Budd, "Extraction of the axial nucleon form-factor from neutrino experiments on deuterium," J. Phys. Conf. Ser. 110, 082004 (2008) [arXiv:0709.3538 [hep-ex]].

[45] V. Lyubushkin, et al. "A study of quasi-elastic muon neutrino and antineutrino scattering in the NOMAD experiment." Eur. Phys. J. C63:355-381, (2009).

[46] A.A. Aguilar-Arevalo et al., "Measurement of Muon Neutrino Quasi-Elastic Scattering on Carbon," arXiv:0706.0926 [hep-ex], Phys. Rev. Lett. 100, 032301 (2008).

[47] A. A. Aguilar-Arevalo et al. [MiniBooNE Collaboration], "First Measurement of the Muon Neutrino Charged Current Quasielastic Double Differential Cross Section," Phys. Rev. D 81, 092005 (2010) [arXiv:1002.2680 [hep-ex]]. 
[48] O. Benhar, N. Farina, H. Nakamura, M. Sakuda, and R. Seki, "Electron- and neutrino-nucleus scattering in the impulse approximation regime," Phys. Rev. D 72, 053005 (2005).

[49] J. T. Sobczyk, "Multinucleon ejection model for Meson Exchange Current neutrino interactions," Phys. Rev. C 86, 015504 (2012) [arXiv:1201.3673 [hep-ph]].

[50] R. A. Smith, E. J. Moniz, "Neutrino Reactions On Nuclear Targets," Nucl. Phys. B43, 605 (1972).

[51] A. Bodek and J. L. Ritchie, "Fermi-motion effects in deep-inelastic lepton scattering from nuclear targets," Phys. Rev. D 23:5 1071-1091, (1981).

[52] A. M. Ankowski and J. T. Sobczyk, "Construction of spectral functions for medium-mass nuclei," Phys. Rev. C 77, 044311 (2008) [arXiv:0711.2031 [nuclth]].

[53] A. Bodek, H. S. Budd and M. E. Christy, "Neutrino Quasielastic Scattering on Nuclear Targets: Parametrizing Transverse Enhancement (Meson Exchange Currents)," Eur. Phys. J. C 71, 1726 (2011) [arXiv:1106.0340 [hep-ph]].

[54] J. Amaro, M. Barbaro, J. Caballero, T. Donnelly, and J. Udias, 2011b, Phys. Rev. D 84, 033004.

[55] M. Barbaro, et al., 2011, arXiv:1110.4739.

[56] C. Giusti, and A. Meucci, 2011, arXiv:1110.4005.

[57] M. Martini, M. Ericson, and G. Chanfray, 2011, Phys. Rev. C 84, 055502.

[58] J. Nieves, I. Simo, and M. Vacas, 2012, Phys. Lett. B 707, 72.

[59] M. Martini, M. Ericson, and G. Chanfray, 2011, Phys. Rev. C 84, 055502.

[60] J. T. Sobczyk, arXiv:1109.1081 (2012).

[61] C.D. Curtis, G.M. Lee, C.W. Owen, C.W. Schmidt and W.M. Smart, Linac Hbeam operation and uses at Fermilab, IEEE Trans. Nucl. Sci. 26 (1979) 3760.

[62] Stephen Holmes et al. "Overview of the Tevatron collider complex: goals, operations and performance," 2011 JINST 6 T08001.

[63] C.W. Schmidt, The Fermilab $400 \mathrm{MeV}$ Linac upgrade, in Proceedings of 1993 Particle Accelerator Conf., Washington U.S.A. (1993), pg. 1655.

[64] Detailed description of the Booster Beam in "Rookie Books," http://wwwbdnew.fnal.gov/operations/rookie_books/Booster_V4.1.pdf. 
[65] Detailed description of the Main Injector in "Rookie Books," http://wwwbdnew.fnal.gov/operations/rookie_books/Main_Injector_v1.1.pdf.

[66] NuMI Technical Design Handbook, Chapter 2 Overview of the NuMI Beamline, http://www-numi.fnal.gov/numwork/tdh/TDH_V2_2_Overview.pdf.

[67] A.G. Abramov et al. "Beam optics and target conceptual designs for the NuMI project," Nuclear Instruments and Methods in Physics Research A 485 (2002) 209227

[68] R. Zwaska et al. "Beam-based alignment of the NuMI target station components at FNAL," Nuclear Instruments and Methods in Physics Research A 568 (2006) 548560 .

[69] Z. Pavlovic, "Observation of Disappearance of Muon Neutrinos in the NuMI Beam," FERMILAB-THESIS-2008-59.

[70] S. E. Kopp, "The NuMI neutrino beam at Fermilab," arXiv:0508001.

[71] Detailed description of the NuMI Beam in "Rookie Books," wwwbdnew.fnal.gov/operations/rookie_books/NuMI_v1.pdf

[72] James Hylen, personal communication.

[73] S. Kopp et al. "Secondary beam monitors for the NuMI facility at FNAL," Nuclear Instruments and Methods in Physics Research A 568 (2006) 503519.

[74] "Extruded Plastic Scintillator for MINERvA," A. Pla-Dalmau, A. Bross, V. Rykalin and B. Wood, 2005 IEEE NSS Conference Record, FERMILAB-CONF05-506.

[75] A. Pla-Dalmau et al., Nuclear Instruments and Methods in Physics Research A 466 (2001) 482491.

[76] Kuraray Co. Ltd., "Scitntillation Materials" catalogue.

[77] http://sales.hamamatsu.com/en/products/electron-tubedivision/detectors/photomultiplier-tubes/part-r7600-00-m64.php, (2011).

[78] G. Perdue, et al., "The MINER $\nu$ A Data Acquisition System and Infrastructure," submitted to Nuclear Instruments and Methods in Physics Research A (2012).

[79] B. Baldin, MINERvA Chain Readout Controller, MINERvA Doc-DB 1516, 2007. 
[80] B. Baldin, VME Data Acquisition Modules for MINERvA Experiment, FERMILAB-TM-2458-PPD, 2010.

[81] B. Baldin, MINERvA CROC Interface Module, MINERvA Doc-DB 1238, 2009.

[82] The CAEN v2718 VME Crate Controller ¡http://www.caen.it/nuclear/product.php?mod=V2718¿, 2008.

[83] T. Fitzpatrick, C. Rotolo, MINOS Master Clock System Preliminary Design Specification, NUMI-NOTR-ELEC-827, Fermilab, 2000.

[84] Windows Vista. http://windows.microsoft.com/en-US/windowsvista/products/home; 2010.

[85] Fermi Linux. http://fermilinux.fnal.gov/; 2011.

[86] T. Cundiff, et al., "The MINOS near detector front end electronics," IEEE Trans. Nucl. Sci. 53, 1347-1355 (2006).

[87] D.G. Michael et al., "The magnetized steel and scintillator calorimeters of the MINOS experiment," Fermilab-Pub-08-126, Nucl.Instrum.Meth.A596:190$228(2008)$.

[88] C. Anderson et al., "The ArgoNeuT Detector in the NuMI Low-Energy beam line at Fermilab," http://arxiv.org/abs/1205.6747 (2012).

[89] C.Andreopoulos, A.Bell, D.Bhattacharya, F.Cavanna, J.Dobson, S.Dytman, H.Gallagher, R.Hatcher, P.Kehayias, A.Meregaglia, D.Naples, G.Pearce, A.Rubbia, M.Whalley, T.Yang, "The GENIE Neutrino Monte Carlo Generator", Nucl.Instrum.Meth.A614:87-104,2010.

[90] J. Allison et al., IEEE Transactions on Nuclear Science 53 No. 1 (2006) 270-278.

[91] A. M. McGowan, "Observation of deficit in NuMI neutrino-induced rock and non-fiducial muons in MINOS Far Detector and measurement of neutrino oscillation parameters," FERMILAB-THESIS-2007-31.

[92] C. Alt, B. Baatar, D. Barna, G. Barr, J. Bartke, L. Betev, H. Biakowska, C. Blume, B. Boimska and J. Bracinik, et al. "Inclusive production of charged pions in $\mathrm{p}+\mathrm{C}$ collisions at $158 \mathrm{GeV} / \mathrm{c}$ beam momentum," NA Collaboration. Eur. Phys. J. C 49, 897917 (2007).

[93] D.S. Barton et al. "Experimental study of the A dependence of inclusive hadron fragmentation," Phys. Rew. D Vol27 N 11.

[94] ROOT Collaboration, http://root.cern.ch. 
[95] R. Bradford, A. Bodek, H. Budd and J. Arrington, "A new parameterization of the nucleon elastic form factors," Nucl. Phys. Proc. Suppl. 159, 127 (2006) [arXiv:hepex/0602017].

[96] D. Rein and L. M. Sehgal, "Neutrino Excitation Of Baryon Resonances And Single Pion Production," Annals Phys. 133, 79 (1981).

[97] A. Bodek, I. Park and U. -k. Yang, "Improved low Q**2 model for neutrino and electron nucleon cross sections in few GeV region," Nucl. Phys. Proc. Suppl. 139, 113 (2005).

[98] R.E. Hendrick, L. Li, "Gauge-model constraints of recent elastic and deepinelastic neutral-current data," Phys. Rev. D19, 779, (1979).

[99] J.B. Birks, Proc. Phys. Soc. A64: 874 (1951).

[100] Autodesk (2011). AutoCAD [Computer program]. Available at: http://usa.autodesk.com/autocad/ (Accessed 5 July 2011).

[101] "Geometry Description Markup Language for Physics Simulation and Analysis Applications," R. Chytracek, J. McCormick, W. Pokorski, G. Santin, IEEE Trans. Nucl. Sci., Vol. 53, Issue: 5, Part 2, 2892-2896

[102] J. W. J. Williams. "Algorithm 232 - Heapsort," Communications of the ACM 7(6): 347348, (1964).

[103] S. Carlsson, "Average-case results on heapsort," BIT 27(1): 2-17, (1987).

[104] N. Tagg, et al., "Arachne - A web-based event viewer for MINER $\nu$ A," Nucl.Inst.Meth. 676 (2012) 44-49.

[105] R. Fruhwirth, "Application of Kalman Filtering to Track and Vertex Fitting," Nucl.Instrum.Meth. A262 444-450, (1987).

[106] W. D. Hulsbergen, "Decay Chain Fitting with a Kalman Filter," Nucl.Instrum.Meth. A552 566-575 (2005).

[107] R. J. Barlow and C. Beeston, Comput. Phys. Commun. 77, 219 (1993).

[108] M. Lefebvre, R.K. Keeler, R. Sobie, J. White, "Propagation of Errors for Matrix Inversion," Nucl.Instrum.Meth. A451 (2000) 520-528.

[109] J. Bell, C. T. Coffin, R. N. Diamond, H. T. French, W. C. Louis, B. P. Roe, R. T. Ross and A. A. Seidl et al., "A Study Of The Reaction Neutrino P $\rightarrow$ Mu- Delta++ At High-energies And Comparisons With Theory," Phys. Rev. Lett. 41, 1012 (1978). 
[110] Hugh Gallagher, personal communication.

[111] Zanelli et al., Phys rev C23, 1915 (1981).

[112] Schimmerling et al., Phys Rev C7, 248 (1973).

[113] Voss et al., Proc. Royal Soc. London A236, 52 (1956).

[114] Ibaraki et al., Jour. Nucl. Sci. Tech. Supp. 2, 405 (2002).

[115] Abfalterer et al., Phys Rev C63, 044608 (2001).

[116] Dietrich et al., Jour. Nucl. Sci. Tech. Supp. 2, 269 (2002).

[117] Menet et al., Phys Rev C4, 1114 (1971).

[118] McGill et al., Phys Rev C19, 2237 (1974).

[119] Renberg et al., Nucl Phys A183, 81 (1972).

[120] Dicello et al., Phys Rev 157, 1001 (1967).

[121] MacGregor et al., Phys Rev 111, 1155 (1958).

[122] Bauhof et al., At. Data and Nucl. Data Tab. 35, 429 (1990).

[123] Ashery et al., Phys Rev C23, 2173 (1981).

[124] Wilkin et al., Nucl Phys B62, 61 (1971).

[125] Clough et al., Nucl Phys B76, 15 (1974).

[126] Alderdyce et al., Nucl Phys A209, 1, (1973).

[127] Gelderloos et al., Phys Rev C62, 024612 (2000).

[128] William H. Press, Saul A. Teukolsky, William T. Vetterling, and Brian P. Flannery, "Numerical Recipes 3rd Edition: The Art of Scientific Computing," Cambridge University Press, (2007).

[129] T.Golan, C. Juszczak, J. Sobczyk, "Final State Interactions Effects in Neutrino-Nucleus Interactions," arXiv:1202.4197.

[130] http://www2.dupont.com/Tedlar_PVF_Film/en_US/index.html.

[131] http://www.eljentechnology.com/index.php/joomla-overview/paints/86-ej510 . 
[132] http://www.momentive.com/Products/TechnicalDataSheet.aspx?id=3994.

[133] N. Tagg et al., Nuclear Instruments and Methods in Physics Research A 539 (2005) 668678.

[134] http://www.tek.com/datasheet/data-pattern-generator.

[135] J. Estrada, C. Garcia, B. Hoeneisen, and P. Rubinov, "MCM II and the Trip chip“, FERMILAB-TM-2226; (2004).

[136] http://msdn.microsoft.com/en-us/vstudio/hh388573.aspx.

[137] http://windows.microsoft.com/en-US/windows/products/windows-xp.

[138] http://motion.kodak.com/motion/Products/Lab_And_Post_Production/Kod ak_filters/wrattten2.htm. 


\section{Appendix A}

\section{Reconstruction and Resolution Plots for the CCQE Analysis}

This appendix contains data/simulation overlays, data/simulation ratio plots, and resolution plots for the CCQE analysis presented in Chapter 5. All plots have all CCQE selection cuts applied. For the resolution plots, in addition to having CCQE selection cuts applied, we also require that events be true CCQE events. If a particular plot is shown in Chapter 5 , it is not shown again in this appendix. For most quantities, we also show absolutely normalized data and simulation overlays with systematic errors applied. These plots demonstrate that we see good agreement between data and the simulation and that we find no major issues in the simulation that are not already addressed by our assessment of systematic errors given in Section 5.3. For all ratio plots shown in this appendix, the $\chi^{2}$ value is for the agreement of the ratio with a flat line.

We show resolution plots for vertex, angle, muon energy, and $\mathrm{Q}_{\mathrm{QE}}^{2}$. For angle resolution plots, we find resolutions for the angle of the muon with respect to the 
incoming neutrino as well as the $\mathrm{X}$ and $\mathrm{Y}$ components of that angle. This is to ensure that no major reconstruction issues are present in one particular direction. Resolution plots are shown in Figures A.11, A.12, A.13, A.14, A.18, A.19, A.20, A.21, A.22, A.23, and A.24. We list the resolutions for these quantities in Tables A.1, A.2, A.3, A.4, A.5, and A.6.
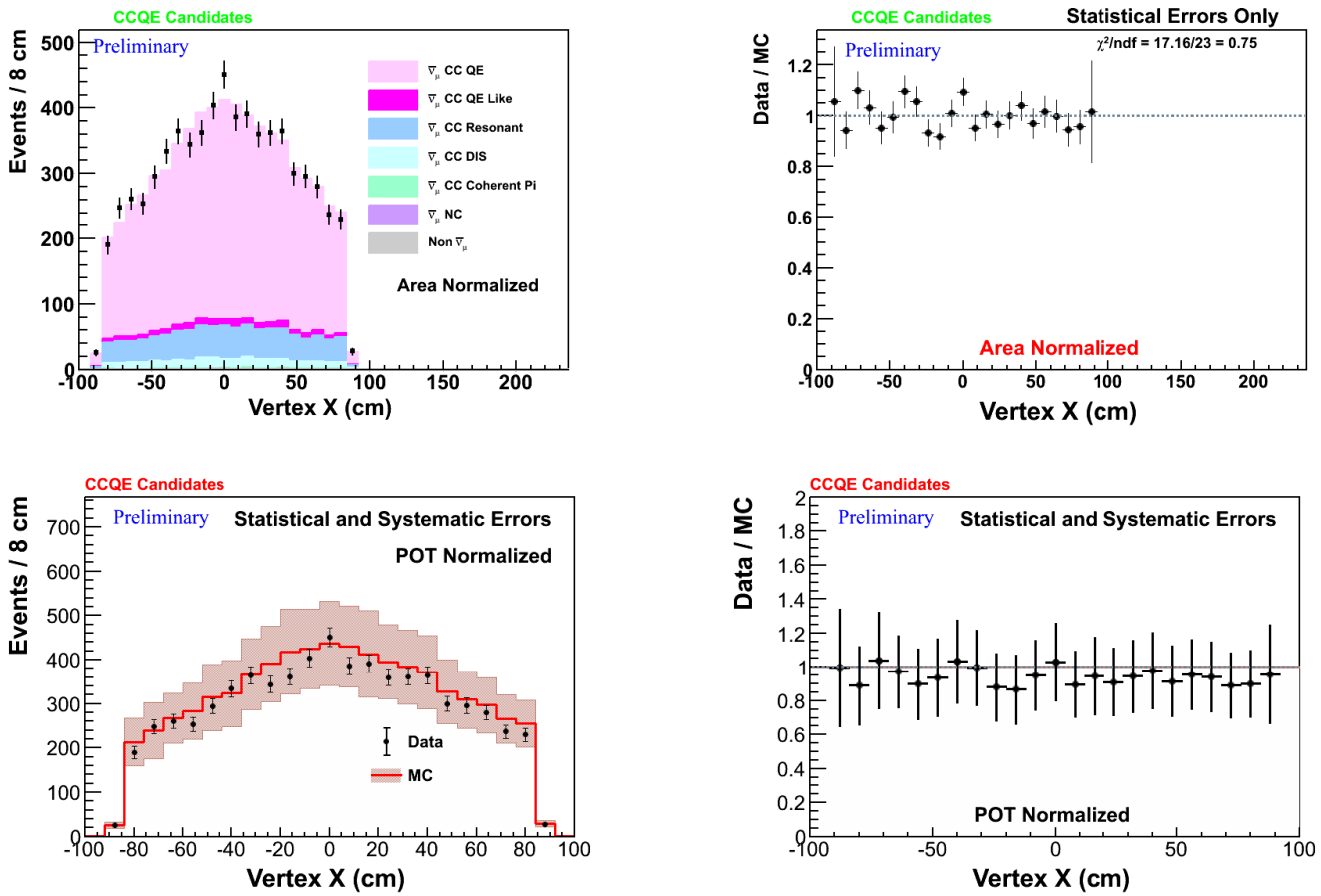

Figure A.1: Distributions of the $\mathrm{X}$ vertex for the CCQE sample. Above is the area normalized data/MC overlay and ratio. The overlay shows the composition of signal and background according to the simulation. Below is an absolutely normalized distribution with all relevant systematic errors applied. 

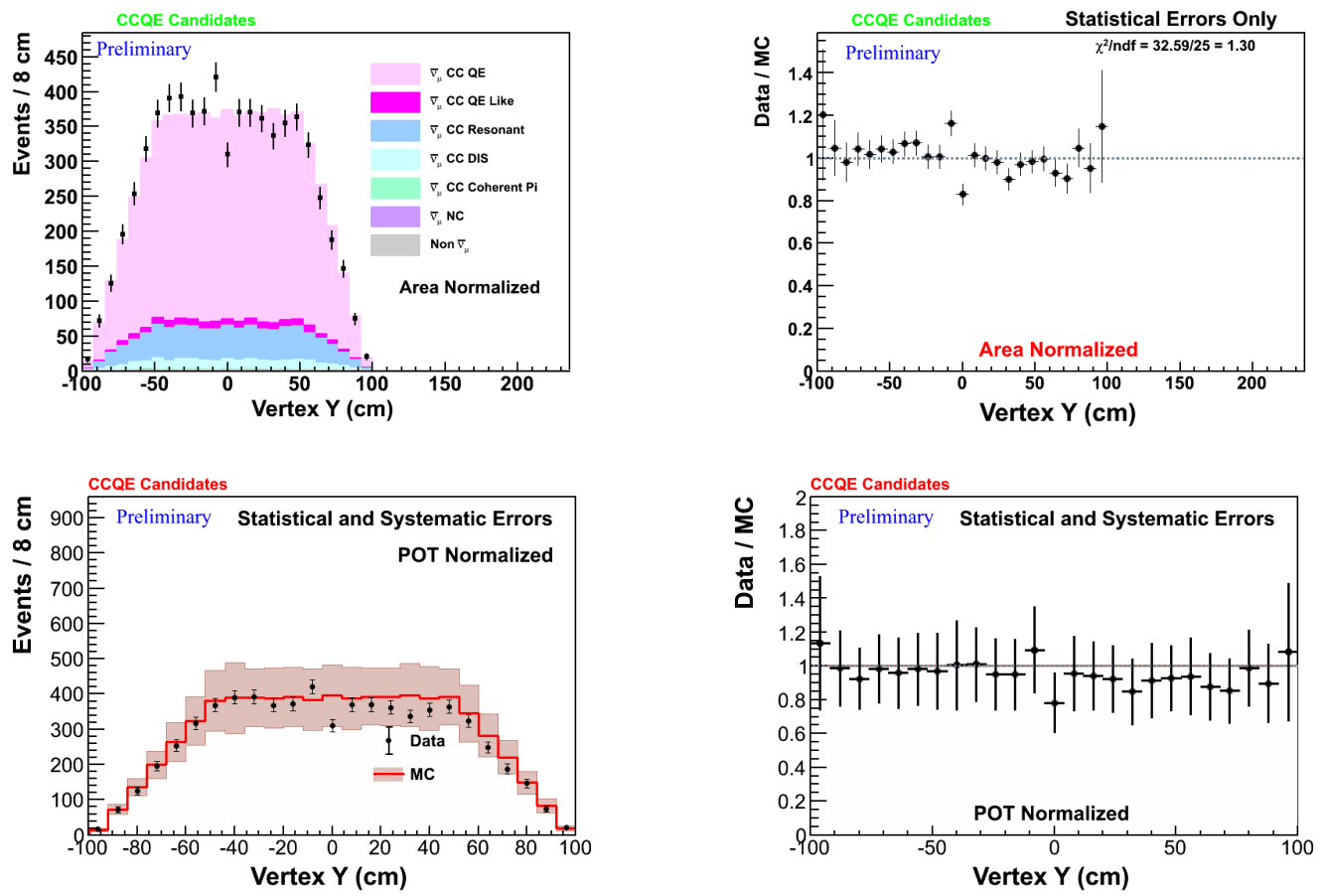

Figure A.2: Distributions of the $\mathrm{Y}$ vertex for the CCQE sample. Above is the area normalized data/MC overlay and ratio. The overlay shows the composition of signal and background according to the simulation. Below is an absolutely normalized distribution with all relevant systematic errors applied. 

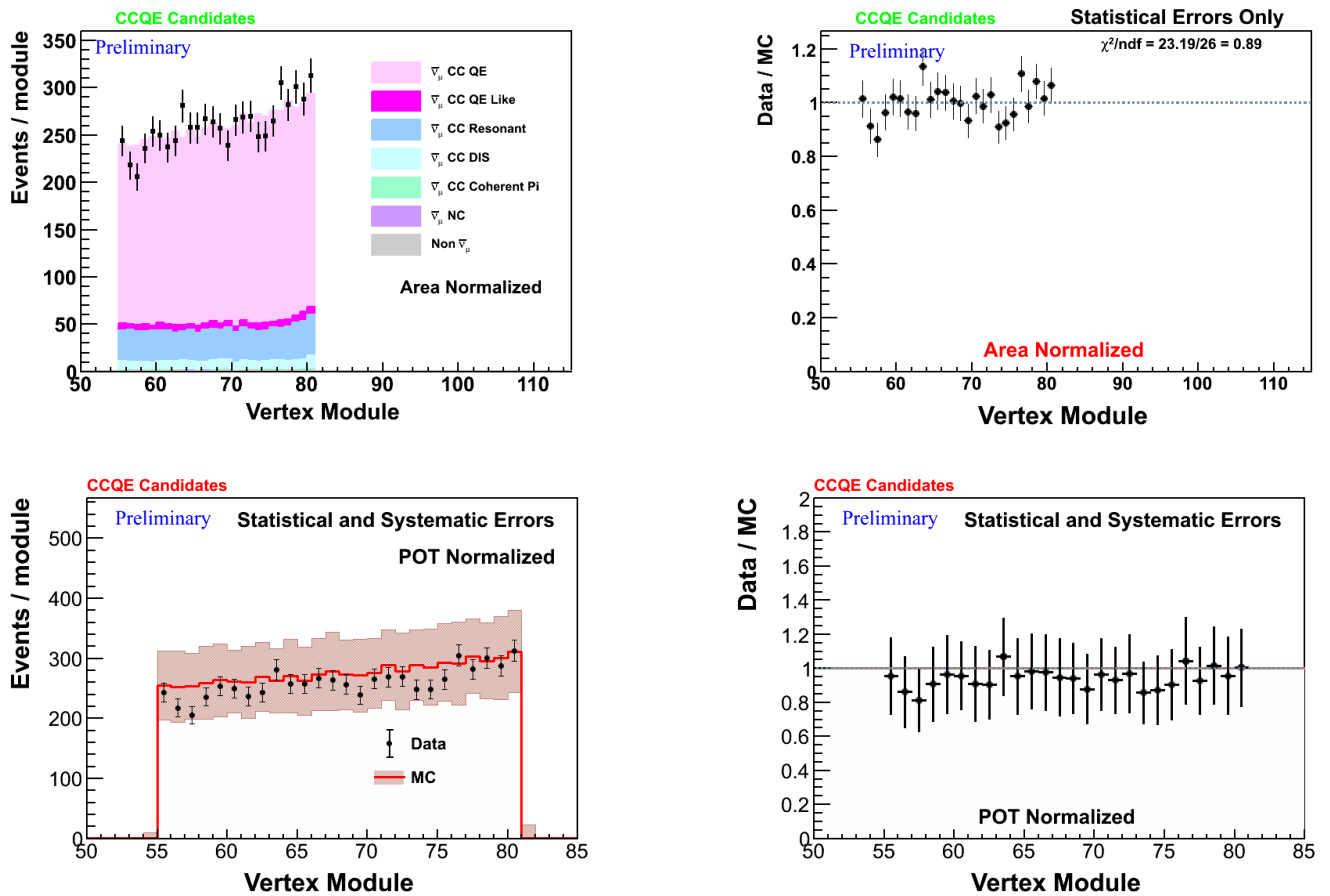

Figure A.3: Distributions of the vertex as function of module for the CCQE sample. Above is the area normalized data/MC overlay and ratio. The overlay shows the composition of signal and background according to the simulation. Below is an absolutely normalized distribution with all relevant systematic errors applied. 

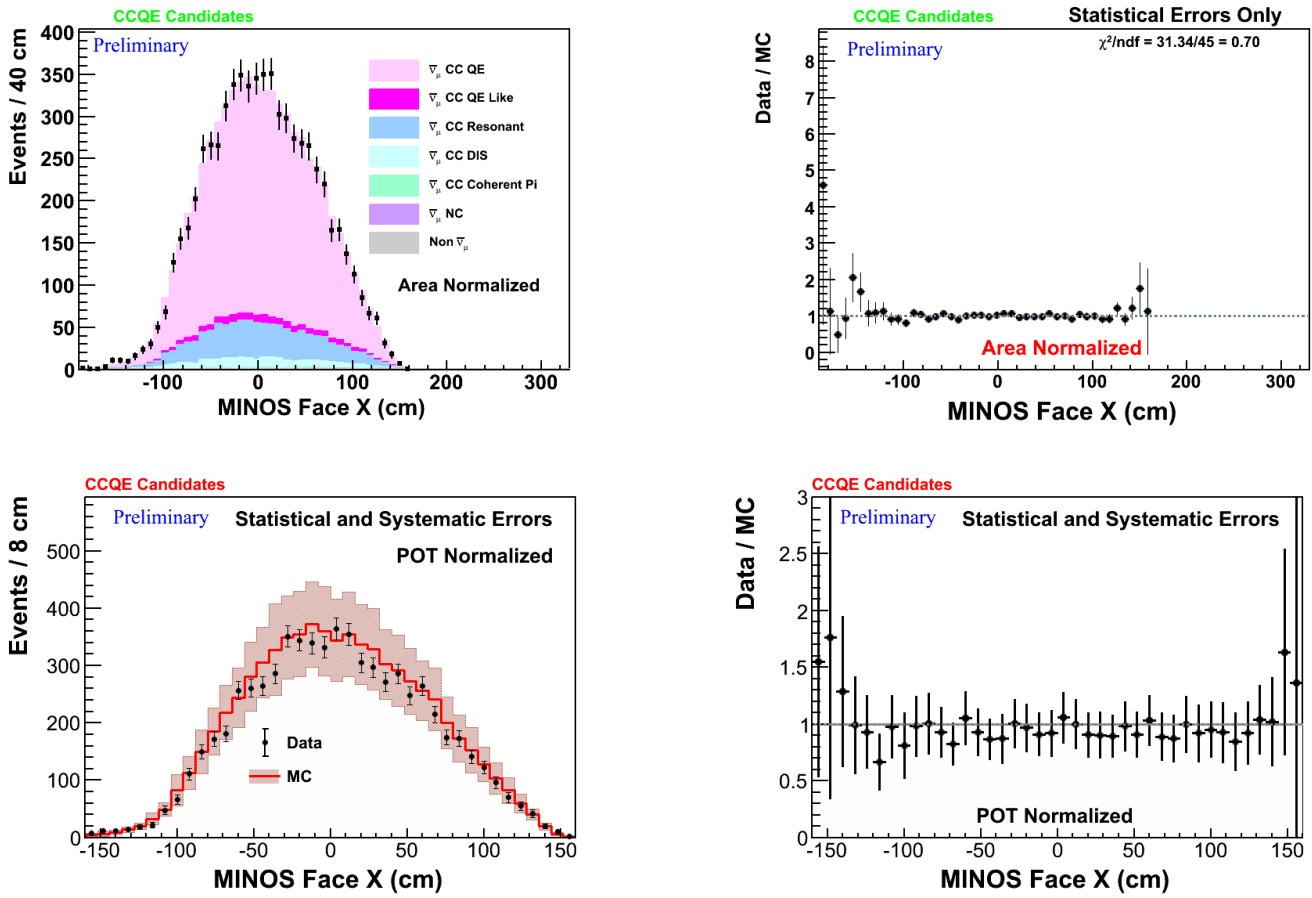

Figure A.4: Distributions of the $\mathrm{X}$ coordinate of the projection of the MINER $\nu \mathrm{A}$ track to the face of MINOS for the CCQE sample. Above is the area normalized data/MC overlay and ratio. The overlay shows the composition of signal and background according to the simulation. Below is an absolutely normalized distribution with all relevant systematic errors applied. 

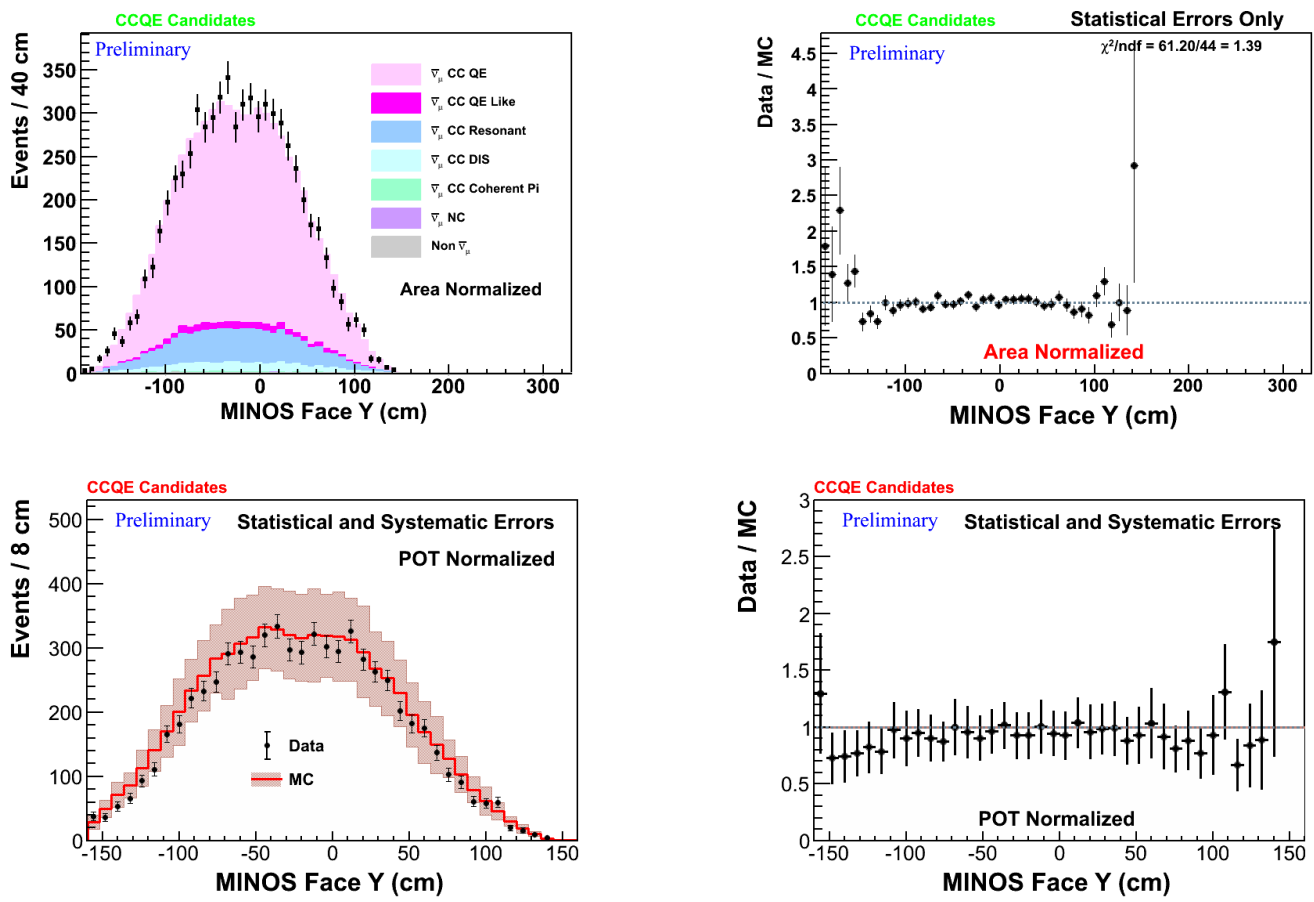

Figure A.5: Distributions of the Y coordinate of the projection of the MINER $\nu \mathrm{A}$ track to the face of MINOS for the CCQE sample. Above is the area normalized data/MC overlay and ratio. The overlay shows the composition of signal and background according to the simulation. Below is an absolutely normalized distribution with all relevant systematic errors applied.
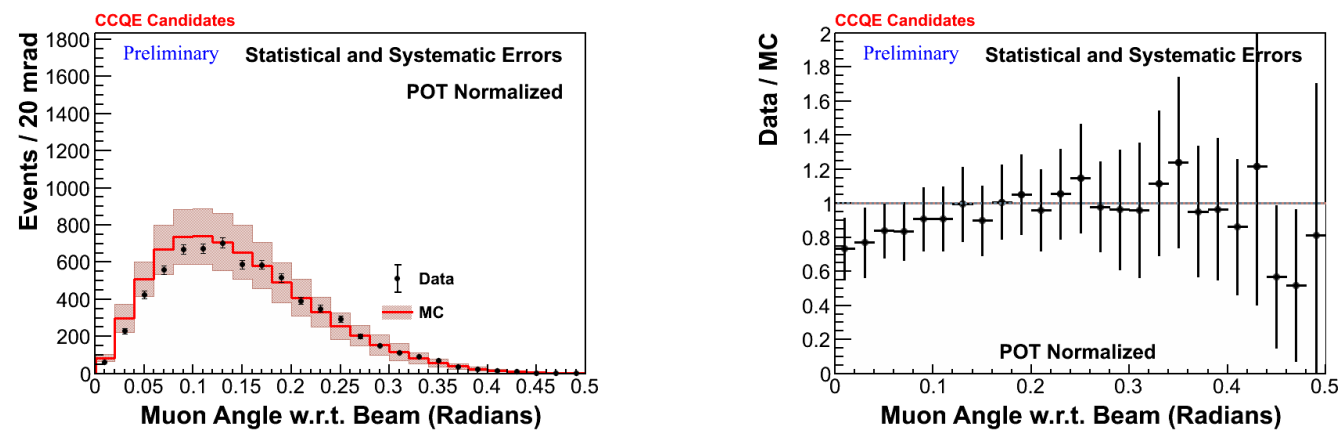

Figure A.6: Absolutely normalized muon angle distributions with statistical and systematic errors. 

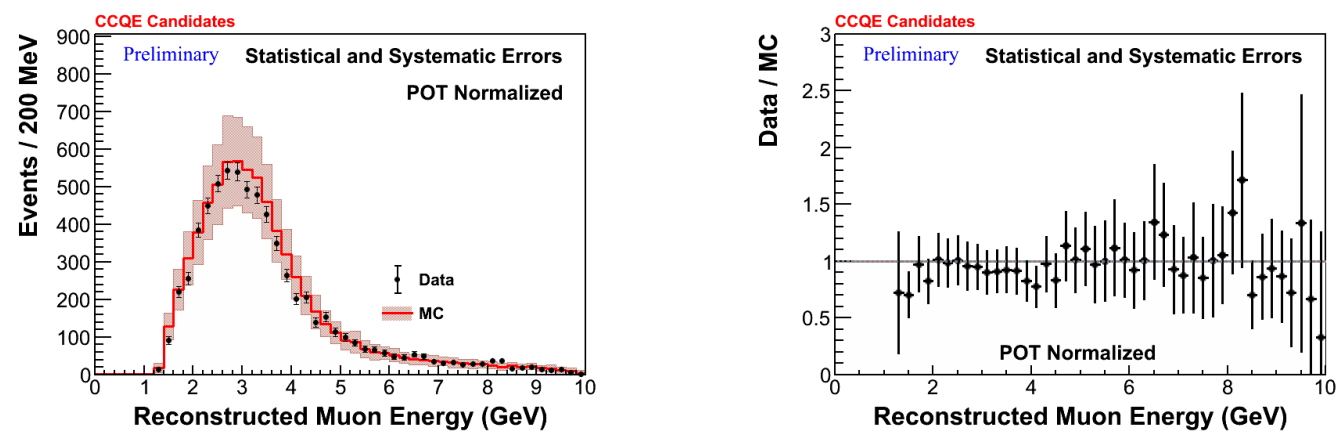

Figure A.7: Absolutely normalized muon energy overlay and ratio plots with statistical and systematic errors.
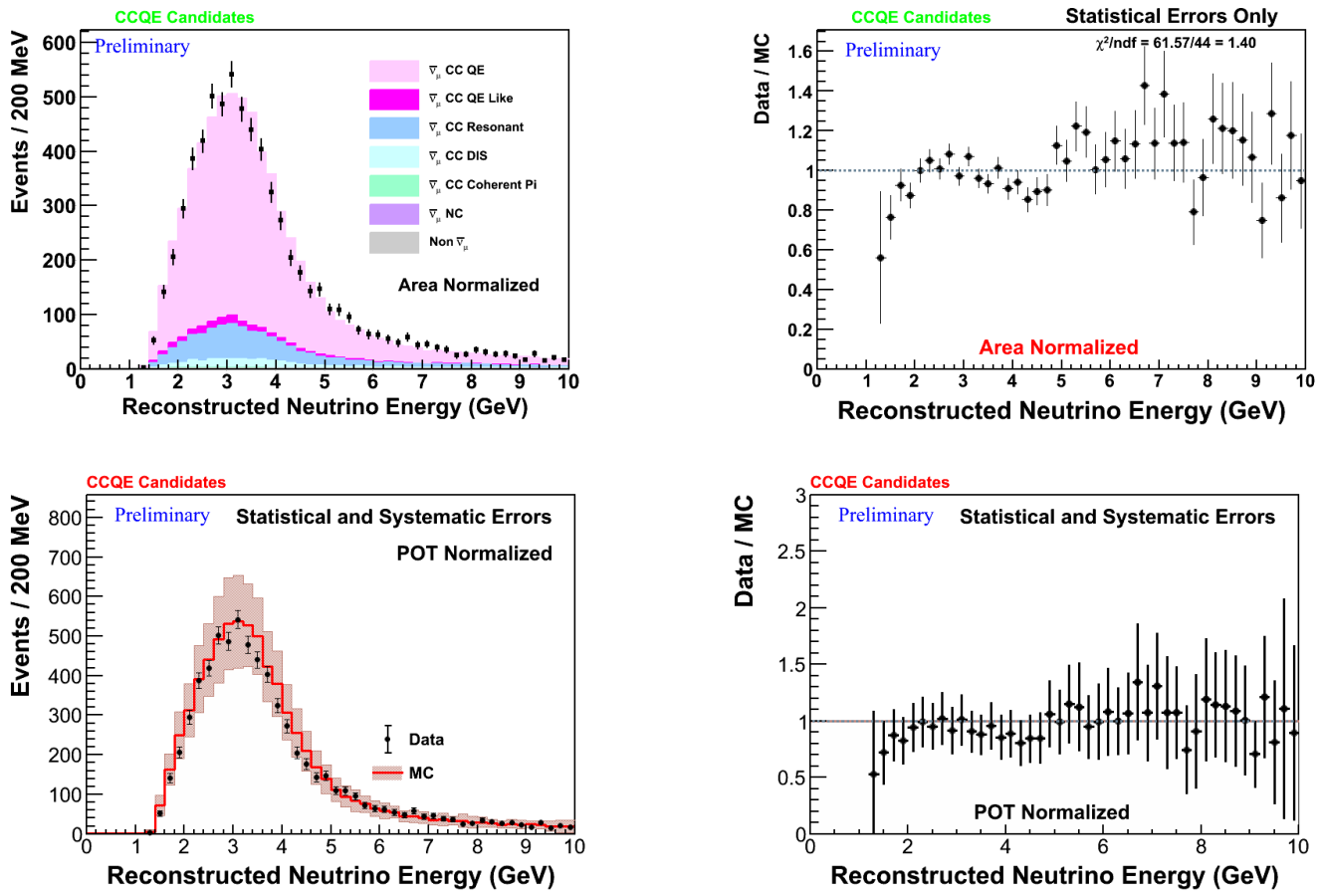

Figure A.8: Distributions of neutrino energy for the CCQE sample. Above is the area normalized data/MC overlay and ratio. The overlay shows the composition of signal and background according to the simulation. Below is an absolutely normalized distribution with all relevant systematic errors applied. 

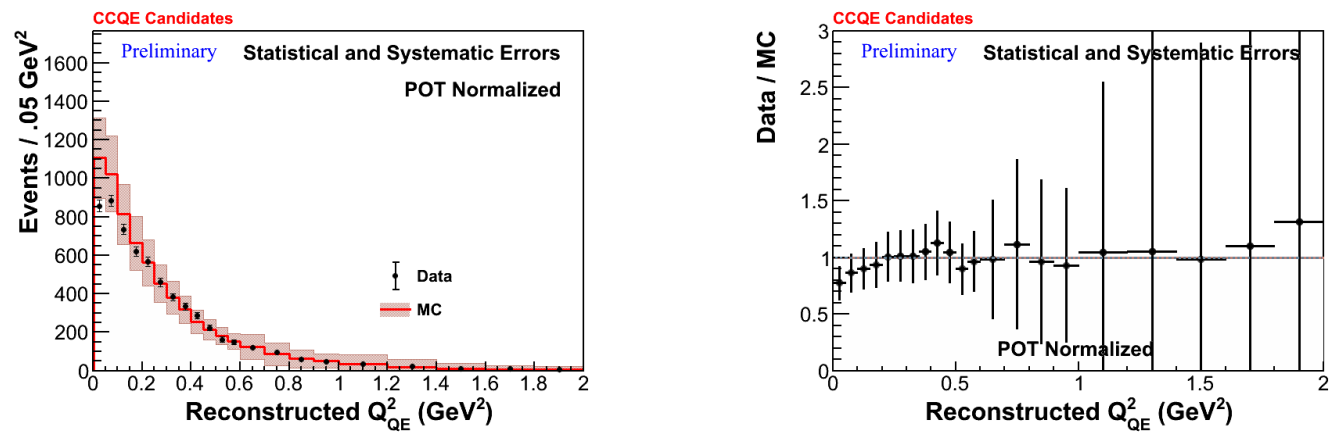

Figure A.9: Absolutely normalized $\mathrm{Q}_{\mathrm{QE}}^{2}$ overlay and ratio plots with statistical and systematic errors.
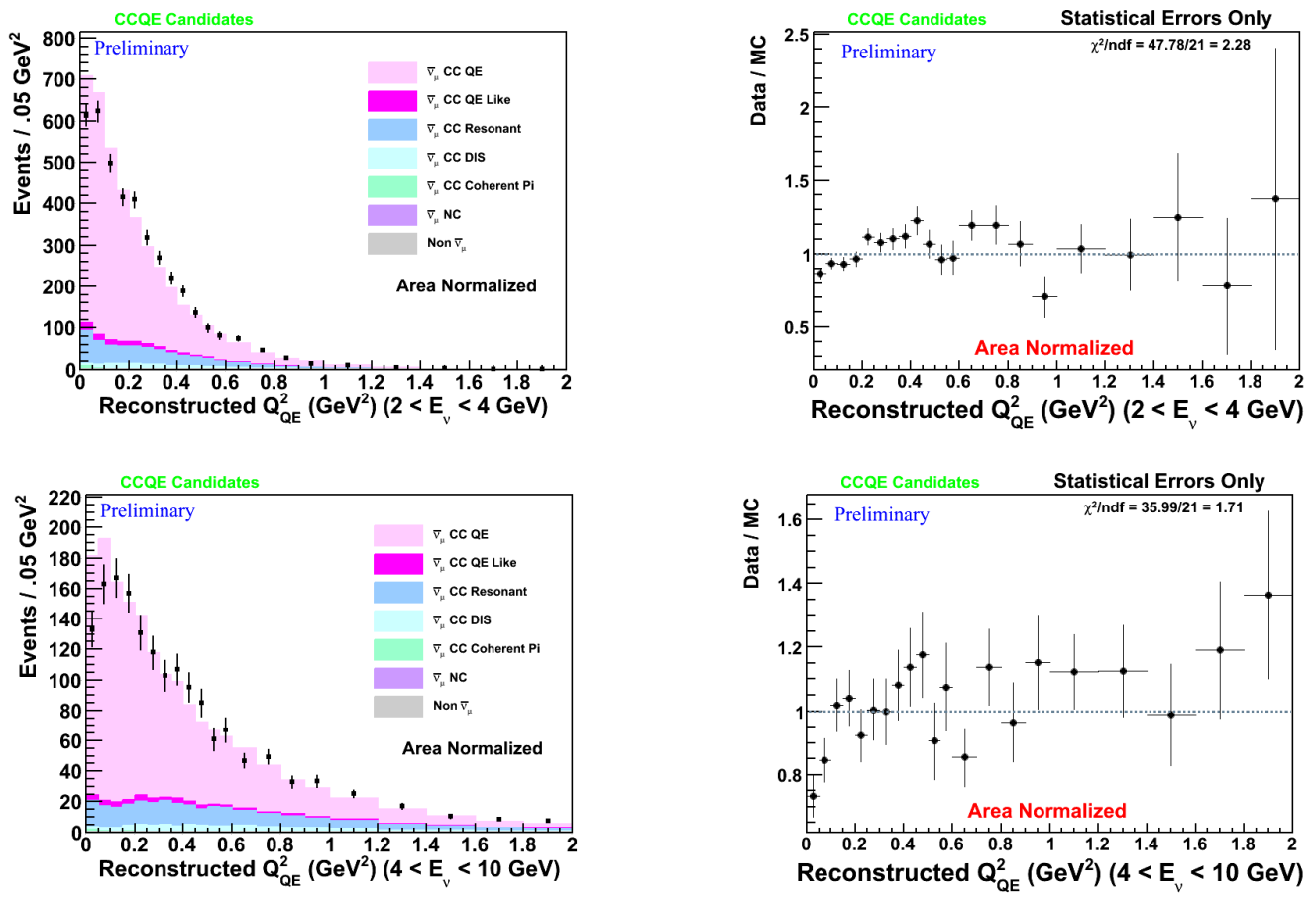

Figure A.10: $\mathrm{Q}_{\mathrm{QE}}^{2}$ overlays and ratios for two different ranges of neutrino energy. Events in both energy ranges have similar shape disagreement between data and simulation at low $\mathrm{Q}_{\mathrm{QE}}^{2}$. Note that the $\mathrm{Q}_{\mathrm{QE}}^{2}$ for neutrino energies between 2 and $4 \mathrm{GeV}$ is narrower due to the kinematic limit of $\mathrm{Q}_{\mathrm{QE}}^{2}$. 


\begin{tabular}{c|c}
\hline \hline Direction & Vertex Resolution $(\mathrm{mm})$ \\
\hline $\mathrm{X}$ & 2.87 \\
$\mathrm{Y}$ & 3.14 \\
\hline \hline
\end{tabular}

Table A.1: $\mathrm{X}$ and $\mathrm{Y}$ vertex resolution.
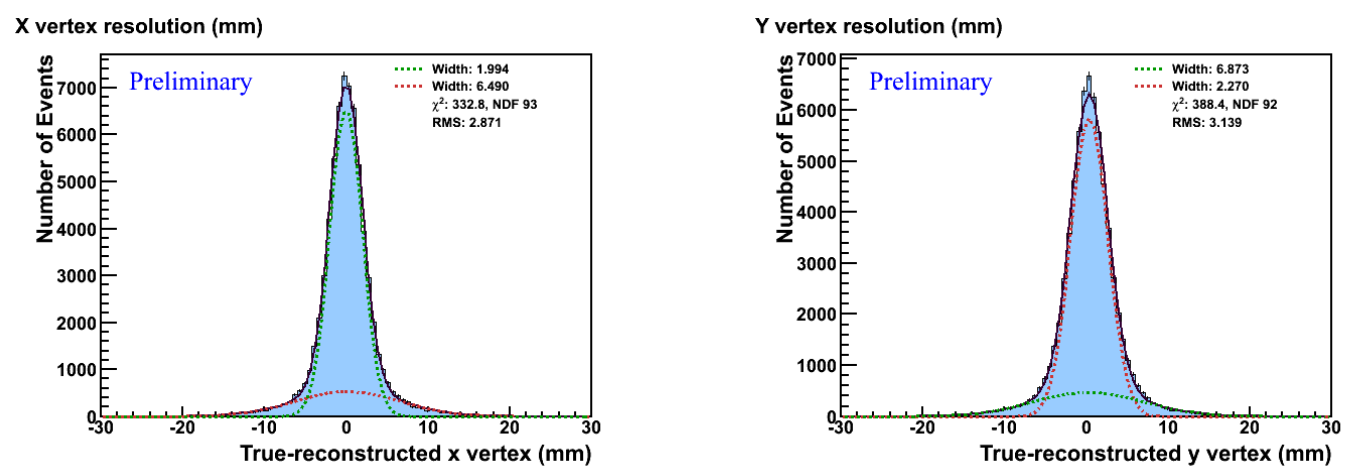

Figure A.11: X and Y vertex resolution plots for the CCQE sample with a double gaussian fit. The overall vertex resolution is roughly $3 \mathrm{~mm}$ for both the $\mathrm{X}$ and $\mathrm{Y}$ coordinate. Plots made by $\mathrm{C}$. Patrick of the MINER $\nu \mathrm{A}$ collaboration. 


\begin{tabular}{c|c|c}
\hline \hline$\theta_{\mu}($ degrees $)$ & $\mathrm{E}_{\mu}(\mathrm{GeV})$ & $\mathrm{E}_{\mu}$ Resolution $(\mathrm{GeV})$ \\
\hline$[0,5)$ & {$[0,3)$} & 0.227 \\
{$[5,10)$} & {$[0,3)$} & 0.237 \\
{$[10, \infty)$} & {$[0,3)$} & 0.283 \\
{$[0,5)$} & {$[3,5)$} & 0.338 \\
{$[5,10)$} & {$[3,5)$} & 0.343 \\
{$[10, \infty)$} & {$[3,5)$} & 0.389 \\
{$[0,5)$} & {$[5, \infty)$} & 0.667 \\
{$[5,10)$} & {$[5, \infty)$} & 0.603 \\
{$[10, \infty)$} & {$[5, \infty)$} & 0.515 \\
\hline \hline
\end{tabular}

Table A.2: Muon energy resolution binned by muon energy and angle.
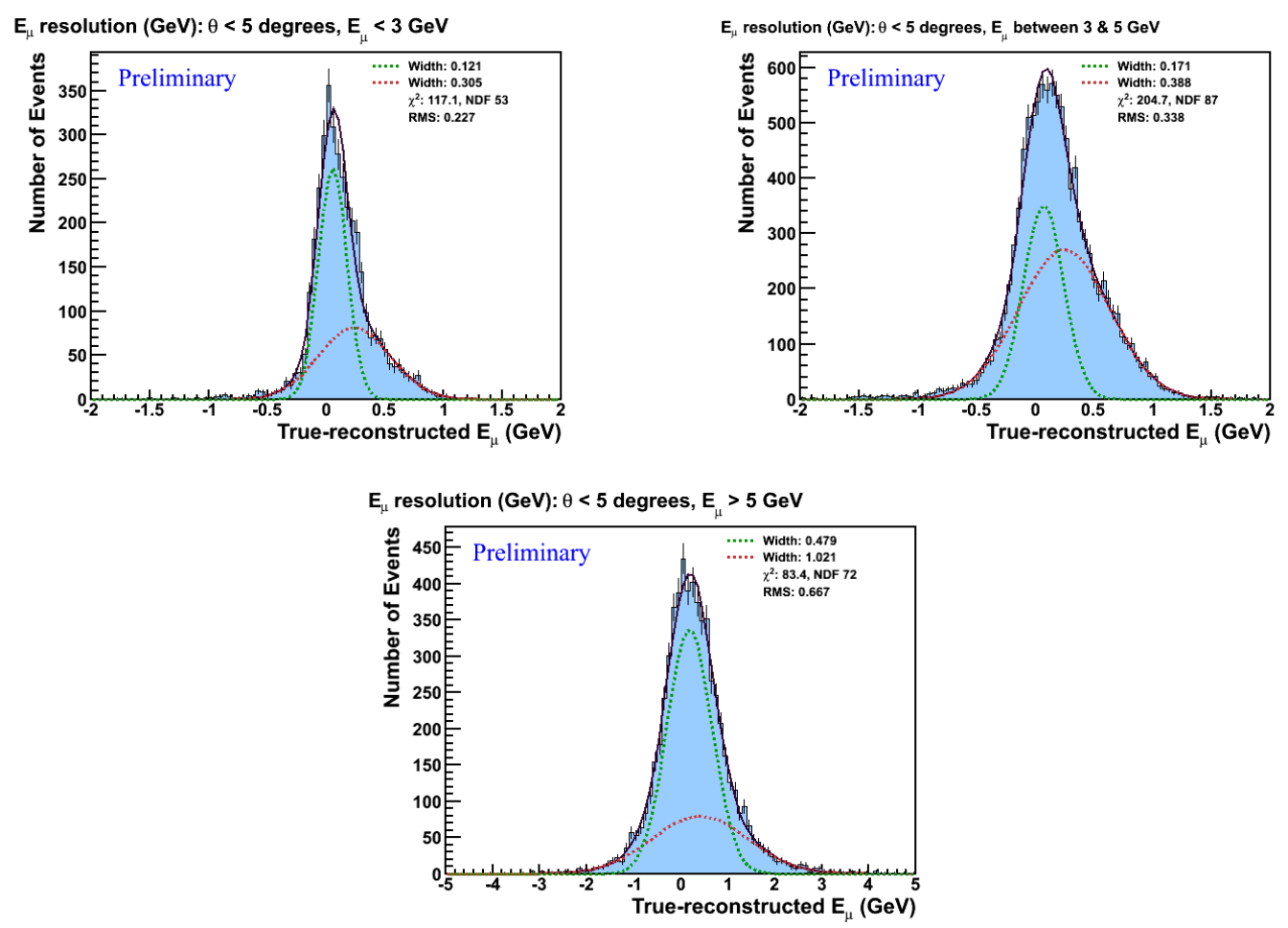

Figure A.12: Muon energy resolution plots for muons with angles from 0 to 5 degrees. We consider the resolution for muons with energies less than $3 \mathrm{GeV}$, between $3-5 \mathrm{GeV}$, and greater than $5 \mathrm{GeV}$. Plots made by $\mathrm{C}$. Patrick of the MINER $\nu \mathrm{A}$ collaboration. 

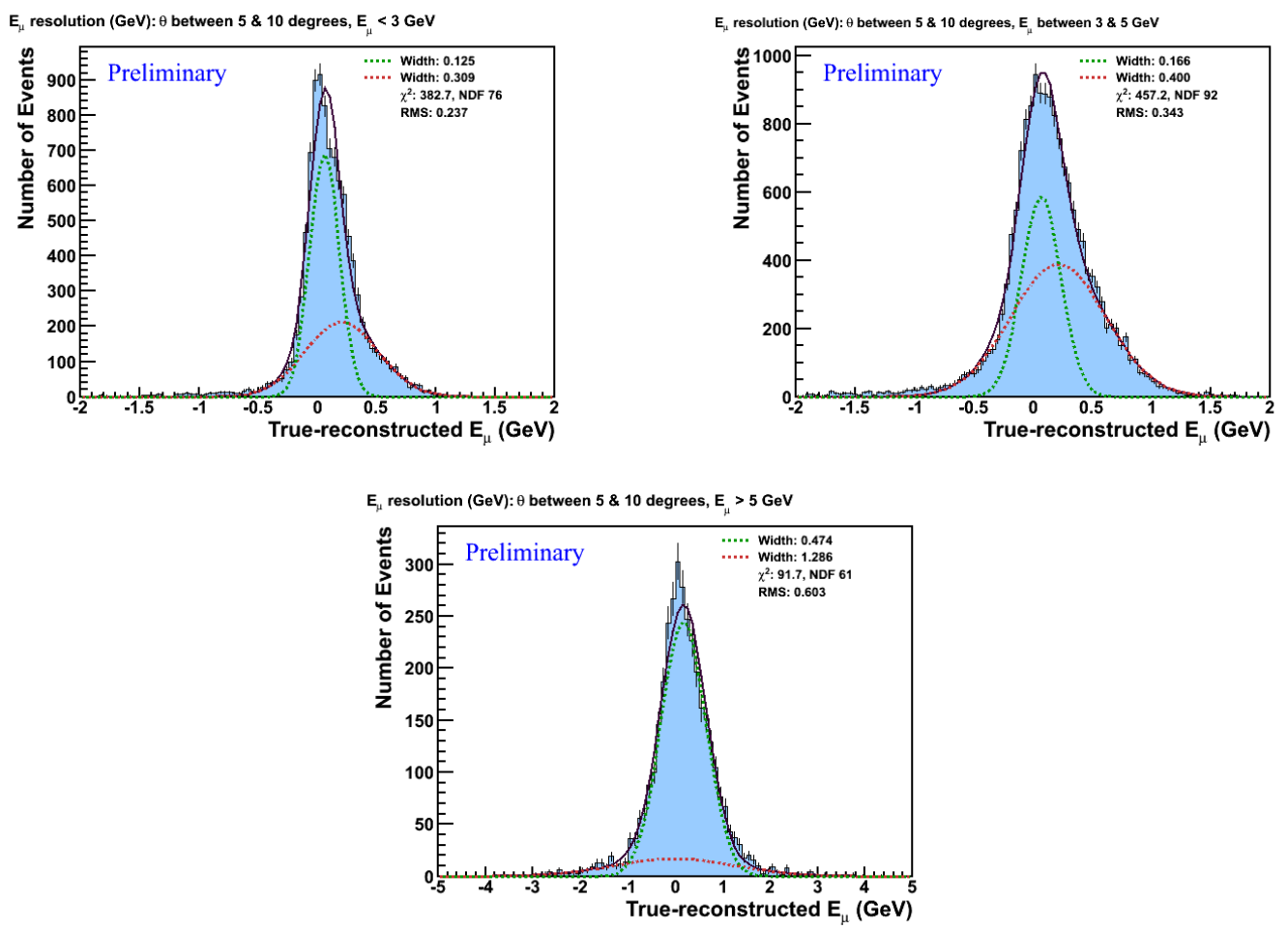

Figure A.13: Muon energy resolution plots for muons with angles from 5 to 10 degrees. We consider the resolution for muons with energies less than $3 \mathrm{GeV}$, between $3-5 \mathrm{GeV}$, and greater than $5 \mathrm{GeV}$. Plots made by C. Patrick of the MINER $\nu$ A collaboration. 

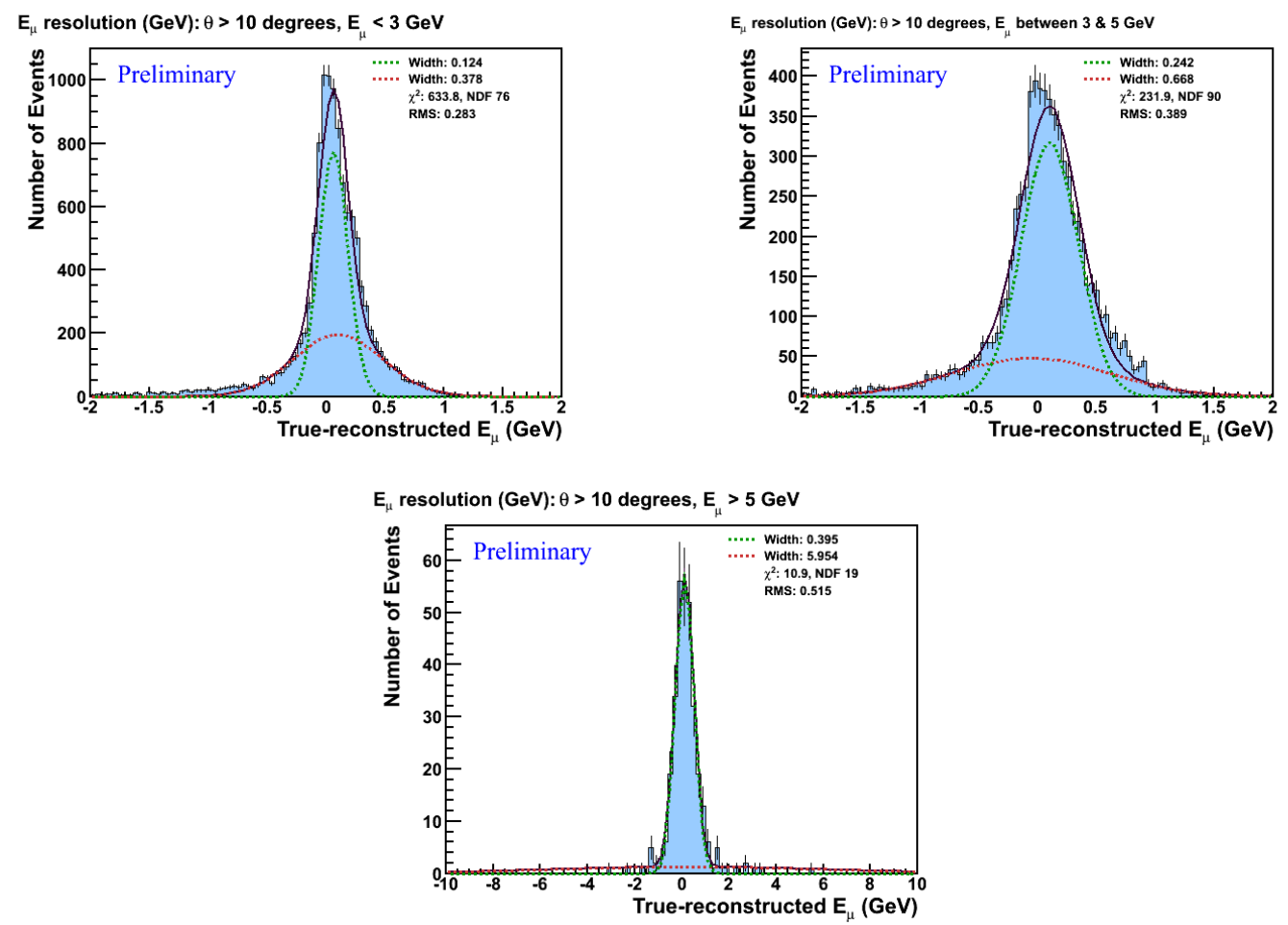

Figure A.14: Muon energy resolution plots for muons with angles from 0 to 5 degrees. We consider the resolution for muons with energies less than $3 \mathrm{GeV}$, between $3-5 \mathrm{GeV}$, and greater than $5 \mathrm{GeV}$. Plots made by $\mathrm{C}$. Patrick of the MINER $\nu \mathrm{A}$ collaboration. 


\begin{tabular}{c|c|c}
\hline \hline$\theta_{\mu}$ (degrees) & $\mathrm{E}_{\mu}(\mathrm{GeV})$ & $\theta_{\mu}$ Resolution (degrees) \\
\hline$[0,5)$ & {$[0,3)$} & 0.432 \\
{$[5,10)$} & {$[0,3)$} & 0.494 \\
{$[10, \infty)$} & {$[0,3)$} & 0.563 \\
{$[0,5)$} & {$[3,5)$} & 0.396 \\
{$[5,10)$} & {$[3,5)$} & 0.477 \\
{$[10, \infty)$} & {$[3,5)$} & 0.516 \\
{$[0,5)$} & {$[5, \infty)$} & 0.42 \\
{$[5,10)$} & {$[5, \infty)$} & 0.515 \\
{$[10, \infty)$} & {$[5, \infty)$} & 0.514 \\
\hline \hline
\end{tabular}

Table A.3: Muon angle resolution binned by muon energy and angle.
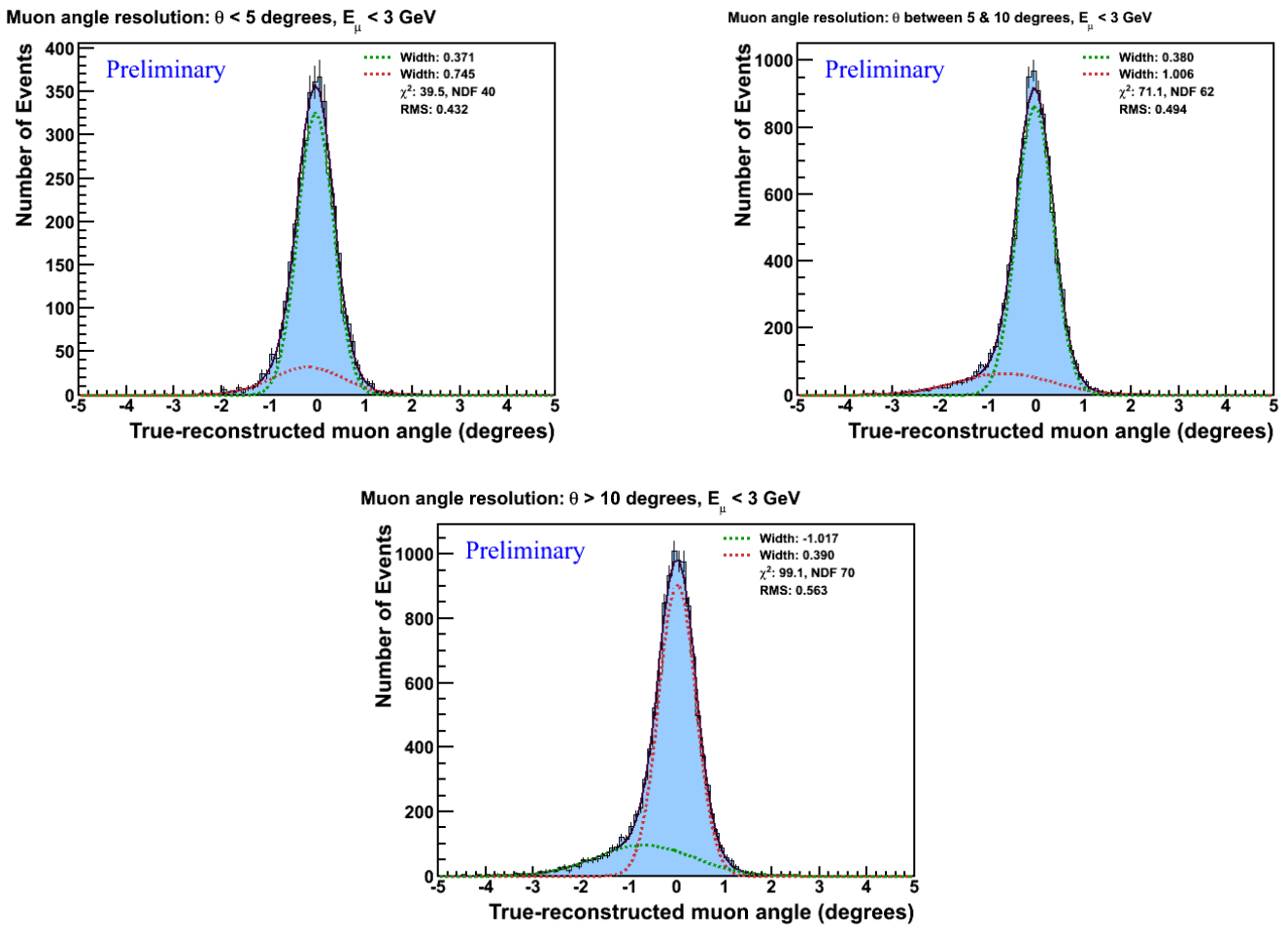

Figure A.15: Muon angle resolution for muons with an energy of 0 to $3 \mathrm{GeV}$. We consider the resolution for different ranges of angle. Plots made by C. Patrick of the MINER $\nu \mathrm{A}$ collaboration. 

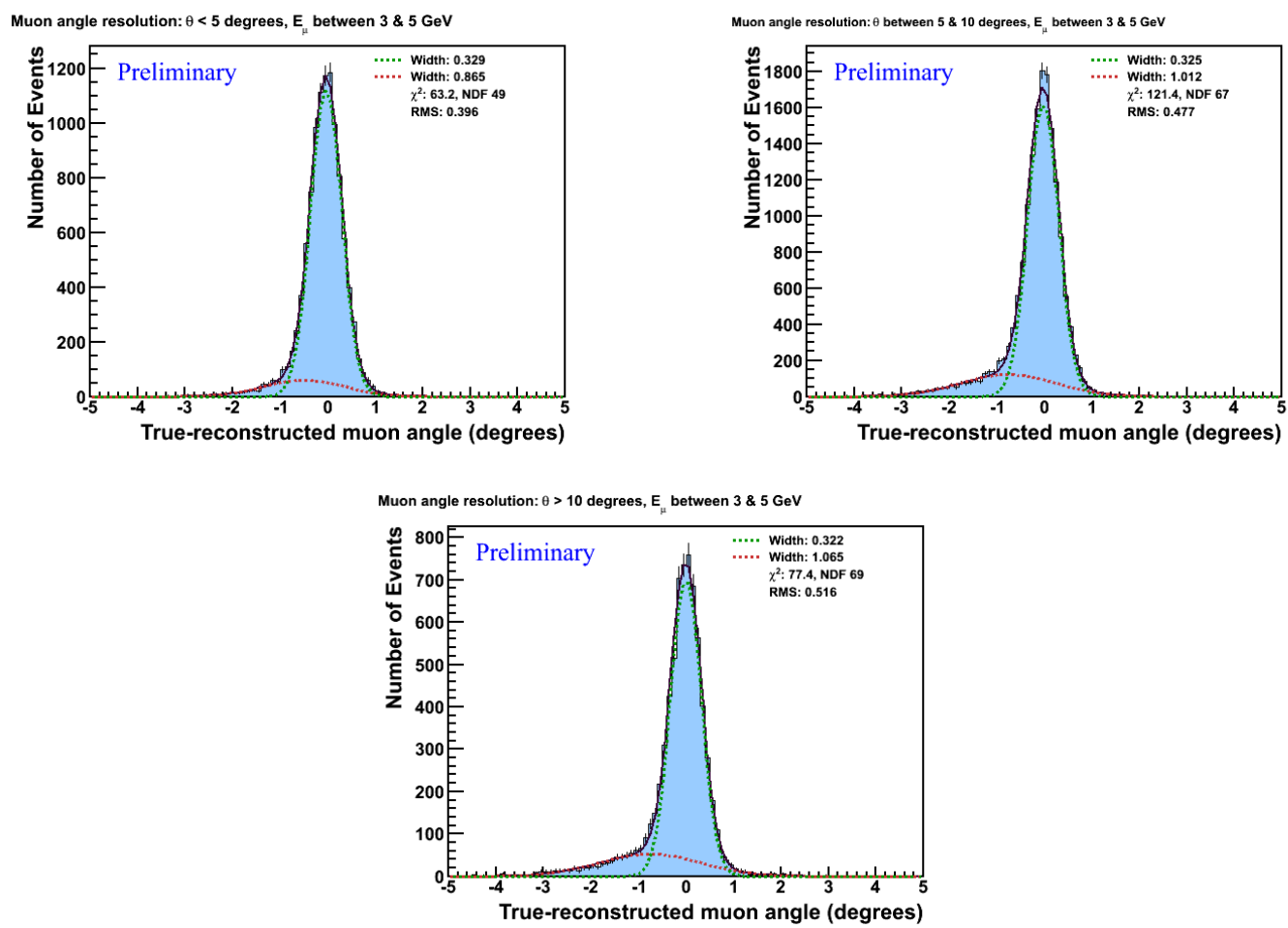

Figure A.16: Muon energy resolution for muons with an energy of 3 to $5 \mathrm{GeV}$. We consider the resolution for different ranges of angle. Plots made by C. Patrick of the MINER $\nu \mathrm{A}$ collaboration. 

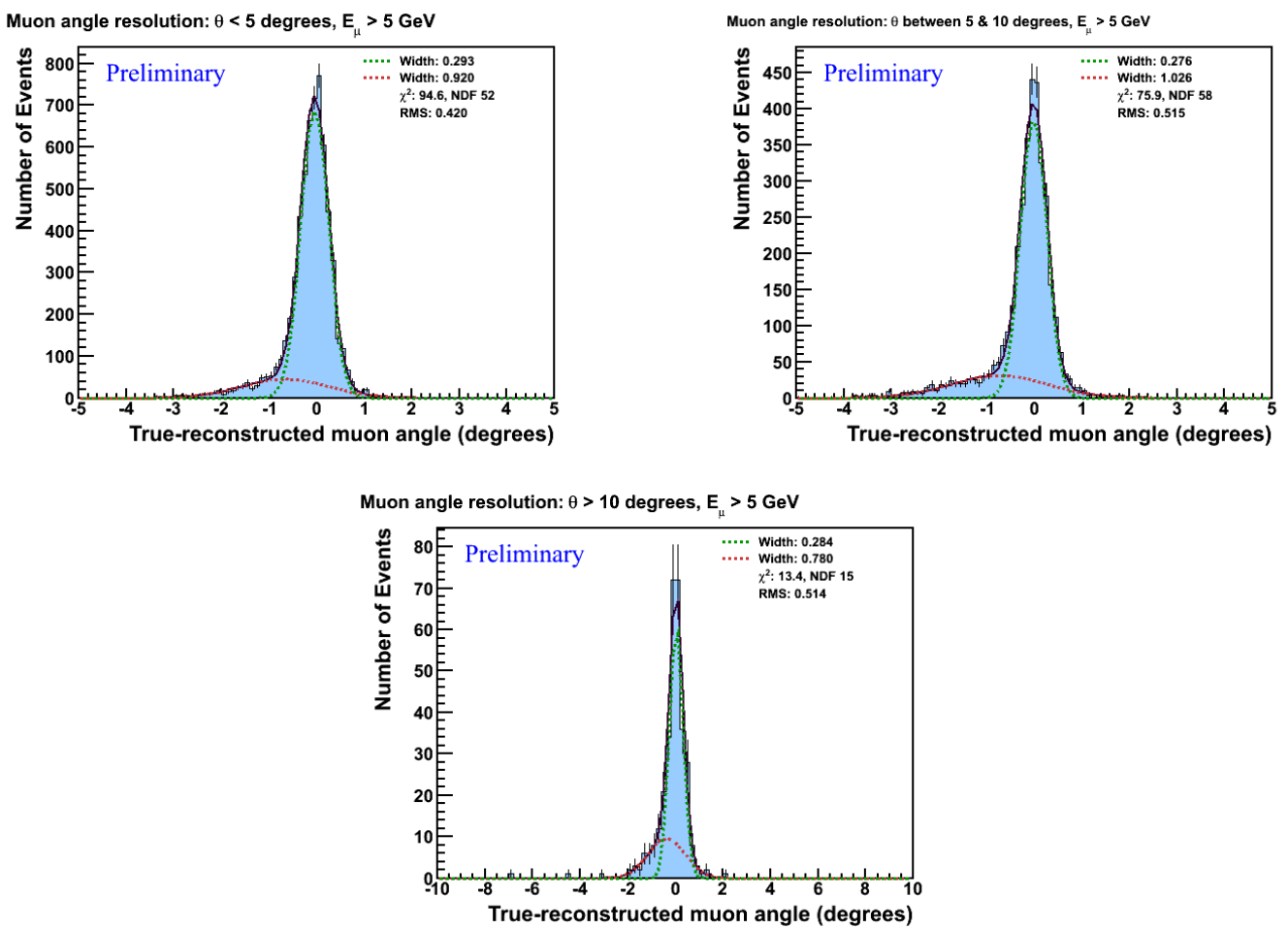

Figure A.17: Muon energy resolution for muons with an energy greater than $5 \mathrm{GeV}$. We consider the resolution for different ranges of angle. Plots made by C. Patrick of the MINER $\nu \mathrm{A}$ collaboration. 

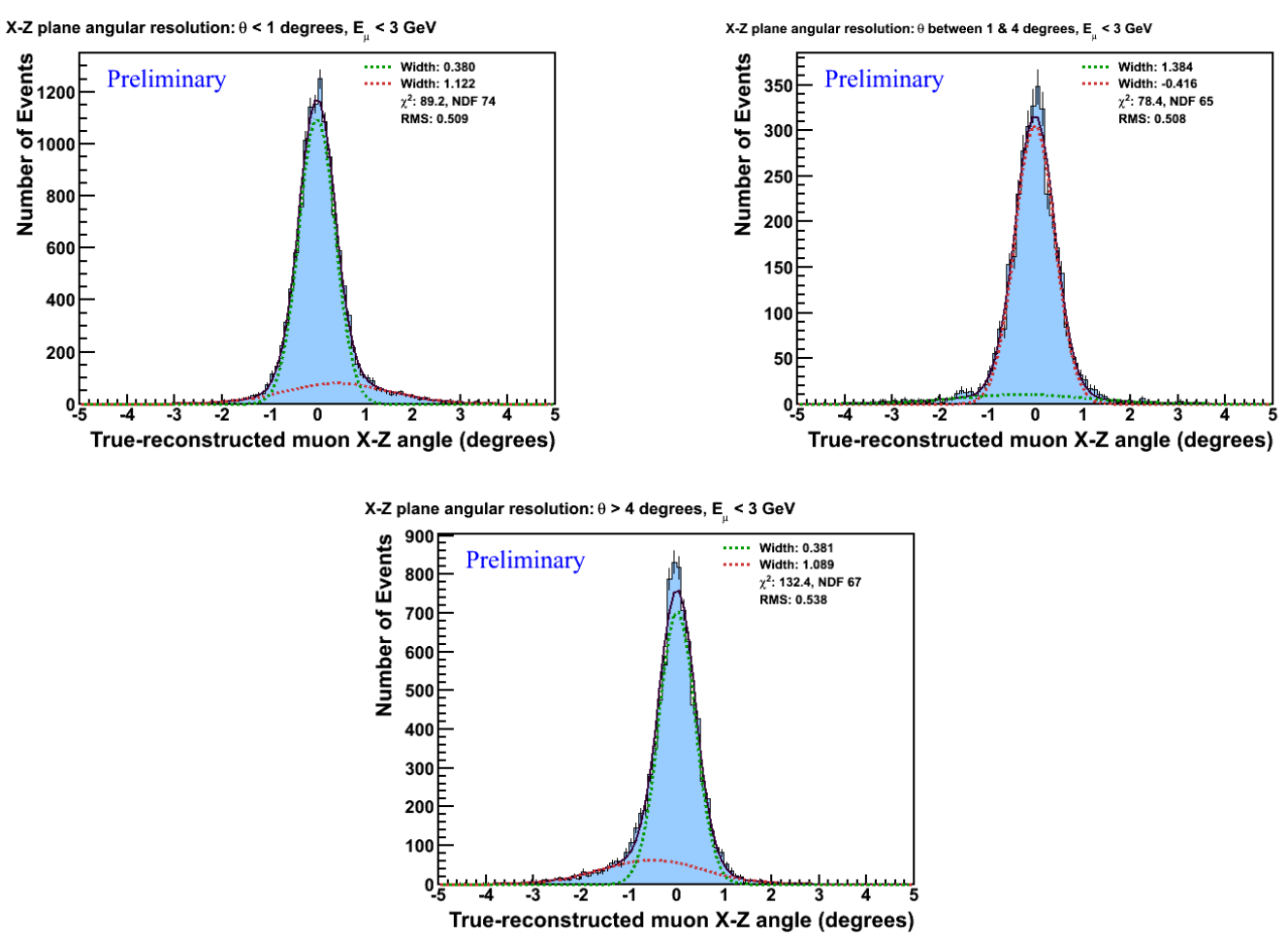

Figure A.18: Muon angle resolution in the XZ plane for muons with an energy of 0 to 3 $\mathrm{GeV}$. We consider the resolution for different ranges of angle. Plots made by C. Patrick of the MINER $\nu \mathrm{A}$ collaboration. 

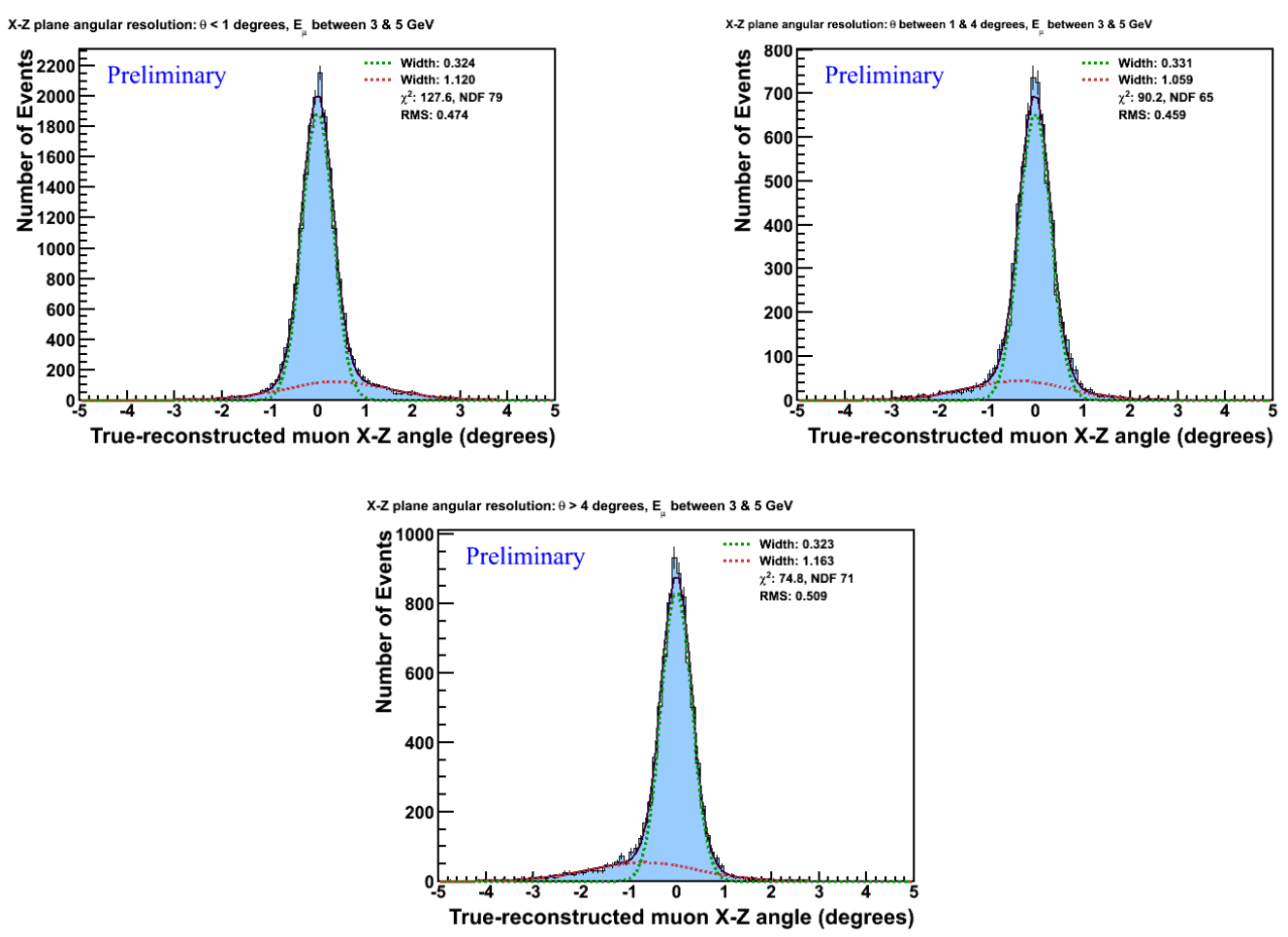

Figure A.19: Muon energy resolution in the XZ plane for muons with an energy of 3 to $5 \mathrm{GeV}$. We consider the resolution for different ranges of angle. Plots made by C. Patrick of the MINER $\nu \mathrm{A}$ collaboration.

\begin{tabular}{c|c|c}
\hline \hline$\theta_{\mu}$ in XZ Plane (degrees) & $\mathrm{E}_{\mu}(\mathrm{GeV})$ & Resolution of $\theta_{\mu}$ in XZ Plane (degrees) \\
\hline$[0,1)$ & {$[0,3)$} & 0.509 \\
{$[1,4)$} & {$[0,3)$} & 0.508 \\
{$[4, \infty)$} & {$[0,3)$} & 0.538 \\
{$[0,1)$} & {$[3,5)$} & 0.474 \\
{$[1,4)$} & {$[3,5)$} & 0.459 \\
{$[4, \infty)$} & {$[3,5)$} & 0.509 \\
{$[0,1)$} & {$[5, \infty)$} & 0.466 \\
{$[1,4)$} & {$[5, \infty)$} & 0.466 \\
{$[4, \infty)$} & {$[5, \infty)$} & 0.513 \\
\hline
\end{tabular}

Table A.4: Muon angle resolution in the XZ plane binned by muon energy and the XZ angle. 

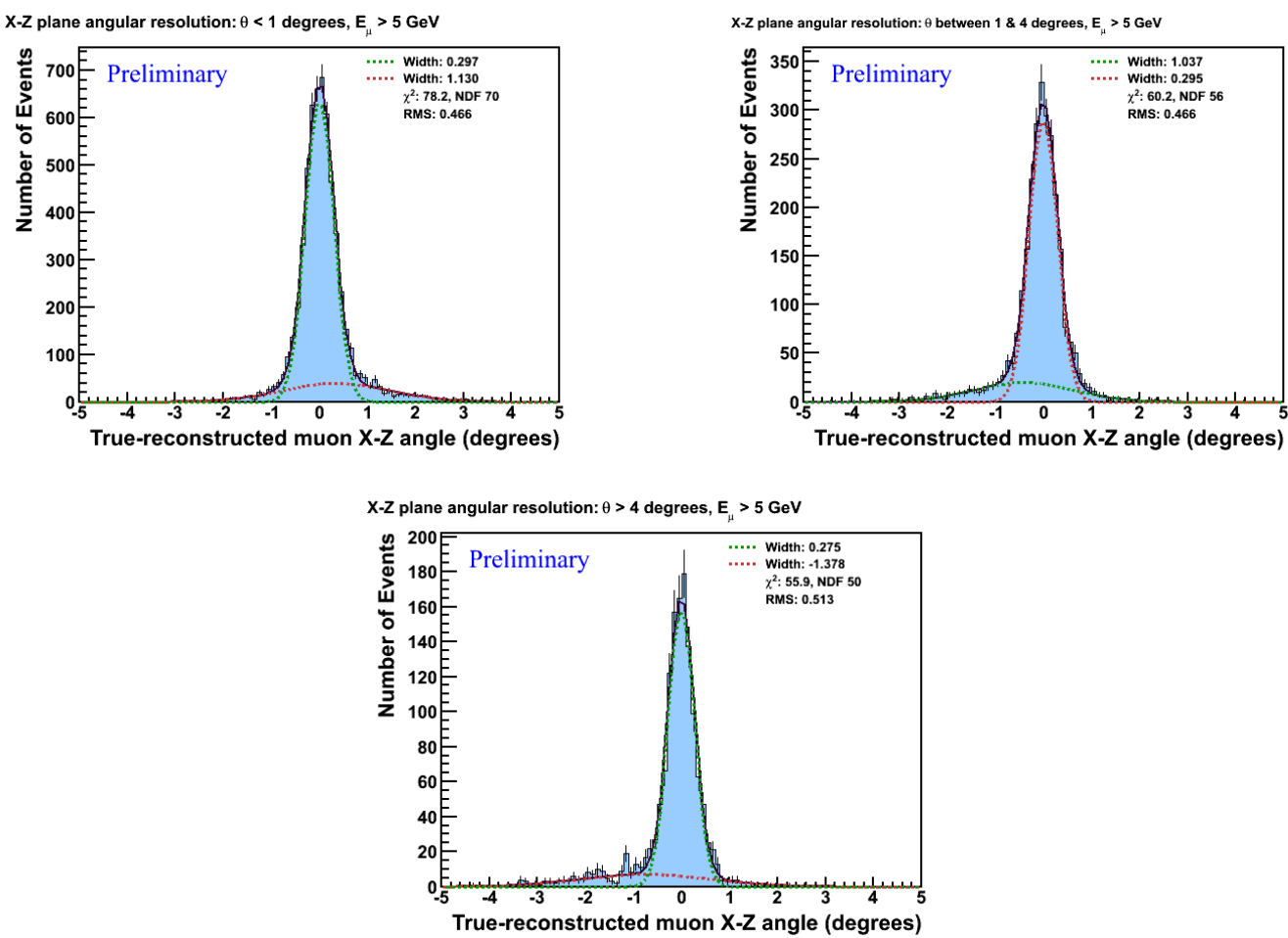

Figure A.20: Muon energy resolution in the XZ plane for muons with an energy greater than $5 \mathrm{GeV}$. We consider the resolution for different ranges of angle. Plots made by $\mathrm{C}$. Patrick of the MINER $\nu$ A collaboration. 

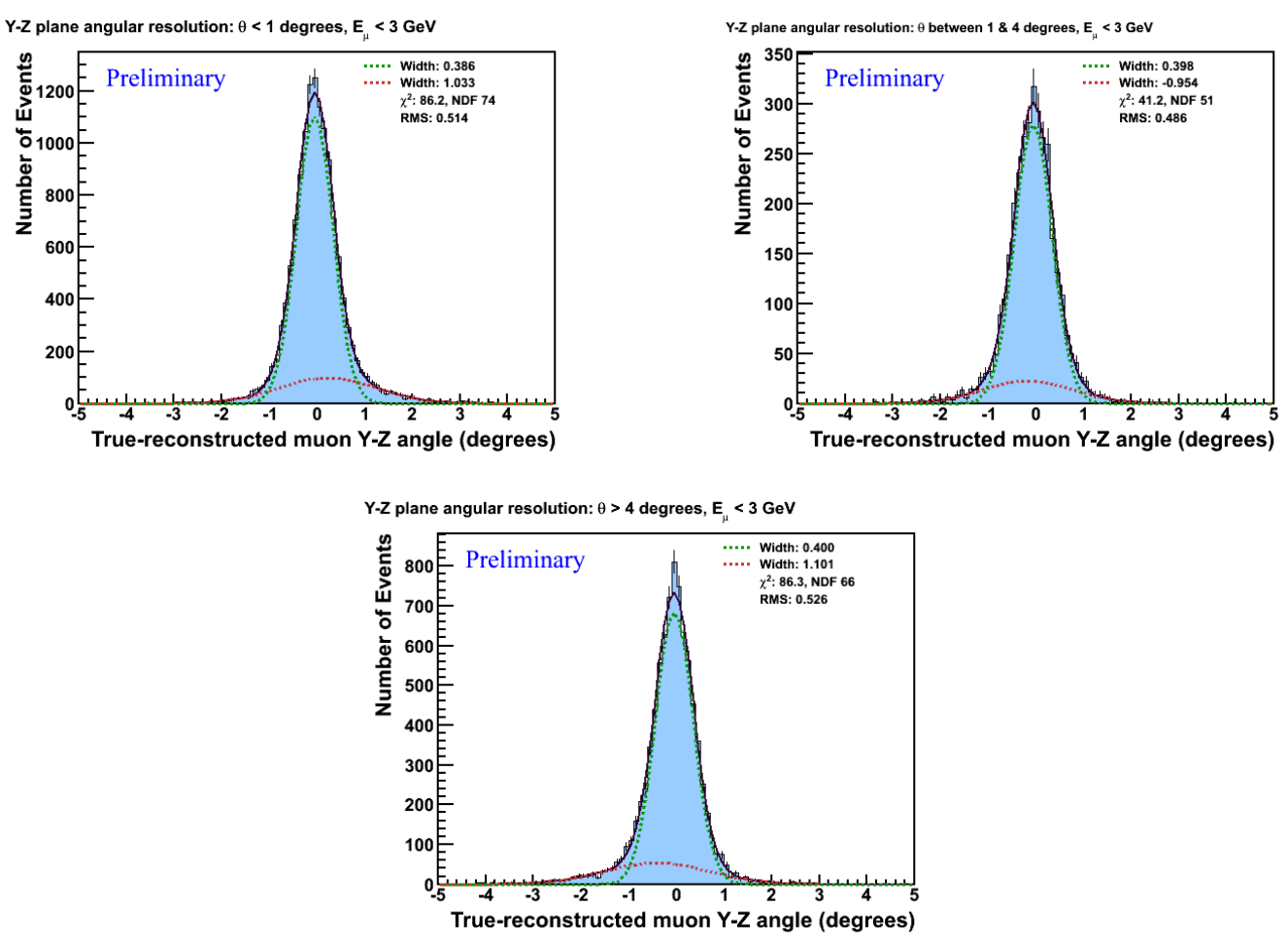

Figure A.21: Muon angle resolution in the YZ plane for muons with an energy of 0 to 3 $\mathrm{GeV}$. We consider the resolution for different ranges of angle. Plots made by C. Patrick of the MINER $\nu \mathrm{A}$ collaboration. 

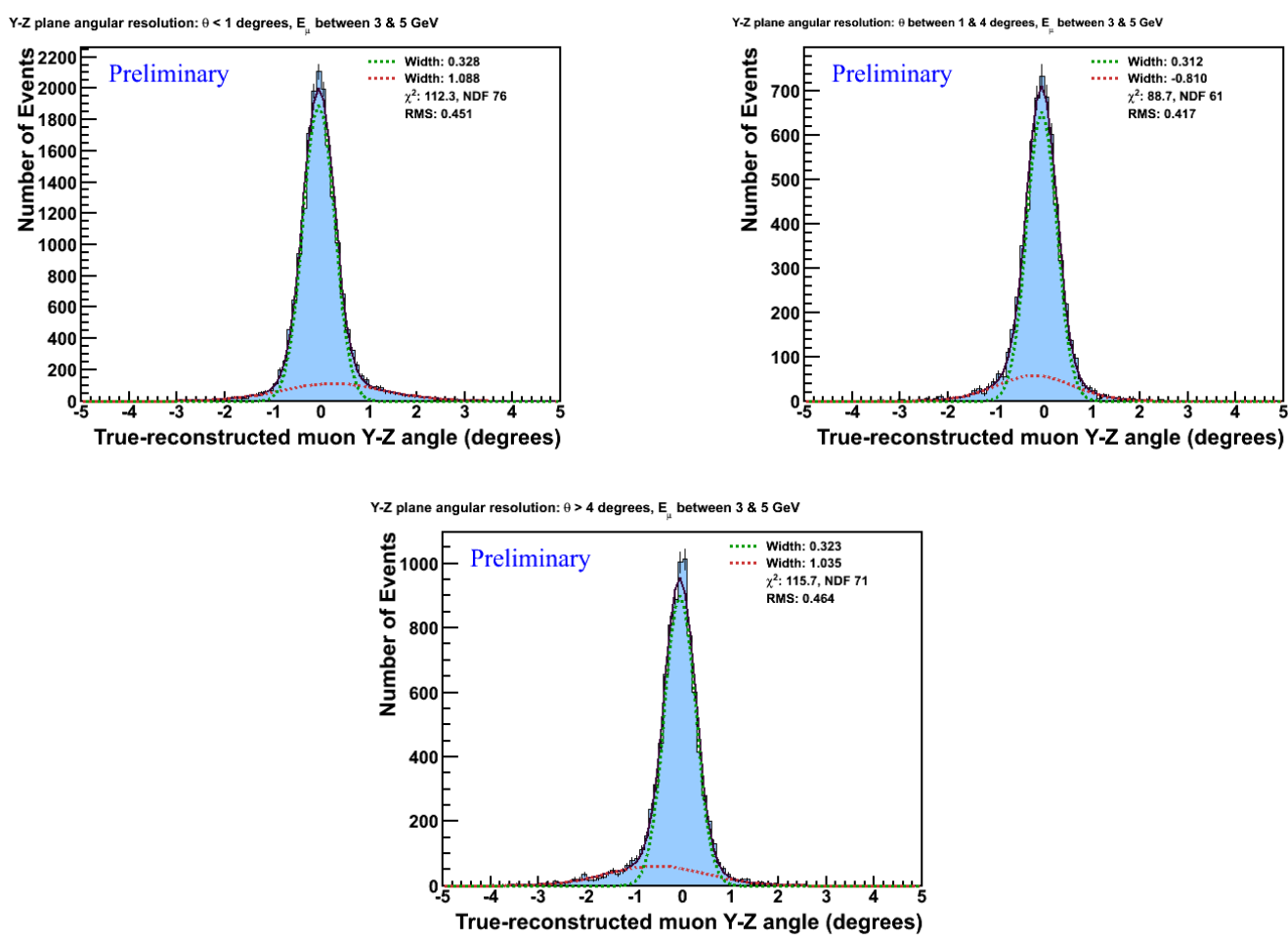

Figure A.22: Muon energy resolution in the YZ plane for muons with an energy of 3 to $5 \mathrm{GeV}$. We consider the resolution for different ranges of angle. Plots made by C. Patrick of the MINER $\nu \mathrm{A}$ collaboration.

\begin{tabular}{c|c|c}
\hline \hline$\theta_{\mu}$ in YZ Plane (degrees) & $\mathrm{E}_{\mu}(\mathrm{GeV})$ & Resolution of $\theta_{\mu}$ in YZ Plane (degrees) \\
\hline$[0,1)$ & {$[0,3)$} & 0.514 \\
{$[1,4)$} & {$[0,3)$} & 0.486 \\
{$[4, \infty)$} & {$[0,3)$} & 0.526 \\
{$[0,1)$} & {$[3,5)$} & 0.451 \\
{$[1,4)$} & {$[3,5)$} & 0.417 \\
{$[4, \infty)$} & {$[3,5)$} & 0.464 \\
{$[0,1)$} & {$[5, \infty)$} & 0.414 \\
{$[1,4)$} & {$[5, \infty)$} & 0.399 \\
{$[4, \infty)$} & {$[5, \infty)$} & 0.473 \\
\hline \hline
\end{tabular}

Table A.5: Muon angle resolution in the YZ plane binned by muon energy and the YZ angle. 

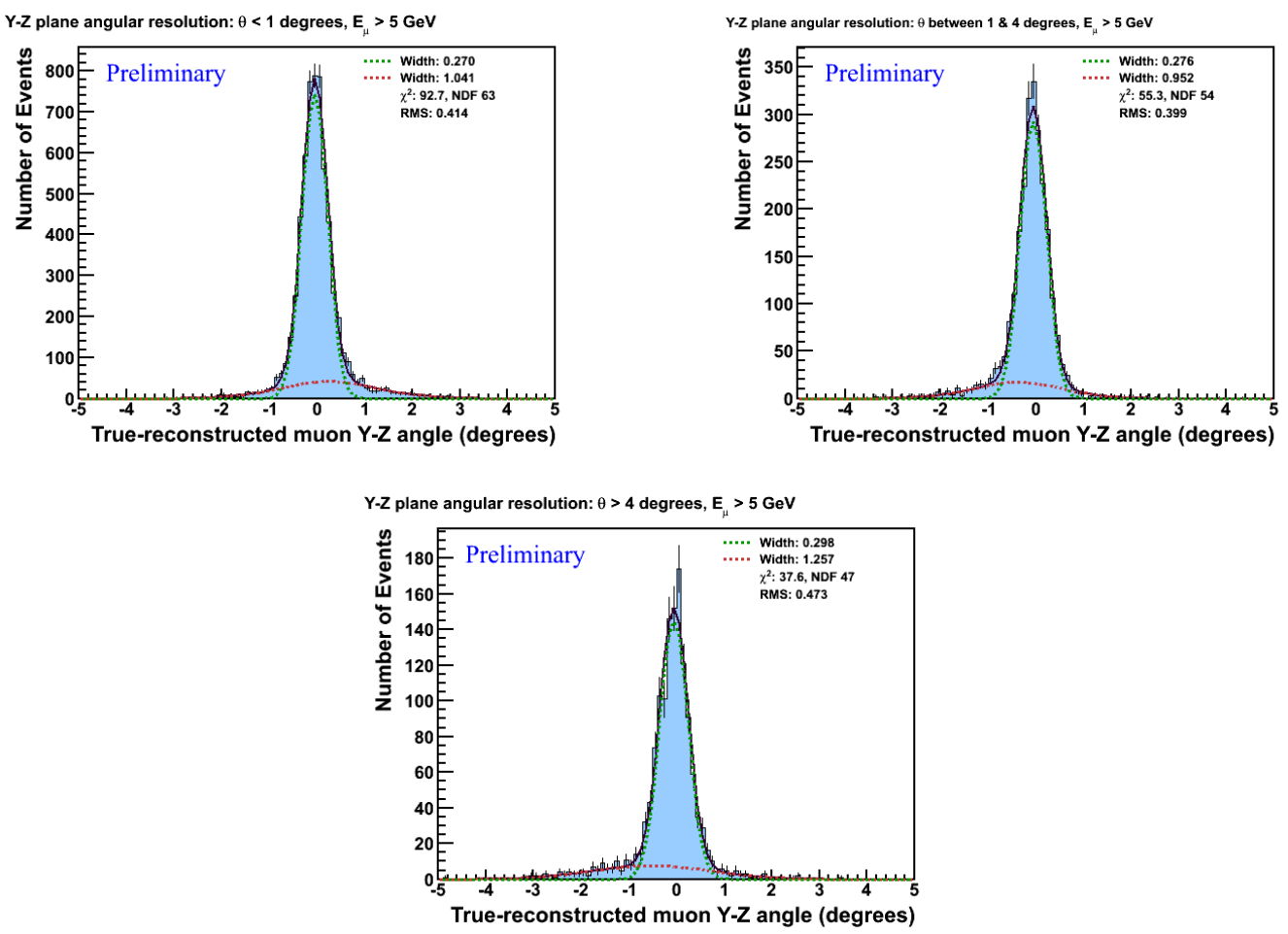

Figure A.23: Muon energy resolution in the YZ plane for muons with an energy greater than $5 \mathrm{GeV}$. We consider the resolution for different ranges of angle. Plots made by $\mathrm{C}$. Patrick of the MINER $\nu \mathrm{A}$ collaboration. 

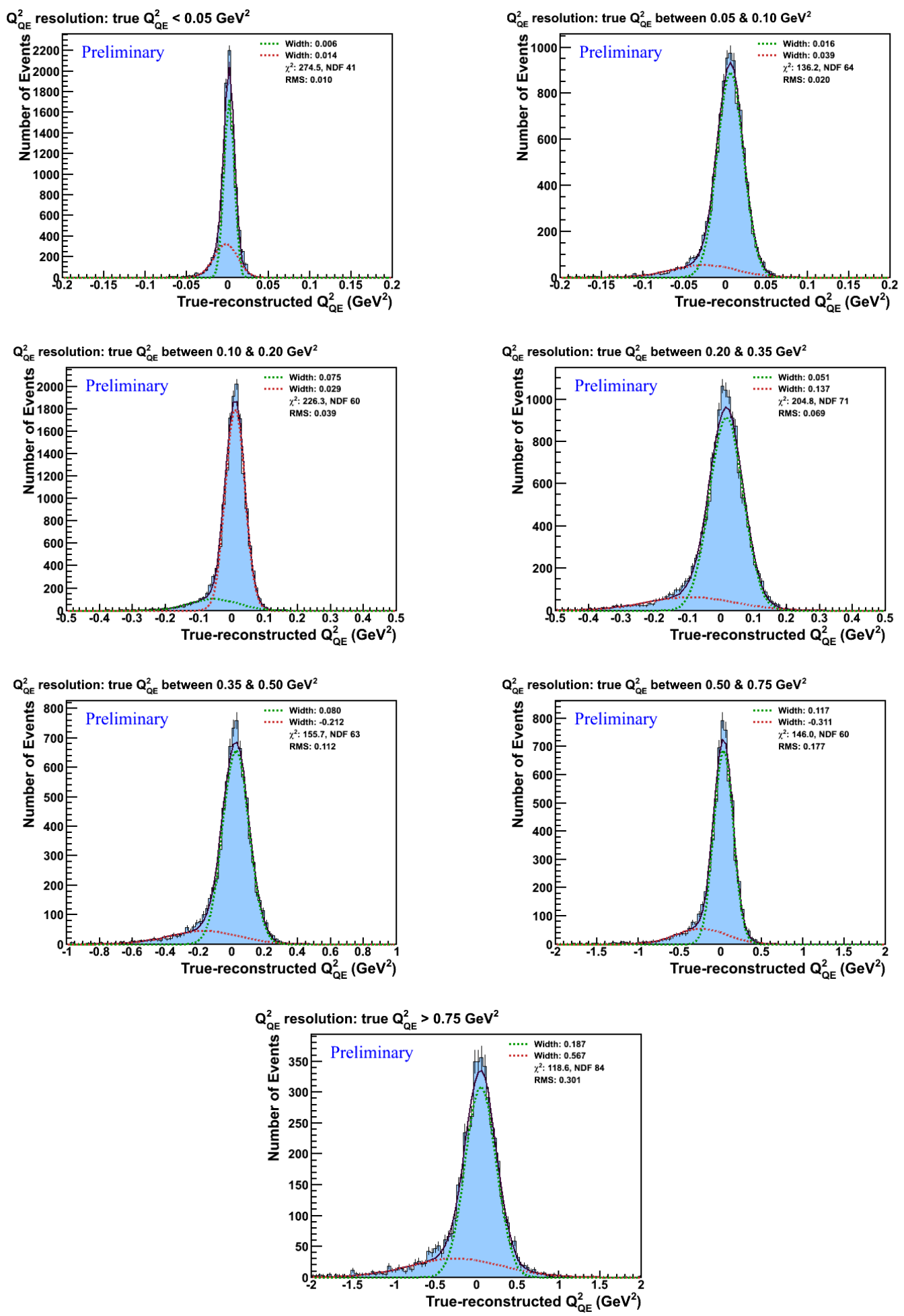

Figure A.24: $\mathrm{Q}_{\mathrm{QE}}^{2}$ resolution plots for CCQE events in the analysis. Plots made by C. Patrick of the MINER $\nu \mathrm{A}$ collaboration. 


\begin{tabular}{c|c}
\hline \hline True $\mathrm{Q}_{\mathrm{QE}}^{2} \operatorname{Bin}\left(\mathrm{GeV}^{2}\right)$ & $\mathrm{Q}_{\mathrm{QE}}^{2}$ Resolution $\left(\mathrm{GeV}^{2}\right)$ \\
\hline$[0,0.05)$ & 0.001 \\
{$[0.05,0.1)$} & 0.020 \\
{$[0.1,0.2)$} & 0.039 \\
{$[0.2,0.35)$} & 0.069 \\
{$[0.35,0.5)$} & 0.112 \\
{$[0.5,75)$} & 0.177 \\
{$[0.75, \infty)$} & 0.301 \\
\hline
\end{tabular}

Table A.6: $\mathrm{Q}_{\mathrm{QE}}^{2}$ resolution, binned by $\mathrm{Q}_{\mathrm{QE}}^{2}$.

\section{$\mathrm{Q}_{\mathrm{QE}}^{2}$ resolution vs $\mathrm{Q}_{\mathrm{QE}}^{2}$}

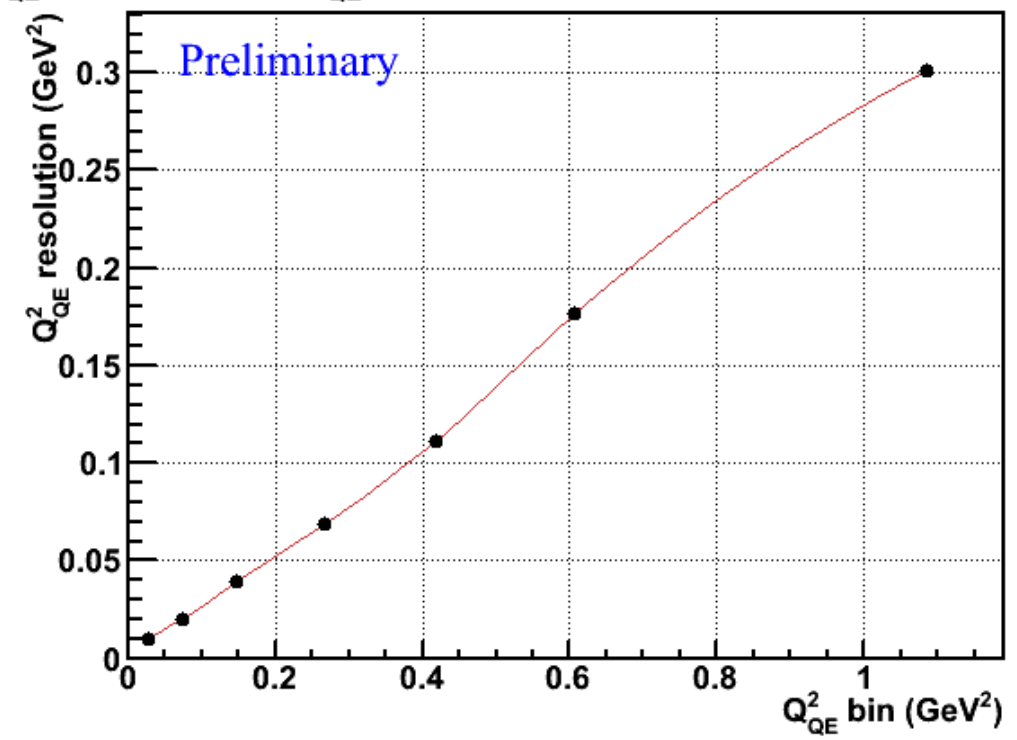

Figure A.25: $\mathrm{Q}_{\mathrm{QE}}^{2}$ resolution as a function $\mathrm{Q}_{\mathrm{QE}}^{2}$. Plot made by C. Patrick of the MINER $\nu \mathrm{A}$ collaboration. 


\section{Appendix B}

\section{The Vertical Slice Test}

The MINER $\nu$ A Vertical Slice Test (VST) was an array of three layers of seven MINER $\nu \mathrm{A}$ inner detector (ID) scintillator strips, visible in Figure B.1. We used cosmic ray muons to study tracking, light yield, and timing resolution in the VST. Additionally, the VST served as a preliminary test for MINER $\nu$ A electronics.

\section{B.1 The VST Aparatus}

The VST attempted to test a variety of systems that would go onto compose the MINER $\nu \mathrm{A}$ detector. What follows is a description of the different components of the test stand and the setup necessary to perform these tests.

\section{B.1.1 Dark Box}

We performed all VST measurements in the VST Dark Box, which was formerly used for quality control of the CMS scintillator barrel panels. To minimize ambient room light interfering with the array, we draped the VST Dark Box with Marvel 

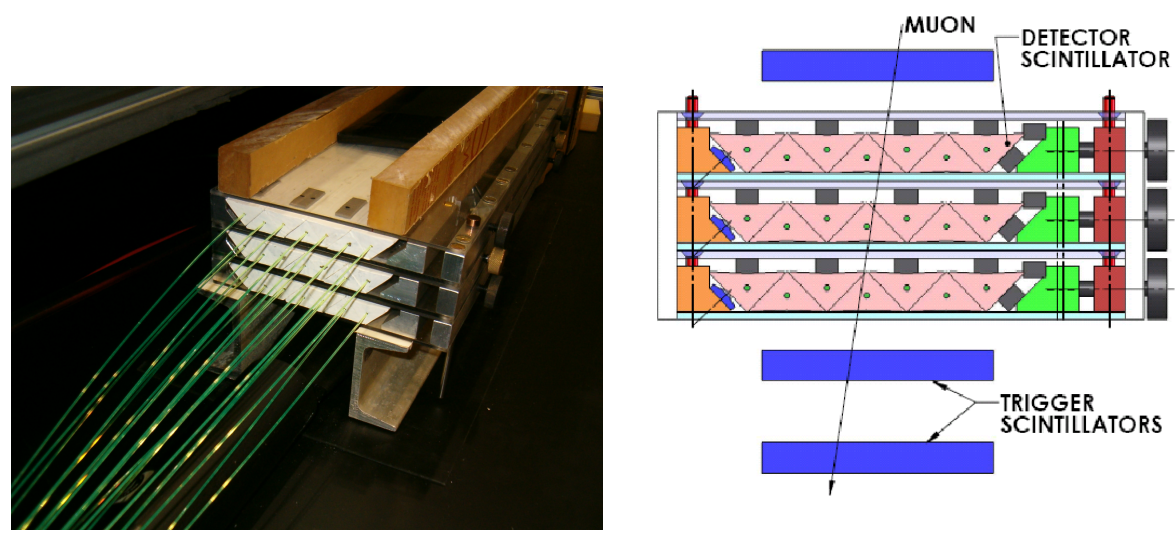

Figure B.1: On the left the Vertical Slide Test detector with both strips and fibers is visible. On the right is a schematic for the detector showing scintillator paddles above and below the array, which are installed to trigger on cosmic ray muons.

Guard and sheets of black polyvinyl fluoride, known as Tedlar [130]. Inside the VST Dark Box, we placed Tedlar on top of and beneath the VST array, the optical fibers, and the box that contained the PMT. We borrowed this box, called a CALDET box, from the MINOS Experiment Test Beam. We established that the VST setup was light tight by collecting data with a single channel PMT in the VST Dark Box under the scenarios:

- The PMT thoroughly wrapped in Tedlar

- The PMT covered with Tedlar in the same way as the VST array, fibers, and CALDET box.

- The PMT without any Tedlar covering.

We found that covering the VST array, fibers, and CALDET box with Tedlar was sufficient to ensure a light tight setup. Without covering it, we observed a noticeable amount of background light. 


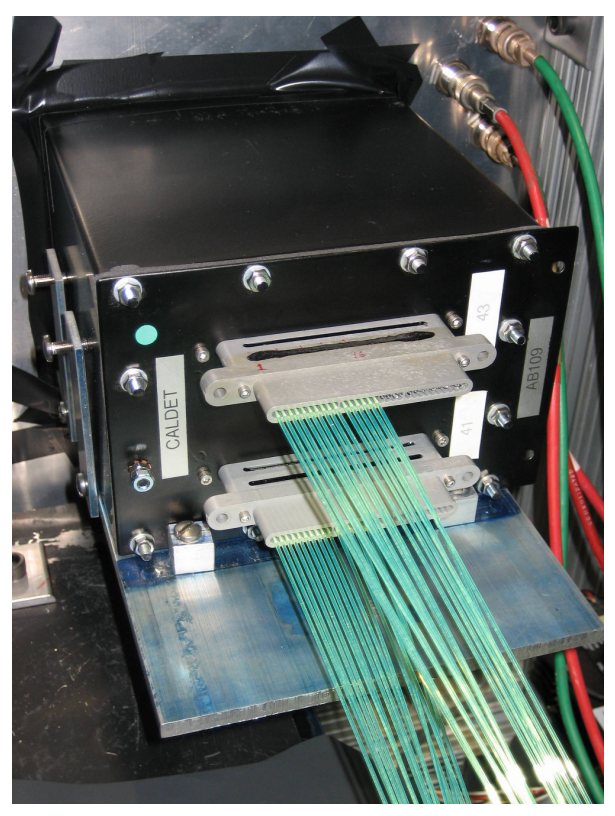

Figure B.2: The CALDET box, which houses the VST PMT, attached to the VST Dark Box. Wavelength shifting fibers are connected to the rear of the CALDET Box.

\section{B.1.2 VST Array}

The VST array was built from 0.5 meter coextruded scintillator strips which are identical to the ones used by MINER $\nu \mathrm{A}$. We discuss the composition and geometry of these strips in Section 3.5.1. We painted both ends of the strips with white EJ-510 $\mathrm{TiO}_{2}$ Eljen paint [131]. A University of Rochester engineer, R. Flight, designed the metal frame that held the VST together.

We used the same WLS optical fibers as MINER $\nu$ A, which we describe in Section 3.5.2, to perform light collection. One end of each fiber had a mirror coating. These ends of the fiber was glued directly into each hole of a scintillator strip. We used 815C TETA epoxy [132] for the gluing. We glued the other end of each fiber into a MINOS connector, which were mated to the CALDET box, shown in Figure B.2. 


\section{B.1.3 VST PMT}

The CALDET box contained a 64-anode R5900 PMT [133]. This PMT should be functionally identical to the ones used in MINER $\nu \mathrm{A}$. The PMT was supplied with $800 \mathrm{~V}$ which corresponds to an average PMT channel gain of $8 \times 10^{5}$. Gains vary greatly from channel to channel. This required us to measure the relative gain for each individual VST channel and include that as a calibration in our analysis of VST data. MINOS also made measurements for individual channel gain for the same PMT which is shown in Table B.1; however, because of the difficulty of mapping from PMT pixels to electronics channels, we used our results only. We discuss this calibration in detail in Section B.2.5. MINOS found the ratio of the channel with highest gain to the channel with the lowest gain to be 2.3 [133]. For channels used in the VST, this ratio was 2.0.

\section{B.1.4 VST Trigger}

The external trigger consisted of three scintillator paddles. One paddle was directly above the array, one directly below the array, and one about half a meter below the other paddle. The paddles directly above and below the array had $8.5 \mathrm{~cm}$ by 18.6 cm overlap with each other. This overlap was centered over the $0.5 \mathrm{~m}$ long VST array. The third paddle was centered beneath the overlapping region of the top two paddles. We did this to ensure that all the muons that trigger the array would have a trajectory close to perpendicular to the surface of the array. The dimensions of the three scintillator paddles were as follows. The scintillator in the top two paddles was $8.5 \mathrm{~cm}$ in width and $24.5 \mathrm{~cm}$ in length. The scintillator in the bottom paddle was $18.2 \mathrm{~cm}$ in width and $25.2 \mathrm{~cm}$ in length. The electronics setup for the triggering 


\begin{tabular}{c|c|c|c|c|c|c|c|c}
\hline \hline Pixels & 1 & 2 & 3 & 4 & 5 & 6 & 7 & 8 \\
\hline 1 & 0.56 & 0.83 & 0.78 & 0.75 & 0.86 & 0.79 & 0.86 & 0.61 \\
\hline 2 & 0.59 & 0.77 & 0.78 & 0.77 & 0.78 & 0.72 & 0.76 & 0.61 \\
\hline 3 & 0.60 & 0.81 & 0.84 & 0.80 & 0.83 & 0.80 & 0.78 & 0.61 \\
\hline 4 & 0.59 & 0.85 & 0.79 & 0.81 & 0.80 & 0.78 & 0.79 & 0.56 \\
\hline 5 & 0.57 & 0.89 & 0.85 & 0.79 & 0.82 & 0.81 & 0.90 & 0.56 \\
\hline 6 & 0.59 & 0.98 & 0.99 & 0.95 & 0.92 & 0.92 & 0.89 & 0.55 \\
\hline 7 & 0.54 & 0.89 & 0.97 & 1.03 & 1.00 & 1.00 & 0.93 & 0.53 \\
\hline 8 & 0.53 & 0.94 & 0.92 & 1.23 & 0.86 & 0.95 & 0.89 & 0.54 \\
\hline \hline
\end{tabular}

Table B.1: Gains of the VST PMT at a voltage of $-798 \mathrm{~V}$ in units of $10^{6}$ for all 64 channels as found by MINOS [133].

is discussed in Section B.1.5.

\section{B.1.5 VST Electronics}

The VST electronics were composed of the following components: the trigger, the DAQ, and the power supplies. We used Model 1880B Dual Channel BCD scalers to monitor cosmic ray muon hits on the trigger paddles. The trigger logic was in a NIM crate mounted in a rack. We used a LRS Model 620BL discriminator to determine if there was a hit on a paddle. We fed the discriminator output to a Model $365 \mathrm{AL}$ Logic Unit and set it to look for triple coincidence.

A gate in which a trigger signal could be received was open for a window of 10 ms. A veto was used to ensure that no trigger could be received outside of the gate live time. A DG2020A pulse generator [134] created the gate and the gate veto. We also used the DG2020A to make pulses for single photoelectron (p.e.) studies discussed in Section B.2.5.

Four Front End Boards (FEBs), shown in Figure B.3, were specially designed so MINER $\nu \mathrm{A}$ electronics components could be used with the CALDET box. The FEBs were prototypes for the eventual FEBs used in MINER $\nu \mathrm{A}$. We installed the 


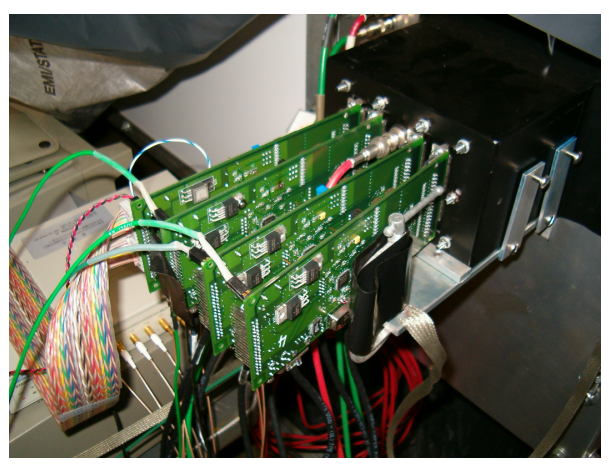

Figure B.3: The four FEBs plugged into the CALDET box. The rainbow colored cable is the parallel port which transmitted data from the FEBs to the computer running the DAQ system

four FEBs into the four card slots on the CALDET box.

The four FEBs communicated through a LVDS chain and had a master-slave arrangement controlled by a fully programmable gate array (FPGA). The master FEB controlled how and when all the FEBs would read and write FPGA frames. A frame is a series of bits sent over the LVDS chain bit by bit and can carry instructions or data. The FPGA controls values such as the gate width and charge integration time along with the master-slave relationship. We numbered the FEBs 5, 6, 7, and 8.

Each FEB contained a 32 channel TriP chip originally designed for D-Zero detector electronics [135]. A schematic of the TriP chip is shown in Figure B.4. Each TriP chip supported 16 high gain channels and 16 low gain channels. A divider located before the TriP chip split the charge from a PMT channel so that approximately $\frac{8}{9}$ of the original charge went to the high gain channel and $\frac{1}{9}$ of the original charge went to the low gain, but small variations existed from channel to channel depending upon the exact values of the resistors and capacitors used to divide the charge. This ratio is discussed in Section B.2.6. Each channel in the TriP chip then 


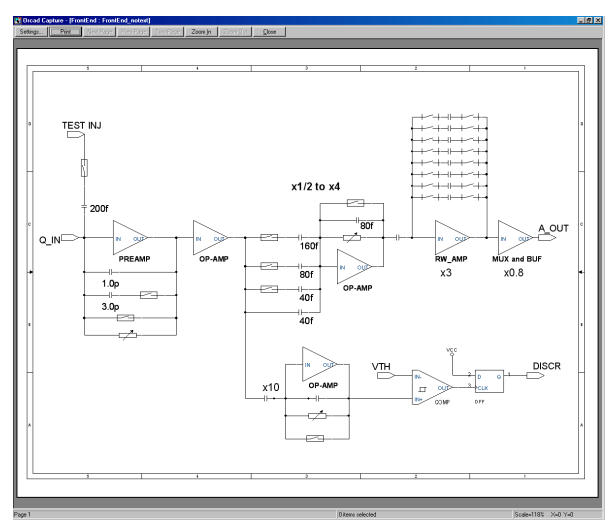

Figure B.4: The schematic for the TriP chip [135]. Gains were adjusted by changing which set of parallel capacitors were used.

went to a 10-bit ADC (maximum 1023 ADC counts) where charge was converted into a number of ADC counts.

Data was read out to the DAQ system through a parallel port cable from the master FEB (FEB 5). The CALDET box was powered by a high voltage Model 1570 calibrated DC power source set to $-800 \mathrm{~V}$. The trigger paddles used the same model of power supply, but used a Phototube High Voltage Zener Divider to set the voltage for each paddle individually. The trigger paddles had voltages set to $-1300 \mathrm{~V}$. The voltages for the paddles were then tuned until triple coincidence could be observed between all three paddles. We found the rate of a spurious triple coincidence to be in the 1 in $10^{6}$ range. Finally, the FEBs were supplied with $5 \mathrm{~V}$ by a LPS DC tracking power supply. The ground for this power supply was connected to the CALDET box and the VST Dark Box.

\section{B.1.6 VST Data Acquisition}

We ran the DAQ with an executable written with Visual Basic [136] on a PC running Windows XP [137]. Using the executable, we could write registers for both FPGA 
chips and TriP chips. We determined settings for the discriminator thresholds and gains to be set on the TriP chips. These values were then set from DAQ program. The DAQ program wrote out the data to an ASCII file which we analyzed using ROOT [94].

The DAQ ran in two different modes, Pedestal Mode (Ped-Mode) and Discriminator Mode. In Ped-Mode, we could read data out with just an external trigger. Discriminator Mode required both an external trigger and ADC counts on at lesat one channel to pass the discriminator threshold set in the TriP chip. TriP chip values for running in both modes are shown in Table B.2. Differences between the $\mathrm{VST}$ and the MINER $\nu \mathrm{A}$ detector

The VST differs from MINER $\nu \mathrm{A}$ in several significant ways. First, the PMT we used in the VST had a quantum efficiency $8.6 \%$ lower than what we expect an average MINER $\nu$ A PMT to have. Second, the VST connectors containing the WLS fibers plugged directly into the CALDET box. The MINER $\nu$ A detector has a clear fiber cable between the WLS fiber and the PMT box. The MINER $\nu$ A clear fiber cables were measured to have an attenuation length of $7.5 \mathrm{~m}$, a longer attenuation length than MINER $\nu$ A WLS fiber. Third, VST WLS fibers were $3.5 \mathrm{~m}$ in length instead of the maximum estimated fiber length of $3.2 \mathrm{~m}$ for MINER $\nu$ A. Considering these differences, we expected MINER $\nu$ A to have a slightly higher light yield.

Lastly, MINER $\nu \mathrm{A}$ and VST electronics differed in a few ways. MINER $\nu \mathrm{A}$ has three ranges of ADC for each PMT channel instead of the two used in the VST. Additionally, MINER $\nu$ A electronics uses a 12-bit ADC. 


\begin{tabular}{c|c|c|c}
\hline \hline Register & Trip Register & Pedestal Mode & Discriminator Mode \\
\hline \hline 1 & IBP & 130 & 130 \\
\hline 2 & IBBNFoll & 120 & 120 \\
\hline 3 & IFF & 0 & 66 \\
\hline 4 & IBPPIFF1REF & 160 & 160 \\
\hline 5 & IBPOPAMP & 24 & 24 \\
\hline 6 & IBPF0l2 & 24 & 24 \\
\hline 7 & IFFP2 & 0 & 42 \\
\hline 8 & VTH & 0 & FEB specific \\
\hline 9 & VREF & 170 & 160 \\
\hline 10 & Gain & 11 & 7 \\
\hline 11 & Pipe Delay & 6 & 3 \\
\hline 12 & IRSEL & 3 & 3 \\
\hline 13 & IWSEL & 3 & 0 \\
\hline 14 & Inject (B3) & 0 & 0 \\
\hline 15 & Inject (B2) & 0 & 0 \\
\hline 15 & Inject (B1) & 0 & 0 \\
\hline 15 & Inject (B0) & 0 &
\end{tabular}

Table B.2: TriP chip register values for Ped-Mode and Discriminator Mode. The VTH settings for Discriminator Mode, which controls the discriminator threshold for each FEB, were tuned for each FEB. The FEBs had the following VTH settings for discriminator mode: FEB $5=227$, FEB $6=225$, FEB $7=228$, and FEB $8=$ 232 .

\section{B.1.7 Scaling the VST to MINER $\nu \mathrm{A}$}

Due to the differences between the $\mathrm{VST}$ and MINER $\nu \mathrm{A}$, results for the two will differ slightly. To scale results between the two, we calculated the relative light yield $\mathrm{L}_{\text {relative }}$ for each. The differences between the VST and MINER $\nu \mathrm{A}$ are listed in Table B.3. Our formula for $\mathrm{L}_{\text {relative }}$ is shown in Equation B.1. We use the ratio of both $\mathrm{L}_{\text {relative }}$ to scale the light yield measured with the VST to what we expect in MINER $\nu \mathrm{A}$. We expect the average light yield in MINER $\nu \mathrm{A}$ to be 1.093 more than the light yield of the VST.

$$
\mathrm{L}_{\text {relative }}=\mathrm{QE} * \mathrm{~F}_{\mathrm{c}} * \mathrm{~F}_{\mathrm{gl}} * \mathrm{~F}_{\text {mirror }} * \mathrm{~F}_{\mathrm{d}} * \mathrm{~F}_{\mathrm{acc}} * \mathrm{~F}_{\mathrm{gr}} * \mathrm{e}^{\left(\frac{\mathrm{f}_{\mathrm{l}}-\mathrm{fmeasured}}{\mathrm{f}_{\text {att }}}\right)}
$$




\begin{tabular}{c|c|c}
\hline \hline Differences in Configuration & VST & MINER $\nu \mathrm{A}$ \\
\hline \hline Point Along Fiber where Measurement is Made & $25 \mathrm{~cm}$ & $0 \mathrm{~cm}$ \\
\hline WLS Fiber Length & $350 \mathrm{~cm}$ & $320 \mathrm{~cm}$ \\
\hline Connector Transmission & 0.85 & 0.6 \\
\hline Grease Connection & 1 & 1.35 \\
\hline Effective Cable Attenuation & 1.00 & 0.81 \\
\hline Mirroring Factor & 1.72 & 1.80 \\
\hline Effective Photosensor Quantum Efficiency & 0.116 & 0.127 \\
\hline Relative Light Yield & 0.089 & 0.097 \\
\hline \hline
\end{tabular}

Table B.3: A list of the differences between the VST and MINER $\nu$ A including the ratio of the relative light yields.

In Equation B.1, QE is quantum efficiency, $f_{l}$ fiber length, $f_{\text {measured }}$ the point along the fiber where the measurement is taking place, $f_{\text {att }}$ the attenuation length of the fiber, $\mathrm{F}_{\mathrm{c}}$ the fraction of light transmitted from WLS fiber to the PMT, $\mathrm{F}_{\mathrm{gl}}$ a factor from the type of epoxy used to glue the fiber, $\mathrm{F}_{\text {mirror }}$ a factor accounting for how the fiber was mirrored, $\mathrm{F}_{\mathrm{D}}$ a factor accounting for fiber diameter, $\mathrm{F}_{\mathrm{acc}}$ from PMT pixel acceptance, and $\mathrm{F}_{\mathrm{gr}}$ a factor from greasing the fiber connections.

\section{B.2 Performing Tests with the VST}

What follows is a description of how measurements were made with the VST.

\section{B.2.1 Running the VST}

To understand results from the VST, a variety of studies had to be performed. This included the following:

- Finding the mapping between VST strips and electronics channels

- Finding the charge to ADC count relationship 


\begin{tabular}{c|c|c|c|c|c|c|c}
\hline \hline & Strip 1 & Strip 2 & Strip 3 & Strip 4 & Strip 5 & Strip 6 & Strip 7 \\
\hline \hline Layer 1 & B5C13 & B5C4 & B5C7 & B6C5 & B7C8 & B7C11 & B7C2 \\
\hline Layer 2 & B8C9 & B8C3 & B5C12 & B5C8 & B5C11 & B5C2 & B7C12 \\
\hline Layer 3 & B7C16 & B7C6 & B8C13 & B8C4 & B8C7 & B5C5 & B6C10 \\
\hline \hline
\end{tabular}

Table B.4: The mapping of VST strips to electronics channels.

- Finding and understanding the pedestals for each channel

- Finding the single p.e. peak for each channel

- Finding the low gain to high gain ADC ratio for each channel

- Finding the optimum discriminator threshold for each board

\section{B.2.2 Mapping the Array}

Since the CALDET box has a complicated mapping of PMT pixels to electronics channels, we performed mapping using an LED. To determine the mapping, we put a pulsing LED over each fiber individually and observed which electronics channel had a signal. The mapping for the VST array is shown in Table B.4.

\section{B.2.3 Charge to ADC Ratio and TriP Chip Gain}

The charge to ADC ratio is necessary for determining the proper setting for TriP chip gain. For a N-bit ADC, a maximum ADC value of $2^{\mathrm{N}-1}$ is possible. For a 10 -bit ADC, the maximum value is 1023, however; the VST electronics experienced saturation before 1023 counts. Consequently, we had to determine the charge to ADC ratio and the value at which the ADC began experiencing measurable saturation.

We tested each channel at a fixed charge and gain. To do this, we constructed a Charge Injection Circuit (CIC) so that for every $1 \mathrm{mV}$ of input into the circuit, a 


\begin{tabular}{c|c}
\hline \hline FEB & fC/ADC \\
\hline \hline 5 & 1.894 \\
\hline 6 & 1.848 \\
\hline 7 & 1.873 \\
\hline 8 & 1.712 \\
\hline \hline Avg & 1.832 \\
\hline \hline
\end{tabular}

Table B.5: The charge to ADC ratio for select VST FEB channels.

charge of $1 \mathrm{fC}$ was created as output. We also checked each channel with the CIC to verify that all gains were within $20 \%$ of each other.

We chose TriP chip gains such that we had sensitivity to single p.e.'s and good linearity over the range of charges observed in the VST. These gain settings are bit settings that determine which capacitors are used in the TriP chip. By choosing different combinations of capacitors, the gain can vary greatly. We found that a TriP gain settings of 11 , roughly $1.8 \mathrm{fC} / \mathrm{ADC}$, was optimum.

We mapped out the charge to ADC ratio on one channel for each FEB. Our results are in Table B.5. We only tested one channel per board since these studies were very time consuming and an automated method was not available. We assumed that every channel had a charge to ADC ratio of $2 \mathrm{fC} / \mathrm{ADC}$ count. Charge enters our analysis only for a modest charge slewing correction term explained in Section B.3.4. As a result, the impact of this assumption was small.

\section{B.2.4 Pedestal Measurements}

We studied FEB pedestals and pedestal behavior for all 64 electronics channels. All pedestals were taken with the DAQ in Ped-Mode. An example of high and low gain pedestals for a channel is shown in Figure B.5. We found that high gain pedestals were roughly twice as wide as low gain pedestals in terms of ADC counts. 

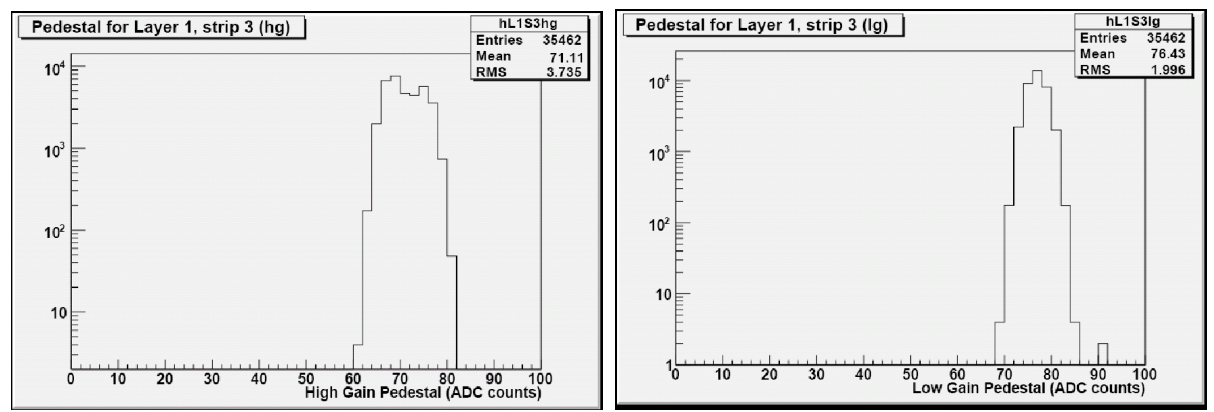

Figure B.5: A plot of the high gain and low gain pedestal for a channel.

We observed that pedestal RMS ranged from 1.5 to $2.5 \mathrm{ADC}$ counts for low gain channels and about 3.5 to 4.5 ADC counts for high gain channels. Since there are an average of $1.83 \mathrm{fC} / \mathrm{ADC}$, that corresponds to noise of 6.4 to $8.2 \mathrm{fC}$ for high gain channels and roughly 22 to $37 \mathrm{fC}$ for low gain channels.

After finding the pedestal mean for each channel, we examined whether outlying ADC counts were distorting the pedestal mean and RMS. We used an iterative method we called the Iterative RMS Cut to ensure that we knew these quantities accurately. First, we calculated the pedestal mean and RMS for each channel. Second, we looked for all values that were within three times the RMS of the pedestal mean. Third, we recalculated the mean and RMS only using values within three times the RMS from the mean.

A total of five iterations of the Iterative RMS Cut were performed. Although pedestal means did not shift much, the high gain and low gain RMS were reduced noticeably. After five iterations, the RMS reached a stable point. Performing five iterations should lead to a truncated RMS that is 0.74 times the true RMS. When the iterations were completed, we scaled the truncated RMS by a factor of $1 / 0.74$ $=1.35$ to get the true RMS. We used the rescaled pedestal mean and RMS in our tracking code instead of using the raw mean and RMS. 
We subtracted the pedestal mean found using the Iterative RMS Cut method for each channel. We use the rescaled high gain RMS to determine if an ADC count during an event is significantly above pedestal.

The last pedestal related phenomenon we examined was common mode noise $(\mathrm{CMN})$. We looked at this for high gain channels only. To perform this study, we made a profile plot of the average of all pedestals for all channels for a single FEB on the $\mathrm{X}$-axis versus the pedestal for a particular channel for that same FEB on the Y-axis. The channel being plotted on the Y-axis was omitted in calculating the average of the pedestals for that FEB. We then applied a linear fit to each plot and recorded the slope from the fit. Examples of this can be seen in Figure B.6.

The average pedestal for a channel generally fell at the midpoint of the fitted line. The average of the board pedestals $\mathrm{P}_{\mathrm{FEBAvg}}$, also tended to fall at this midpoint. We called that point the mean board pedestal average, or $\mathrm{P}_{\mathrm{MBA}}$. The $\mathrm{P}_{\mathrm{MBA}}$ and the slope from the fit were used for a correction term that we added onto the pedestal. The extracted slopes varied from about 0.3 to 1.4. The correction term, C, took the form

$$
\mathrm{C}=\mathrm{s}_{\mathrm{fit}} *\left(\mathrm{P}_{\mathrm{MBA}}-\mathrm{P}_{\mathrm{FEBAvg}}\right),
$$

with $\mathrm{s}_{\mathrm{fit}}$ the fitted value of the slope. The board pedestal average does not include the channel of interest as mentioned before. This correction made some improvement in the RMS. We observed that the larger the pedestal RMS, the greater the improvement from the CMN correction. We observed improvement in the RMS in 15 out of 21 VST channels. The RMS for the other six channels did not show much change.

After performing the above correction, we attempted to optimize the slope to 

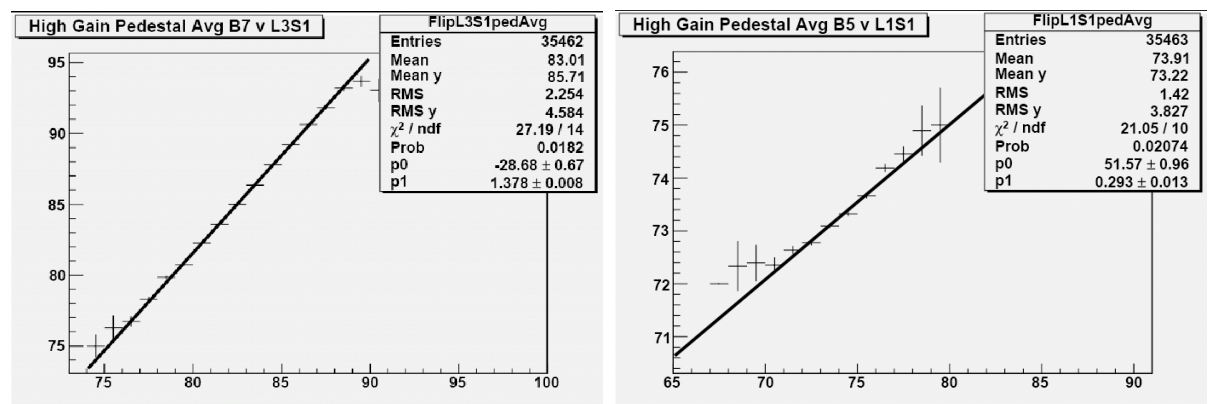

Figure B.6: Two examples of the average of pedestals for a FEB versus channel pedestal. Fit results to a line are also shown.

improve the RMS further. To test this, the slope was varied from 0.0 to 1.5 by increments of 0.1 , the results of which are shown in Figure B.7. We found that the slope from the fit generally gave the optimum correction to CMN.

After we performed the CMN correction, we found the RMS for high gain channels ranged from 3.3 to 3.7 ADC counts. That corresponds to noise of 6.6 to 7.4 $\mathrm{fC}$, an improvement over the 7 to $9 \mathrm{fC}$ mentioned above. Despite this improvement, our pedestals were still noisier than we anticipated. After performing this study, we found the effect small enough to not warrant a correction.

\section{B.2.5 Single Photoelectron Peak}

PMT gains vary greatly from channel to channel; however, the gain for each channel must be known relatively well to have good energy resolution. To map these gains, we made a series of measurements with an LED. First, we bundled all fibers loosely together. We then placed a filtered LED to have complete coverage over these fibers. We used the filter to decrease light by a factor of 1000 to avoid swamping the PMT with light. The DG2020 sent a 4-5 mV pulse to the LED. To find the appropriate voltage, we monitored the PMT dynode on an oscilloscope. The dynode sums all 

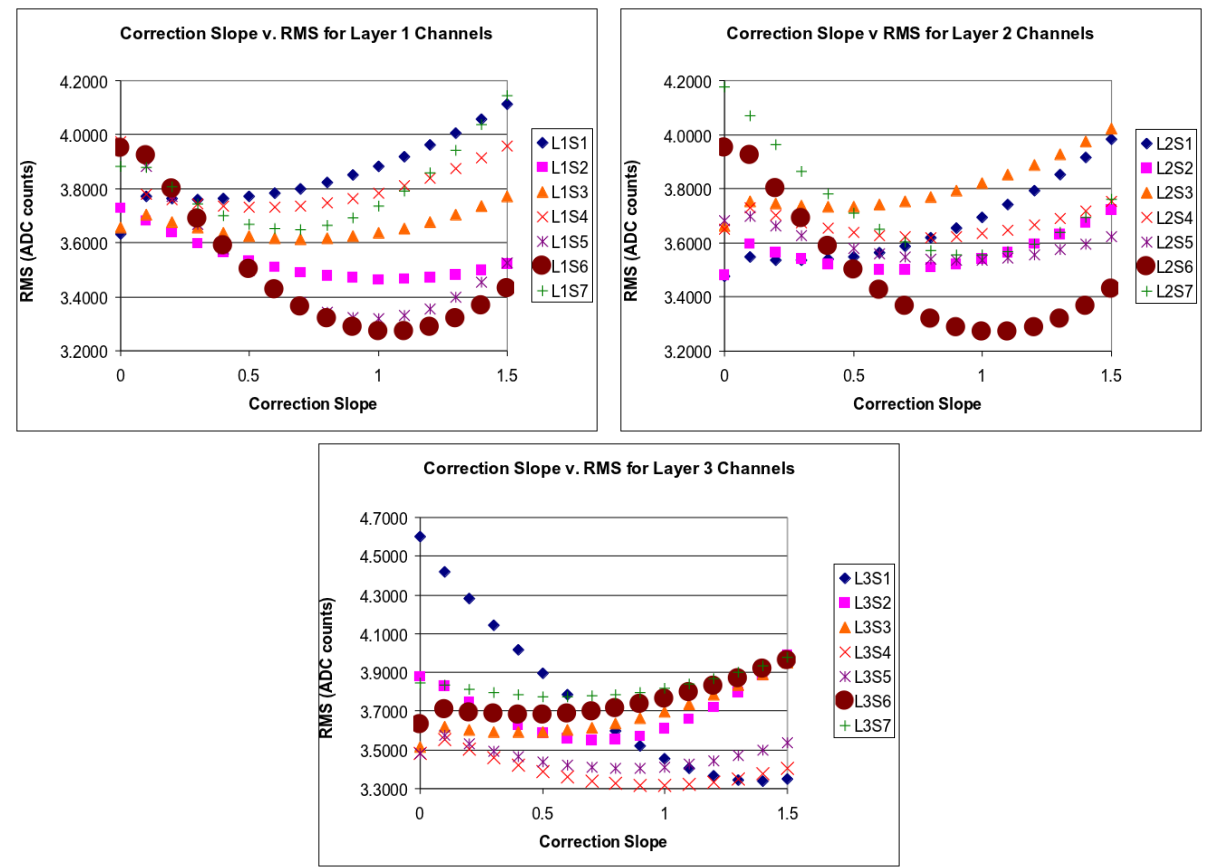

Figure B.7: Fit results by VST layer. The slope was varied from 0.0 to 1.5 to find the optimum value for the fit. We found that the slopes initially found in the fitted CMN plots were close to the optimum value.

charge on all channels of the PMT. We stepped up the voltage for the LED slowly until we saw p.e.'s. We took extreme caution due to the lack of a replacement PMT.

Once we observed single p.e.'s, we took data in Ped-Mode. We performed runs at several different voltages. This was done to get the optimum number of mean p.e.'s for fitting the p.e. distribution. If the mean number of p.e.'s is too small, the statistics will be very poor. If the mean number of p.e.'s is high, we would need many summation terms for our fitting function. A low number of p.e. per trigger means faster convergence of our fitting function. We found the optimum number of p.e.'s for fitting to be around 0.5 p.e. per trigger. For our fitting function, we used 
a poisson distribution with gaussian smearing,

$$
\begin{aligned}
& f\left(\mu, \sigma,<p e>, \mu_{\text {ped }}, \sigma_{\text {ped }}\right)= \\
& \quad N_{\text {events }} *\left(e^{-<p e>} \frac{1}{\sigma_{\text {ped }} \sqrt{2 \pi}} e^{\frac{-\left(x-\mu_{p e d}\right)^{2}}{2 \sigma_{p e d}}} \sum_{n=1}^{N} \frac{<p e>^{n} e^{-<p e>} e^{\frac{\left(x-\mu_{p e d}-n \mu\right)^{2}}{2 \sigma^{2} n}}}{n ! \sigma \sqrt{2 \pi n}}\right),
\end{aligned}
$$

where $\mathrm{N}_{\text {events }}$ is the number of events in the distribution, $\mu_{\text {ped }}$ is the pedestal mean, $\sigma_{\text {ped }}$ is the pedestal RMS, <pe $>$ is the mean number of p.e.'s, $\mu$ is mean of the single p.e. peak, and $\sigma$ is the RMS of the single p.e. peak. The formula sums over $\mathrm{n}$, all the possible p.e. distributions; however, if the value of $<$ pe $>$ is small, this equation will converge quickly. Consequently, we summed to $\mathrm{n}=15$ since including even higher terms has no effect. The $<$ pe $>$ term is the mean number of p.e. per trigger.

We performed the p.e. distribution fit in ROOT. We found the RMS for the single p.e. peak to be very large, around $70-80 \%$ the size of the single p.e. peak mean itself. A table of single p.e. peak values and the corresponding RMS values can be found in Table B.6. Some examples of fits can be found in Figure B.8.

The fit looked best for $<$ pe $>=0.5$ p.e.. Although a variety of runs at different mean number of p.e.'s were performed, all of which looked reasonable, we used the fit with the best statistics for the single p.e. peak in later processing. We found single p.e. peaks for high gain channels only since high and low gain ADC can be scaled to each other. 


\begin{tabular}{c|c|c}
\hline \hline Strip \# & Single PE Peak & Single PE RMS \\
\hline \hline L1S1 & 34.8 & 29.1 \\
\hline L1S2 & 33.0 & 25.1 \\
\hline L1S3 & 50.6 & 40.7 \\
\hline L1S4 & 59.8 & 46.6 \\
\hline L1S5 & 57.7 & 42.1 \\
\hline L1S6 & 50.3 & 38.7 \\
\hline L1S7 & 60.4 & 39.7 \\
\hline L2S1 & 54.0 & 41.2 \\
\hline L2S2 & 31.3 & 24.4 \\
\hline L2S3 & 32.9 & 28.0 \\
\hline L2S4 & 32.4 & 27.9 \\
\hline L2S5 & 44.1 & 40.8 \\
\hline L2S6 & 55.0 & 38.6 \\
\hline L2S7 & 49.4 & 38.2 \\
\hline L3S1 & 46.7 & 37.9 \\
\hline L3S2 & 57.5 & 42.2 \\
\hline L3S3 & 44.0 & 38.0 \\
\hline L3S4 & 55.5 & 38.3 \\
\hline L3S5 & 33.5 & 25.8 \\
\hline L3S6 & 30.2 & 27.6 \\
\hline L3S7 & 47.9 & 38.8 \\
\hline \hline
\end{tabular}

Table B.6: Single p.e. mean and single p.e. RMS values for all channels of the VST in units of ADC counts.

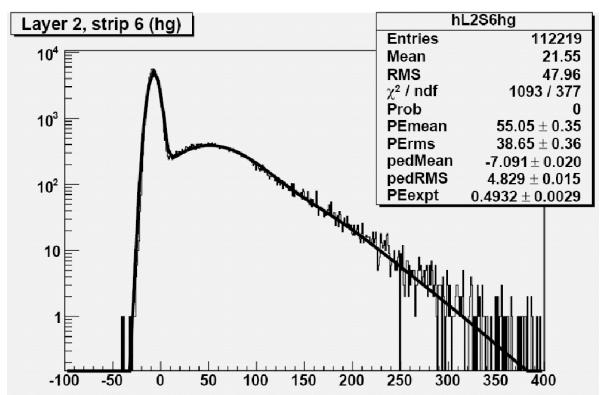

Figure B.8: An example of fitting a p.e. distribution. The x-axis is in units of ADC counts. The highest peak is the pedestal. The second highest peak is the single p.e. peak. Subsequent peaks cannot be distinguished from each other since they overlap. The $\chi^{2}$ in the plot table is not meaningful because of the fit method used. The pedestal appears negative because the FEBs were AC-coupled. 


\section{B.2.6 Low Gain ADC to High Gain ADC Ratio}

Saturation at large high gain ADC counts occurred in our cosmic ray muon runs. This happens because the TriP chip in the VST electronics begins to saturate at a certain amount of fC. After this value, high gain ADC counts no longer scale linearly with charge and the response becomes approximately logarithmic. We used the low gain ADC after this point since it has a linear range over a much larger range of charge. We found that muons did not saturate the low gain ADC. The following studies use a TriP chip gain setting of 11. At the highest TriP chip gain setting, even low gain ADC counts saturate from a MIP. At the lowest ADC gain setting, we would not be sensitive to MIPs.

We plotted high gain ADC counts versus low gain ADC counts to determine the appropriate ranges for the two gains. We used cosmic ray muon data taken in Ped-Mode for all channels in the VST. By looking at the plots (see Figure B.9), we concluded high gain ADC counts had a linear response from 100 to $700 \mathrm{ADC}$ counts. We then performed a linear fit in ROOT and used the inverse of the slope from that fit as the low gain to high gain ratio. The ratio was used to map low gain ADC counts to high gain ADC counts for channels with high gain ADC counts above the 700 ADC cut-off. Table B.7 gives the ratio for each channel in the VST.

We noticed an under-populated region between 700 and 750 ADC counts in the high gain versus low gain ADC plots. All channels had this behavior although the exact point that it appears varies. This is visible in the scatter plot in Figure B.9. We do not know why this region has so few events and never diagnosed this behavior.

The full procedure for how high and low ADC counts were used went as follows. First, we subtracted pedestals for all VST channels. Second, we looked to see if high gain ADC for a channel was greater than three times the pedestal RMS and 


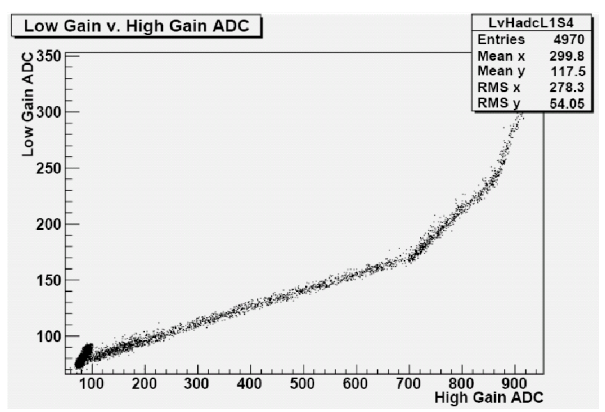

Figure B.9: A scatter plot of high gain ADC versus low gain ADC for VST cosmic ray muons. Nonlinearity begins around 700 high gain ADC counts for this particular channel.

\begin{tabular}{l|c|c|c|c|c|c|c}
\hline \hline & Strip 1 & Strip 2 & Strip 3 & Strip 4 & Strip 5 & Strip 6 & Strip 7 \\
\hline \hline Layer 1 & 0.143 & 0.141 & 0.139 & 0.143 & 0.147 & 0.140 & 0.143 \\
\hline Layer 2 & 0.132 & 0.140 & 0.148 & 0.147 & 0.137 & 0.147 & 0.144 \\
\hline Layer 3 & 0.135 & 0.149 & 0.149 & 0.142 & 0.137 & 0.142 & 0.152 \\
\hline
\end{tabular}

Table B.7: Low gain over high gain ADC ratios for each VST strip.

less than $700 \mathrm{ADC}$ counts. If high gain $\mathrm{ADC}$ was greater than $700 \mathrm{ADC}$ counts, we took the low gain ADC and scaled it to high gain ADC counts using the low gain to high gain ratio. Third, we took high gain ADC, or scaled ADC in the case low gain ADC was needed, and converted that into p.e.

\section{B.2.7 VST Tracking}

We performed tracking to properly identify single cosmic ray muons. In the first step of our tracking, we constructed a cluster for each layer. For the VST, a cluster can be made up of either one or two strips in a layer. We constructed clusters by first finding the strip in each layer with the highest number of p.e. We called this the seed strip. To qualify as a seed strip, a strip cannot be on the edge of the array and the strip must have a signal greater than three times the pedestal RMS above the pedestal. This cut off was usually about 0.3 p.e., but varied slightly channel to 


\begin{tabular}{l|c|c|c|c|c|c|c}
\hline \hline & Strip 1 & Strip 2 & Strip 3 & Strip 4 & Strip 5 & Strip 6 & Strip 7 \\
\hline \hline Layer 1 & 0.0 & 9.6 & 17.2 & 0.0 & 0.0 & 0.0 & 0.0 \\
\hline Layer 2 & 0.0 & 6.5 & 5.2 & 0.1 & 0.0 & 0.0 & 0.0 \\
\hline Layer 3 & 0.9 & 13.6 & 2.9 & 0.0 & 3.8 & 16.3 & 0.2 \\
\hline
\end{tabular}

Table B.8: An example of a pathological event in the VST. Values are in units of p.e. Notice the p.e. deposit on strips 2 and 3 of each layer consistent with a muon. Then note the spurious p.e. deposit on Strip 6, Layer 3.

channel.

In the second step of tracking, we looked to see if strips adjacent to the seed strip qualify as shoulder strips. To qualify as a shoulder strip, the strip needed to make the same minimum cut mentioned for seed strips and must have the higher p.e. count out of the two strips adjacent to the seed strip. We repeated this procedure to find a seed and shoulder strip for each layer.

We experimented with several cuts to screen for valid single muon events. The cut we found to work best was

$$
\left(\mathrm{L}_{\text {p.e. }}-\mathrm{C}_{\text {p.e. }}\right)<3 \text { p.e., }
$$

where $L_{\text {p.e. }}$ is the sum of all p.e. on all strips in a layer and $C_{\text {p.e. }}$ is the sum of p.e. deposited on strips within a cluster. We were motivated by events that had three or four strips within a layer with very large p.e. deposits. Since we are interested in the position resolution of muons and it is not clear that these events are muons, we cut out such events.

An anecdotal reason for this cut is shown in Table B.8. We justify excluding such events because the algorithm we used for finding muons is rather simplistic. We wanted to test the resolution of the array and due to reasonable statistics, did not need to worry about the efficiency of the muon tagging. 
We also used timing information in tracking. We only implemented tracking with timing for runs in Discriminator-Mode. For obvious reasons, we did not use timing information in Ped-Mode. The details of using timing for tracking can be found in Section B.3.4.

\section{B.3 The Results of the VST}

We used the VST to establish whether the scintillator, fiber, PMT, and electronics selected for MINER $\nu$ A were sufficient for meeting the physics goals for the experiment. Below, we describe the various measurements we made.

\section{B.3.1 Light Yield}

We determined the light yield for runs in both Ped-Mode and Discriminator Mode. Only events that passed our single muon cut were included in the light yield study. Not using this cut would generate an inflated light yield value.

We used different procedures to find layer light yield for data taken in Ped-Mode and Discriminator Mode. For Ped-Mode, we summed the p.e. deposit on each strip within a layer. For Discriminator mode, we summed p.e. from each channel with a discriminator that fired in the sum for the layer. A plot of light yield for a layer taken in Ped-Mode is shown in Figure B.10.

Next, we calculated the expected energy deposit for a muon going through a layer. The expected energy deposit is given by

$$
\mathrm{E}_{\text {deposit }}=\rho * l *\left(\frac{\mathrm{dE}}{\mathrm{dx}}\right)_{\min },
$$




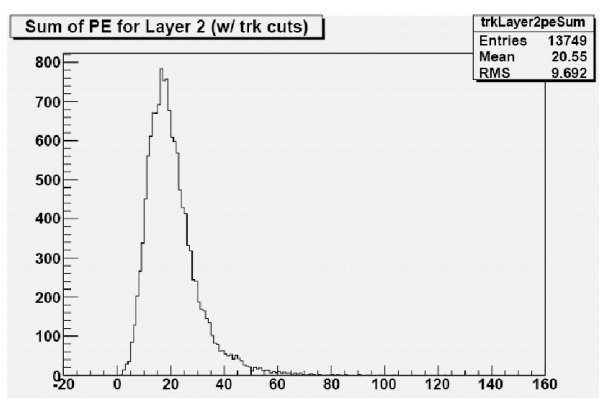

Figure B.10: The light yield for layer 2 in terms of p.e. with data taken in Ped-Mode.

where for polystyrene scintillator, $\left(\frac{\mathrm{dE}}{\mathrm{dx}}\right)_{\min }=1.936$ and the density $\rho=1.032 \frac{\mathrm{g}}{\mathrm{cm}^{3}}[5]$. For the VST array, we have a path length of $l=1.6 \mathrm{~cm}$. The above values give $\mathrm{E}_{\text {deposit }}=3.3 \mathrm{MeV}$. To find the light yield in units of $\frac{\mathrm{pe}}{\mathrm{MeV}}$ for each layer, we divided the p.e. sum for each layer by $\mathrm{E}_{\text {deposit }}$.

This result needed to be scaled to the expected light in the MINER $\nu \mathrm{A}$ detector. We project the MINER $\nu$ A light levels to be 1.093 times the VST result Section B.1.7. After the scaling, we found a value of $6.3 \frac{\mathrm{pe}}{\mathrm{MeV}}$ in Ped-Mode and $6.5 \frac{\mathrm{pe}}{\mathrm{MeV}}$ in Discriminator Mode. The minimum light spec for MINER $\nu \mathrm{A}$ is $4 \frac{\mathrm{pe}}{\mathrm{MeV}}$. Table B.9 shows light yield results for data taken in Ped-Mode.

\section{B.3.2 Position Residual}

Once we completed our tracking and light yield measurements, we looked at the position resolution of the VST array. We assumed that the center of each strip in a layer was separated by $17 \mathrm{~mm}$ from its nearest neighbor and that all three layers were directly over each other.

We found the position resolution by first finding a p.e. weighted position for a cluster. We did this calculation in the same fashion as a center of mass calculation. We weighted the strip position with that strips number of p.e. and summed over 


\begin{tabular}{c|c|c}
\hline \hline Layer & Mean p.e. & $\frac{\text { p.e. }}{\text { MeV }}$ \\
\hline \hline 1 & 20.4 & 6.2 \\
\hline 2 & 20.6 & 6.2 \\
\hline 3 & 21.1 & 6.4 \\
\hline \hline Avg & 20.7 & 6.3 \\
\hline \hline
\end{tabular}

Table B.9: The mean number of layer pe and $\frac{\text { p.e. }}{\mathrm{MeV}}$ for each layer. These results are for Ped-Mode.

both strips in the cluster in the case we had a two strip cluster. We then divided through by the total number of p.e. for that cluster.

Once we found the p.e. weighted position for each cluster, we made a projected position. We found this projected position by averaging the muon positions in layer 1 and layer 3. Since the muons are downward going and their motion nearly perpendicular to the array, this projected position should be close to the position found in layer 2. The position residual is the projected position of the muon minus the measured position in layer 2. A plot of position resolution is shown in Figure B.11. Equation B.6 shows the relationship between the RMS of the position residual and position resolution. The $\sqrt{\frac{3}{2}}$ term comes from statistics.

$$
\mathrm{R}_{\text {position }}=\sqrt{\frac{3}{2}} \mathrm{RMS}_{\text {residual }},
$$

One check we made regarding our position residual was to plot position residual versus the position found in Layer 2. A plot of this can be seen in Figure B.12. We were quite surprised to find an oscillatory behavior. We speculated several reasons why this behavior might exist.

One idea we had was that the layers might not be aligned well. This poor 


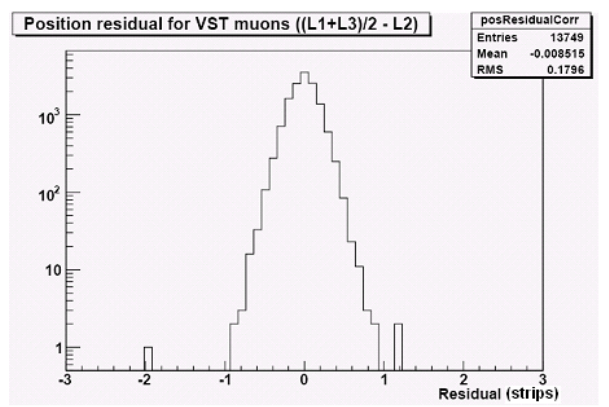

Figure B.11: A log plot of position residual for a run taken in Ped-Mode. The distribution is well peaked with only a few outlying events. The distance between strips on the $\mathrm{x}$-axis is $17 \mathrm{~mm}$.

alignment theory was discounted after inserting the actual position of each strip. Initially, we had assumed a separation between each strip of $17 \mathrm{~mm}$. Before installation, we carefully measured the array to the nearest tenth of a millimeter. We found including the exact position of all strips improved resolution by about $0.1 \%$. We interpreted this result to mean that our strips were very precisely positioned, almost exactly $17 \mathrm{~mm}$ apart, and that each of the three layers was almost exactly overtop one another.

Another idea was that a small unaccounted for strip gap between strips may be causing this behavior. An illustration of this strip gap can be seen in Figure B.13. We have two ideas of what may cause this strip gap. The first is that there may be a small gap between the strips. The other cause could be the coextrusion. The coextrusion has a finite thickness and in addition, scintillator near that extrusion may have a lower response thus giving an effective strip gap. Whatever the cause, we implemented a strip gap correction in the hopes that this would improve our resolution and remove the oscillatory behavior in Figure B.12. We derived a correction term, as seen in Equation B.7 below, accounting for a small gap between strips. 


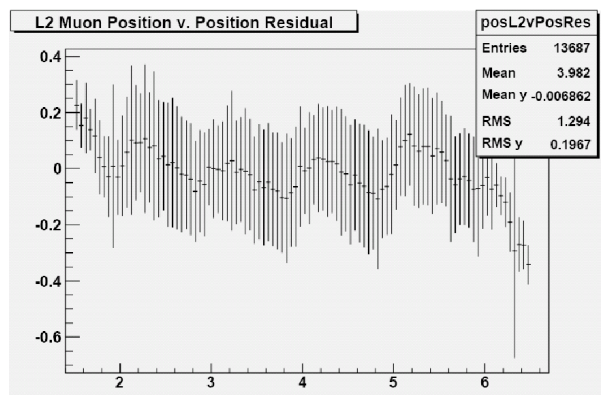

Figure B.12: A plot of muon position in layer 2 versus position residual for that event. The $\mathrm{x}$-axis is in units of strips which are $17 \mathrm{~mm}$ apart. Position residual was found to oscillate depending upon muon position. That lead us to consider a strip gap correction.

$$
\mathrm{C}_{\text {stripgap }}=\frac{\mathrm{L}_{\mathrm{n}-1}-\mathrm{L}_{\mathrm{n}}}{\mathrm{L}_{\mathrm{n}-1}+\mathrm{L}_{\mathrm{n}}} *(\delta-\mathrm{w}),
$$

The $\mathrm{L}_{\mathrm{n}-1}$ and $\mathrm{L}_{\mathrm{n}}$ terms represent the number of p.e. on the $\mathrm{n}-1^{\text {th }}$ and $\mathrm{n}^{\text {th }}$ strip, respectively. The term $\delta$ is the distance between the midpoints of two strips. The value $\mathrm{w}$ is the distance between the center of a strip and the edge of the active region of the strip. For the VST, $\delta$ and w were not equal. The same will likely be true of MINER $\nu$ A. The term $(\delta-\mathrm{w})$ is what we called the strip gap. This whole strip gap correction got added onto the p.e. weighted postion. We experimented with different strip gaps and found that $0.7 \mathrm{~mm}$ gave the optimum improvement in the strip gap correction. The effect of this correction amounts to a roughly $1 \%$ improvement in resolution. Although the strip gap correction improved the position resolution, it did not eliminate the oscillatory behavior that initially motivated this study. Including our cut, correction terms, and using data taken in Discriminator-Mode, we found a position resolution of $2.5 \mathrm{~mm}$. 


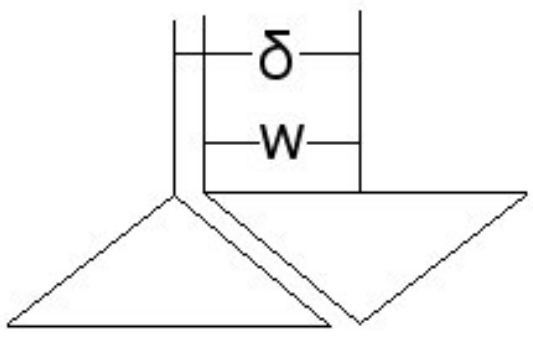

Figure B.13: An illustration of the difference between strip width and strip separation. The difference $\delta$ - w we refer to as the strip gap.

\begin{tabular}{c|c|c|c|c}
\hline \hline Layer & 0.5 & 0.63 & 0.8 & No Filter \\
\hline \hline 1 & 8.7 & 11.8 & 15.3 & 20.4 \\
\hline 2 & 9.2 & 12.4 & 15.8 & 20.6 \\
\hline 3 & 8.9 & 12.1 & 15.6 & 21.1 \\
\hline \hline Avg & 8.9 & 12.1 & 15.6 & 20.7 \\
\hline \hline Actual Transmission & 0.43 & 0.58 & 0.75 & 1.0 \\
\hline \hline
\end{tabular}

Table B.10: Light yields (p.e.) for different filters and the no filter case. Dividing light yield by the no filter case gives the actual light transmission for a particular filter.

\begin{tabular}{c|c|c}
\hline \hline Filter & Effective Transmission & Resolution $(\mathrm{mm})$ \\
\hline \hline 0.5 & 0.43 & 3.7 \\
\hline 0.63 & 0.59 & 3.2 \\
\hline 0.8 & 0.75 & 2.8 \\
\hline 1.0 & 1.0 & 2.5 \\
\hline \hline
\end{tabular}

Table B.11: Position resolutions for different light yields.

\section{B.3.3 The Effect of Reduced Light Levels on Position Res- olution}

The position resolution we found in Section B.3.2 matched our expectations; however, we examined how the position resolution scales with lower light yields to understand how reduced light would impact MINER $\nu$ As physics reach. This is useful for identifying the minimum acceptable light yield. 


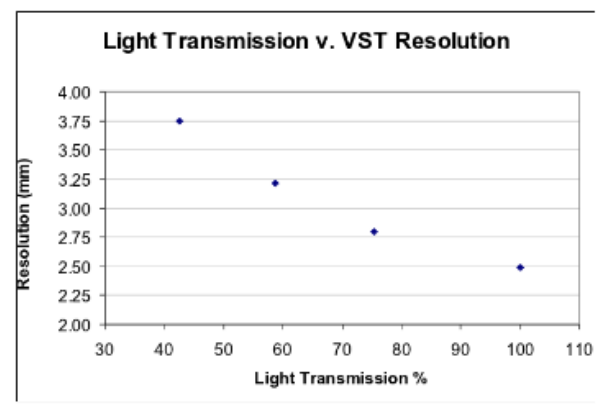

Figure B.14: A plot showing the improvement of position resolution with increasing amounts of light yield. Light yield of $100 \%$ corresponds to $6.3 \frac{\mathrm{pe}}{\mathrm{MeV}}$. Position resolution rapidly deteriorates as light yield is reduced.

To perform these studies, we used Kodak Wratten Neutral Density Filters [138]. These filters are about 4 mils thick and can be cut easily with scissors. We trimmed them to match the size and shape of the MINOS fiber connectors. Next, we inserted them between the MINOS fiber connectors and the CALDET box. Filters were held in place by friction. We did a run for each of the three filters in place and one run without a filter. The advertised light transmission for each filter was slightly higher than the effective transmission. Actual light transmission values are listed in Table B.11. All runs, with and without a filters, were taken in Ped-Mode.

We found that position resolution drops off rapidly with decreasing light yield as is illustrated in Figure B.14. Results are summarized in Table B.11. Fortunately, we expect the actual light yield in MINER $\nu \mathrm{A}$ to be slightly higher than what we had in the VST. This means position resolution should be comparable or slightly higher than in the VST.

\section{B.3.4 Timing Resolution}

To find the timing resolution, we had to tune the discriminators on each FEB individually. There is an inverse relationship between the VTH setting and the ADC 


\begin{tabular}{c|c}
\hline \hline FEB & VTH Setting \\
\hline \hline 5 & 227 \\
\hline 6 & 225 \\
\hline 7 & 228 \\
\hline 8 & 232 \\
\hline \hline
\end{tabular}

Table B.12: Optimum VTH settings for each VST FEB.

threshold. The higher the VTH bit setting, the lower the ADC threshold will be. VTH bit settings varied from 0 to 255 .

First, we found the VTH value where the discriminator fires on the pedestal. From that setting, we then increased the ADC threshold by decreasing the VTH bit setting. We raised this bit setting until the discriminator no longer fired on pedestal. We viewed the bit setting where this first happens as the ideal VTH setting. The VTH settings are shown in Table B.12. The goal of this tuning was to have sensitivity to single p.e.'s while not being susceptible to triggering on noise.

With FEB thresholds tuned, we took data with cosmic ray muons in Discriminator Mode. The timing for a channel is given by adding the value found on the discriminator of a channel to the FEB clock. We then multiplied through by $9.8 \mathrm{~ns}$, the length of a clock tick. We found large timing difference between channels, much greater than can be accounted for by time of flight.

We applied a calibration for FEB clock times to remove these timing differences. The clocks on all four FEBs should be synchronized to within 1 ns of each other. A muon takes $0.6 \mathrm{~ns}$ to pass through a single layer. Accounting for the small gaps between layers, that means a muon takes about 2 ns to pass through the entire array. That would lead one to naïvely guess that shoulder and seed strips in a layer will have a negligible time difference and that time differences between strips for an event should be about 2 ns. 
We found a difference much greater than the 1-2 ns originally anticipated. Differences ranged from -2 ns to $10 \mathrm{~ns}$, yet all had a very well peaked distribution. This is consistent with FEBs clocks being slightly unsynchronized. We measured an offset term for each FEB and included it to remove large timing differences.

To incorporate the timing information into our tracking we first calculated the charge weighted time average for each event. We first multiplied the number of p.e. on a channel by the clock value for that channel. This was done for any VST channel in an event with a discriminator that fired. We summed all such products in an event and divided through by the sum of all charge for that event. We then reiterated this calculation excluding any events that were more than $100 \mathrm{~ns}$ away from our charge weighted time average. We used this method since one channel can have up to four hits on it within a gate. We then made a tracking requirement that any seed or shoulder strips in our tracking had to be within 100 ns of the newly calculated charge weighted time average.

To find the timing resolution, we took timing differences between seed strips of adjacent layers. We required seed strips to be directly overtop one another. For example, we used timing differences like $\left(\mathrm{Clk}_{\mathrm{L} 2 \mathrm{~S} 2}-\mathrm{CLk}_{\mathrm{L} 1 \mathrm{~S} 2}\right)$ or $\left(\mathrm{Clk}_{\mathrm{L} 3 \mathrm{~S} 3}-\mathrm{Clk}_{\mathrm{L} 2 \mathrm{~S} 3}\right)$, but not $\left(\mathrm{Clk}_{\mathrm{L} 3 \mathrm{~S} 2}-\mathrm{Clk}_{\mathrm{L} 1 \mathrm{~S} 2}\right)$. To get the timing resolution for a particular channel, we divided the RMS of the timing difference by $\sqrt{2}$, which comes from statistics. An example of a timing difference for a channel is shown in Figure B.15. We averaged individual resolutions for each VST channel to get the timing resolution. Our timing resolution was $3.4 \mathrm{~ns}$. The granularity of our timing resolution is $2.4 \mathrm{~ns}$. Timing resolution should be close to the granularity, but we find a result slightly worse than that.

We found an even more substantial spread in the RMS when we looked at the 


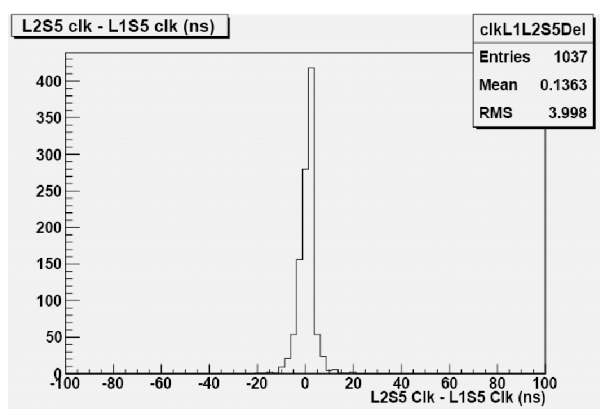

Figure B.15: A plot of the timing difference between two seed strips. The difference is narrowly peaked close to zero after calibrating for FEB timing misalignments.
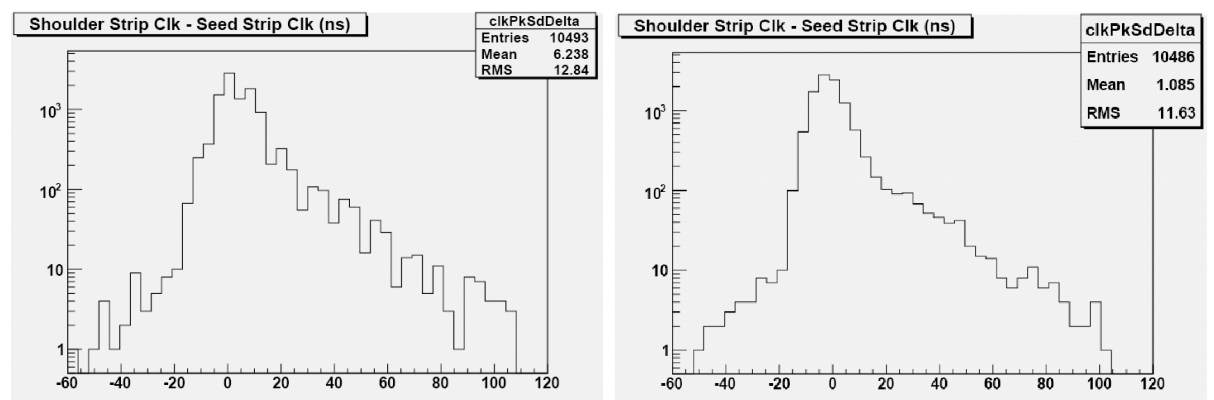

Figure B.16: On the left is a plot of the timing difference between shoulder and seed strips (in nanoseconds) without the charge slewing correction term. On the right is a plot of the same quantity, but with that correction term. In both cases, the distribution is substantially broader than the timing differences between seed strips.

timing difference between a seed strip and a shoulder strip within a cluster (see Figure B.16). We conjectured that the large difference in timing resulted from charge slewing. Charge slewing happens because of leading edge effects in signals. The discriminator fires when a set amount of charge is integrated. This integration takes longer for a small signal compared to a large signal. In other words, large signals have a steeper rising edge and can pass threshold first creating the appearance that the event had a major timing difference between strips.

To compensate for charge slewing, we introduced a slewing correction

$$
\text { SlewingCorrection }=15 *\left(\left(\mathrm{pe}_{\text {seed }}\right)^{0.7}-\left(\mathrm{pe}_{\text {shoulder }}\right)^{0.7}\right) \text {, }
$$


where $\mathrm{pe}_{\text {seed }}$ is the p.e. in the seed strip, pe $\mathrm{s}_{\text {shoulder }}$ is the number of p.e. in the shoulder strip, and we found the constants 15 and 0.7 by tuning parameters. We adopted this arbitrary function solely for its behavior. The function is small if both seed p.e. and shoulder p.e. are either high or low, but large if seed p.e. is high, but shoulder p.e. is small. This gives a small decrease in RMS as seen in Figure B.16. The correction term did not remove the large timing differences.

Overall, we found a timing resolution of 3.4 ns. This is slightly worse than expected, but sufficient for MINER $\nu$ A's physics needs. We speculate that the decay constant of the WLS fiber may be causing additionaly smearing in the timing.

\section{B.3.5 Concluding Thoughts on the VST}

The VST verified that all basic systems of the MINER $\nu$ A detector work, particularly the electronics. We demonstrated that we have a light yield of $6.5 \frac{\mathrm{pe}}{\mathrm{Mev}}$ which is above the minimum specification of $4.0 \frac{\mathrm{pe}}{\mathrm{MeV}}$. We found a position resolution of $2.5 \mathrm{~mm}$ for muons, slightly better than the roughly $3 \mathrm{~mm}$ we expected. Our timing resolution of $3.4 \mathrm{~ns}$ is below our expectation, but is close to the granularity of our timing. 


\title{
Appendix C
}

\section{List of MINER $\nu \mathrm{A}$ Collaborators}

\author{
G. Tzanakos \\ University of Athens \\ J. Cravens, M. Jerkins, S. Kopp, J. Ratchford \\ University of Texas at Austin \\ Palomino \\ Centro Brasileiro de Pesquisas Físicas \\ J. Grange, J. Mousseau, B. Osmanov, H. Ray \\ University of Florida \\ D. W. Schmitz \\ University of Chicago
}

D.A.M. Caicedo, C.M. Castromonte, H. da Motta, G. A. Fiorentini, K. Hurtado, J.L. 
D. Boehnlein, R. DeMaat, L. Gilbert, N. Grossman, D. A. Harris, J. G. Morfín, J. Osta, R. B. Pahlka, E. A. Paschos, P. Rubinov, F.D. Snider, R. Stefanski Fermilab

A. Blondel, A. Bravar, Y. Karadzhov, A. Korzenev, C. Martin Mari University of Geneva

J. Felix, A. Higuera, Z. Urrutia, E. Valencia, G. Zavala Universidad de Guanajuato

M.E. Christy, , C. Keppel, , T. Walton, L. Y. Zhu Hampton University

A. Butkevich, S.A. Kulagin Inst. Nucl. Reas. Moscow

G. Niculescu, I. Niculescu James Madison University

E. Maher

Mass. Col. Lib. Arts

L. Fields, B. Gobbi, Cheryl E. Patrick, L. Patrick, H. Schellman Northwestern University

\author{
N. Tagg \\ Otterbein University
}


S. Boyd, I. Danko, S.A. Dytman, B. Eberly, Z. Isvan, C.L. McGivern, D. Naples, V. Paolone University of Pittsburgh

G. Díaz, A. M. Gago, J.P. Velasquez

Pontificia Universidad Catolica del Peru

\author{
R. Napora \\ Purdue University Calumet
}

S. Avvakumov, A. Bodek, R. Bradford, H. Budd, J. Chvojka, M. Day, H. Lee, L. Loiacono, S. Manly, C. M. Marshall, K.S. McFarland, A. M. McGowan, A. Mislivec, J. Park, G. Perdue, P. A. Rodrigues, J. Wolcott

University of Rochester

G. J. Kumbartzki, T. Le, R. D. Ransome, E. C. Schulte, B. G. Tice

Rutgers University

O. Altinok, H. Gallagher, T. Kafka, W.A. Mann, W. P. Oliver, Tufts University

C. Simon, B.P.Ziemer

University of California at Irvine

R. Gran, M. Lanari

University of Minnesota at Duluth 
M. Alania, C. J. Solano Salinas

Universidad Nacional de Ingeniería

W. K. Brooks, G. Maggi, C. Peña, I.K. Potashnikova, F. Prokoshin Universidad Técnica Federico Santa María

L. Aliaga, J. Devan, M. Kordosky, J.K. Nelson, A. Norrick, J. Walding, D. Zhang College of William and Mary 